\title{
An anti-Carbopalladation/Amination Cascade with Alkynes: Access to Tetrasubstituted Enamines and Pyrroles
}

Theresa Schitter, ${ }^{\dagger}$ Simon Stammwitz, ${ }^{\dagger}$ Peter G. Jones, ${ }^{\dagger}$ and Daniel B. Werz ${ }^{*}, \dagger$

$\dagger$ Technische Universität Braunschweig, Institut für Organische Chemie and $\$$ Technische Universität Braunschweig, Institut für Anorganische und Analytische Chemie, Hagenring 30, 38106 Braunschweig, Germany

\section{Supporting Information - Table of Contents}

1) General Experimental $\quad \mathrm{S} 2$

2) General procedures (GP) S3

3) Syntheses of Precursor of Type $\mathbf{1}$ and Domino Products of Type $3 \quad$ S5

3a) Syntheses of Tosylates $\quad$ S6

3b) Syntheses of Precursors of Type 1

3c) Syntheses of Domino Products of Type $3 \quad$ S24

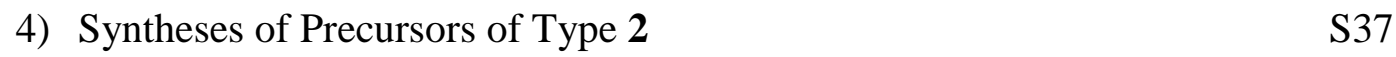

5) Syntheses of Domino Products of Type $4 \quad$ S58

6) Syntheses of Domino Products of Type $5 \quad$ S65

7) Termination with different Amines $\quad$ S84

$\begin{array}{ll}\text { 8) Follow-up chemistry } & \text { S91 }\end{array}$

9) Crystal Structure Determination of Compound 3f $\quad$ S104

$\begin{array}{lr}10) \text { References } & \text { S106 }\end{array}$ 


\section{1) General Experimental}

All solvents were dried and stored over molecular sieves under Argon atmosphere unless otherwise stated. Air- and moisture-sensitive reactions were carried out in oven-dried or flame-dried glassware, septum-capped under atmospheric pressure of argon. Commercially available compounds were used without further purification unless otherwise stated.

Proton $\left({ }^{1} \mathrm{H}\right)$, carbon $\left({ }^{13} \mathrm{C}\right)$ and fluorine $\left({ }^{19} \mathrm{~F}\right)$ NMR spectra were recorded on a Bruker AV300, Bruker AVIII400, Bruker AVIIIHD500 or Bruker AVII600 instrument using the residual signals from $\mathrm{CHCl}_{3}, \delta=7.26 \mathrm{ppm}$ and $\delta=77.16 \mathrm{ppm}$, as internal reference for ${ }^{1} \mathrm{H}$ and ${ }^{13} \mathrm{C}$ chemical shifts, respectively. Additionally, tetramethylsilane (TMS; $\delta=0.00 \mathrm{ppm} ; 0.03 \%$ ) was added to NMR samples. The following abbreviations were used for NMR chemical shifts: $\mathrm{s}=$ singlet, $\mathrm{d}=$ doublet, $\mathrm{t}=$ triplet, $\mathrm{q}=$ quartet, $\mathrm{m}=$ multiplet and combinations thereof. ESI-HRMS mass spectrometry was carried out on an FTICR instrument. GC-HRMS mass spectrometry was carried out on an Agilent 6890 gas chromatograph coupled to a JMST100GC (GCAccuTOF, JEOL, Japan) time of flight mass spectrometer in electron ionization (EI) mode. IR spectra were recorded on an ATR spectrometer Tensor 27 from Bruker. Melting Points were recorded with a Büchi SMP-20 melting point meter and are uncorrected. 


\section{2) General procedures (GP)}

\section{GP1: Synthesis of Tosylate}

To a solution of alcohol (1.0 equiv) in $\mathrm{CH}_{2} \mathrm{Cl}_{2}(10.0 \mathrm{~mL} / \mathrm{mmol})$ was added triethylamine (1.4-3.0 equiv) and 4-dimethylaminopyridine (catalytic amount) at $0{ }^{\circ} \mathrm{C}$. Afterwards tosyl chloride (1.1-1.6 equiv) was added in several portions. The mixture was stirred overnight at ambient temperature. To the reaction mixture was added $\mathrm{NaOH}$-solution (2M) and the layers were separated. The aqueous layer was extracted with $\mathrm{CH}_{2} \mathrm{Cl}_{2}(3 \times)$. The combined organic layers were washed with brine, dried over $\mathrm{Na}_{2} \mathrm{SO}_{4}$, and concentrated in vacuo. The residue was purified by silica gel column chromatography.

\section{GP2: Synthesis of Amine (A)}

The tosylate (1.0 equiv) was dissolved in DMF ( $4 \mathrm{~mL} / \mathrm{mmol})$. To the solution were added potassium iodide (catalytic amount), amine (1.4-2.5 equiv) and potassium carbonate (2.8-3.2 equiv) and the mixture was stirred at $90{ }^{\circ} \mathrm{C}$ (oil bath) until TLC showed full conversion of the starting material. To the reaction mixture was added $\mathrm{NH}_{4} \mathrm{Cl}$-solution and the layers were separated. The aqueous layer was extracted with EtOAc (3x). The combined organic layers were washed with brine, dried over $\mathrm{Na}_{2} \mathrm{SO}_{4}$, and concentrated in vacuo. The residue was purified by Silica gel column chromatography.

\section{GP3: Pd-catalyzed Domino Reaction}

The domino precursor (1.0 equiv) was dissolved in DMF $(3.0 \mathrm{~mL} / 100 \mu \mathrm{mol})$. The solution was degassed with argon for $10 \mathrm{~min}$. Afterwards base (5.0 equiv), ligand (10 mol \%) and Pdcatalyst $(5 \mathrm{~mol} \%)$ were added in that order. The mixture was stirred $30 \mathrm{~min}$ at $\mathrm{rt}$ and afterwards at $120{ }^{\circ} \mathrm{C}$ (oil path) until TLC showed full conversion of the starting material. After cooling to rt, water was added to the reaction mixture. The layers were separated and the aqueous layer was extracted with EtOAc $(3 \times)$. The combined organic layers were washed with brine, dried over $\mathrm{Na}_{2} \mathrm{SO}_{4}$, and concentrated in vacuo. The residue was purified by silica gel column chromatography.

\section{GP4: Synthesis of Amine (B)}

The alcohol (1.0 equiv) was dissolved in $\mathrm{CH}_{2} \mathrm{Cl}_{2}(10 \mathrm{~mL} / \mathrm{mmol})$. To the solution were added $\mathrm{N}, \mathrm{N}$-diisopropylethylamine (1.3-2.0 equiv) and triflic anhydride (1.1-1.7 equiv) at $0{ }^{\circ} \mathrm{C}$. The reaction mixture was stirred $30 \mathrm{~min}$ at $0{ }^{\circ} \mathrm{C}$. Afterwards the amine (1.7-2.9 equiv) was added. 
The reaction mixture was stirred at $\mathrm{rt}$ until TLC showed full conversion of the starting material. To the reaction mixture was added $\mathrm{NH}_{4} \mathrm{Cl}$-solution and the layers were separated. The aqueous layer was extracted with EtOAc $(3 \times)$. The combined organic layers were washed with brine, dried over $\mathrm{Na}_{2} \mathrm{SO}_{4}$, and concentrated in vacuo. The residue was purified by Silica gel column chromatography. 
3) Syntheses of Precursors of Type 1 and Domino Products of Type 3

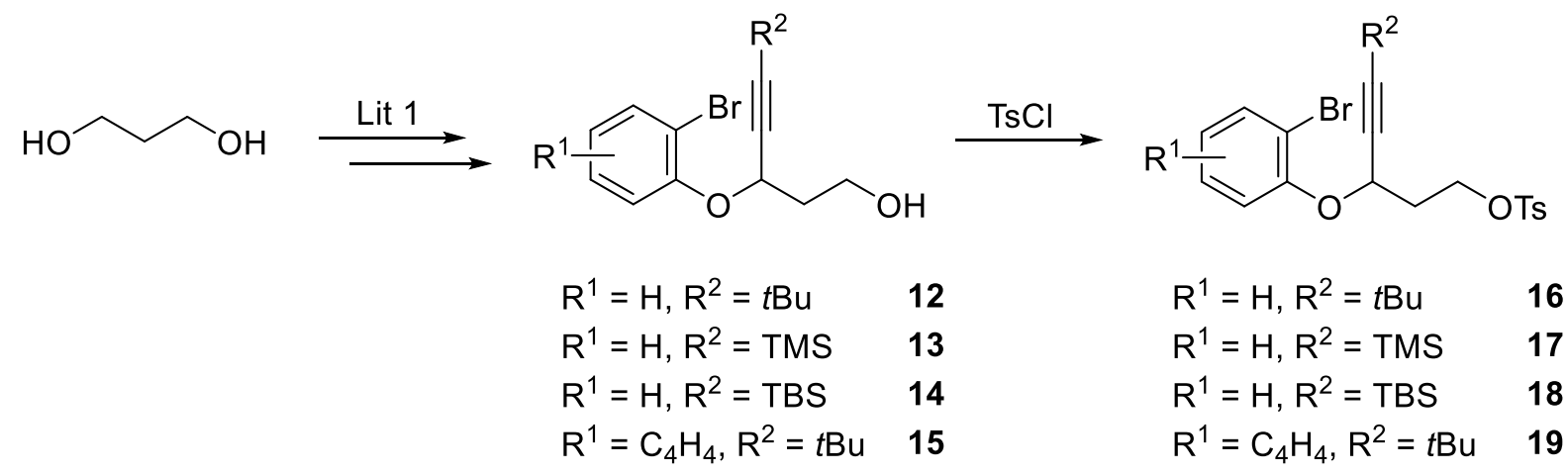

Aniline<smiles>[R]CC#CC(CCNc1ccccc1)Oc1cc[Y]([R])cc1Br</smiles>

$\mathrm{R}^{1}=\mathrm{H}, \mathrm{R}^{2}=t \mathrm{Bu}, \mathrm{R}=\mathrm{H} \quad$ 1a $(73 \%)$

$\mathrm{R}^{1}=\mathrm{H}, \mathrm{R}^{2}=\mathrm{H}, \mathrm{R}=\mathrm{H} \quad 20(46 \%)$

$\mathrm{R}^{1}=\mathrm{H}, \mathrm{R}^{2}=\mathrm{TBS}, \mathrm{R}=\mathrm{H} \quad \mathbf{1 b}(62 \%)$

$\mathrm{R}^{1}=\mathrm{H}, \mathrm{R}^{2}=t \mathrm{Bu}, \mathrm{R}=3,5-(\mathrm{OMe})_{2} \quad 1 \mathrm{c}(42 \%)$

$\mathrm{R}^{1}=\mathrm{H}, \mathrm{R}^{2}=t \mathrm{Bu}, \mathrm{R}=4-\mathrm{Cl} \quad$ 1d $(23 \%)$

$\mathrm{R}^{1}=\mathrm{H}, \mathrm{R}^{2}=\mathrm{TBS}, \mathrm{R}=4-\mathrm{F} \quad$ 1e $(28 \%)$

$R^{1}=$ naphthyl, $R^{2}=t B u, R=H \quad 1 f(20 \%)$
$\mathrm{Pd}(\mathrm{dba})_{2}$

$\left[t \mathrm{Bu}_{3} \mathrm{PH}\right]\left[\mathrm{BF}_{4}\right]$ $\mathrm{K}_{3} \mathrm{PO}_{4}$

DMF, $120^{\circ} \mathrm{C}$

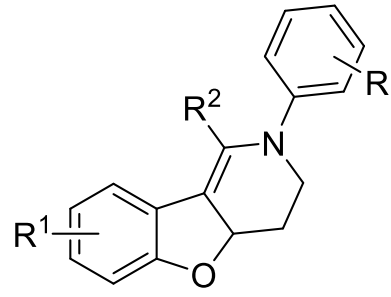

$$
\begin{aligned}
& R^{1}=H, R^{2}=t B u, X=O, R=H \\
& R^{1}=H, R^{2}=T B S, X=O, R=H \\
& R^{1}=H, R^{2}=t B u, X=O, R=3,5-(O M e)_{2} \\
& R^{1}=H, R^{2}=t B u, X=O, R=4-C l \\
& R^{1}=H, R^{2}=T B S, X=O, R=4-F \\
& R^{1}=\text { naphthyl, } R^{2}=t B u, X=O, R=H
\end{aligned}
$$

$3 f(68 \%)$ 


\section{3a) Syntheses of Tosylates}

\section{3-(2-Bromophenoxy)-6,6-dimethylhept-4-yn-1-yl 4-methylbenzenesulfonate (16)}<smiles>CC(C)(C)C#CCCC(O[Sn])Oc1ccccc1Br</smiles>

The alcohol $12^{1}$ (952 mg, $\left.3.06 \mathrm{mmol}\right)$, triethylamine $(580 \mu \mathrm{L}, 423 \mathrm{mg}, 4.18 \mathrm{mmol})$ and tosyl chloride (646 mg, $3.39 \mathrm{mmol}$ ) were reacted according to GP1 (Synthesis of Tosylate). The reaction mixture was stirred $15 \mathrm{~h}$ at rt. After extraction, compound $\mathbf{1 6}(1.42 \mathrm{~g}, 3.05 \mathrm{mmol}$, $100 \%$ ) was obtained as colorless oil.

The product was used without further purification in the next step.

\section{3-(2-Bromophenoxy)-5-(trimethylsilyl)pent-4-yn-1-yl 4-methylbenzenesulfonate (17)}

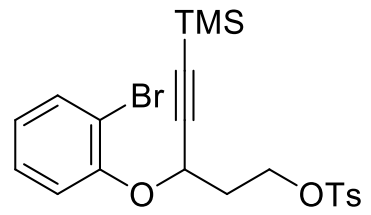

The alcohol $\mathbf{1 3}^{1}$ (2.56 g, $\left.7.82 \mathrm{mmol}\right)$, triethylamine $(2.20 \mathrm{~mL}, 15.9 \mathrm{mmol})$ and tosyl chloride (1.81 g, $9.49 \mathrm{mmol}$ ) were reacted according to GP1 (Synthesis of Tosylate). Silica gel column chromatography (EtOAc: $n$-pentane = 1:20) afforded product $17(3.73 \mathrm{~g}, 7.74 \mathrm{mmol}, 99 \%)$ as colorless oil.

${ }^{1}$ H-NMR (500 MHz, $\left.\mathrm{CDCl}_{3}\right): \delta=0.11(\mathrm{~s}, 9 \mathrm{H}), 2.28-2.36(\mathrm{~m}, 2 \mathrm{H}), 2.37(\mathrm{~s}, 3 \mathrm{H}), 4.28-4.34$ $(\mathrm{m}, 2 \mathrm{H}), 4.74(\mathrm{dd}, J=8.0,5.1 \mathrm{~Hz}, 1 \mathrm{H}), 6.85-6.91(\mathrm{~m}, 1 \mathrm{H}), 7.06(\mathrm{dd}, J=8.3,1.4 \mathrm{~Hz}, 1 \mathrm{H})$, 7.16-7.20 (m, 2H), 7.24 (ddd, $J=8.2,7.3,1.7 \mathrm{~Hz}, 1 \mathrm{H}), 7.48(\mathrm{dd}, J=7.8,1.6 \mathrm{~Hz}, 1 \mathrm{H}), 7.70$ $(\mathrm{d}, J=8.3 \mathrm{~Hz}, 2 \mathrm{H})$.

${ }^{13} \mathrm{C}-\mathrm{NMR}\left(126 \mathrm{MHz}, \mathrm{CDCl}_{3}\right): \delta=-0.4,21.7,35.3,65.6,66.3,93.4,101.4,112.8,115.6$, $122.8,127.8,128.2,129.8,132.4,133.1,144.8,153.6$.

IR (ATR): $\tilde{v}\left(\mathrm{~cm}^{-1}\right)=3064(\mathrm{w}), 2962(\mathrm{w}), 2902(\mathrm{w}), 1474(\mathrm{~m}), 1363(\mathrm{~m}), 1244(\mathrm{~m}), 1180(\mathrm{~s})$. HRMS-ESI: $\mathrm{C}_{21} \mathrm{H}_{25} \mathrm{BrO}_{4} \mathrm{SSi} \mathrm{m} / z$ calcd. $\left[\mathrm{M}+\mathrm{Na}^{+}\right]$: 503.0318 found 503.0320. 


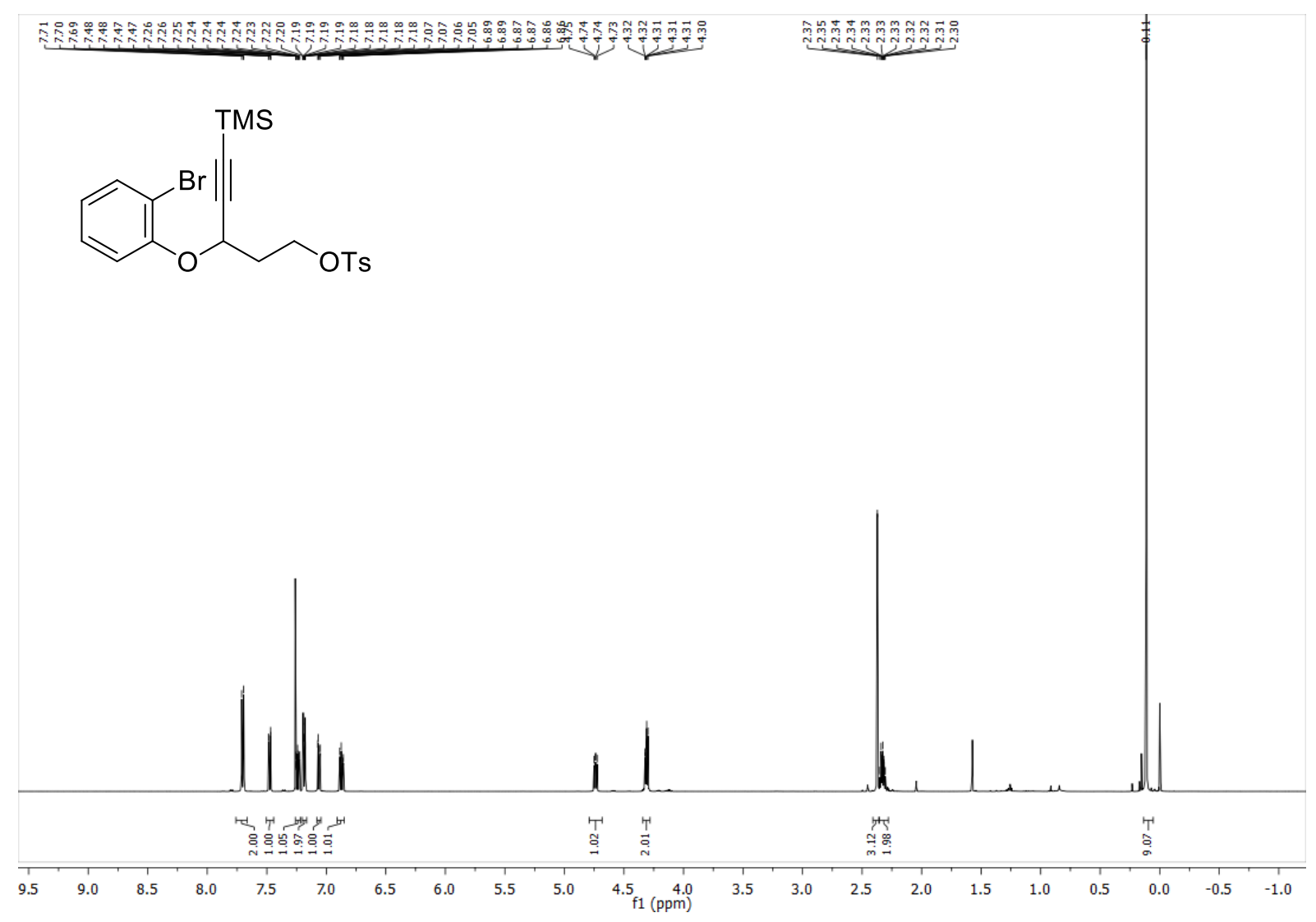

${ }^{1} \mathbf{H}-\mathbf{N M R}\left(500 \mathrm{MHz}, \mathrm{CDCl}_{3}\right)$

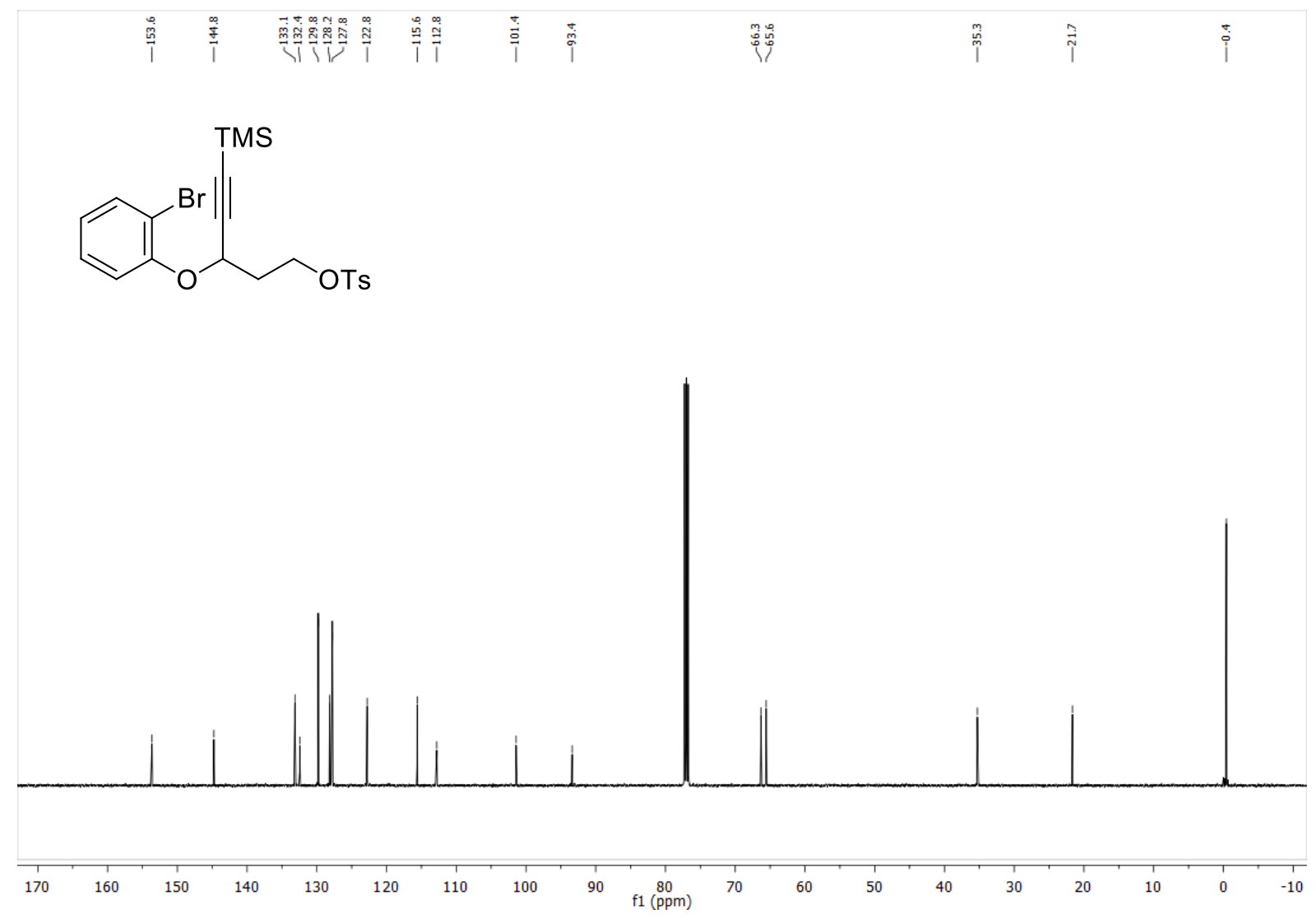

${ }^{13}$ C-NMR $\left(126 \mathrm{MHz}, \mathrm{CDCl}_{3}\right)$ 
<smiles>CC(C)(C)CCCC(C#C[Se-])Oc1ccccc1Br</smiles>

The alcohol $14^{2}(3.81 \mathrm{~g}, 10.3 \mathrm{mmol})$, triethylamine $(4.20 \mathrm{~mL}, 30.3 \mathrm{mmol})$ and tosyl chloride $(2.33 \mathrm{~g}, 12.2 \mathrm{mmol})$ were reacted according to GP1 (Synthesis of Tosylate). The reaction mixture was stirred $14 \mathrm{~h}$ at rt. Silica gel column chromatography (EtOAc: $n$-pentane = 1:20) afforded product $\mathbf{1 8}(5.30 \mathrm{~g}, 10.1 \mathrm{mmol}, 98 \%)$ as colorless oil.

${ }^{1} \mathrm{H}-\mathrm{NMR}\left(300 \mathrm{MHz}, \mathrm{CDCl}_{3}\right): \delta=0.04(\mathrm{~d}, J=1.7 \mathrm{~Hz}, 6 \mathrm{H}), 0.84(\mathrm{~s}, 9 \mathrm{H}), 2.29-2.40(\mathrm{~m}, 5 \mathrm{H})$, 4.26-4.37 (m, 2H), 4.77 (dd, $J=7.4,5.6 \mathrm{~Hz}, 1 \mathrm{H}), 6.86$ (ddd, $J=7.9,7.3,1.5 \mathrm{~Hz}, 1 \mathrm{H}), 7.07$ $(\mathrm{dd}, J=8.3,1.4 \mathrm{~Hz}, 1 \mathrm{H}), 7.15-7.26(\mathrm{~m}, 3 \mathrm{H}), 7.47(\mathrm{dd}, J=7.9,1.6 \mathrm{~Hz}, 1 \mathrm{H}), 7.65-7.75(\mathrm{~m}$, $2 \mathrm{H})$.

${ }^{13}$ C-NMR (76 MHz, $\left.\mathrm{CDCl}_{3}\right): \delta=-4.9,16.4,21.7,25.9,35.3,65.5,66.3,91.8,102.1,112.9$, $115.7,122.8,127.8,128.1,129.8,132.5,133.1,144.8,153.6$.

IR (ATR): $\tilde{v}\left(\mathrm{~cm}^{-1}\right)=2934(\mathrm{~m}), 2892(\mathrm{~m}), 2858(\mathrm{~m}), 1589(\mathrm{w}), 1472(\mathrm{~m}), 1365(\mathrm{~m}), 1244$ (m), $1180(\mathrm{~s})$.

HRMS-ESI: $\mathrm{C}_{24} \mathrm{H}_{31} \mathrm{BrO}_{4} \mathrm{SSi} \mathrm{m} / z$ calcd. $\left[\mathrm{M}+\mathrm{Na}^{+}\right]$: 545.0788 found 545.0792 . 


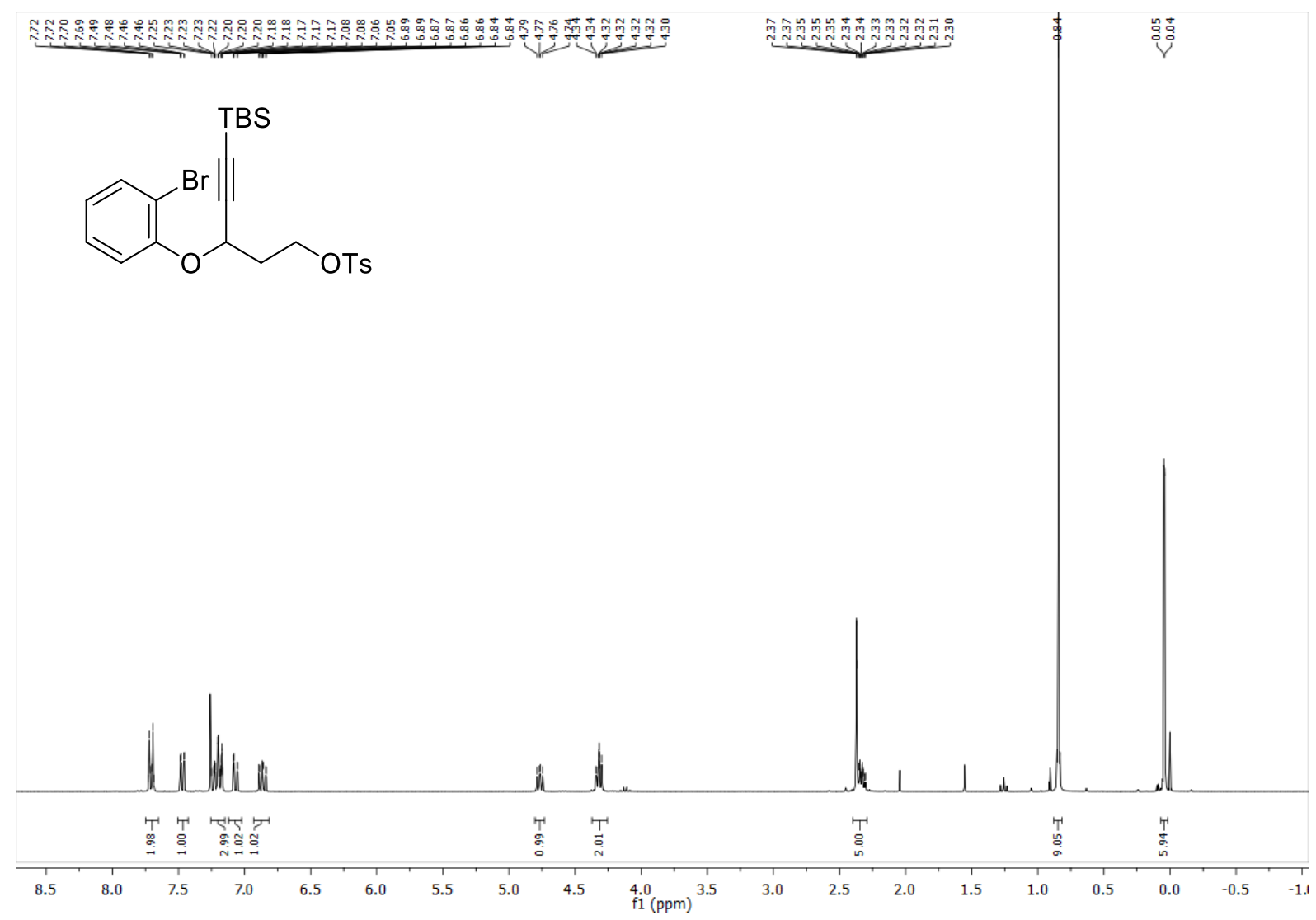

${ }^{1} \mathbf{H}-\mathbf{N M R}\left(300 \mathrm{MHz}, \mathrm{CDCl}_{3}\right)$

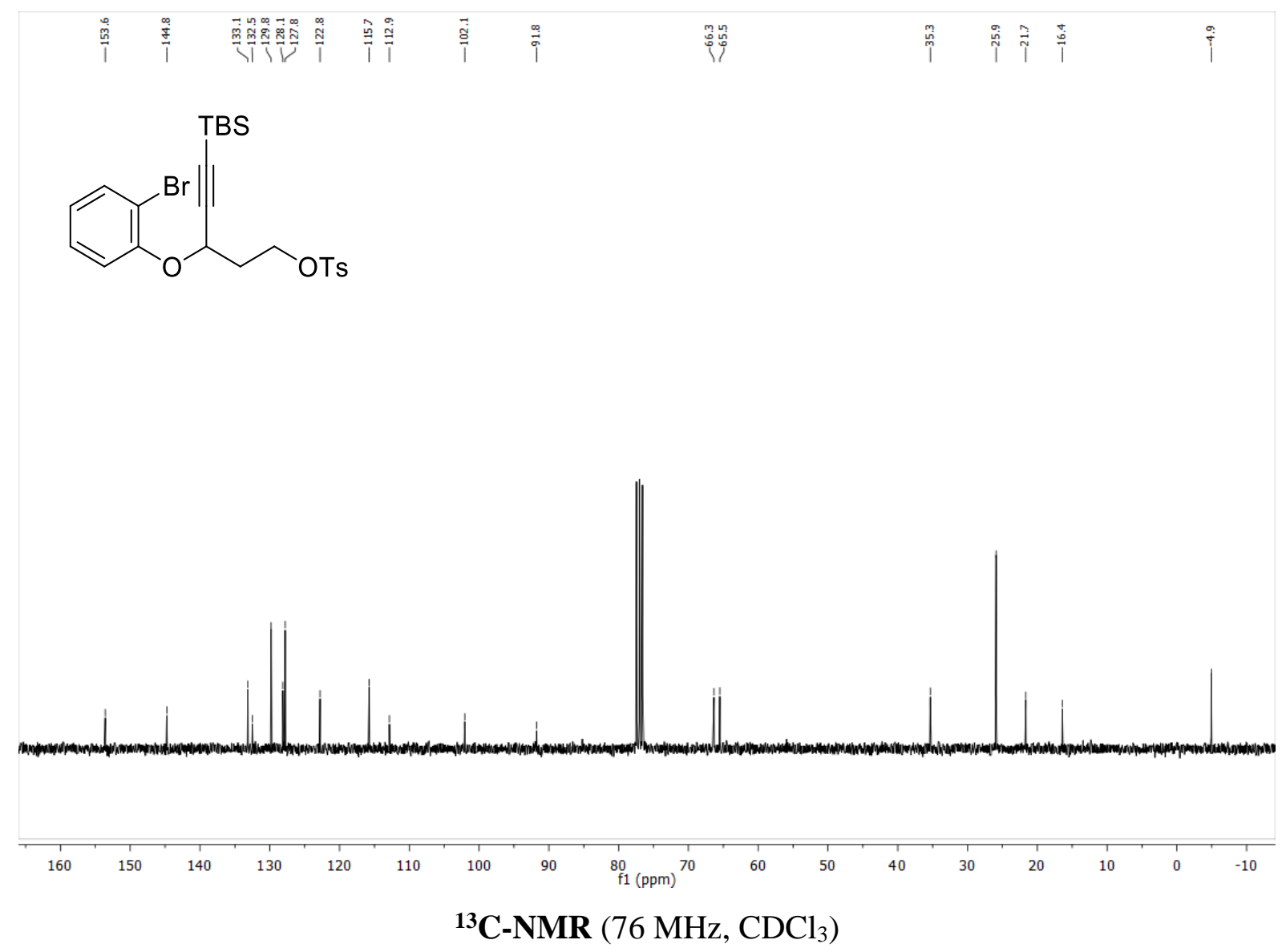




\section{3-((3-Bromonaphthalen-2-yl)oxy)-6,6-dimethylhept-4-yn-1-yl 4-methylbenzenesulfonate}

(19)<smiles>CC(C)(C)C#CC(CC[SeH])Oc1cc2ccccc2cc1Br</smiles>

The alcohol $15^{1}(162 \mathrm{mg}, 447 \mu \mathrm{mol})$, triethylamine $(150 \mu \mathrm{L}, 1.08 \mathrm{mmol})$ and tosyl chloride (135 mg, $708 \mu \mathrm{mol})$ were reacted according to GP1 (Synthesis of Tosylate). The reaction mixture was stirred $15 \mathrm{~h}$ at rt. After extraction compound 19 (195 mg, $447 \mu \mathrm{mol}, 100 \%$ ) was obtained as colorless oil.

The product was used without further purification in the next step. 


\section{3b) Syntheses of Precursors of Type 1}

\section{$N$-(3-(2-Bromophenoxy)-6,6-dimethylhept-4-yn-1-yl)aniline (1a)}<smiles>CC(C)(C)C#CC(CCNc1ccccc1)Oc1ccccc1Br</smiles>

The toslyate $15(1.42 \mathrm{~g}, 3.05 \mathrm{mmol})$ was reacted with potassium iodide $(54.2 \mathrm{mg}, 327 \mu \mathrm{mol})$, aniline $(420 \mu \mathrm{L}, 428 \mathrm{mg}, 4.60 \mathrm{mmol})$ and potassium carbonate $(1.26 \mathrm{~g}, 9.12 \mathrm{mmol})$ according to GP2 (Synthesis of Amine (A)). The reaction mixture was stirred $19 \mathrm{~h}$. Silica gel column chromatography (EtOAc: $n$-pentane = 1:20) afforded product 1a $(868 \mathrm{mg}, 2.25 \mathrm{mmol}, 73 \%)$ as yellow oil.

${ }^{1} \mathbf{H}-\mathrm{NMR}\left(300 \mathrm{MHz}, \mathrm{CDCl}_{3}\right): \delta=1.17(\mathrm{~s}, 9 \mathrm{H}), 2.23-2.31(\mathrm{~m}, 2 \mathrm{H}), 3.49(\mathrm{qt}, J=12.8,6.4 \mathrm{~Hz}$, 2H), $4.19(\mathrm{~s}, 1 \mathrm{H}), 4.90(\mathrm{t}, J=5.7 \mathrm{~Hz}, 1 \mathrm{H}), 6.64-6.72(\mathrm{~m}, 3 \mathrm{H}), 6.86(\mathrm{ddd}, J=7.9,7.2,1.7 \mathrm{~Hz}$, 1H), 7.13-7.20 (m, 3H), 7.25 (dd, $J=15.5,1.6 \mathrm{~Hz}, 1 \mathrm{H}), 7.54$ (dd, $J=7.9,1.6 \mathrm{~Hz}, 1 \mathrm{H})$.

${ }^{13} \mathbf{C}-\mathrm{NMR}\left(76 \mathrm{MHz}, \mathrm{CDCl}_{3}\right): \delta=27.6,30.9,35.6,40.4,68.8,76.0,97.2,112.9,113.2,116.2$, $117.3,122.7,128.3,129.4,133.3,148.4,154.3$.

IR (ATR): $\tilde{v}\left(\mathrm{~cm}^{-1}\right)=3412(\mathrm{w}), 2968(\mathrm{~m}), 2928(\mathrm{w}), 2899(\mathrm{w}), 2867(\mathrm{w}), 1603(\mathrm{~m}), 1507$ (m), $1475(\mathrm{~s}), 1239(\mathrm{~m})$.

HRMS-ESI: $\mathrm{C}_{21} \mathrm{H}_{24} \mathrm{BrNO} \mathrm{m} / z$ calcd. [M+Na ${ }^{+}$: 408.0933 found 408.0939 . 

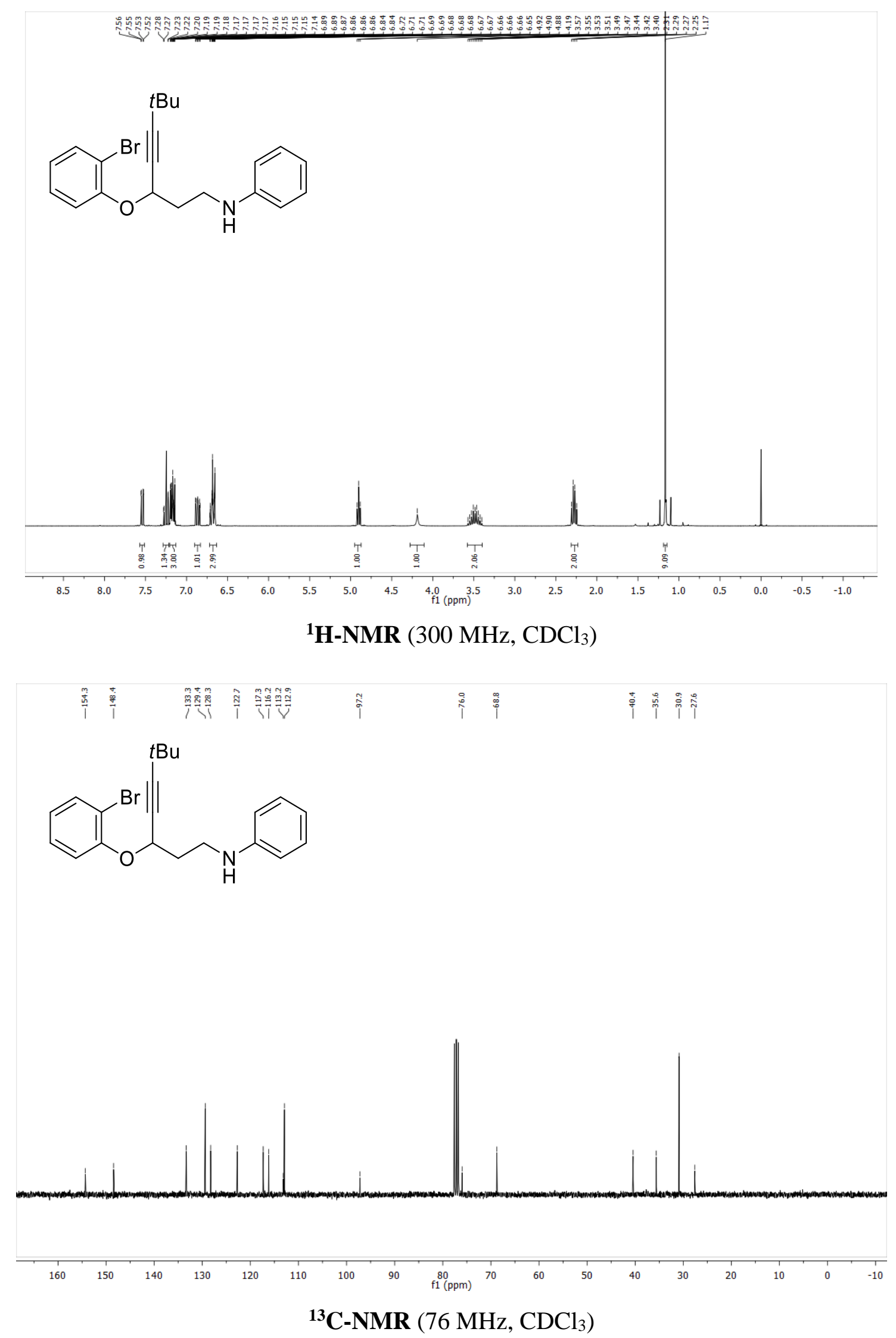


\section{$N$-(3-(2-Bromophenoxy)-5-(tert-butyldimethylsilyl)pent-4-yn-1-yl)aniline (1b)}<smiles>CC(C)(C)C#CC(CCNc1ccccc1)Oc1ccccc1Br</smiles>

The toslyate $17(1.16 \mathrm{~g}, 2.41 \mathrm{mmol})$ was reacted with potassium iodide $(52.0 \mathrm{mg}, 313 \mu \mathrm{mol})$, aniline $(350 \mu \mathrm{L}, 3.83 \mathrm{mmol})$ and potassium carbonate $(1.43 \mathrm{~g}, 6.74 \mathrm{mmol})$ according to $\mathrm{GP} 2$ (Synthesis of Amines (A)). The reaction mixture was stirred $16 \mathrm{~h}$ at rt. Silica gel column chromatography (EtOAc: $n$-pentane = 1:20) afforded terminal alkyne 20 (365 mg, 1.11 mmol, $46 \%$ ) as yellow oil.<smiles>C#CC(CCNc1ccccc1)Oc1ccccc1Br</smiles>

The terminal alkyne 20 (365 mg, $1.11 \mathrm{mmol})$ was dissolved in THF $(10 \mathrm{~mL})$. The reaction mixture was cooled to $-78^{\circ} \mathrm{C}$. Afterwards LDA ( $\left.1 \mathrm{M}, 1.30 \mathrm{~mL}, 1.30 \mathrm{mmol}\right)$ was added dropwise over $30 \mathrm{~min}$. The reaction mixture was stirred $1 \mathrm{~h}$ at $-78^{\circ} \mathrm{C}$. A solution of $\mathrm{TBSCl}$ (212 $\mathrm{mg}, 1.41 \mathrm{mmol})$ in THF (2.0 mL) was added dropwise over $10 \mathrm{~min}$ and the mixture was slowly warmed up to rt. The reaction was quenched with sat. $\mathrm{NH}_{4} \mathrm{Cl}$ solution. The aqueous layer was extracted with EtOAc (3x). The combined organic layers were dried over $\mathrm{Na}_{2} \mathrm{SO}_{4}$ and concentrated in vacuo. Silica gel column chromatography (EtOAc: $n$-pentane = 1:50) afforded product $\mathbf{1 b}(308 \mathrm{mg}, 693 \mu \mathrm{mol}, 62 \%)$ as yellow oil.

${ }^{1} \mathbf{H}-\mathbf{N M R}\left(400 \mathrm{MHz}, \mathrm{CDCl}_{3}\right): \delta=0.08(\mathrm{~s}, 6 \mathrm{H}), 0.87(\mathrm{~s}, 9 \mathrm{H}), 2.33(\mathrm{q}, J=6.3 \mathrm{~Hz}, 2 \mathrm{H}), 3.43-$ $3.63(\mathrm{~m}, 2 \mathrm{H}), 4.17(\mathrm{~s}, 1 \mathrm{H}), 4.94(\mathrm{t}, J=5.8 \mathrm{~Hz}, 1 \mathrm{H}), 6.64-6.75(\mathrm{~m}, 3 \mathrm{H}), 6.88(\mathrm{ddd}, J=7.9$, 7.3, 1.6 Hz, 1H), 7.14-7.22 (m, 3H), 7.22-7.28 (m, 1H), 7.55 (dd, $J=7.9,1.6 \mathrm{~Hz}, 1 \mathrm{H})$.

${ }^{13} \mathrm{C}-\mathrm{NMR}\left(101 \mathrm{MHz}, \mathrm{CDCl}_{3}\right): \delta=-4.9,-4.8,16.5,25.9,35.2,40.2,68.5,91.6,103.0,112.8$, $113.0,116.1,117.3,122.8,128.2,129.2,133.3,148.2,153.9$.

IR (ATR): $\tilde{v}\left(\mathrm{~cm}^{-1}\right)=3413(\mathrm{w}), 2952(\mathrm{~m}), 2934(\mathrm{~m}), 2890(\mathrm{w}), 2857(\mathrm{~m}), 1061(\mathrm{~m}), 1507$ (m), 1473 (s), 1243 (m), $1038(\mathrm{w})$.

HRMS-ESI: $\mathrm{C}_{23} \mathrm{H}_{30} \mathrm{BrNOSi} \mathrm{m} / z$ calcd. $\left[\mathrm{M}+\mathrm{H}^{+}\right]$: 444.1353 found 444.1364 . 


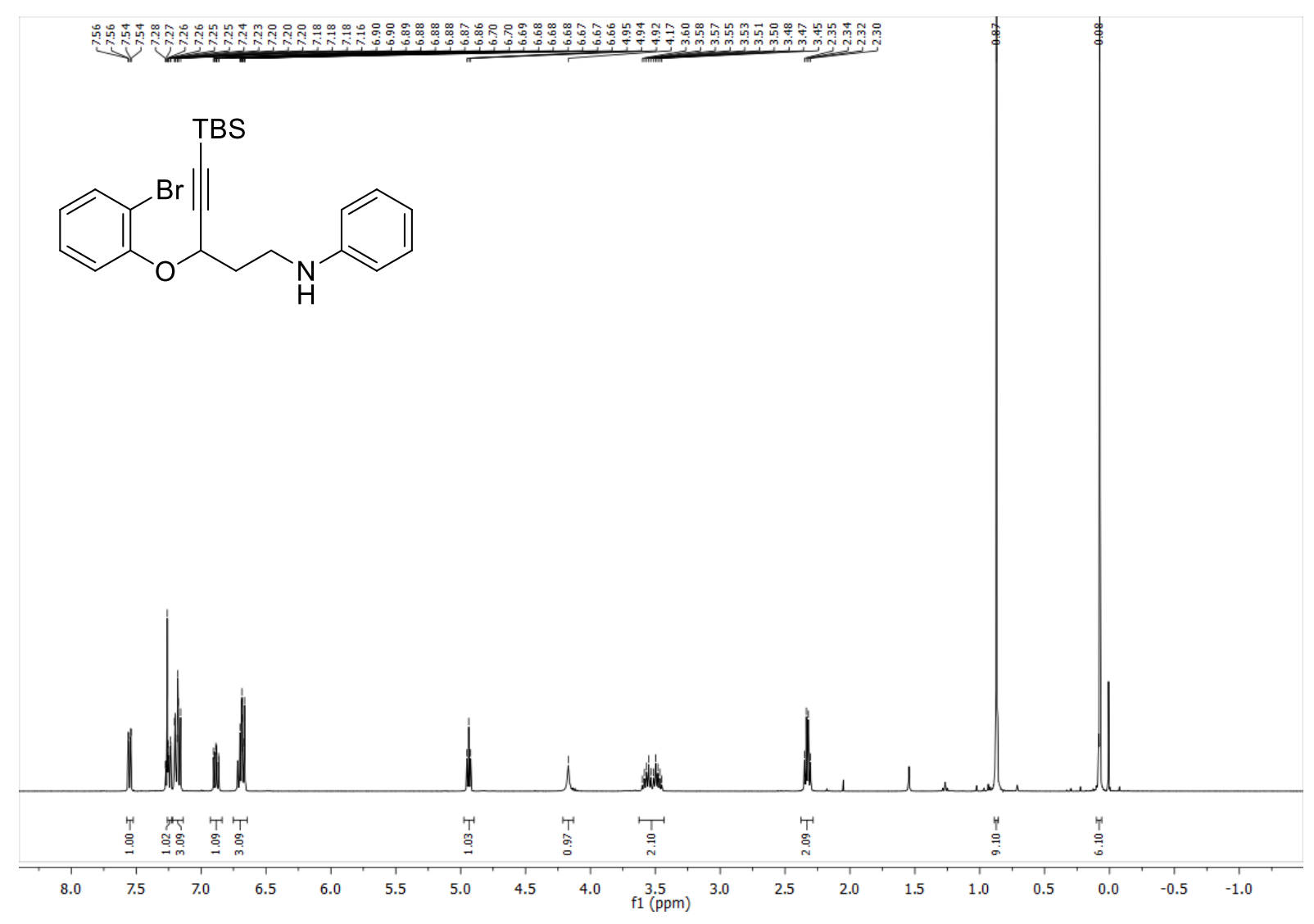

${ }^{1}$ H-NMR $\left(400 \mathrm{MHz}, \mathrm{CDCl}_{3}\right)$

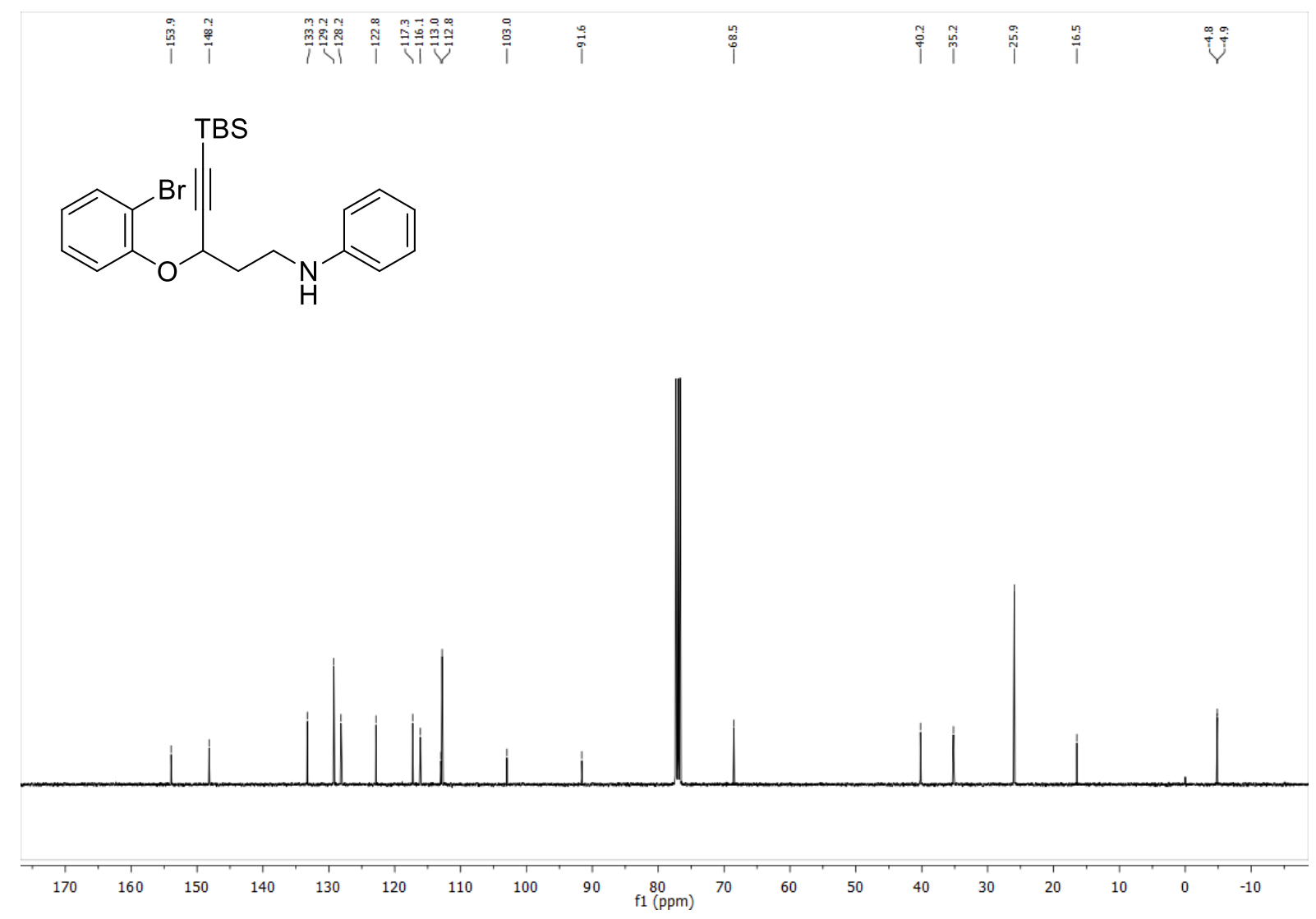

${ }^{13} \mathbf{C}-\mathbf{N M R}\left(101 \mathrm{MHz}, \mathrm{CDCl}_{3}\right)$ 
<smiles>COc1cc(NCCC(C#CC(C)(C)C)Oc2ccccc2Br)cc(OC)c1</smiles>

The tosylate $16(230 \mathrm{mg}, 495 \mu \mathrm{mol})$ was reacted with potassium iodide $(15 \mathrm{mg}, 90.4 \mu \mathrm{mol})$, aniline $(119 \mathrm{mg}, 777 \mu \mathrm{mol})$ and potassium carbonate $(219 \mathrm{mg}, 1.58 \mathrm{mmol})$ according to GP2 (Synthesis of Amine (A)). The reaction mixture was stirred $19 \mathrm{~h}$ at $90{ }^{\circ} \mathrm{C}$. Silica gel column chromatography (EtOAc: $n$-pentane = 1:10) afforded product 1c $(93.5 \mathrm{mg}, 209 \mu \mathrm{mol}, 42 \%)$ as yellow oil.

${ }^{1} \mathbf{H}-\mathbf{N M R}\left(300 \mathrm{MHz}, \mathrm{CDCl}_{3}\right): \delta=1.16(\mathrm{~s}, 9 \mathrm{H}), 2.27$ (q, $\left.J=6.1 \mathrm{~Hz}, 2 \mathrm{H}\right), 3.46(\mathrm{dtd}, J=19.0$, 13.0, $6.3 \mathrm{~Hz}, 2 \mathrm{H}), 3.74$ (s, 6H), 4.28 (s, 1H), 4.90 (t, J = 5.7 Hz, 1H), 5.86 (s, 3H), 6.81-6.93 (m, 1H), 7.12-7.26 (m, 2H), $7.54(\mathrm{dd}, J=7.9,1.6 \mathrm{~Hz}, 1 \mathrm{H})$.

${ }^{13}$ C-NMR $\left(75 \mathrm{MHz}, \mathrm{CDCl}_{3}\right) \delta=27.6,30.9,35.6,40.5,55.3,68.9,75.9,89.8,91.8,97.3$, $113.1,116.2,122.8,128.3,133.3,150.4,154.3,161.9$.

IR (ATR): $\tilde{v}\left(\mathrm{~cm}^{-1}\right)=3410(\mathrm{w}), 2966(\mathrm{~m}), 2931(\mathrm{w}), 2901(\mathrm{w}), 2867(\mathrm{w}), 2840(\mathrm{w}), 1614$ (m), $1591(\mathrm{~m}), 1474(\mathrm{~m}), 1239$ (m), 1201 (m), 1149 (s).

HRMS-ESI: $\mathrm{C}_{23} \mathrm{H}_{28} \mathrm{BrNO}_{3} \mathrm{~m} / z$ calcd. $\left[\mathrm{M}+\mathrm{H}^{+}\right]$: 446.1325 found 446.1336 . 


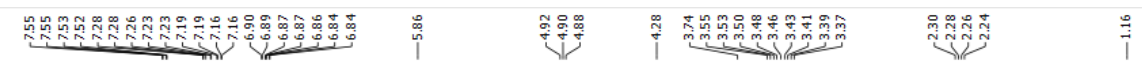<smiles>COc1cc(NCCC(C#CC(C)(C)C)Oc2ccccc2Br)cc(OC)c1</smiles>

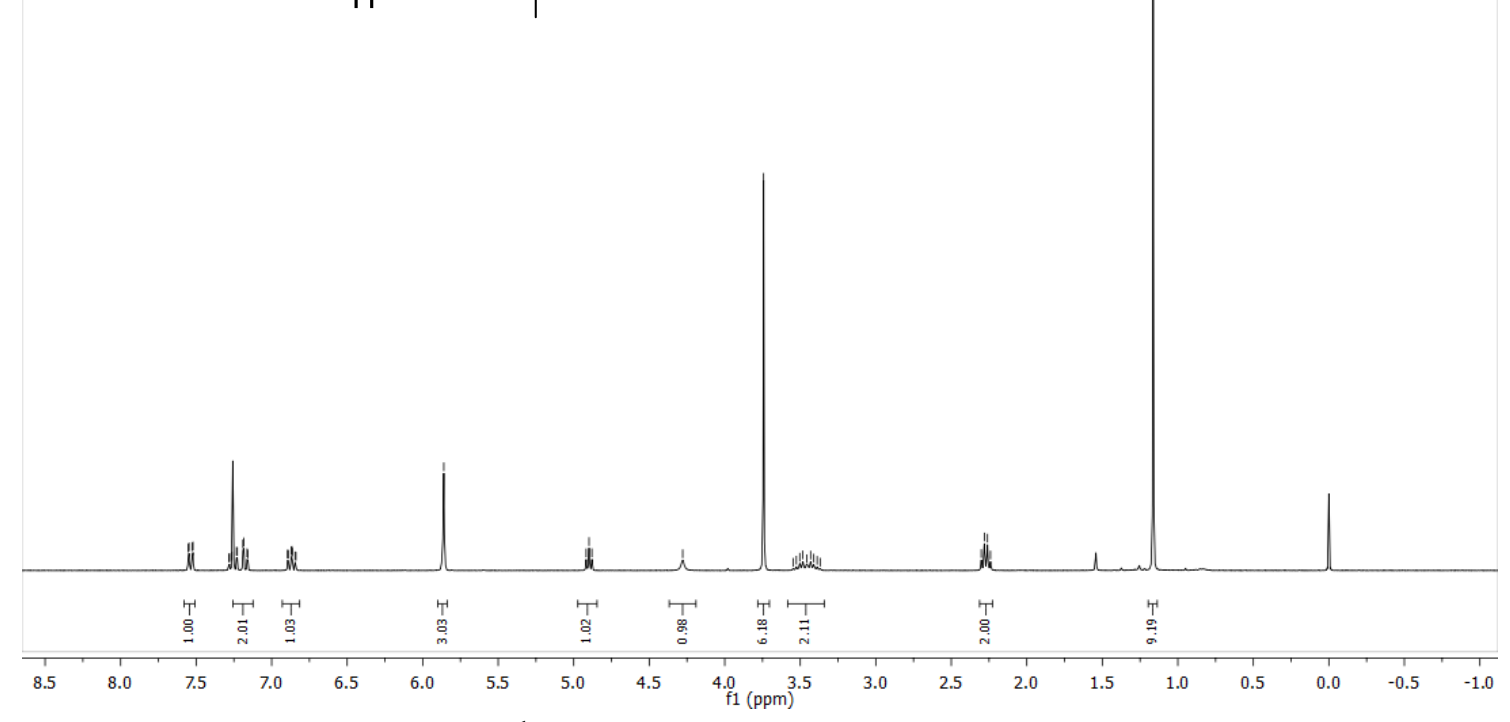

1'H-NMR (300 MHz, $\left.\mathrm{CDCl}_{3}\right)$

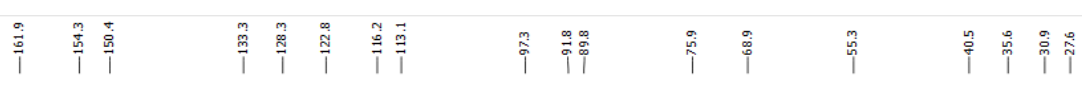<smiles>COc1cc(NCCC(C#CC(C)(C)C)Oc2ccccc2Br)cc(OC)c1</smiles>
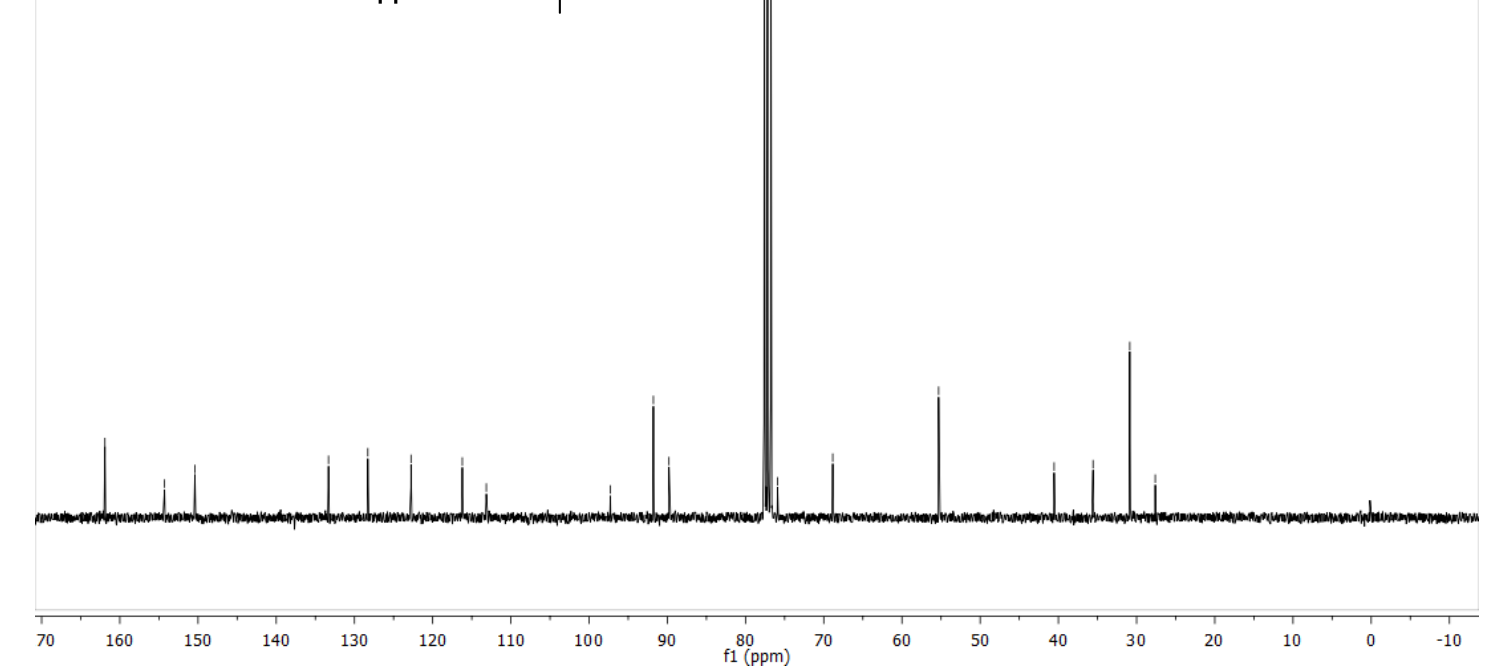

${ }^{13}$ C-NMR (75 MHz, $\left.\mathrm{CDCl}_{3}\right)$ 
<smiles>CC(C)(C)C#CC(CCNc1ccc(Cl)cc1)Oc1ccccc1Br</smiles>

The tosylate $16(261 \mathrm{mg}, 560 \mu \mathrm{mol})$ was reacted with potassium iodide $(30 \mathrm{mg}, 180 \mu \mathrm{mol})$, 4-chloroaniline (107 mg, $839 \mu \mathrm{mol})$ and potassium carbonate $(248 \mathrm{mg}, 1.79 \mathrm{mmol})$ according to GP2 (Synthesis of Amine (A)). The reaction mixture was stirred $14 \mathrm{~h}$ at $90{ }^{\circ} \mathrm{C}$. Silica gel column chromatography (EtOA: $n$-pentane = 1:50) afforded product $\mathbf{1 d}(53.4 \mathrm{mg}, 127 \mu \mathrm{mol}$, $23 \%$ ) as yellow oil.

${ }^{1} \mathbf{H}-\mathbf{N M R}\left(600 \mathrm{MHz}, \mathrm{CDCl}_{3}\right): \delta=1.16$ (s, 9H), 2.23-2.31 (m, 2H), 3.39-3.53 (m, 2H), 4.30 (s, 1H), $4.90(\mathrm{dd}, J=6.2,4.9 \mathrm{~Hz}, 1 \mathrm{H}), 6.57-6.61(\mathrm{~m}, 2 \mathrm{H}), 6.88(\mathrm{td}, J=7.6,1.5 \mathrm{~Hz}, 1 \mathrm{H})$, 7.09-7.13 (m, 2H), 7.17 (dd, $J=8.3,1.5 \mathrm{~Hz}, 1 \mathrm{H}), 7.24-7.27(\mathrm{~m}, 1 \mathrm{H}), 7.55$ (dd, $J=7.9,1.7$ $\mathrm{Hz}, 1 \mathrm{H})$.

${ }^{13}$ C-NMR $\left(151 \mathrm{MHz}, \mathrm{CDCl}_{3}\right) \delta=27.6,30.9,35.3,40.6,68.8,75.7,97.4,113.1,113.9,116.1$, $121.8,122.8,128.3,129.2,133.3,147.0,154.2$.

IR (ATR): $\tilde{v}\left(\mathrm{~cm}^{-1}\right)=3411(\mathrm{w}), 2969$ (m), 2928 (w), 2899 (w), 2867 (w), 2840 (w), 1601 (m), 1503 (m), 1475 (s), 1240 (m), 1049 (w), 1031 (w).

HRMS-ESI: $\mathrm{C}_{21} \mathrm{H}_{23} \mathrm{BrClNO} \mathrm{m} / \mathrm{z}$ calcd. $\left[\mathrm{M}+\mathrm{Na}^{+}\right]$: 442.0544 found 442.0547. 


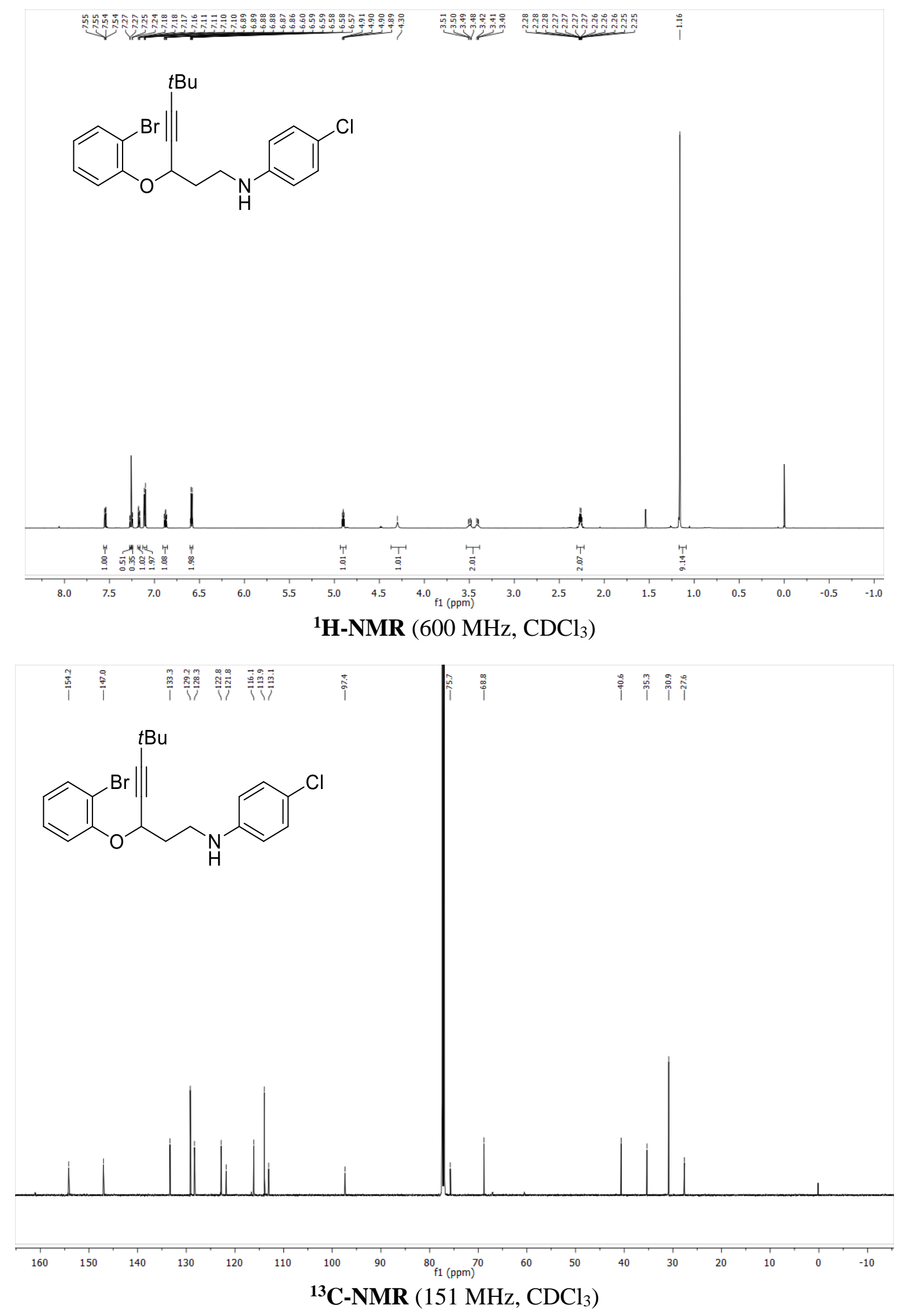


<smiles>CC(C)(C)C#CC(CCNc1ccc(F)cc1)Oc1ccccc1Br</smiles>

The tosylate $18(272 \mathrm{mg}, 520 \mu \mathrm{mol})$ was reacted with potassium iodide (36 mg, $217 \mu \mathrm{mol})$, 4-flouroaniline $(72 \mu \mathrm{L}, 750 \mu \mathrm{mol})$ and potassium phosphate $(222 \mathrm{mg}, 1.05 \mathrm{mmol})$ according to GP2 (Synthesis of Amine (A)). The reaction mixture was stirred $14 \mathrm{~h}$ at $90{ }^{\circ} \mathrm{C}$. Silica gel column chromatography (EtOAc: $n$-pentane $=1: 40)$ afforded product 1 e $(67.7 \mathrm{mg}, 146 \mu \mathrm{mol}$, $28 \%$ ) as yellow oil.

${ }^{1} \mathbf{H}-\mathrm{NMR}\left(300 \mathrm{MHz}, \mathrm{CDCl}_{3}\right): \delta=0.06(\mathrm{~s}, 6 \mathrm{H}), 0.86(\mathrm{~s}, 9 \mathrm{H}), 2.24-2.38(\mathrm{~m}, 2 \mathrm{H}), 3.36-3.64$ $(\mathrm{m}, 2 \mathrm{H}), 4.11(\mathrm{~s}, 1 \mathrm{H}), 4.94(\mathrm{t}, J=5.7 \mathrm{~Hz}, 1 \mathrm{H}), 6.55-6.64(\mathrm{~m}, 2 \mathrm{H}), 6.82-6.92(\mathrm{~m}, 3 \mathrm{H}), 7.15-$ $7.28(\mathrm{~m}, 2 \mathrm{H}), 7.55(\mathrm{dd}, J=7.9,1.6 \mathrm{~Hz}, 1 \mathrm{H})$.

${ }^{13}$ C-NMR (76 MHz, $\left.\mathrm{CDCl}_{3}\right) \delta=-4.9,-4.8,16.5,25.9,35.0,40.9,68.6,91.7,102.8,112.9$, $113.59(\mathrm{~d}, J=7.4 \mathrm{~Hz}), 115.91(\mathrm{~d}, J=20.6 \mathrm{~Hz}), 116.0,122.9,128.2,133.3,144.56$ (d, $J=1.8 \mathrm{~Hz}), 153.9,154.2$.

${ }^{19}$ F-NMR $\left(283 \mathrm{MHz}, \mathrm{CDCl}_{3}\right): \delta=-128.78$.

IR (ATR): $\tilde{v}\left(\mathrm{~cm}^{-1}\right)=3410(\mathrm{w}), 2952(\mathrm{~m}), 2934$ (m), 2890 (w), 2857 (m), $1513(\mathrm{~s}), 1473$ (m), $1228(\mathrm{~m}), 1039(\mathrm{~m})$.

ESI-HRMS: $\mathrm{C}_{23} \mathrm{H}_{29} \mathrm{BrFNOSi} \mathrm{m} / \mathrm{z}$ calcd. $\left[\mathrm{M}+\mathrm{H}^{+}\right]$: 462.1259 found 462.1264 . 
<smiles>CC(C)(C)C#CC(CCNc1ccc(F)cc1)Oc1ccccc1Br</smiles>

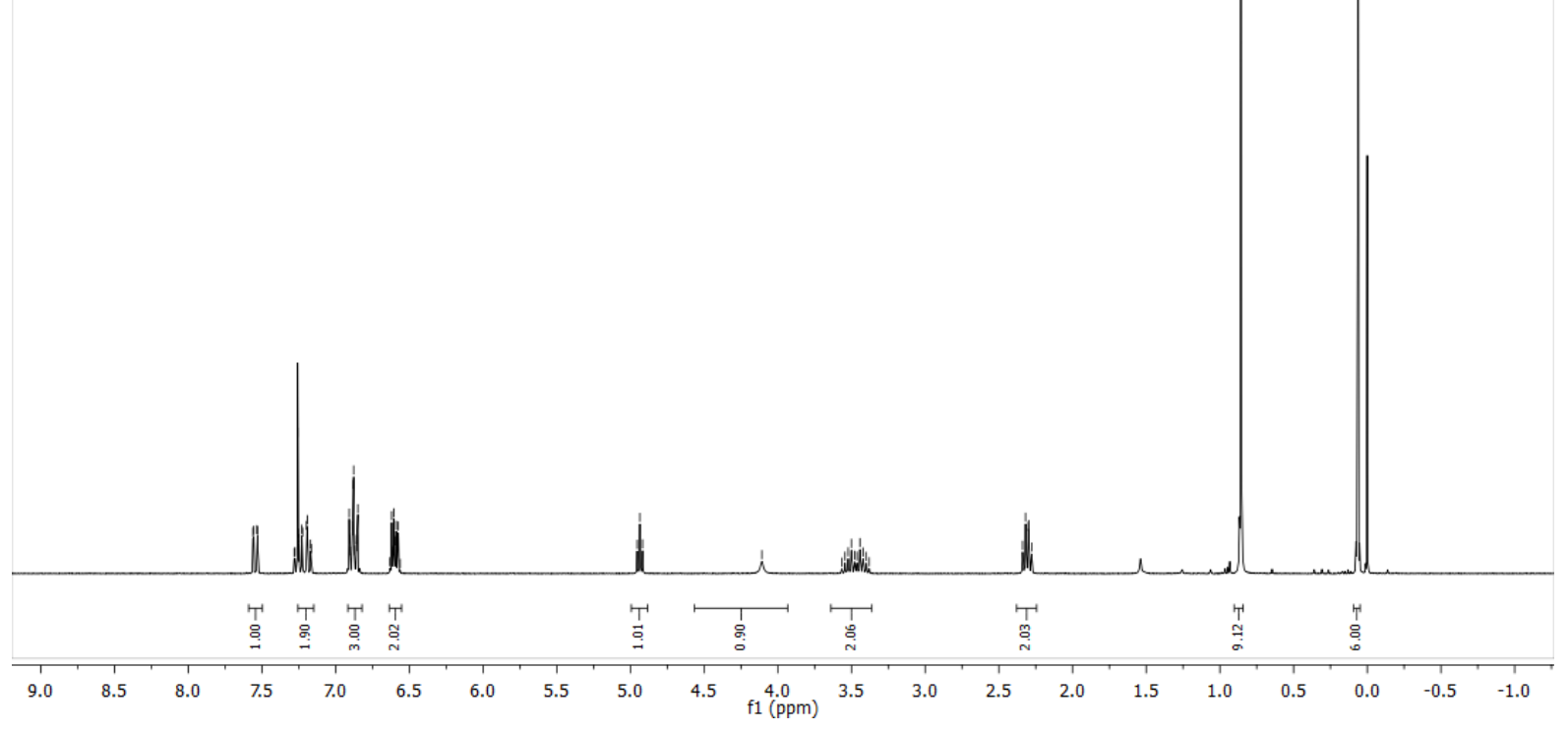

${ }^{1} \mathbf{H}-\mathbf{N M R}\left(300 \mathrm{MHz}, \mathrm{CDCl}_{3}\right)$

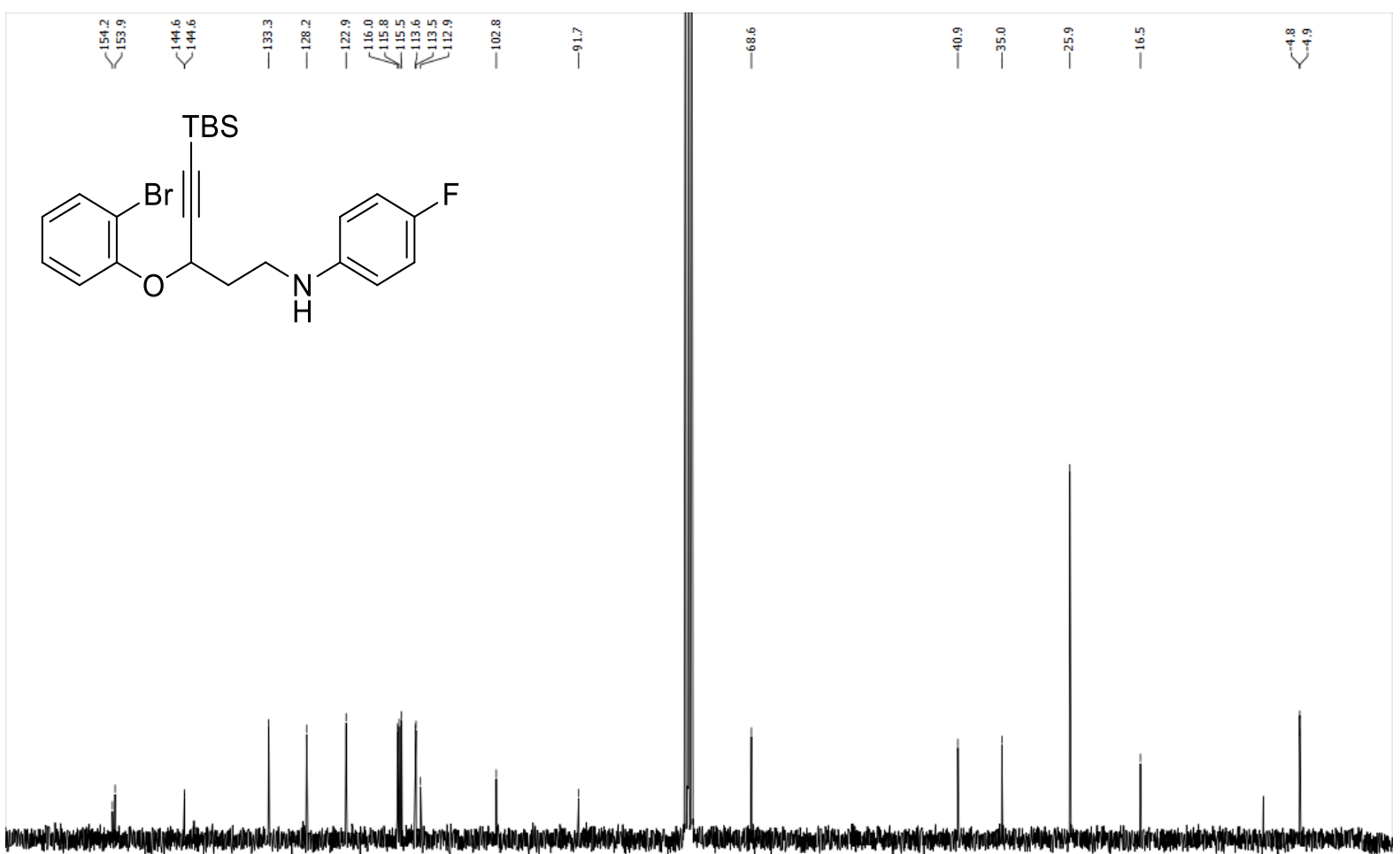

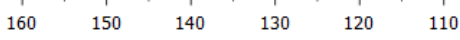

${ }^{\mathbf{1 3}} \mathbf{C}$-NMR (76 MHz, $\left.\mathrm{CDCl}_{3}\right)$ 


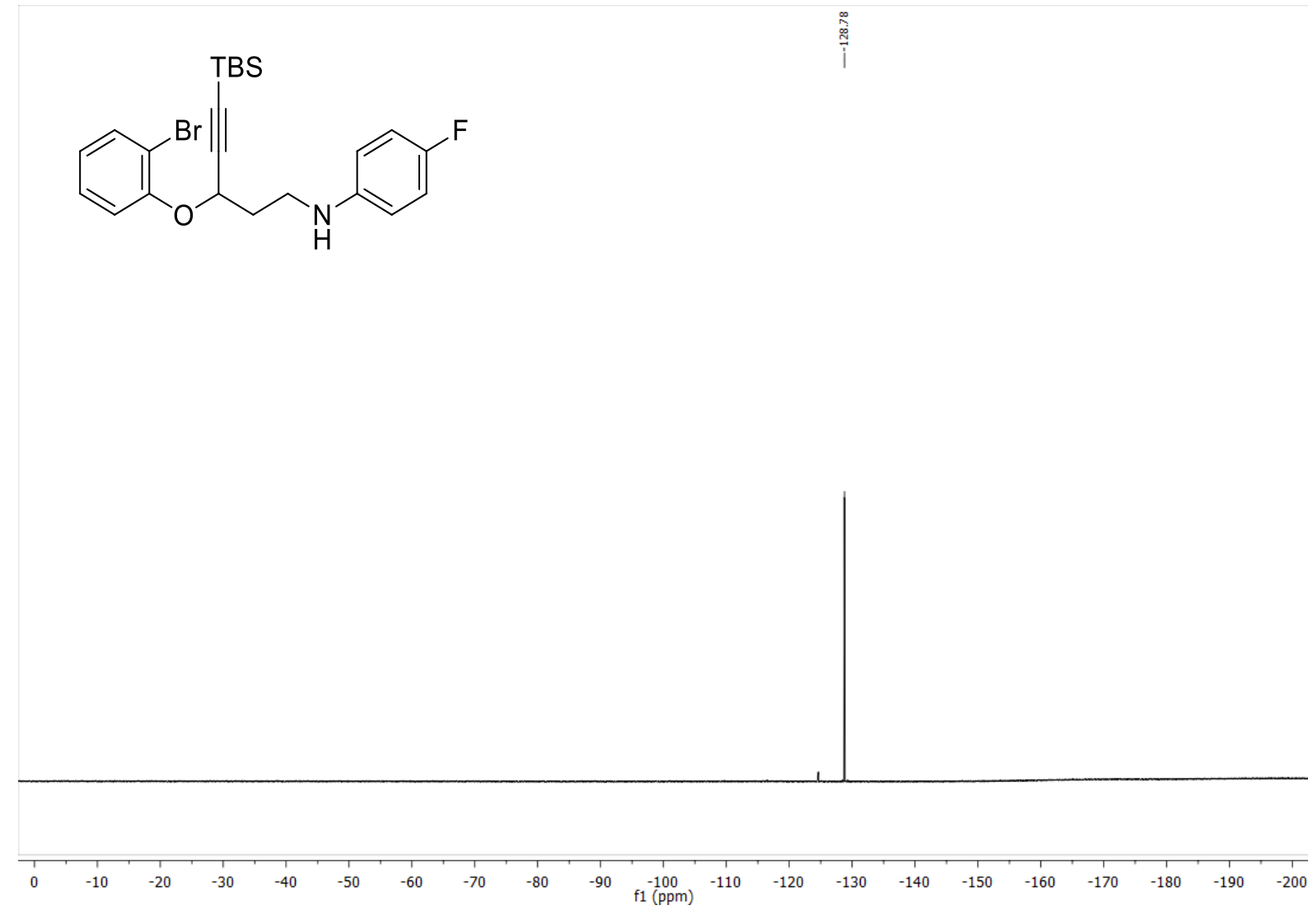

${ }^{19}$ F-NMR $\left(283 \mathrm{MHz}, \mathrm{CDCl}_{3}\right)$ 
<smiles>CC(C)(C)C#CC(CCNc1ccccc1)Oc1cc2ccccc2cc1Br</smiles>

The tosylate $19(230 \mathrm{mg}, 447 \mu \mathrm{mol})$ was reacted with potassium iodide $(20 \mathrm{mg}, 120 \mu \mathrm{mol})$, aniline $(100 \mu \mathrm{L}, 102 \mathrm{mg}, 1.10 \mathrm{mmol})$ and potassium carbonate $(136 \mathrm{mg}, 984 \mu \mathrm{mol})$ according to GP2 (Synthesis of Amine (A)). The reaction mixture was stirred $19 \mathrm{~h}$ at $90{ }^{\circ} \mathrm{C}$. Silica gel column chromatography (EtOAc: $n$-pentane = 1:20) afforded product $\mathbf{1 f}(39.2 \mathrm{mg}, 89.8 \mathrm{mmol}$, $20 \%$ ) as colorless oil.

${ }^{1} \mathbf{H}-\mathbf{N M R}\left(300 \mathrm{MHz}, \mathrm{CDCl}_{3}\right): \delta=1.18(\mathrm{~s}, 9 \mathrm{H}), 2.35(\mathrm{q}, J=6.3 \mathrm{~Hz}, 2 \mathrm{H}), 3.53(\mathrm{tt}, J=12.9$, $6.5 \mathrm{~Hz}, 2 \mathrm{H}), 4.23(\mathrm{~s}, 1 \mathrm{H}), 5.03(\mathrm{t}, J=5.7 \mathrm{~Hz}, 1 \mathrm{H}), 6.66-6.74(\mathrm{~m}, 3 \mathrm{H}), 7.18(\mathrm{dd}, J=8.6,7.3$ $\mathrm{Hz}, 2 \mathrm{H}), 7.34-7.51(\mathrm{~m}, 3 \mathrm{H}), 7.71(\mathrm{~d}, J=8.2 \mathrm{~Hz}, 2 \mathrm{H}), 8.07$ (s, 1H).

${ }^{13}$ C-NMR (126 MHz, $\left.\mathrm{CDCl}_{3}\right): \delta=27.7,30.9,35.6,40.5,68.7,75.9,97.6,110.8,112.9$, $114.3,117.4,124.9,126.8,126.9,127.0,129.4,129.9,132.2,133.5,148.4,151.7$.

IR (ATR): $\tilde{v}\left(\mathrm{~cm}^{-1}\right)=3411(\mathrm{w}), 2964(\mathrm{~m}), 2923(\mathrm{~m}), 2853(\mathrm{~m}), 1602(\mathrm{~m}), 1506$ (m), 1455 (s), $1244(\mathrm{~m}), 1215(\mathrm{~m}), 1182(\mathrm{~m})$.

HRMS-ESI: $\mathrm{C}_{25} \mathrm{H}_{26} \mathrm{BrNO} \mathrm{m} / \mathrm{z}$ calcd. [M+H $\left.{ }^{+}\right]$: 436.1271 found 436.1278. 


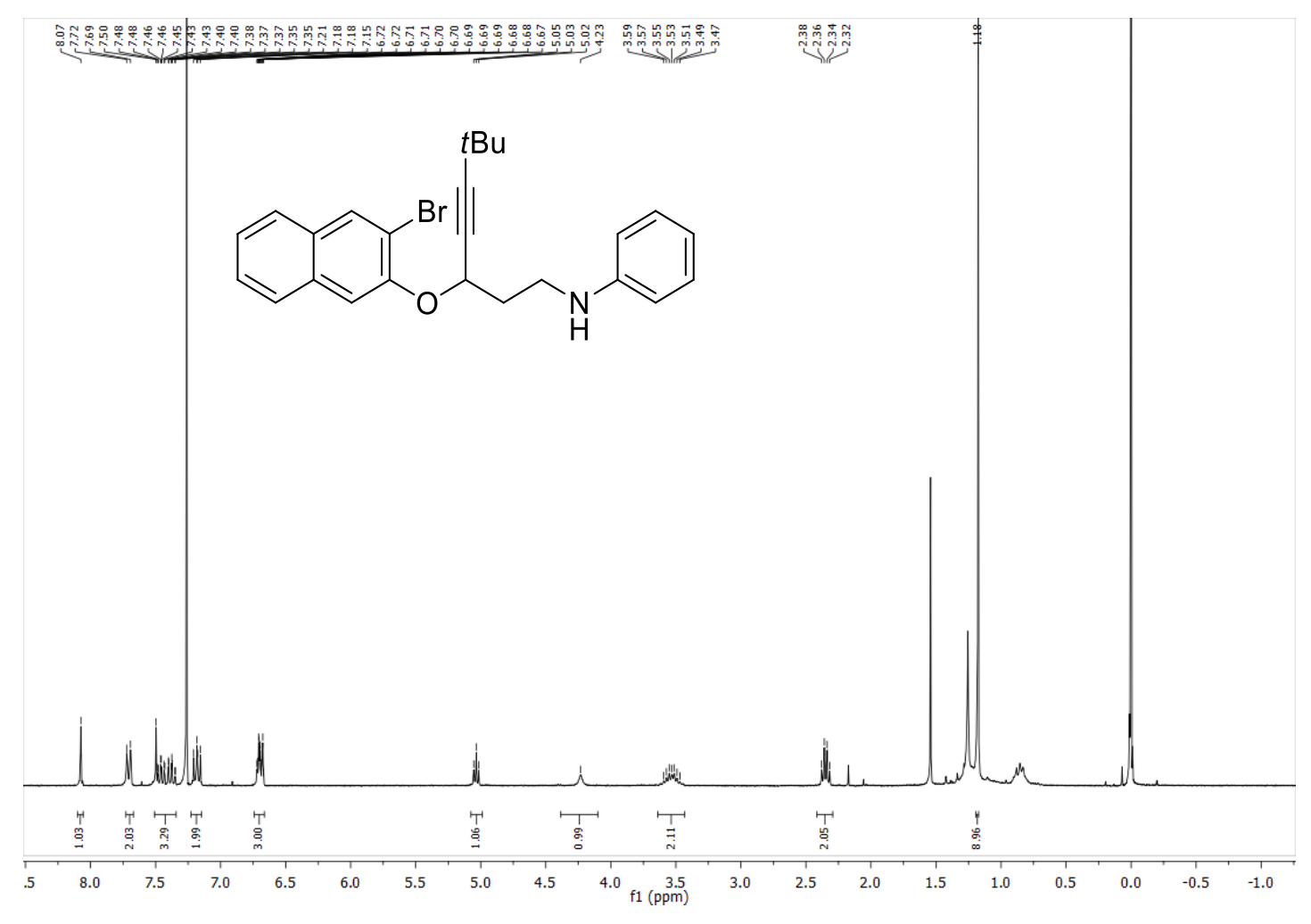

${ }^{\mathbf{1}} \mathbf{H}$-NMR (300 MHz, $\mathrm{CDCl}_{3}$ )

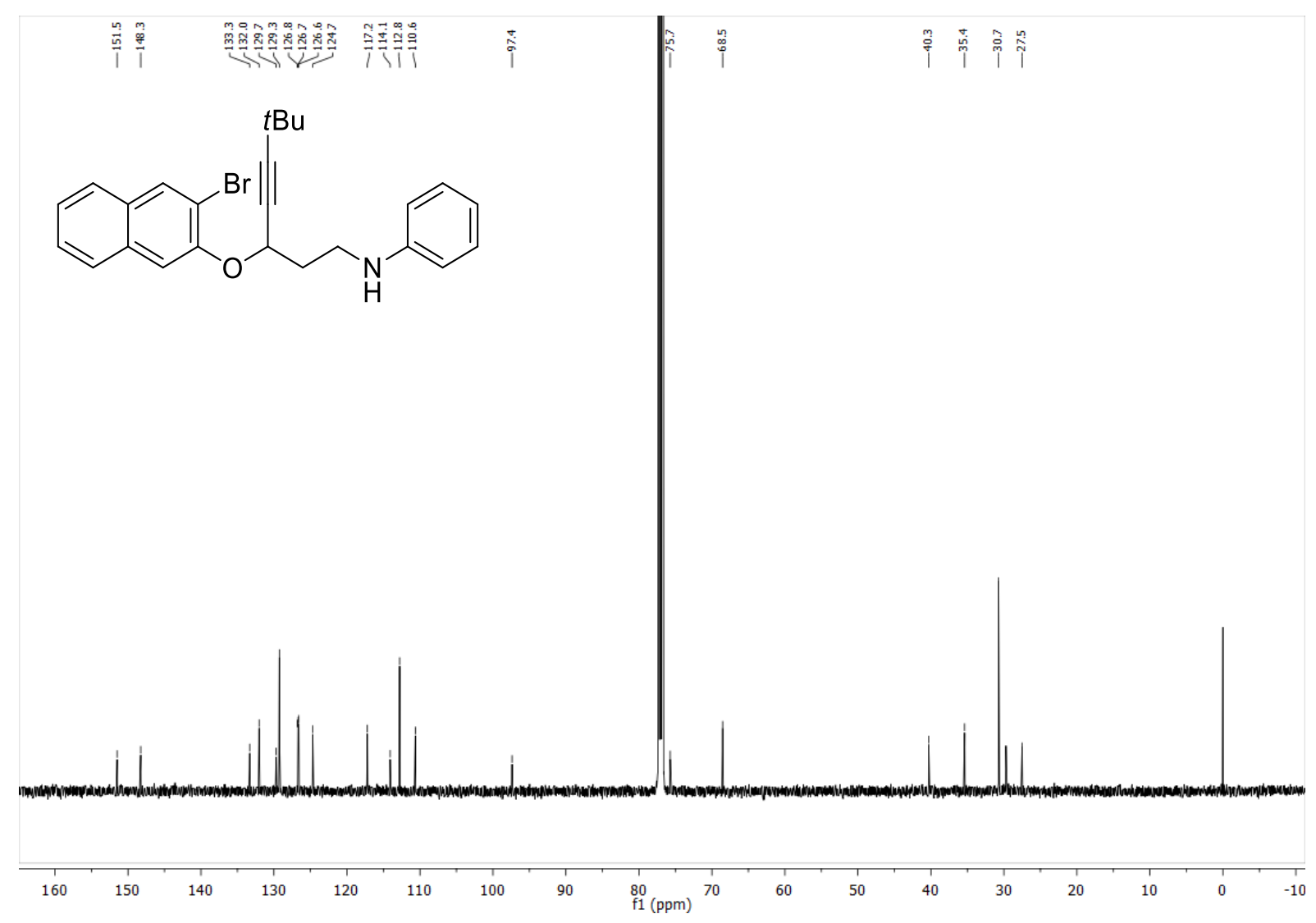

${ }^{13}$ C-NMR (126 MHz, $\left.\mathrm{CDCl}_{3}\right)$ 


\section{3c) Syntheses of Domino Products of Type 3}

\section{Domino product 3a}<smiles>CC(C)(C)C1=C2c3ccccc3OC2CCN1c1ccccc1</smiles>

The domino precursor 1a $(38.3 \mathrm{mg}, 99.1 \mu \mathrm{mol})$, potassium phosphate $(105 \mathrm{mg}, 495 \mu \mathrm{mol})$, $\left[t \mathrm{Bu}_{3} \mathrm{PH}\right]\left[\mathrm{BF}_{4}\right](3.0 \mathrm{mg}, 10.3 \mu \mathrm{mol})$ and $\mathrm{Pd}(\mathrm{dba})_{2}(3.5 \mathrm{mg}, 6.1 \mu \mathrm{mol})$ were reacted according to GP3 (Pd-catalyzed Domino Reaction). The mixture was stirred $2 \mathrm{~h}$ at $120{ }^{\circ} \mathrm{C}$. Silica gel column chromatography (EtOAc: $n$-pentane $=1: 20)$ afforded domino product 3a $(19.6 \mathrm{mg}$, $90.6 \mu \mathrm{mol}, 85 \%)$ as yellow solid.

${ }^{1}$ H-NMR (300 MHz, Acetone- $\left.d_{6}\right): \delta=1.32(\mathrm{~s}, 9 \mathrm{H}), 1.88(\mathrm{dtd}, J=12.2,8.9,5.5 \mathrm{~Hz}, 1 \mathrm{H})$, 2.76-2.85 (m, 1H), 3.15 (ddd, $J=12.5,8.8,6.3 \mathrm{~Hz}, 1 \mathrm{H}), 3.82(\mathrm{ddd}, J=12.5,8.7,5.5 \mathrm{~Hz}$, $1 \mathrm{H}), 4.70(\mathrm{dd}, J=9.1,7.3 \mathrm{~Hz}, 1 \mathrm{H}), 6.80-6.90(\mathrm{~m}, 2 \mathrm{H}), 6.91-7.04(\mathrm{~m}, 3 \mathrm{H}), 7.21(\mathrm{ddd}, J=8.0$, 7.3, $1.3 \mathrm{~Hz}, 3 \mathrm{H}), 7.75-7.84(\mathrm{~m}, 1 \mathrm{H})$.

${ }^{13}$ C-NMR (76 MHz, Acetone- $\left.d_{6}\right) \delta=29.0,36.1,37.7,45.9,81.4,111.1,118.3,120.8,121.3$, $123.8,127.4,129.6,129.9,131.7,144.9,150.4,164.1$.

IR (ATR): $\tilde{v}\left(\mathrm{~cm}^{-1}\right)=2955(\mathrm{w}), 2932(\mathrm{w}), 2871(\mathrm{w}), 1595$ (m), 1492 (m), 1459 (m), 1330 (m), $1225(\mathrm{~m}), 1215(\mathrm{~m})$.

M.p.: $91 \neg 96^{\circ} \mathrm{C}$.

GC-HRMS (EI): $\mathrm{C}_{21} \mathrm{H}_{23} \mathrm{NO} m / z$ calcd. $\left[\mathrm{M}^{+}\right]$: 305.1780 found 305.1797 . 

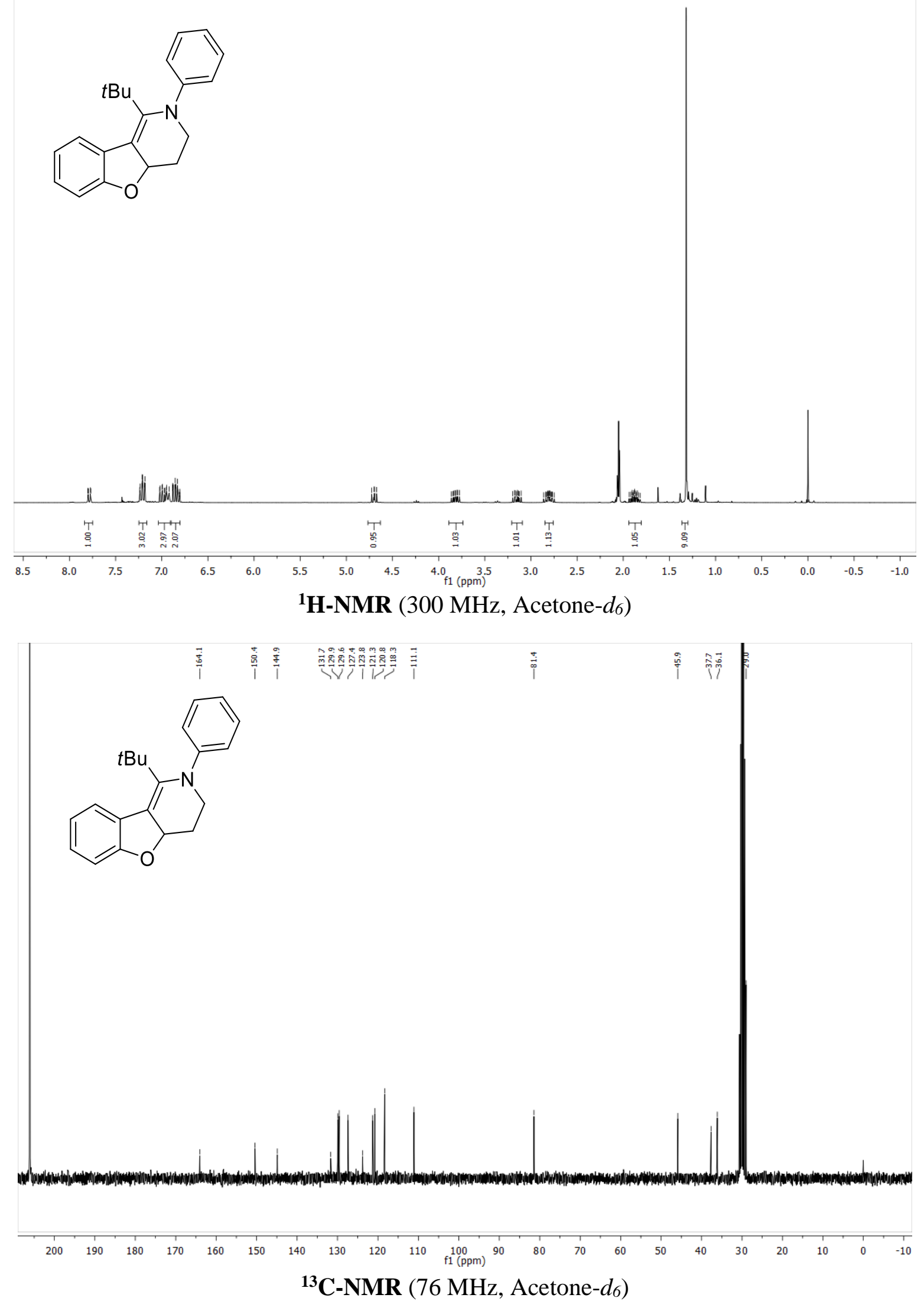


\section{Domino product 3b}<smiles>CC(C)(C)C1=C2c3ccccc3OC2CCN1c1ccccc1</smiles>

The domino precursor 1b $(47.2 \mathrm{mg}, 106 \mu \mathrm{mol})$, potassium phosphate $(105 \mathrm{mg}, 495 \mu \mathrm{mol})$, $\left[t \mathrm{Bu}_{3} \mathrm{PH}\right]\left[\mathrm{BF}_{4}\right](2.9 \mathrm{mg}, 10.0 \mu \mathrm{mol})$ and $\mathrm{Pd}(\mathrm{dba})_{2}(3.0 \mathrm{mg}, 5.2 \mu \mathrm{mol})$ were reacted according to GP3 (Pd-catalyzed Domino Reaction). The mixture was stirred $2 \mathrm{~h}$ at $120{ }^{\circ} \mathrm{C}$. Silica gel column chromatography (EtOAc: $n$-pentane $=1: 50)$ afforded domino product $\mathbf{3 b}(18.1 \mathrm{mg}$, $49.8 \mu \mathrm{mol}, 47 \%)$ as yellow oil.

${ }^{1} \mathbf{H}-\mathbf{N M R}\left(300 \mathrm{MHz}, \mathrm{CDCl}_{3}\right): \delta=-0.20(\mathrm{~s}, 3 \mathrm{H}), 0.44(\mathrm{~s}, 3 \mathrm{H}), 0.87(\mathrm{~s}, 9 \mathrm{H}), 1.87-2.04(\mathrm{~m}$, 1H), 2.82-2.97 (m, 1H), 3.37 (ddd, $J=11.9,8.8,3.7 \mathrm{~Hz}, 1 \mathrm{H}), 3.61(\mathrm{dt}, J=11.9,8.6 \mathrm{~Hz}, 1 \mathrm{H})$, $4.76(\mathrm{dd}, J=10.6,6.1 \mathrm{~Hz}, 1 \mathrm{H}), 6.81-6.95(\mathrm{~m}, 5 \mathrm{H}), 7.16-7.23(\mathrm{~m}, 3 \mathrm{H}), 7.71(\mathrm{dd}, J=7.8$, $1.3 \mathrm{~Hz}, 1 \mathrm{H})$.

${ }^{13}$ C-NMR $\left(76 \mathrm{MHz}, \mathrm{CDCl}_{3}\right) \delta=-3.6,-3.1,20.0,27.6,35.9,43.5,81.3,110.5,117.4,119.5$, $120.3,123.8,124.4,128.9,129.4,135.6,147.5,150.4,163.3$.

IR (ATR): $\tilde{v}\left(\mathrm{~cm}^{-1}\right)=2951(\mathrm{~m}), 2933(\mathrm{~m}), 2858(\mathrm{~m}), 1596(\mathrm{~m}), 1492(\mathrm{~m}), 1460(\mathrm{~m}), 1218$ (m), $1035(\mathrm{~m})$.

GC-HRMS (EI): $\mathrm{C}_{23} \mathrm{H}_{29} \mathrm{NOSi} \mathrm{m} / z$ calcd. $\left[\mathrm{M}^{+}\right]$: 363.2018 found 363.2037. 


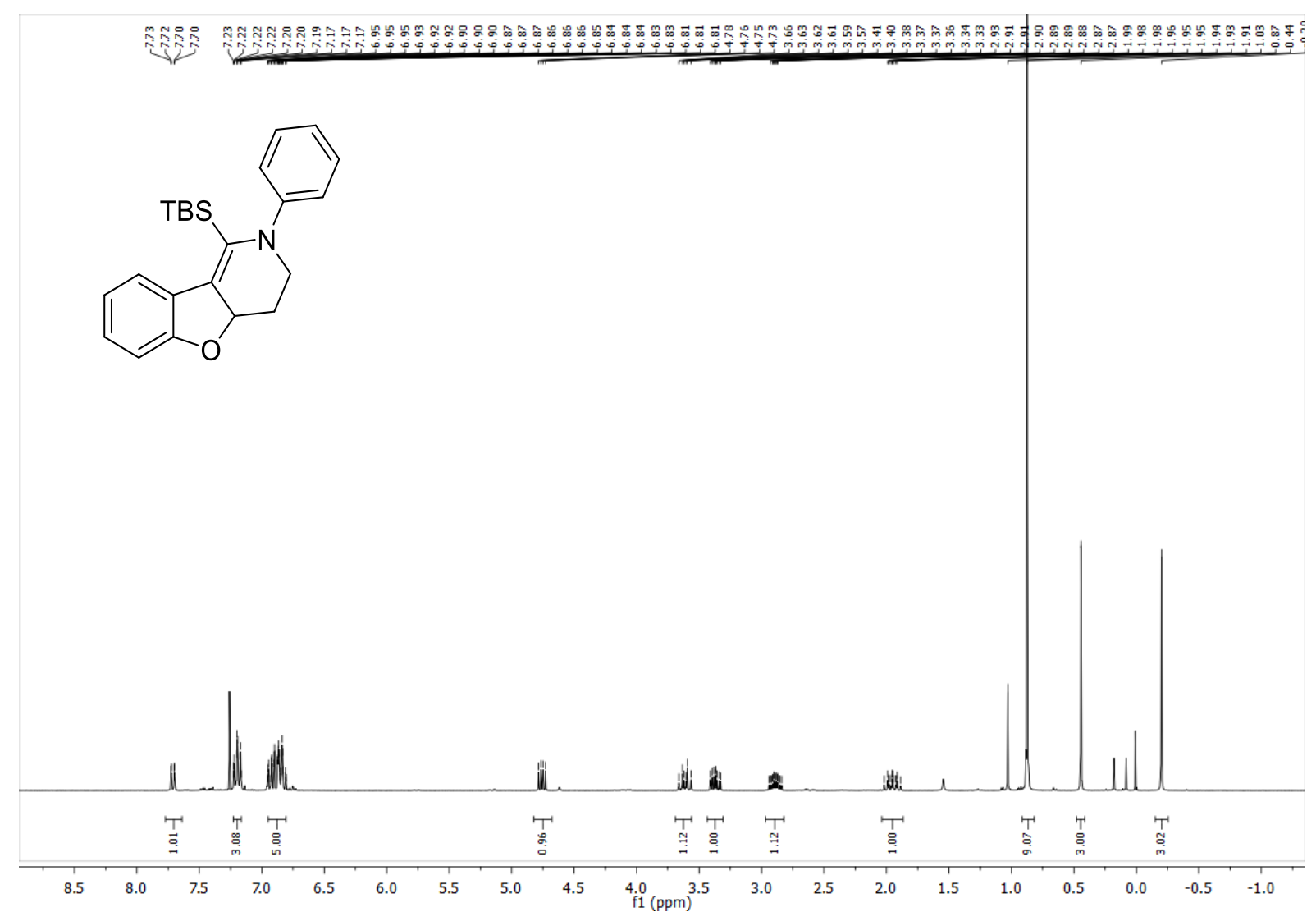

${ }^{1} \mathbf{H}-\mathbf{N M R}\left(300 \mathrm{MHz}, \mathrm{CDCl}_{3}\right)$

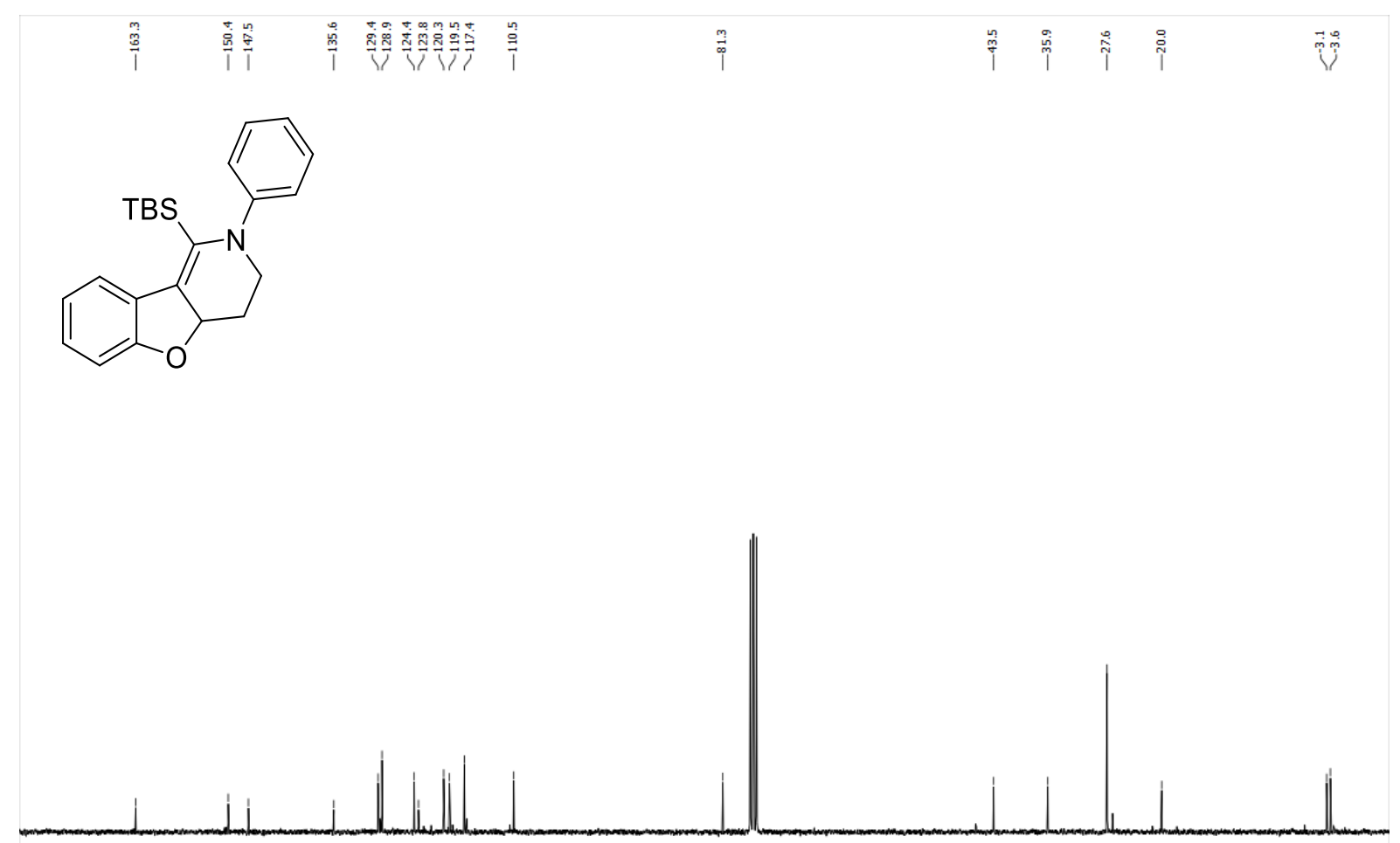

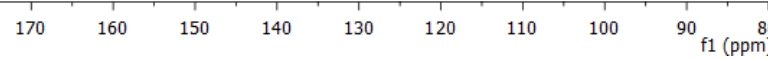

${ }^{13} \mathbf{C}-\mathrm{NMR}\left(76 \mathrm{MHz}, \mathrm{CDCl}_{3}\right)$ 


\section{Domino product 3c}

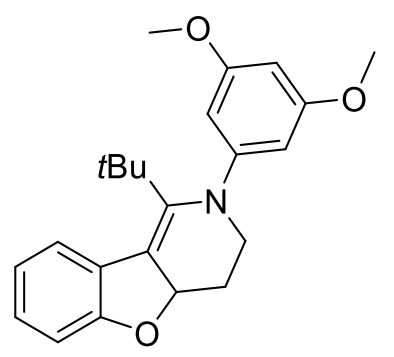

The domino precursor 1c $(43.5 \mathrm{mg}, 97.4 \mu \mathrm{mol})$, potassium phosphate $(100 \mathrm{mg}, 471 \mu \mathrm{mol})$, $\left[t \mathrm{Bu}_{3} \mathrm{PH}\right]\left[\mathrm{BF}_{4}\right](2.7 \mathrm{mg}, 9.3 \mu \mathrm{mol})$ and $\mathrm{Pd}(\mathrm{dba})_{2}(2.9 \mathrm{mg}, 5.0 \mu \mathrm{mol})$ were reacted according to GP3 (Pd-catalyzed Domino Reaction). The mixture was stirred $2 \mathrm{~h}$ at $120{ }^{\circ} \mathrm{C}$. Silica gel column chromatography (EtOAc: $n$-pentane $=1: 50)$ afforded domino product $3 \mathbf{c}(31.5 \mathrm{mg}$, $86.2 \mu \mathrm{mol}, 88 \%)$ as yellow solid.

${ }^{1}$ H-NMR (500 MHz, Acetone- $\left.d_{6}\right): \delta=1.35(\mathrm{~s}, 9 \mathrm{H}), 1.81-1.89(\mathrm{~m}, 1 \mathrm{H}), 2.78-2.82(\mathrm{~m}, 1 \mathrm{H})$, 3.09 (ddd, $J=12.6,8.9,6.3 \mathrm{~Hz}, 1 \mathrm{H}), 3.73$ (s, 6H), 3.85 (ddd, $J=12.7,8.8,5.4 \mathrm{~Hz}, 1 \mathrm{H}), 4.72$ $(\mathrm{dd}, J=9.2,7.3 \mathrm{~Hz}, 1 \mathrm{H}), 6.02(\mathrm{t}, J=2.1 \mathrm{~Hz}, 1 \mathrm{H}), 6.12(\mathrm{~s}, 2 \mathrm{H}), 6.87(\mathrm{dd}, J=8.1,1.2 \mathrm{~Hz}, 1 \mathrm{H})$, $7.00(\mathrm{td}, J=7.5,1.0 \mathrm{~Hz}, 1 \mathrm{H}), 7.19-7.25(\mathrm{~m}, 1 \mathrm{H}), 7.78(\mathrm{dd}, J=8.0,1.3 \mathrm{~Hz}, 1 \mathrm{H})$.

${ }^{13}$ C-NMR (126 MHz, Acetone- $\left.d_{6}\right) \delta=28.9,35.9,37.7,45.4,55.4,81.4,93.2,96.7,111.1$, $121.3,123.7,127.5,129.7,132.1,144.7,152.3,164.2$.

IR (ATR): $\tilde{v}\left(\mathrm{~cm}^{-1}\right)=2957(\mathrm{~m}), 2874(\mathrm{w}), 2842(\mathrm{w}), 1593(\mathrm{~s}), 1459(\mathrm{~m}), 1205(\mathrm{~m}), 1156(\mathrm{~m})$. M.p.: $99 \neg 102{ }^{\circ} \mathrm{C}$.

GC-HRMS (EI): $\mathrm{C}_{23} \mathrm{H}_{27} \mathrm{NO}_{3} \mathrm{~m} / \mathrm{z}$ calcd. $\left[\mathrm{M}^{+}\right]$: 365.1991 found 365.1971. 

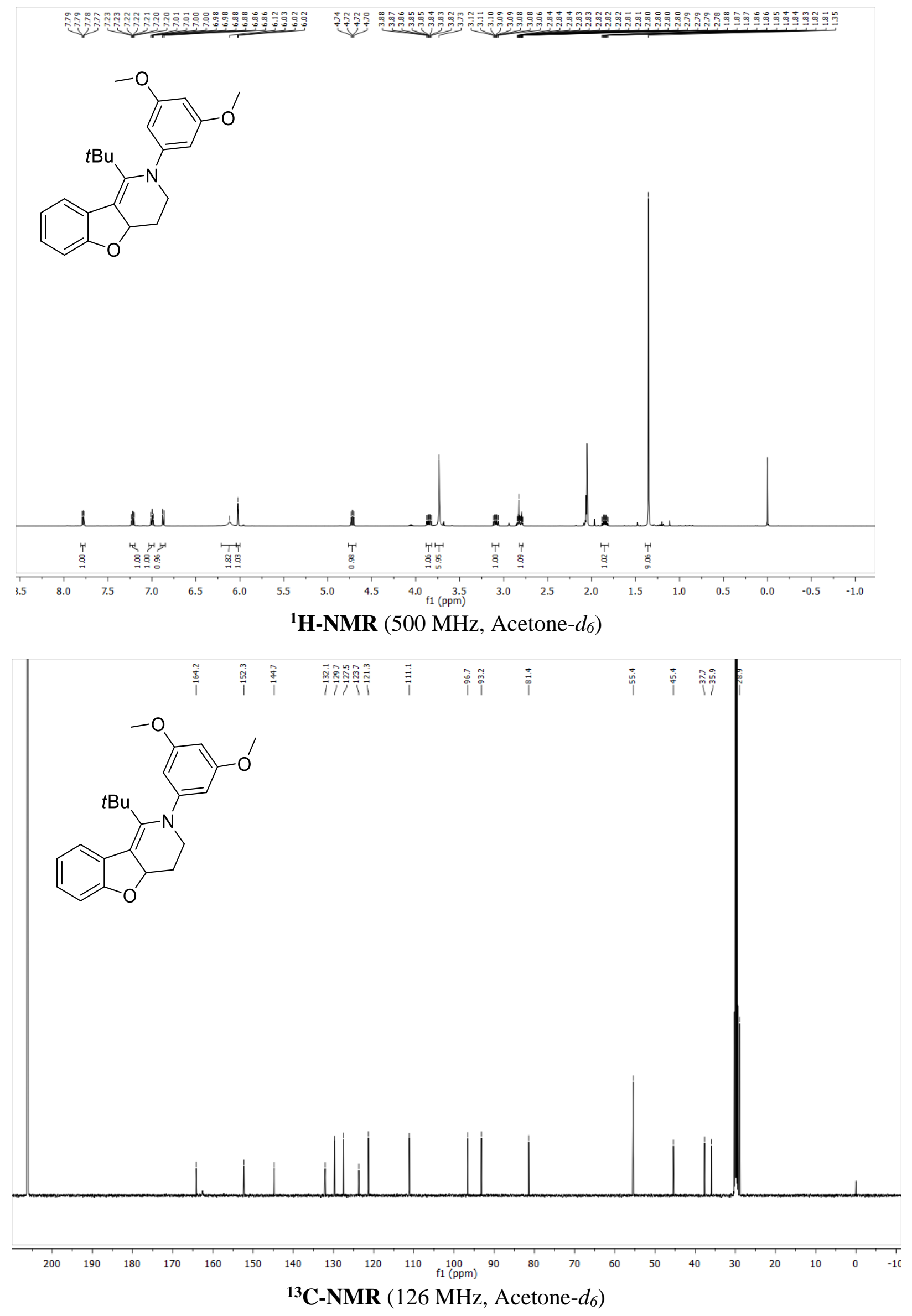


\section{Domino product 3d}

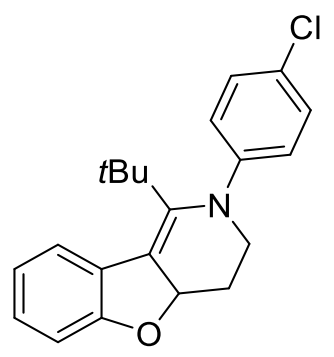

The domino precursor 1d $(45.6 \mathrm{mg}, 108 \mu \mathrm{mol})$, potassium phosphate $(106 \mathrm{mg}, 499 \mu \mathrm{mol})$, $\left[t \mathrm{Bu}_{3} \mathrm{PH}\right]\left[\mathrm{BF}_{4}\right](4.0 \mathrm{mg}, 13.8 \mu \mathrm{mol})$ and $\mathrm{Pd}(\mathrm{dba})_{2}(3.1 \mathrm{mg}, 5.4 \mu \mathrm{mol})$ were reacted according to GP3 (Pd-catalyzed Domino Reaction). The mixture was stirred $2 \mathrm{~h}$ at $120{ }^{\circ} \mathrm{C}$. Silica gel column chromatography (EtOAc: $n$-pentane $=1: 50)$ afforded domino product 3d $(31.0 \mathrm{mg}$, $90.9 \mu \mathrm{mol}, 84 \%)$ as yellow oil.

${ }^{1}$ H-NMR (300 MHz, $\left.\mathrm{CDCl}_{3}\right): \delta=1.30(\mathrm{~s}, 9 \mathrm{H}), 1.91$ (dtd, $\left.J=12.2,9.1,5.6 \mathrm{~Hz}, 1 \mathrm{H}\right), 2.81$ (dtd, $J=13.1,8.6,7.9,6.3 \mathrm{~Hz}, 1 \mathrm{H}), 3.10$ (ddd, $J=12.6,9.0,6.1 \mathrm{~Hz}, 1 \mathrm{H}), 3.69$ (ddd, $J=12.5$, 8.8, 5.6 Hz, 1H), 4.70 (dd, $J=9.3,7.2 \mathrm{~Hz}, 1 \mathrm{H}), 6.76-6.92$ (m, 3H), 6.93-7.02 (m, 1H), 7.07$7.25(\mathrm{~m}, 3 \mathrm{H}), 7.74(\mathrm{dd}, J=7.8,1.4 \mathrm{~Hz}, 1 \mathrm{H})$.

${ }^{13}$ C-NMR $\left(75 \mathrm{MHz}, \mathrm{CDCl}_{3}\right): \delta=28.9,35.4,37.2,45.4,80.7,110.6,118.6,120.7,122.9$, $124.9,126.8,129.0,129.2,131.7,143.6,148.2,163.3$.

IR (ATR): $\tilde{v}\left(\mathrm{~cm}^{-1}\right)=2956(\mathrm{~m}), 2873(\mathrm{w}), 1591(\mathrm{~m}), 1488(\mathrm{~s}), 1460(\mathrm{~m}), 1328(\mathrm{~m}), 1220$ (m), $1047(\mathrm{~m})$.

M.p.: $99 \neg 102{ }^{\circ} \mathrm{C}$.

GC-HRMS (EI): $\mathrm{C}_{21} \mathrm{H}_{22} \mathrm{ClNO} \mathrm{m} / z$ calcd. $\left[\mathrm{M}^{+}\right]$: 339.1390 found 339.1411. 


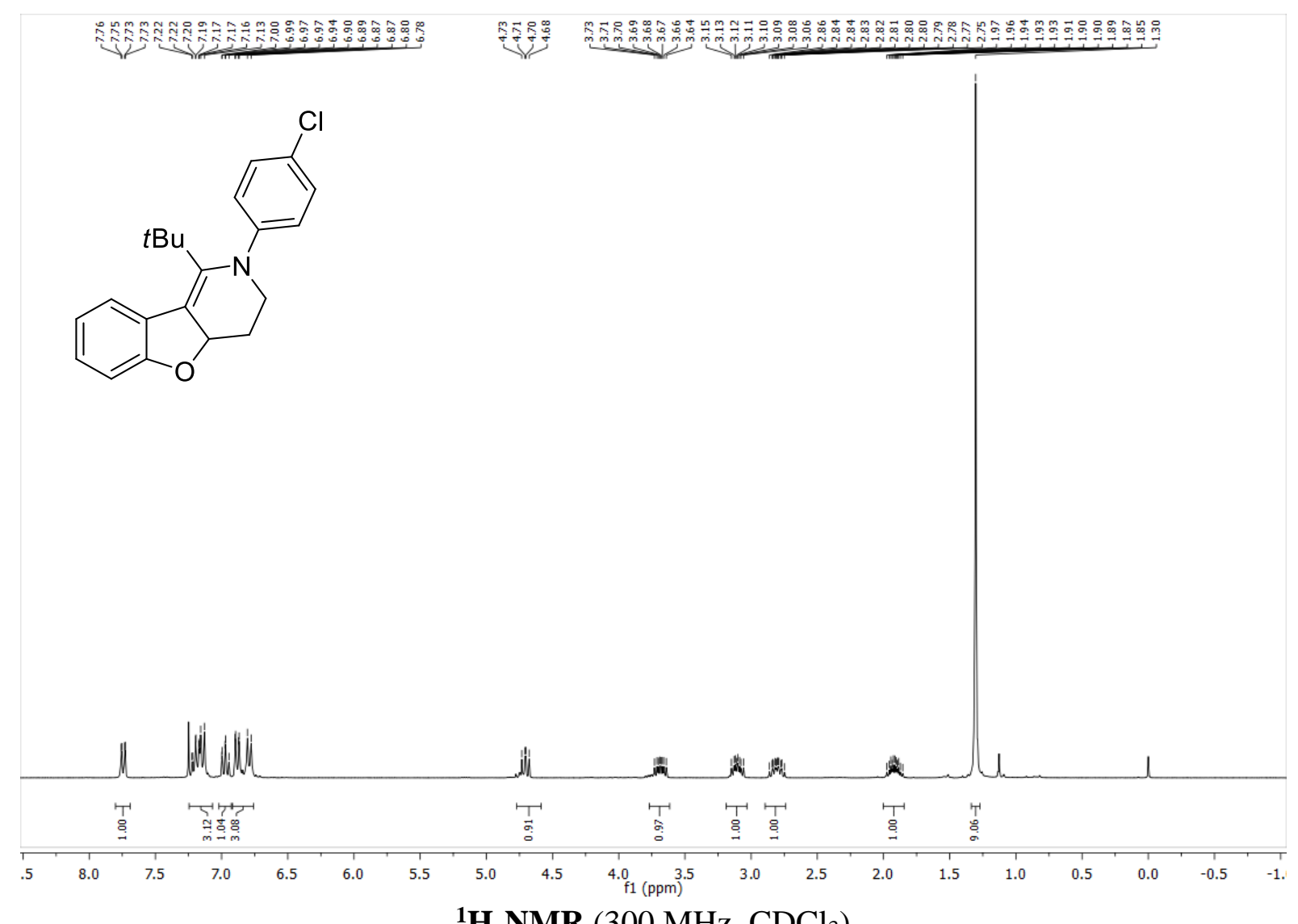

${ }^{1} \mathbf{H}-\mathbf{N M R}\left(300 \mathrm{MHz}, \mathrm{CDCl}_{3}\right.$ )

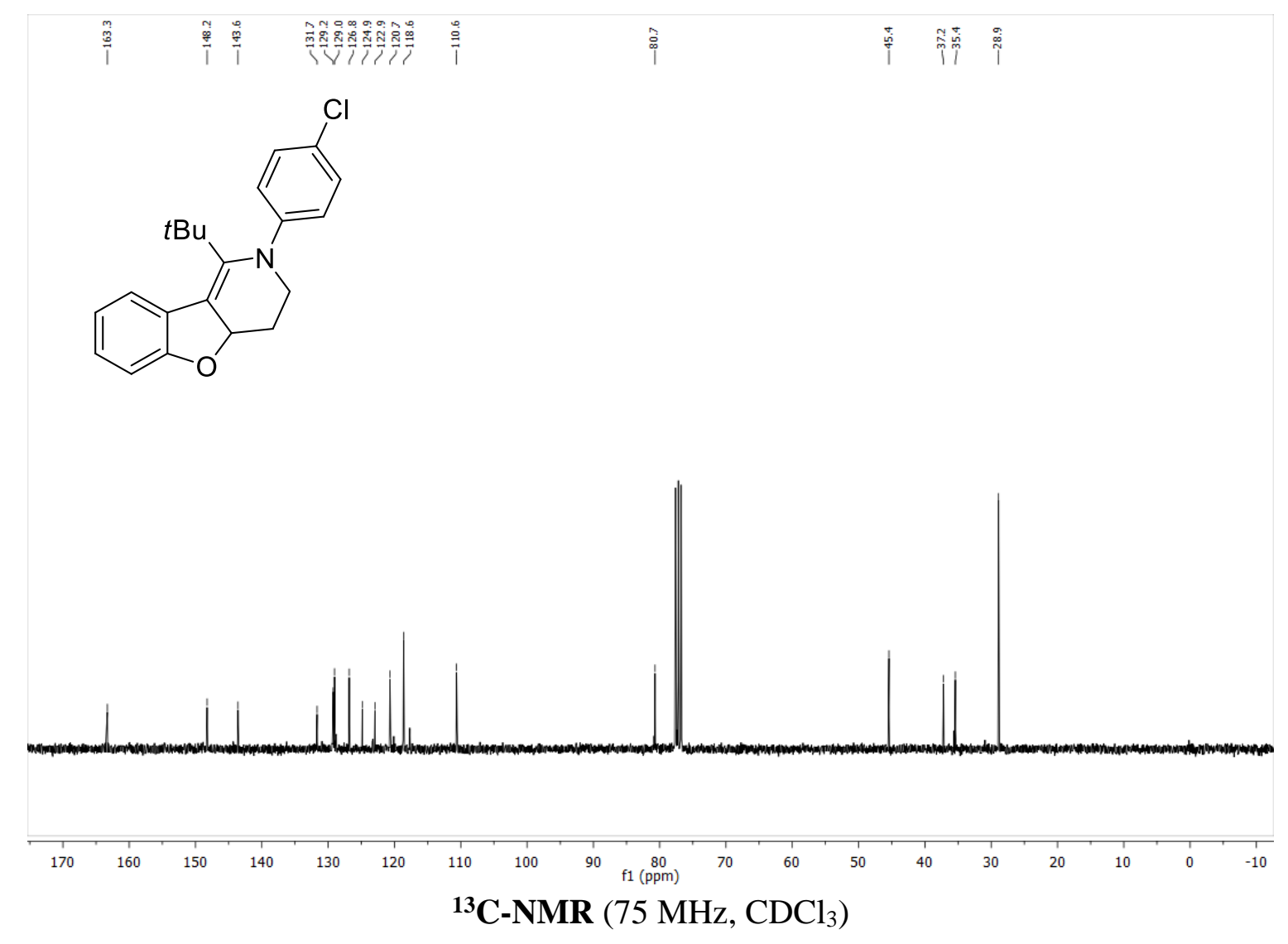




\section{Domino product $3 e$}

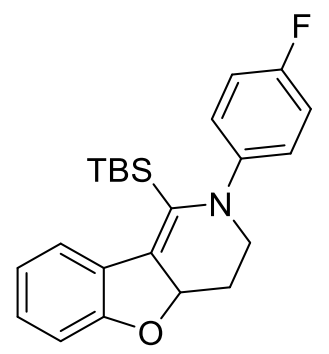

The domino precursor 1e $(58.3 \mathrm{mg}, 126 \mu \mathrm{mol})$, potassium phosphate $(102 \mathrm{mg}, 480 \mu \mathrm{mol})$, $\left[t \mathrm{Bu}_{3} \mathrm{PH}\right]\left[\mathrm{BF}_{4}\right](3.6 \mathrm{mg}, 12.4 \mu \mathrm{mol})$ and $\mathrm{Pd}(\mathrm{dba})_{2}(3.6 \mathrm{mg}, 6.3 \mu \mathrm{mol})$ were reacted according to GP3 (Pd-catalyzed Domino Reaction). The mixture was stirred $2 \mathrm{~h}$ at $120{ }^{\circ} \mathrm{C}$. Silica gel column chromatography (100\% $n$-pentane) afforded domino product $\mathbf{3 e}(12.1 \mathrm{mg}, 31.7 \mu \mathrm{mol}$, $25 \%$ ) as yellow oil.

${ }^{1} \mathrm{H}-\mathrm{NMR}\left(500 \mathrm{MHz}, \mathrm{CDCl}_{3}\right): \delta=-0.24(\mathrm{~s}, 3 \mathrm{H}), 0.42(\mathrm{~s}, 3 \mathrm{H}), 0.86(\mathrm{~s}, 9 \mathrm{H}), 1.91-2.01(\mathrm{~m}$, $1 \mathrm{H}), 2.83-2.93(\mathrm{~m}, 1 \mathrm{H}), 3.34-3.41(\mathrm{~m}, 1 \mathrm{H}), 3.51(\mathrm{dt}, J=11.8,8.5 \mathrm{~Hz}, 1 \mathrm{H}), 4.74$ (dd, $J=10.4,6.1 \mathrm{~Hz}, 1 \mathrm{H}), 6.78(\mathrm{dd}, J=9.0,4.7 \mathrm{~Hz}, 2 \mathrm{H}), 6.86-6.94(\mathrm{~m}, 4 \mathrm{H}), 7.19(\mathrm{ddd}, J=8.5$, 7.3, $1.3 \mathrm{~Hz}, 1 \mathrm{H}), 7.69(\mathrm{dd}, J=8.0,1.3 \mathrm{~Hz}, 1 \mathrm{H})$.

${ }^{13}$ C-NMR $\left(126 \mathrm{MHz}, \mathrm{CDCl}_{3}\right): \delta=-3.5,-3.0,20.0,27.6,35.9,44.4,81.1,110.5,115.4(\mathrm{~d}$, $J=22.1 \mathrm{~Hz}), 118.5(\mathrm{~d}, J=7.6 \mathrm{~Hz}), 120.3,123.8,124.3,129.5,135.8,146.8$ (d, $J=2.2 \mathrm{~Hz})$, 147.2, $157.2(\mathrm{~d}, J=238.4 \mathrm{~Hz}), 163.2$.

${ }^{19} \mathbf{F}-\mathrm{NMR}\left(377 \mathrm{MHz}, \mathrm{CDCl}_{3}\right): \delta=-125.4$.

IR (ATR): $\tilde{v}\left(\mathrm{~cm}^{-1}\right)=2952(\mathrm{~m}), 2934(\mathrm{~m}), 2888(\mathrm{w}), 2859(\mathrm{~m}), 1670(\mathrm{~m}), 1505$ (s), 1460 (m), $1219(\mathrm{~m}), 1038(\mathrm{~m})$.

GC-HRMS (EI): $\mathrm{C}_{23} \mathrm{H}_{28} \mathrm{FNOSi} \mathrm{m} / z$ calcd. $\left[\mathrm{M}^{+}\right]$: 381.1924 found 381.1907. 


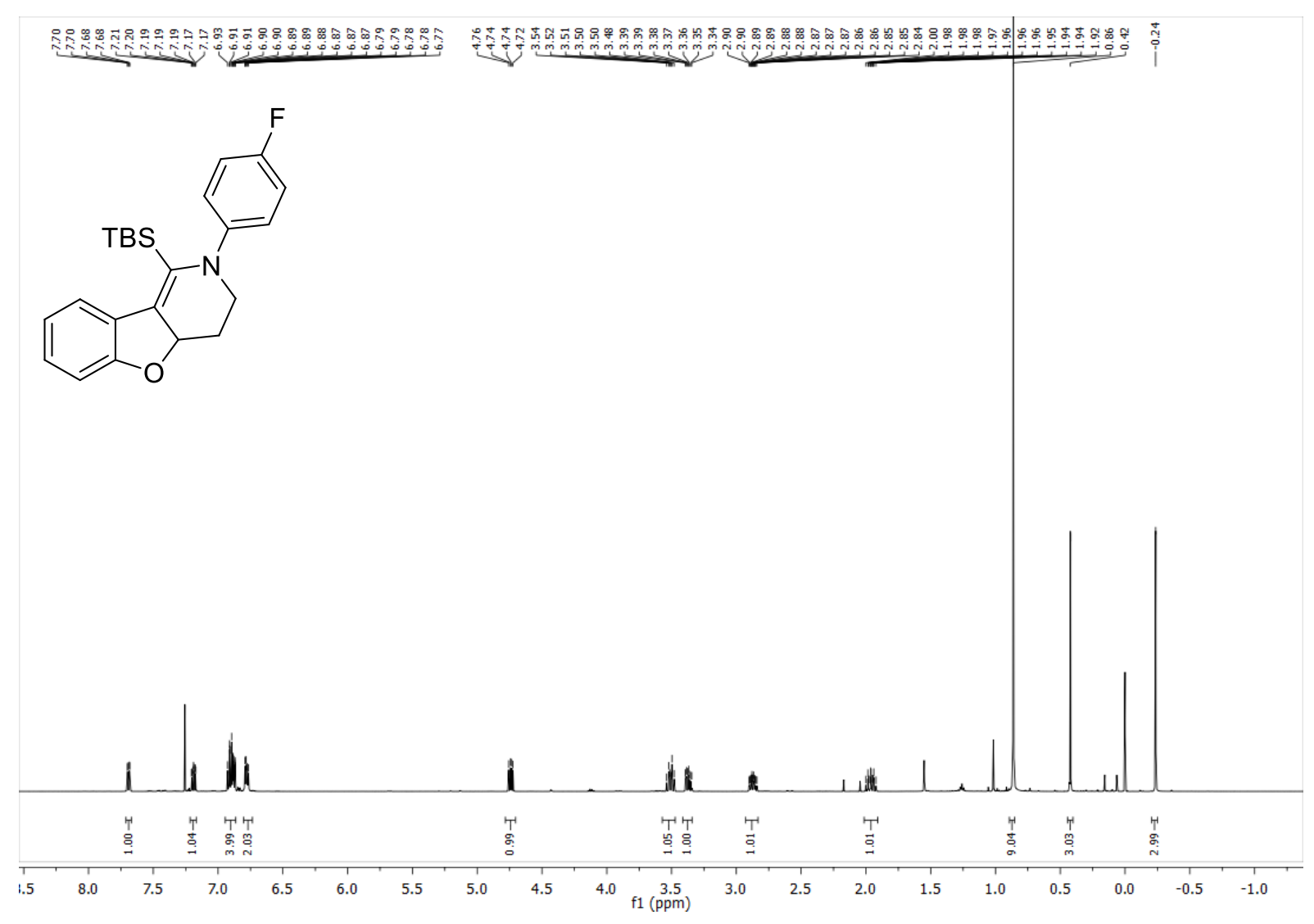

${ }^{1} \mathbf{H}-\mathbf{N M R}\left(500 \mathrm{MHz}, \mathrm{CDCl}_{3}\right)$

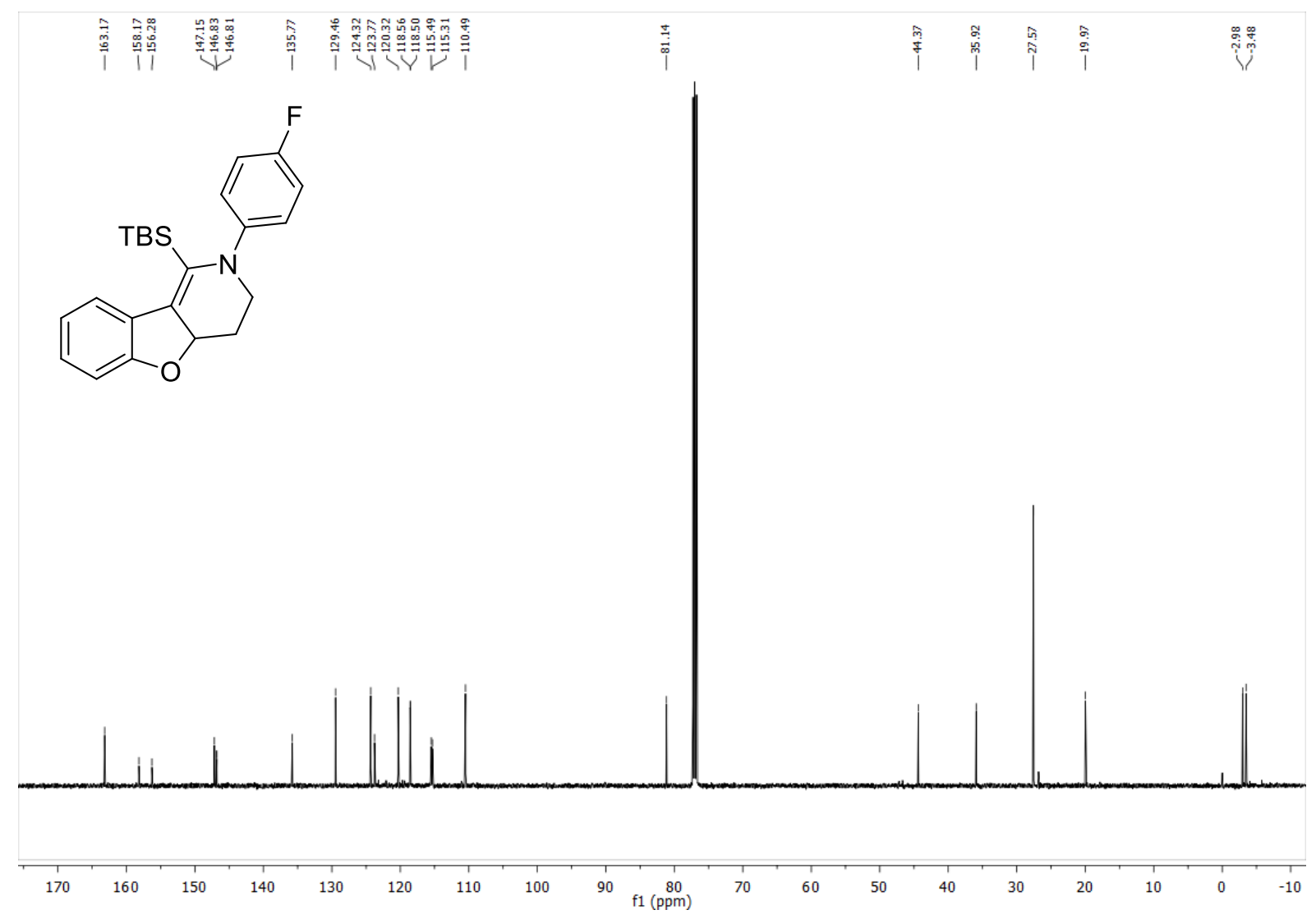

${ }^{13}$ C-NMR $\left(126 \mathrm{MHz}, \mathrm{CDCl}_{3}\right)$ 

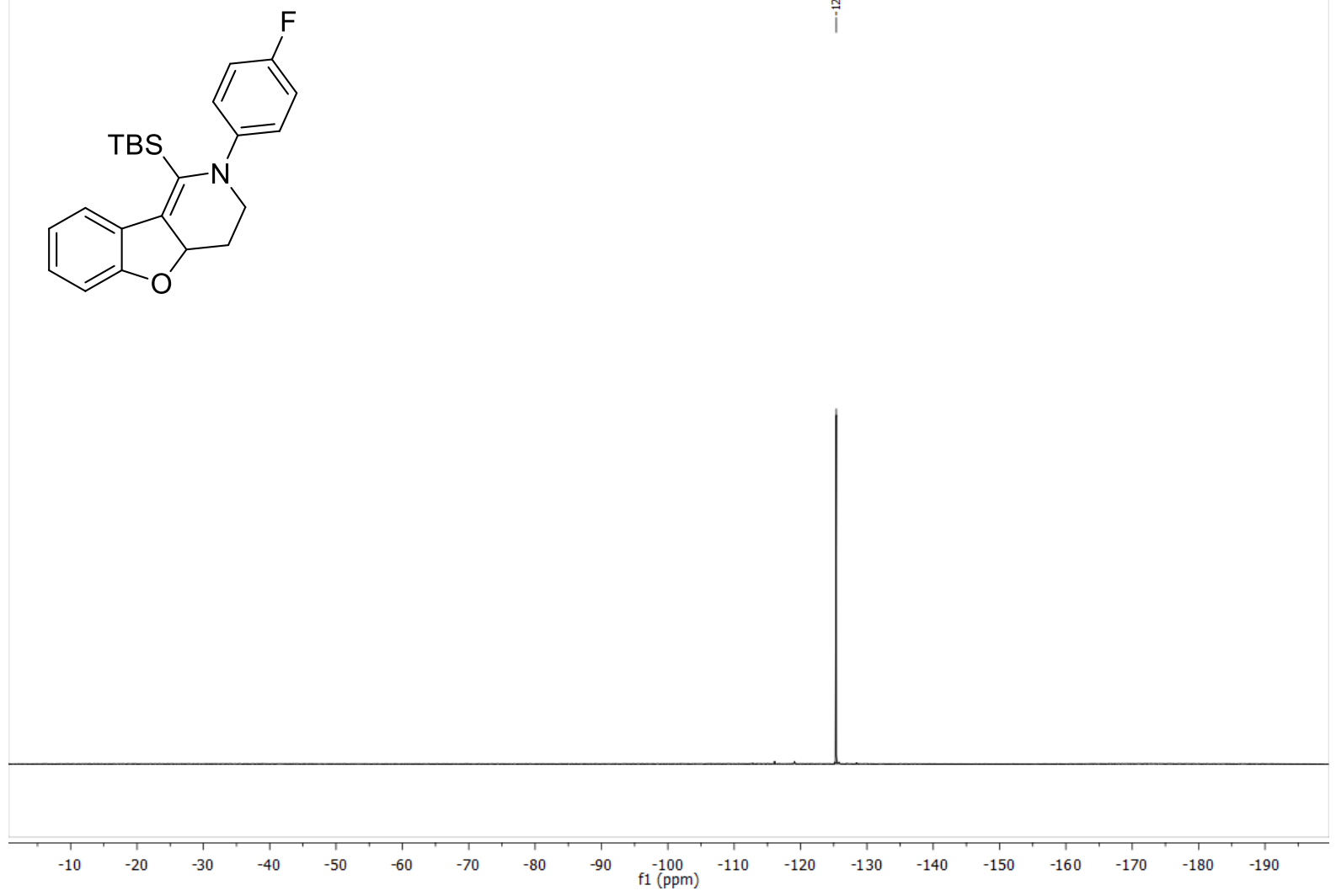

${ }^{19}$ F-NMR (377 MHz, $\left.\mathrm{CDCl}_{3}\right)$ 


\section{Domino product (3f)}<smiles>CC(C)(C)C1=C2c3cc4ccccc4cc3OC2CCN1c1ccccc1</smiles>

The domino precursor 1f $(39.2 \mathrm{mg}, 89.8 \mu \mathrm{mol})$, potassium phosphate $(94 \mathrm{mg}, 443 \mu \mathrm{mol})$, $\left[t \mathrm{Bu}_{3} \mathrm{PH}\right]\left[\mathrm{BF}_{4}\right](2.6 \mathrm{mg}, 9.0 \mu \mathrm{mol})$ and $\mathrm{Pd}(\mathrm{dba})_{2}(2.0 \mathrm{mg}, 3.5 \mu \mathrm{mol})$ were reacted according to GP3 (Pd-catalyzed Domino Reaction). The mixture was stirred $2 \mathrm{~h}$ at $120{ }^{\circ} \mathrm{C}$. Silica gel column chromatography (EtOAc: $n$-pentane=1:20) afforded domino product 3f $(21.6 \mathrm{mg}$, $60.8 \mu \mathrm{mol}, 68 \%$ ) as brown solid.

${ }^{1}$ H-NMR (300 MHz, Acetone- $\left.d_{6}\right): \delta=1.40$ (s, 9H), 1.90-2.01 (m, 1H), 2.81-2.90 (m, 1H), $3.30(\mathrm{ddd}, J=12.4,8.6,6.2 \mathrm{~Hz}, 1 \mathrm{H}), 3.83(\mathrm{ddd}, J=12.5,8.6,5.9 \mathrm{~Hz}, 1 \mathrm{H}), 4.85$ (dd, $J=9.0$, $7.1 \mathrm{~Hz}, 1 \mathrm{H}), 6.89$ (tt, $J=7.3,1.1 \mathrm{~Hz}, 1 \mathrm{H}), 6.94-7.04(\mathrm{~m}, 2 \mathrm{H}), 7.18(\mathrm{q}, J=0.6 \mathrm{~Hz}, 1 \mathrm{H}), 7.25$ $(\mathrm{td}, J=7.4,1.0 \mathrm{~Hz}, 2 \mathrm{H}), 7.32(\mathrm{ddd}, J=8.1,6.9,1.4 \mathrm{~Hz}, 1 \mathrm{H}), 7.41(\mathrm{ddd}, J=8.3,6.8,1.4 \mathrm{~Hz}$, $1 \mathrm{H}), 7.73(\mathrm{ddd}, J=8.1,1.3,0.6 \mathrm{~Hz}, 1 \mathrm{H}), 7.92(\mathrm{ddd}, J=8.1,1.4,0.7 \mathrm{~Hz}, 1 \mathrm{H}), 8.23(\mathrm{~s}, 1 \mathrm{H})$.

${ }^{13}$ C-NMR (126 MHz, Acetone- $\left.d_{6}\right) \delta=28.8,36.2,38.1,46.9,81.8,104.6,119.1,121.4,124.2$, $125.8,126.6,127.0,127.2,129.5,130.0,130.4,135.3,148.4$ 150.5, 162.1.

IR (ATR): $\tilde{v}\left(\mathrm{~cm}^{-1}\right)=2953(\mathrm{w}), 2928(\mathrm{w}), 2869$ (w), 1592 (m), 1490 (m), 1444 (m), 1233 (m), $1219(\mathrm{~m}), 1155(\mathrm{~m})$.

M.p.: $174 \neg 177{ }^{\circ} \mathrm{C}$.

GC-HRMS (EI): $\mathrm{C}_{25} \mathrm{H}_{25} \mathrm{NO} \mathrm{m} / \mathrm{z}$ calcd. $\left[\mathrm{M}^{+}\right]$: 355.1936 found 355.1949. 

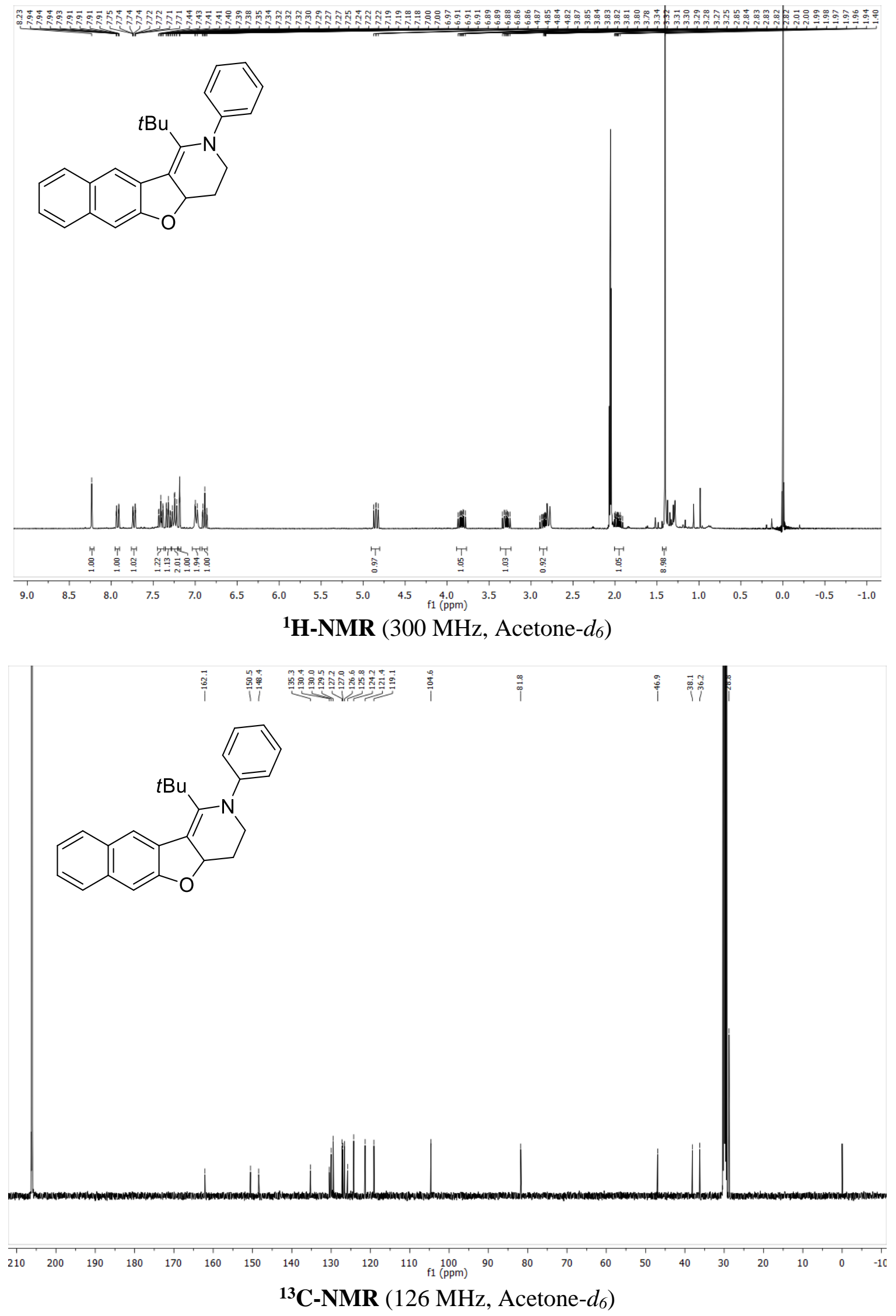


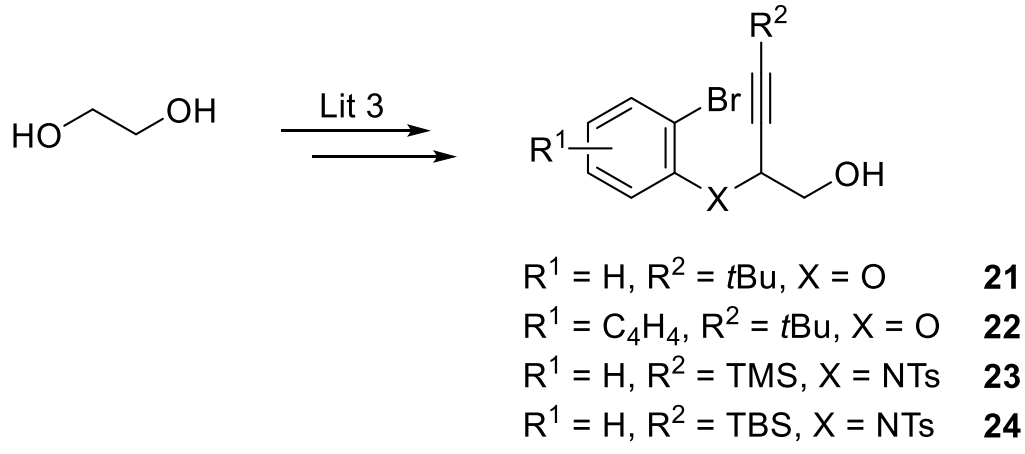

1) $\mathrm{Tf}_{2} \mathrm{O}$

2) amine

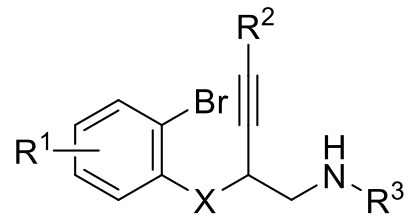

$$
\begin{aligned}
& \mathrm{R}^{1}=\mathrm{H}, \mathrm{R}^{2}=t \mathrm{Bu}, \mathrm{R}^{3}=\mathrm{Ph}, \mathrm{X}=\mathrm{O} \\
& \text { 2a }(84 \%) \\
& \mathrm{R}^{1}=\mathrm{H}, \mathrm{R}^{2}=t \mathrm{Bu}, \mathrm{R}^{3}=3,5-(\mathrm{OMe})_{2}-\mathrm{Ph}, \mathrm{X}=\mathrm{O} \\
& \text { 2b }(40 \%) \\
& \mathrm{R}^{1}=\mathrm{H}, \mathrm{R}^{2}=t \mathrm{Bu}, \mathrm{R}^{3}=4-\mathrm{Cl}-\mathrm{Ph}, \mathrm{X}=\mathrm{O} \\
& \text { 2c }(38 \%) \\
& R^{1}=\text { naphthyl, } R^{2}=t B u, R^{3}=P h, X=0 \\
& \text { 2d }(80 \%) \\
& \mathrm{R}^{1}=\mathrm{H}, \mathrm{R}^{2}=t \mathrm{Bu}, \mathrm{R}^{3}=2,4,6-\mathrm{Me}_{3}-\mathrm{Ph}, \mathrm{X}=\mathrm{O} \\
& \text { 2e }(67 \%) \\
& R^{1}=H, R^{2}=T M S, R^{3}=2,4,6-M_{3}-P h, X=N T s \\
& 2 f(42 \%) \\
& \mathrm{R}^{1}=\mathrm{H}, \mathrm{R}^{2}=\mathrm{TMS}, \mathrm{R}^{3}=\mathrm{Ph}, \mathrm{X}=\mathrm{NTS} \\
& \text { 2g }(44 \%) \\
& R^{1}=H, R^{2}=T B S, R^{3}=P h, X=N T s \\
& \text { 2h }(73 \%) \\
& \mathrm{R}^{1}=\mathrm{H}, \mathrm{R}^{2}=t \mathrm{Bu}, \mathrm{R}^{3}=i \mathrm{Pr}, \mathrm{X}=\mathrm{O} \\
& \text { 2i }(65 \%) \\
& R^{1}=H, R^{2}=t B u, R^{3}=B n, X=O \\
& \text { 2j }(79 \%)
\end{aligned}
$$




\section{$N$-(2-(2-Bromophenoxy)-5,5-dimethylhex-3-yn-1-yl)aniline (2a)}<smiles>CC(C)(C)C#CC(CNc1ccccc1)Oc1ccccc1Br</smiles>

Alcohol $2^{3}(1.18 \mathrm{~g}, 3.96 \mathrm{mmol})$ was reacted with DIPEA $(900 \mu \mathrm{L}, 5.17 \mathrm{mmol}), \mathrm{Tf}_{2} \mathrm{O}$ $(750 \mu \mathrm{L}, 4.57 \mathrm{mmol})$ and aniline $(600 \mu \mathrm{L}, 6.57 \mathrm{mmol})$ according to GP4 (Synthesis of Amine (B)). The reaction mixture was stirred at ambient temperature overnight. Silica gel column chromatography (EtOAc: $n$-pentane = 1:50) afforded compound 2a $(1.24 \mathrm{~g}, 3.33 \mathrm{mmol}, 84 \%)$ as colorless oil.

${ }^{1}$ H-NMR (400 MHz, $\left.\mathrm{CDCl}_{3}\right): \delta=1.19$ (s, 9H), 3.61-3.67 (m, 2H), $4.38(\mathrm{~s}, 1 \mathrm{H}), 4.83$ (dd, $J=7.4,4.9 \mathrm{~Hz}, 1 \mathrm{H}), 6.68-6.75(\mathrm{~m}, 3 \mathrm{H}), 6.87(\mathrm{ddd}, J=8.0,7.1,1.7 \mathrm{~Hz}, 1 \mathrm{H}), 7.15-7.24(\mathrm{~m}$, $4 \mathrm{H}), 7.53(\mathrm{dd}, J=7.9,1.6 \mathrm{~Hz}, 1 \mathrm{H})$.

${ }^{13}$ C-NMR $\left(101 \mathrm{MHz}, \mathrm{CDCl}_{3}\right) \delta=27.5,30.7,48.9,69.2,74.7,97.7,113.3,113.6,116.8$, $118.1,123.0,128.2,129.3,133.2,147.4,154.3$.

IR (ATR): $\tilde{v}\left(\mathrm{~cm}^{-1}\right)=3411(\mathrm{w}), 3054(\mathrm{w}), 3023(\mathrm{w}), 2969(\mathrm{~m}), 2928(\mathrm{~m}), 2901(\mathrm{~m}), 2867$ (m), $1603(\mathrm{~m}), 1475(\mathrm{~s}), 1262(\mathrm{~m}), 1241(\mathrm{~m}), 1030(\mathrm{~m})$.

GC-HRMS (EI): $\mathrm{C}_{20} \mathrm{H}_{22} \mathrm{BrNO} \mathrm{m} / \mathrm{z}$ calcd. $\left[\mathrm{M}^{+}\right]$: 371.0885 found 371.0895 . 


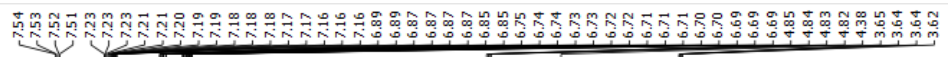<smiles>CC(C)(C)C#CC(CNc1ccccc1)Oc1ccccc1Br</smiles>
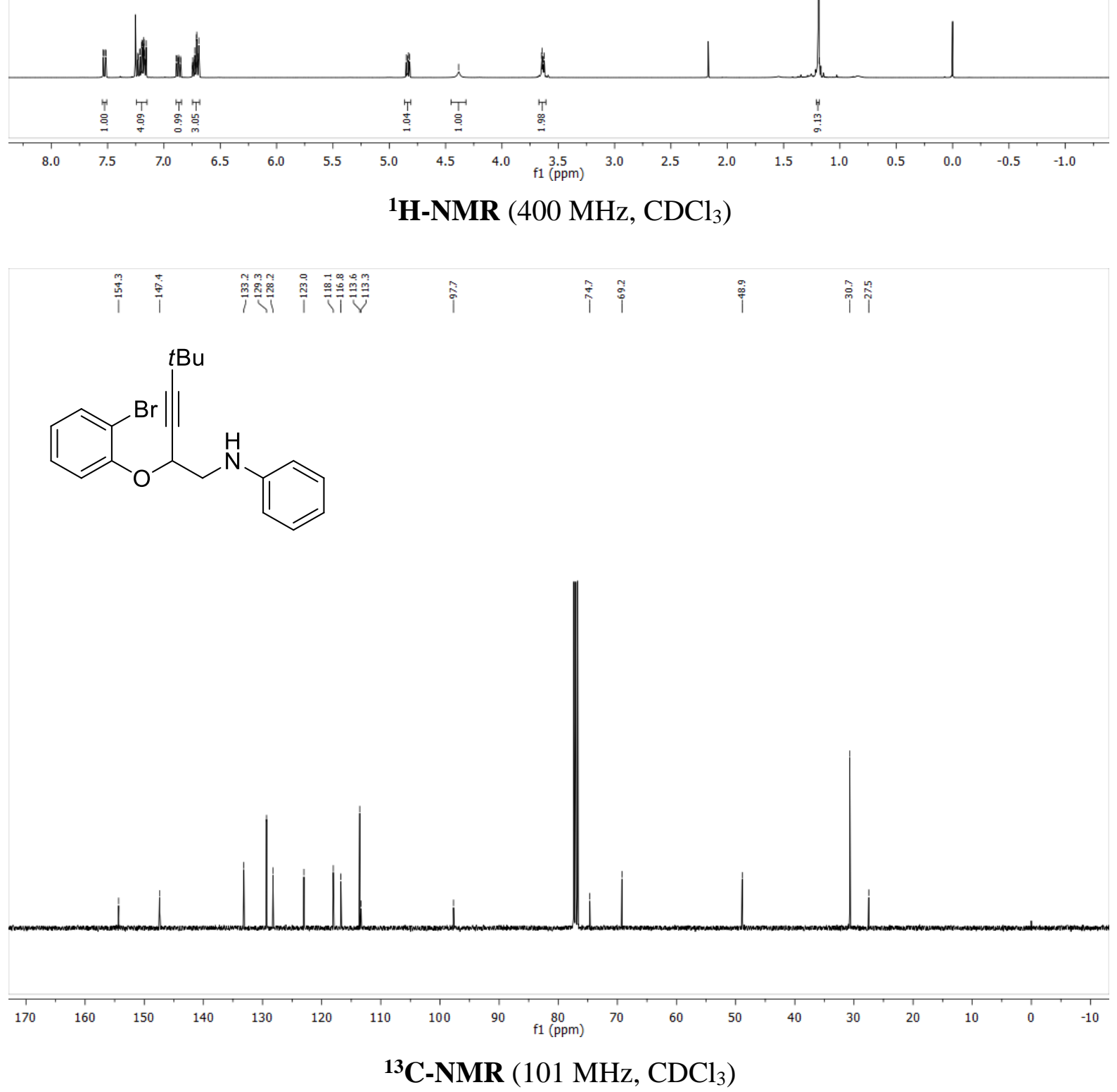

S39 
$N$-(2-(2-Bromophenoxy)-5,5-dimethylhex-3-yn-1-yl)-3,5-dimethoxyaniline (2b)<smiles>COc1cc(NCC(C#CC(C)(C)C)Oc2ccccc2Br)cc(OC)c1</smiles>

Alcohol $21^{3}(225 \mathrm{mg}, 757 \mu \mathrm{mol})$ was reacted with DIPEA $(180 \mu \mathrm{L}, 1.03 \mathrm{mmol}), \mathrm{Tf}_{2} \mathrm{O}$ $(140 \mu \mathrm{L}, 854 \mu \mathrm{mol})$ and 3,5-dimethoxyaniline $(226 \mathrm{mg}, 1.47 \mathrm{mmol})$ according to GP4 (Synthesis of Amine (B)). The reaction mixture was stirred at ambient temperature overnight. Silica gel column chromatography (EtOAc: $n$-pentane $=1: 15)$ afforded compound $\mathbf{2 b}$ (130 mg, $300 \mu \mathrm{mol}, 40 \%)$ as colorless oil.

${ }^{1}$ H-NMR (400 MHz, $\left.\mathrm{CDCl}_{3}\right): \delta=1.19$ (s, 9H), 3.56-3.63 (m, 2H), 3.74 (s, 6H), $4.41(\mathrm{~s}, 1 \mathrm{H})$, $4.83(\mathrm{dd}, J=7.1,5.2 \mathrm{~Hz}, 1 \mathrm{H}), 5.85-5.92(\mathrm{~m}, 3 \mathrm{H}), 6.84-6.90(\mathrm{~m}, 1 \mathrm{H}), 7.15-7.25(\mathrm{~m}, 2 \mathrm{H})$, $7.52(\mathrm{dd}, J=7.9,1.6 \mathrm{~Hz}, 1 \mathrm{H})$.

${ }^{13}$ C-NMR (101 MHz, $\left.\mathrm{CDCl}_{3}\right): \delta=27.5,30.7,48.9,55.2,69.1,74.6,90.3,92.5,97.7,113.3$, $116.8,123.0,128.3,133.1,149.3,154.3,161.8$.

IR (ATR): $\tilde{v}\left(\mathrm{~cm}^{-1}\right)=3407(\mathrm{w}), 2967$ (m), 2905 (m), $2867(\mathrm{w}), 2841(\mathrm{w}), 1611(\mathrm{~s}), 1474(\mathrm{~m})$, $1238(\mathrm{~m}), 1203 \mathrm{~s}), 1153(\mathrm{~s})$.

ESI-HRMS: $\mathrm{C}_{22} \mathrm{H}_{26} \mathrm{BrNO}_{3} \mathrm{~m} / z$ calcd. $\left[\mathrm{M}+\mathrm{H}^{+}\right]$: 432.1169 found 432.1174 . 


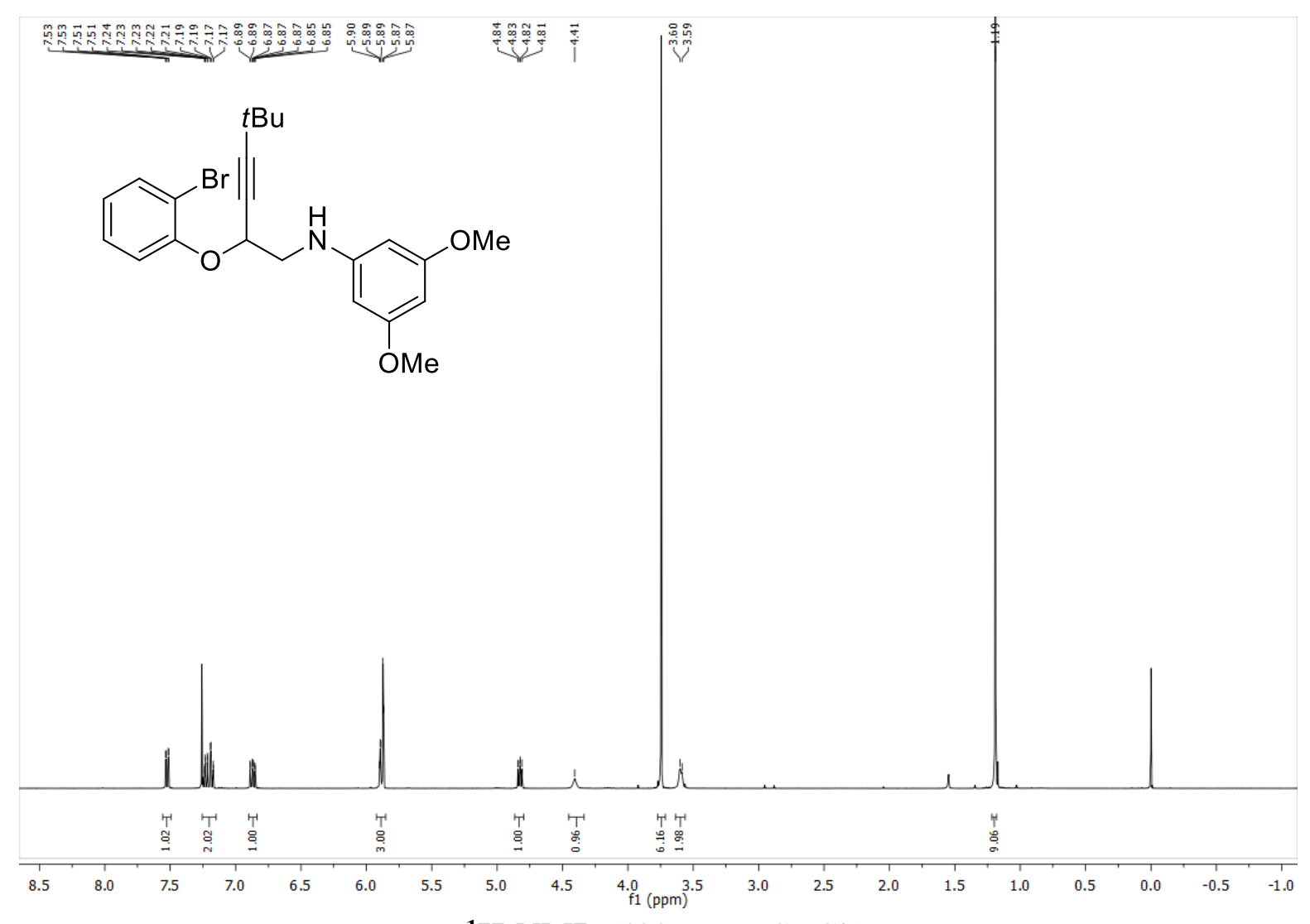

${ }^{1} \mathbf{H}$-NMR (400 MHz, $\mathrm{CDCl}_{3}$ )

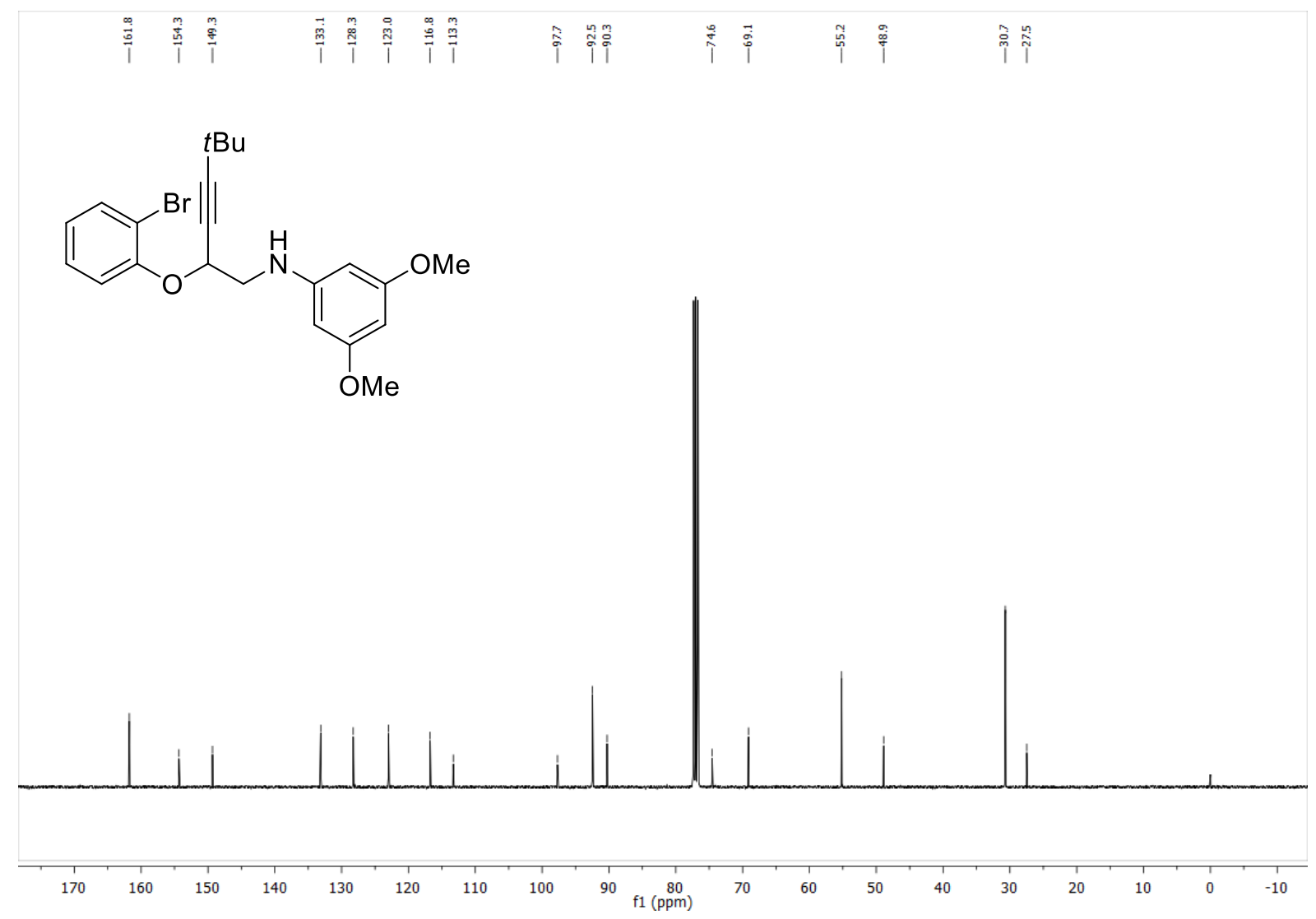

${ }^{13}$ C-NMR (101 MHz, $\left.\mathrm{CDCl}_{3}\right)$ 


\section{$N$-(2-(2-Bromophenoxy)-5,5-dimethylhex-3-yn-1-yl)-4-chloroaniline (2c)}<smiles>CC(C)(C)C#CC(CNc1ccc(Cl)cc1)Oc1ccccc1Br</smiles>

Alcohol $\mathbf{2 1}^{3}$ (148 mg, $\left.498 \mu \mathrm{mol}\right)$ was reacted with DIPEA $(140 \mu \mathrm{L}, 804 \mu \mathrm{mol}), \mathrm{Tf}_{2} \mathrm{O}$ $(100 \mu \mathrm{L}, 610 \mu \mathrm{mol})$ and 4-chloroaniline $(128 \mathrm{mg}, 1.00 \mathrm{mmol})$ according to GP4 (Synthesis of Amine (B)). The reaction mixture was stirred at ambient temperature overnight. Silica gel column chromatography (EtOAc: $n$-pentane $=1: 25)$ afforded compound 2c $(77.8 \mathrm{mg}$, $191 \mu \mathrm{mol}, 38 \%$ ) as yellow oil.

${ }^{1}$ H-NMR (500 MHz, $\left.\mathrm{CDCl}_{3}\right): \delta=1.18(\mathrm{~s}, 9 \mathrm{H}), 3.57-3.63(\mathrm{~m}, 2 \mathrm{H}), 4.41(\mathrm{~s}, 1 \mathrm{H}), 4.81$ (dd, $J=6.8,5.4 \mathrm{~Hz}, 1 \mathrm{H}), 6.60-6.64(\mathrm{~m}, 2 \mathrm{H}), 6.86-6.92$ (m, 1H), 7.09-7.14 (m, 2H), 7.18 (dd, $J=8.3,1.6 \mathrm{~Hz}, 1 \mathrm{H}), 7.22-7.26(\mathrm{~m}, 1 \mathrm{H}), 7.53(\mathrm{dd}, J=7.9,1.5 \mathrm{~Hz}, 1 \mathrm{H})$.

${ }^{13} \mathrm{C}-\mathrm{NMR}\left(126 \mathrm{MHz}, \mathrm{CDCl}_{3}\right): \delta=27.5,30.7,49.0,69.1,74.4,97.9,113.3,114.6,116.8$, $122.6,123.2,128.3,129.1,133.2,146.0,154.2$.

IR (ATR): $\tilde{v}\left(\mathrm{~cm}^{-1}\right)=3405(\mathrm{w}), 2969(\mathrm{~m}), 2927(\mathrm{w}), 2902(\mathrm{w}), 2868(\mathrm{w}), 1598(\mathrm{~m}), 1502(\mathrm{~s})$, 1474 (s), $1238(\mathrm{~s}), 1028(\mathrm{~m})$.

ESI-HRMS: $\mathrm{C}_{20} \mathrm{H}_{21} \mathrm{BrClNO} \mathrm{m} / \mathrm{z}$ calcd. $\left[\mathrm{M}+\mathrm{H}^{+}\right]$: 406.0568 found 406.0571. 


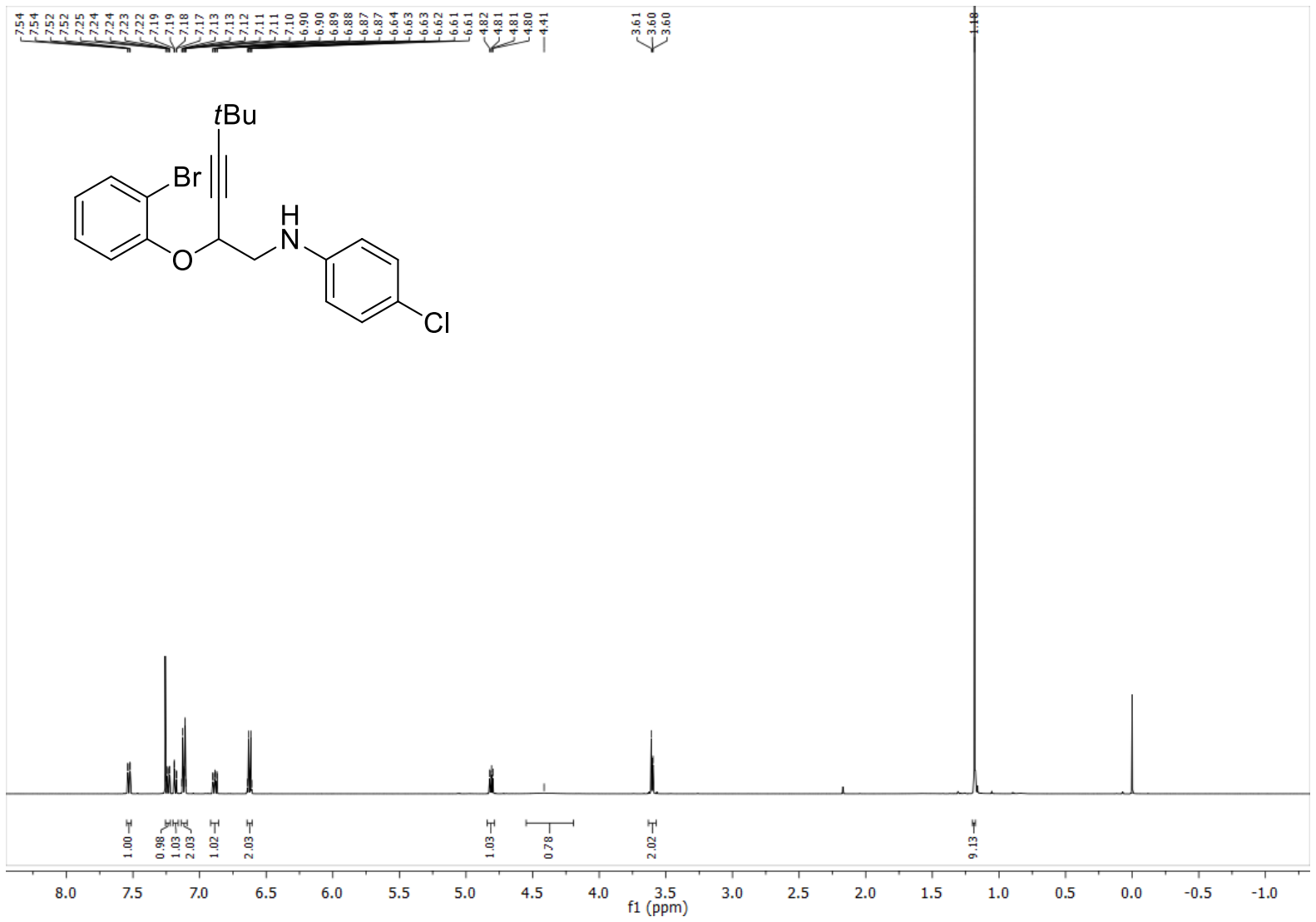

${ }^{1} \mathbf{H}-\mathbf{N M R}\left(500 \mathrm{MHz}, \mathrm{CDCl}_{3}\right)$

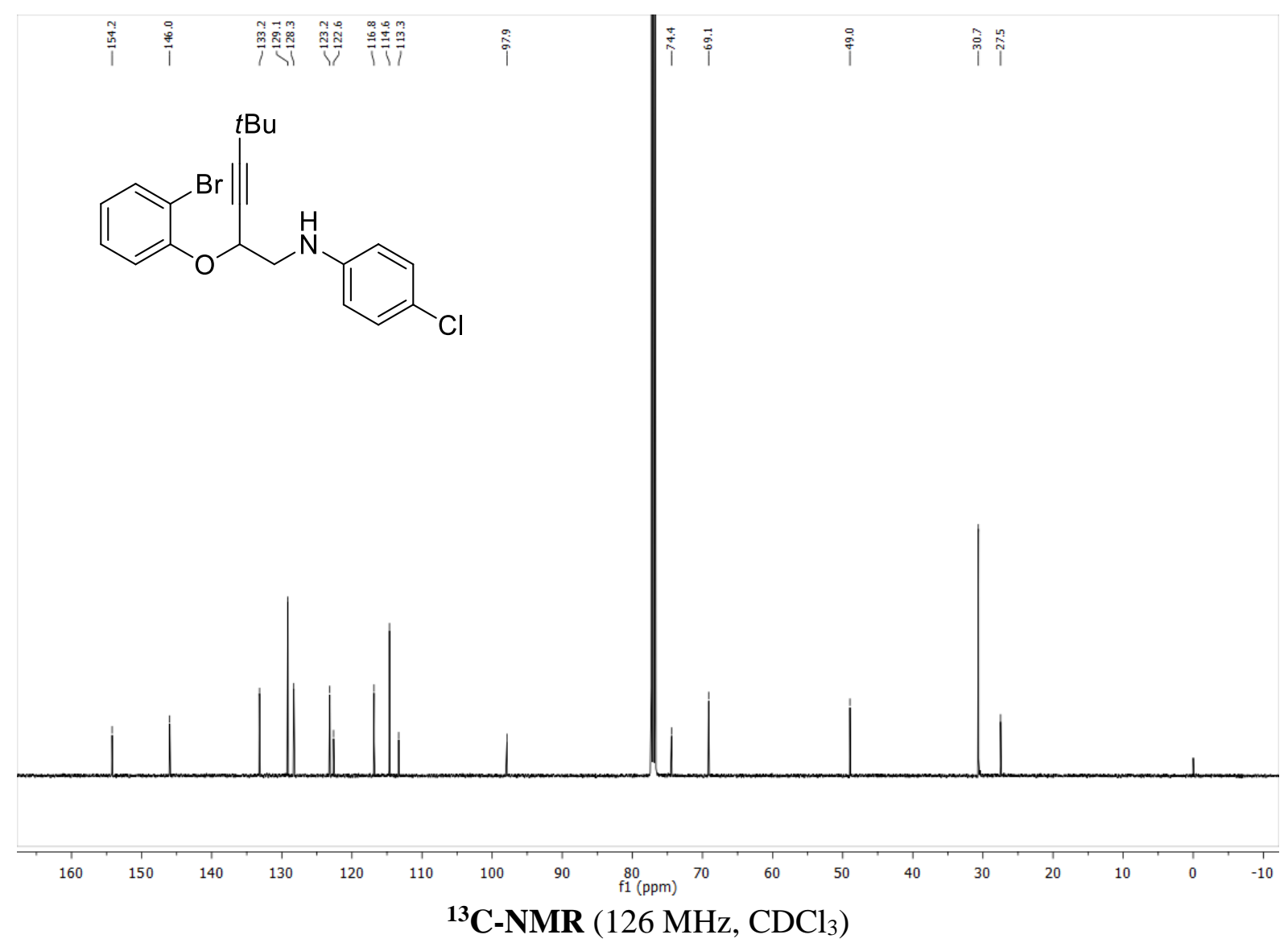




\section{$N$-(2-((3-Bromonaphthalen-2-yl)oxy)-5,5-dimethylhex-3-yn-1-yl)aniline (2d)}<smiles>CC(C)(C)C#CC(CNc1ccccc1)Oc1cc2ccccc2cc1Br</smiles>

Alcohol $\mathbf{2 2}^{3}(173 \mathrm{mg}, 499 \mu \mathrm{mol})$ was reacted with DIPEA $(130 \mu \mathrm{L}, 746 \mu \mathrm{mol}), \mathrm{Tf}_{2} \mathrm{O}$ $(130 \mu \mathrm{L}, 793 \mu \mathrm{mol})$ and aniline $(92 \mu \mathrm{L}, 1.01 \mathrm{mmol})$ according to GP4 (Synthesis of Amine (B)). The reaction mixture was stirred at ambient temperature overnight. Silica gel column chromatography (EtOAc: $n$-pentane = 1:25) afforded compound 2d (149 mg, $401 \mu \mathrm{mol}, 80 \%)$ as yellow oil.

${ }^{1} \mathbf{H}$-NMR (300 MHz, $\left.\mathrm{CDCl}_{3}\right): \delta=1.20(\mathrm{~s}, 9 \mathrm{H}), 3.61-3.78(\mathrm{~m}, 2 \mathrm{H}), 4.40(\mathrm{t}, J=6.7 \mathrm{~Hz}, 1 \mathrm{H})$, $4.96(\mathrm{dd}, J=7.1,5.3 \mathrm{~Hz}, 1 \mathrm{H}), 6.68-6.77(\mathrm{~m}, 3 \mathrm{H}), 7.13-7.23(\mathrm{~m}, 2 \mathrm{H}), 7.36-7.51(\mathrm{~m}, 3 \mathrm{H})$, 7.65-7.72 (m, 2H), $8.06(\mathrm{~s}, 1 \mathrm{H})$.

${ }^{13}$ C-NMR $\left(75 \mathrm{MHz}, \mathrm{CDCl}_{3}\right): \delta=27.5,30.7,48.8,69.0,74.6,98.0,111.3,113.6,114.2$, $118.1,124.8,126.6,126.7,126.8,129.3,129.9,132.0,133.3,147.3,151.6$.

IR (ATR): $\tilde{v}\left(\mathrm{~cm}^{-1}\right)=3405(\mathrm{w}), 3055(\mathrm{w}), 2969(\mathrm{~m}), 2928(\mathrm{~m}), 2902(\mathrm{~m}), 2868(\mathrm{w}), 1599(\mathrm{~s})$, 1504 (s), 1454 (s), 1245 (s), 1216 (s), 1024 (m).

ESI-HRMS: $\mathrm{C}_{24} \mathrm{H}_{24} \mathrm{BrNO} \mathrm{m} / z$ calcd. $\left[\mathrm{M}+\mathrm{Na}^{+}\right]$: 444.0933 found 444.0939. 


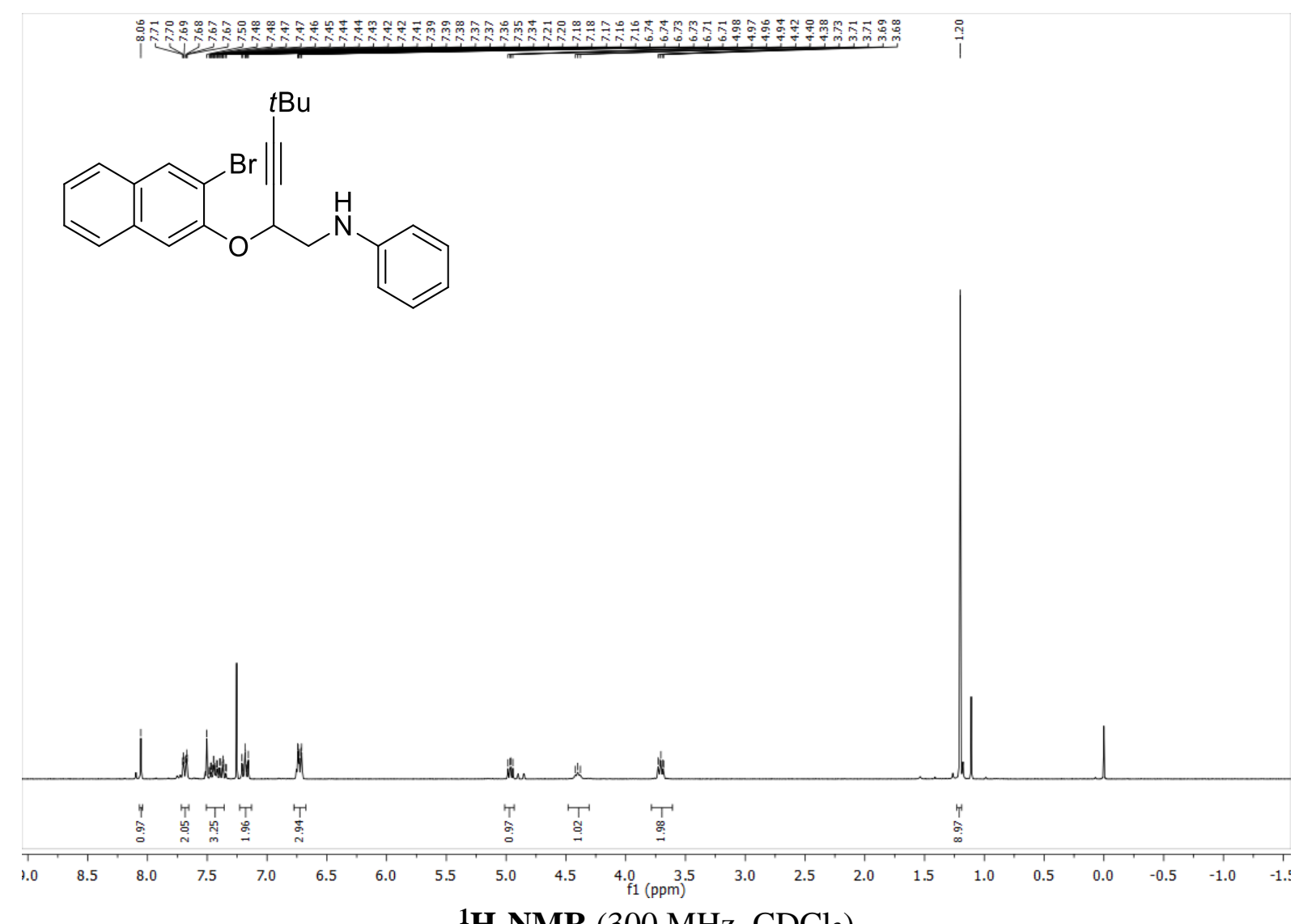

${ }^{1} \mathbf{H}-\mathbf{N M R}\left(300 \mathrm{MHz}, \mathrm{CDCl}_{3}\right)$

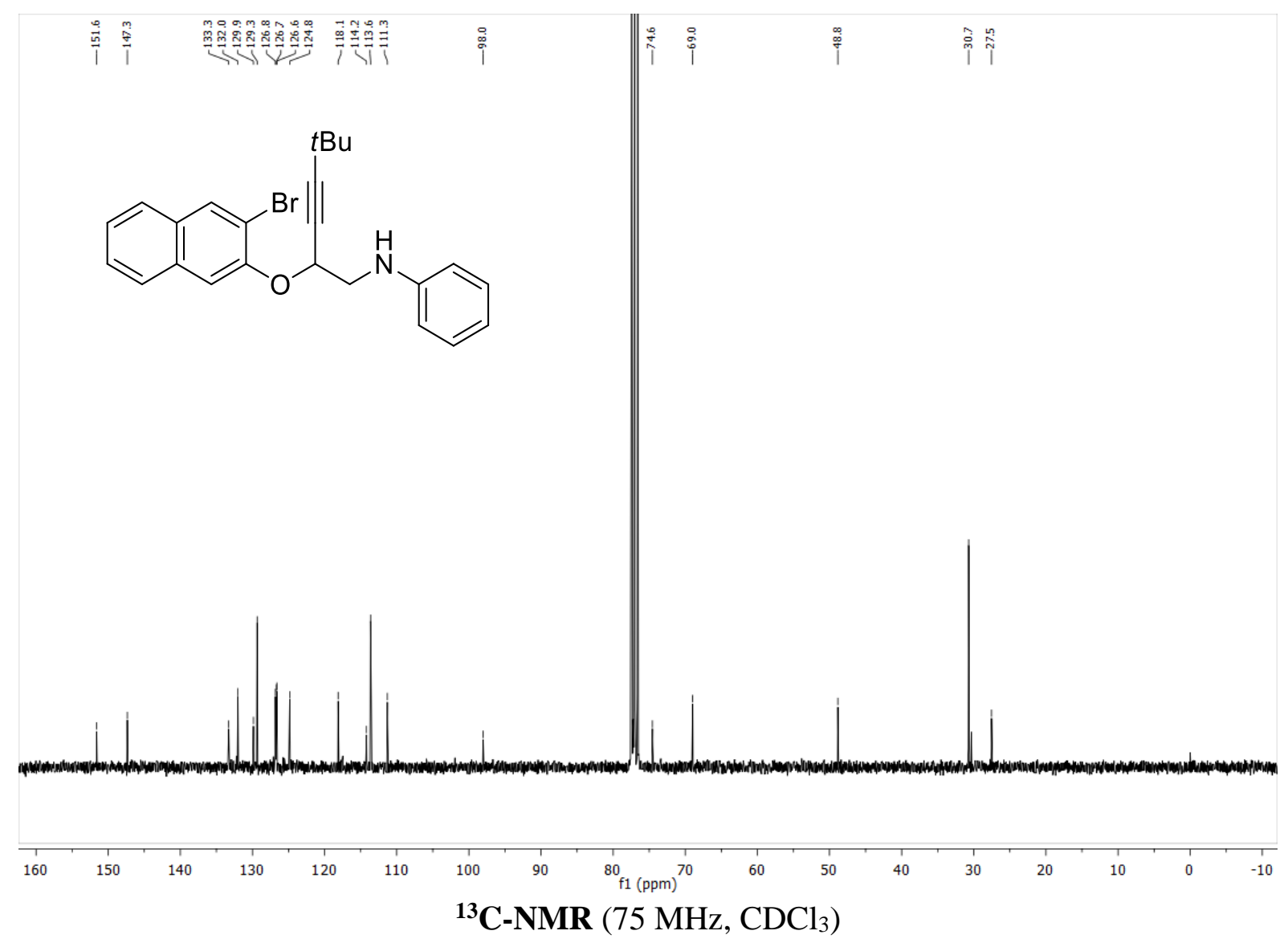




\section{$N$-(2-(2-Bromophenoxy)-5,5-dimethylhex-3-yn-1-yl)-2,4,6-trimethylaniline (2e)}<smiles>Cc1cc(C)c(NCC(C#CC(C)(C)C)Oc2ccccc2Br)c(C)c1</smiles>

Alcohol $\mathbf{2 1}^{3}(147 \mathrm{mg}, 495 \mu \mathrm{mol})$ was reacted with DIPEA $(120 \mu \mathrm{L}, 689 \mu \mathrm{mol}), \mathrm{Tf}_{2} \mathrm{O}$ $(100 \mu \mathrm{L}, 610 \mu \mathrm{mol})$ and 2,4,6-trimethylaniline $(150 \mu \mathrm{L}, 1.07 \mathrm{mmol})$ according to GP4 (Synthesis of Amine (B)). The reaction mixture was stirred at ambient temperature overnight. Silica gel column chromatography (EtOAc: $n$-pentane $=1: 50)$ afforded compound $2 \mathbf{e}$ (138 mg, $333 \mu \mathrm{mol}, 67 \%)$ as yellow oil.

${ }^{1}$ H-NMR (500 MHz, $\mathrm{CDCl}_{3}$ ): $\delta=1.16$ (s, 9H), 2.22 (s, 3H), 2.28 (s, 6H), 3.34 (dd, $J=13.1$, $3.9 \mathrm{~Hz}, 1 \mathrm{H}), 3.47(\mathrm{dd}, J=13.2,8.0 \mathrm{~Hz}, 1 \mathrm{H}), 3.80(\mathrm{~s}, 1 \mathrm{H}), 4.77(\mathrm{dd}, J=8.0,3.7 \mathrm{~Hz}, 1 \mathrm{H}), 6.81$ (s, 2H), $6.87(\mathrm{td}, J=7.6,1.5 \mathrm{~Hz}, 1 \mathrm{H}), 7.16(\mathrm{dd}, J=8.4,1.4 \mathrm{~Hz}, 1 \mathrm{H}), 7.23-7.26(\mathrm{~m}, 1 \mathrm{H}), 7.55$ (dd, $J=7.8,1.6 \mathrm{~Hz}, 1 \mathrm{H})$.

${ }^{13}$ C-NMR (126 MHz, $\left.\mathrm{CDCl}_{3}\right): \delta=18.2,20.6,27.4,30.7,53.0,69.4,74.6,97.4,112.8,115.6$, $122.5,128.2,129.5,130.2,133.2,154.2$.

IR (ATR): $\tilde{v}\left(\mathrm{~cm}^{-1}\right)=$ 3379(w), 2969 (m), $2923(\mathrm{~m}), 2865(\mathrm{w}), 1477$ (s), $1280(\mathrm{~m}), 1238(\mathrm{~s})$, $1028(\mathrm{~m})$.

ESI-HRMS: $\mathrm{C}_{23} \mathrm{H}_{28} \mathrm{BrNO} \mathrm{m} / \mathrm{z}$ calcd. $\left[\mathrm{M}+\mathrm{H}^{+}\right]$: 414.1427 found 414.1434 . 

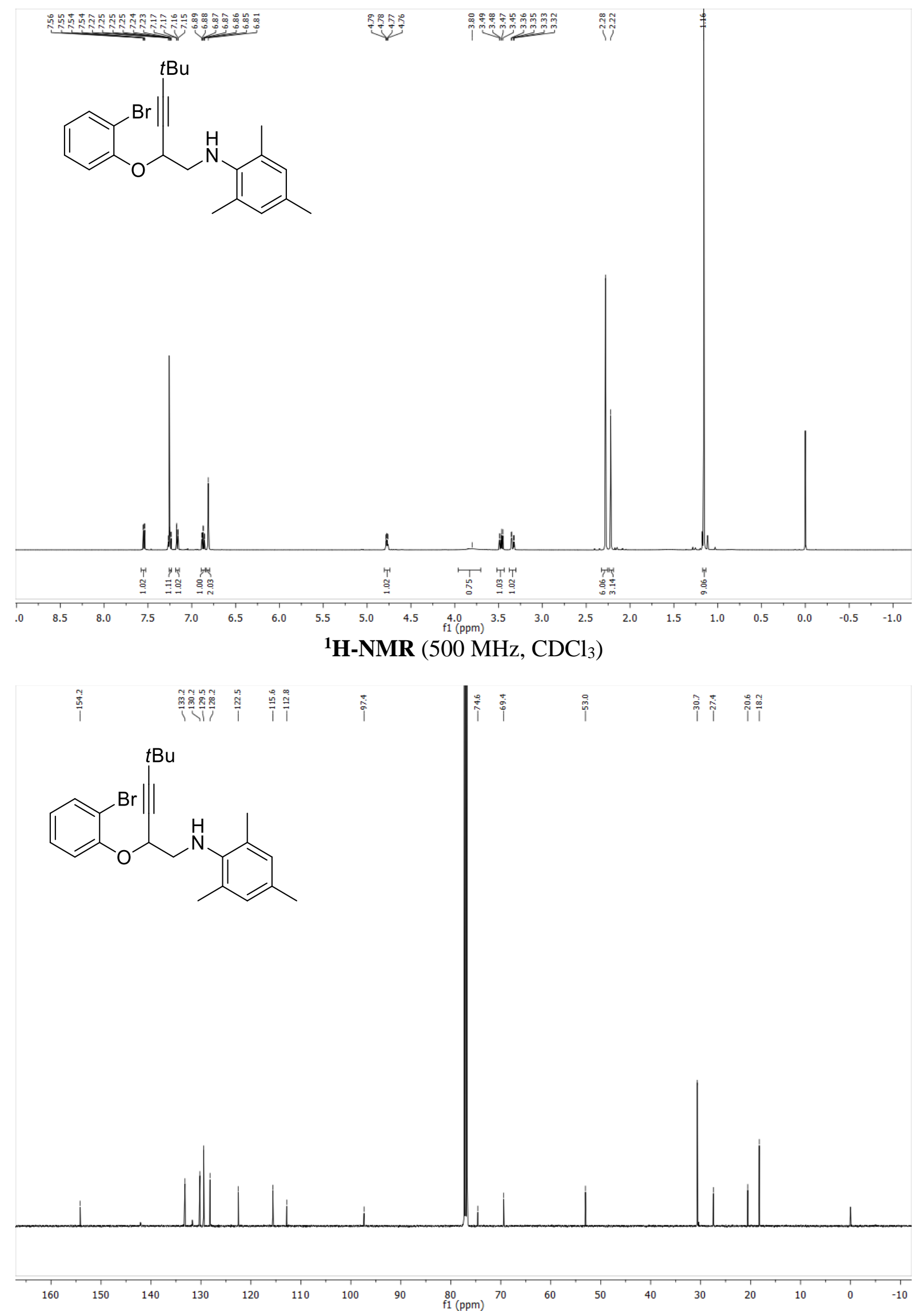

${ }^{13}$ C-NMR (126 MHz, $\mathrm{CDCl}_{3}$ ) 
$N$-(2-Bromophenyl)- $N$-(1-(mesitylamino)-4-(trimethylsilyl)but-3-yn-2-yl)-4-methylbenzenesulfonamide (2f)<smiles>Cc1cc(C)c(NCC(C#CC(C)(C)C)N(C)c2ccccc2Br)c(C)c1</smiles>

Alcohol $\mathbf{2 3}^{3}(232 \mathrm{mg}, 497 \mu \mathrm{mol})$ was reacted with DIPEA $(270 \mu \mathrm{L}, 1.55 \mathrm{mmol}), \mathrm{Tf}_{2} \mathrm{O}$ $(140 \mu \mathrm{L}, 854 \mu \mathrm{mol})$ and 2,4,6-trimethylaniline $(142 \mu \mathrm{L}, 1.01 \mathrm{mmol})$ according to GP4 (Synthesis of Amine (B)). The reaction mixture was stirred at ambient temperature overnight. Silica gel column chromatography (EtOAc: $n$-pentane $=1: 15)$ afforded compound $\mathbf{2 f}$ (122 mg, $209 \mu \mathrm{mol}, 42 \%)$ as yellow solid.

${ }^{1} \mathbf{H}-\mathrm{NMR}\left(600 \mathrm{MHz}, \mathrm{CDCl}_{3}\right): \delta=0.02(\mathrm{~s}, 9 \mathrm{H}), 2.22(\mathrm{~s}, 3 \mathrm{H}), 2.24(\mathrm{~s}, 6 \mathrm{H}), 2.45(\mathrm{~s}, 3 \mathrm{H}), 2.74$ $(\mathrm{dd}, J=12.2,8.8 \mathrm{~Hz}, 1 \mathrm{H}), 3.57(\mathrm{dd}, J=12.3,5.6 \mathrm{~Hz}, 1 \mathrm{H}), 5.15(\mathrm{dd}, J=8.7,5.6 \mathrm{~Hz}, 1 \mathrm{H})$, $6.78(\mathrm{~d}, J=12.4 \mathrm{~Hz}, 2 \mathrm{H}), 7.15-7.21(\mathrm{~m}, 1 \mathrm{H}), 7.20-7.27(\mathrm{~m}, 1 \mathrm{H}), 7.26-7.31(\mathrm{~m}, 2 \mathrm{H}), 7.66(\mathrm{~d}$, $J=8.2 \mathrm{~Hz}, 2 \mathrm{H}), 7.70(\mathrm{dd}, J=8.0,1.6 \mathrm{~Hz}, 1 \mathrm{H})$.

${ }^{13} \mathbf{C}-\mathbf{N M R}\left(151 \mathrm{MHz}, \mathrm{CDCl}_{3}\right): \delta=-0.5,17.7,18.9,20.7,21.8,51.2,53.4,93.5,102.2,122.0$, 127.3, 129.0, 129.0, 129.4, 129.6, 129.7, 130.5, 131.5, 132.0, 134.2, 136.0, 136.1, 144.0.

IR (ATR): $\tilde{v}\left(\mathrm{~cm}^{-1}\right)=3396(\mathrm{w}), 2959$ (w), $2920(\mathrm{w}), 2858(\mathrm{w}), 1480$ (m), 1358 (m), 1250 (m), $1165(\mathrm{~s}), 1068(\mathrm{~m})$.

M.p.: $122 \neg 125^{\circ} \mathrm{C}$.

ESI-HRMS: $\mathrm{C}_{29} \mathrm{H}_{35} \mathrm{BrN}_{2} \mathrm{O}_{2} \mathrm{SSi} / z$ calcd. $\left[\mathrm{M}+\mathrm{H}^{+}\right]$: 583.1445 found 583.1449. 


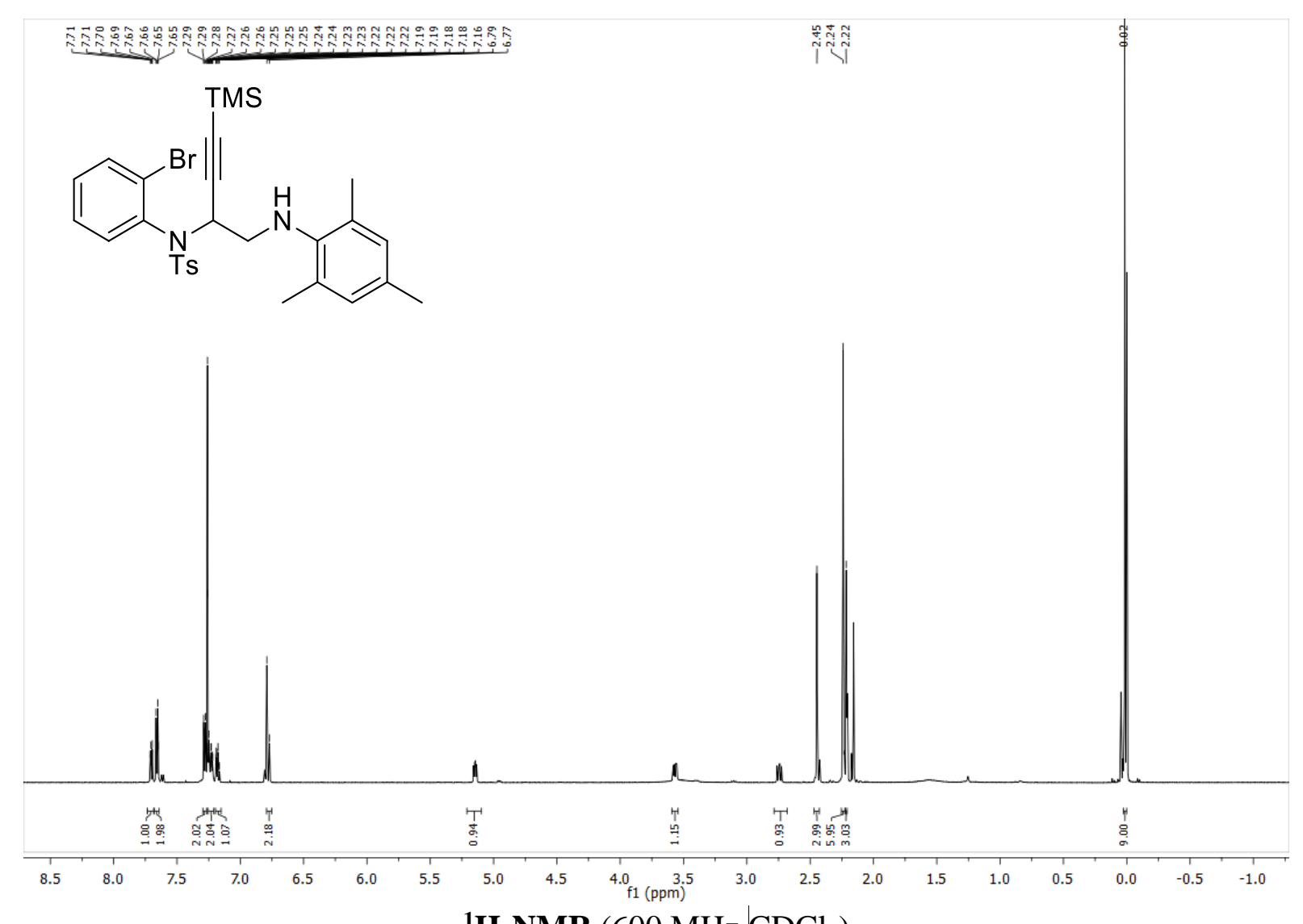

${ }^{\mathbf{1}} \mathbf{H}$-NMR (600 MHz, $\left.\mathrm{CDCl}_{3}\right)$
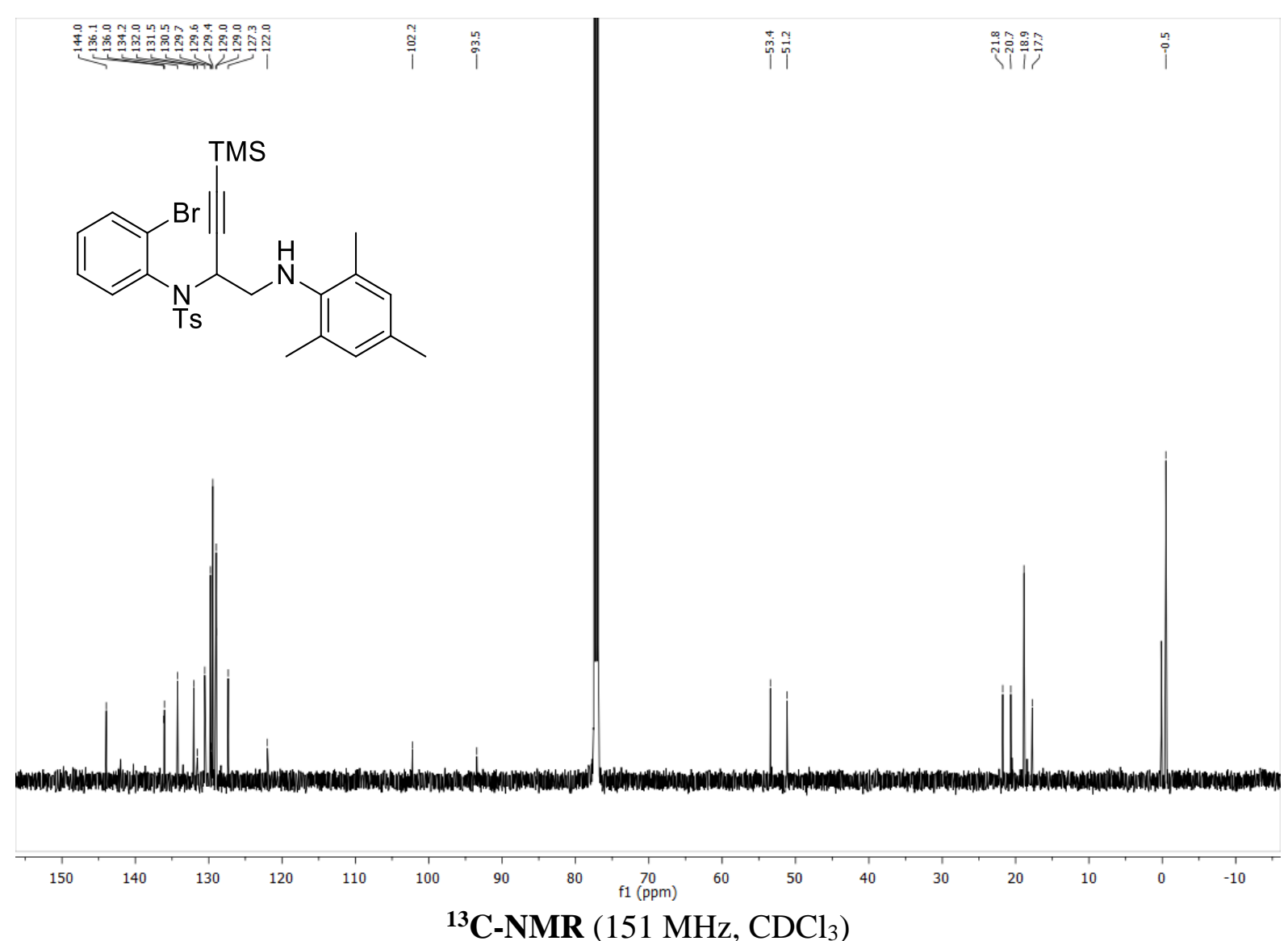

${ }^{13}$ C-NMR (151 MHz, $\left.\mathrm{CDCl}_{3}\right)$ 

benzenesulfonamide $(2 \mathrm{~g})$<smiles>CC#CC(CNc1ccccc1)N(C)c1ccccc1Br</smiles>

Alcohol $\mathbf{2 3}^{3}$ (103 mg, $\left.221 \mu \mathrm{mol}\right)$ was reacted with DIPEA $(130 \mu \mathrm{L}, 746 \mu \mathrm{mol}), \mathrm{Tf}_{2} \mathrm{O}(50 \mu \mathrm{L}$, $305 \mu \mathrm{mol}$ ) and aniline (42 $\mu \mathrm{L}, 460 \mu \mathrm{mol})$ according to GP4 (Synthesis of Amine (B)). The reaction mixture was stirred at ambient temperature overnight. Silica gel column chromatography (EtOAc: $n$-pentane =1:10) afforded compound $\mathbf{2 g}(52.7 \mathrm{mg}, 97.3 \mu \mathrm{mol}$, $44 \%$ ) as yellow solid.

${ }^{1} \mathbf{H}-\mathrm{NMR}\left(300 \mathrm{MHz}, \mathrm{CDCl}_{3}\right): \delta=-0.03(\mathrm{~s}, 9 \mathrm{H}), 2.40(\mathrm{~s}, 3 \mathrm{H}), 3.11(\mathrm{dd}, J=13.5,6.6 \mathrm{~Hz}, 1 \mathrm{H})$, $3.44(\mathrm{dd}, J=13.5,7.5 \mathrm{~Hz}, 1 \mathrm{H}), 4.17(\mathrm{~s}, 1 \mathrm{H}), 5.20(\mathrm{dd}, J=7.4,6.5 \mathrm{~Hz}, 1 \mathrm{H}), 6.57-6.64(\mathrm{~m}$, 2H), 6.67-6.75 (m, 1H), 7.12-7.18 (m, 3H), $7.25(\mathrm{~d}, J=1.9 \mathrm{~Hz}, 2 \mathrm{H}), 7.58-7.64(\mathrm{~m}, 2 \mathrm{H})$, $7.68(\mathrm{dd}, J=7.7,1.8 \mathrm{~Hz}, 1 \mathrm{H})$.

${ }^{13} \mathrm{C}-\mathrm{NMR}\left(75 \mathrm{MHz}, \mathrm{CDCl}_{3}\right): \delta=-0.5,21.7,47.0,52.4,93.5,101.4,113.6,118.2,127.3$, $128.7,128.8,129.5,129.5,130.6,132.5,134.2,135.6,136.0,144.1,147.0$.

IR (ATR): $\tilde{v}\left(\mathrm{~cm}^{-1}\right)=3416(\mathrm{w}), 3056$ (w), 3026 (w), 2958 (w), 2901 (w), 2866 (w), 1602 (m), 1506 (m),1 1470 (m), 1354 (m), 1252 (m), 1164 (s), 1071 (m).

M.p.: $51 \neg 54{ }^{\circ} \mathrm{C}$.

ESI-HRMS: $\mathrm{C}_{26} \mathrm{H}_{29} \mathrm{BrN}_{2} \mathrm{O}_{2} \mathrm{SSi} / z$ calcd. $\left[\mathrm{M}+\mathrm{H}^{+}\right]$: 541.0975 found 541.0982 . 


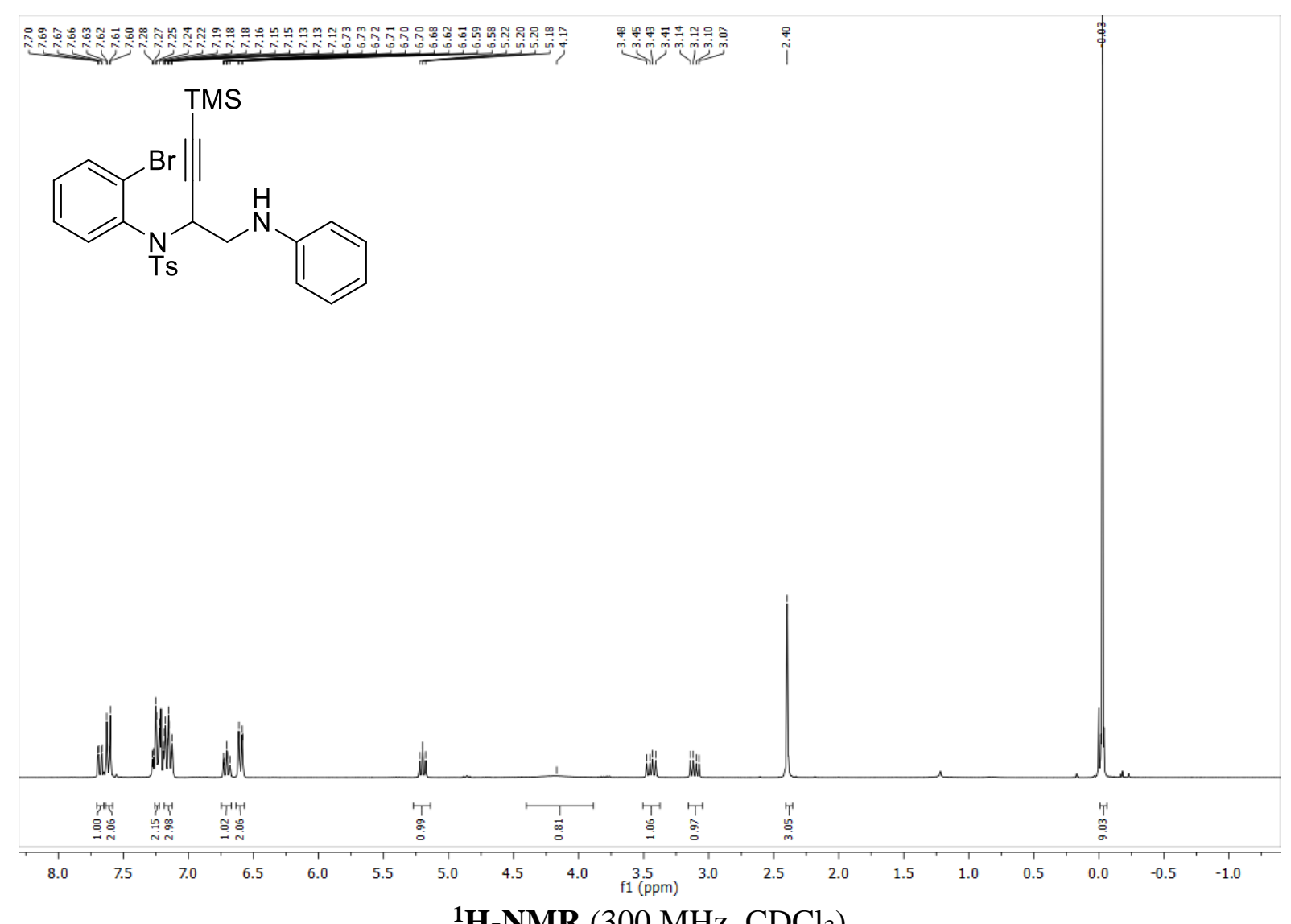

${ }^{1} \mathbf{H}-\mathbf{N M R}\left(300 \mathrm{MHz}, \mathrm{CDCl}_{3}\right)$

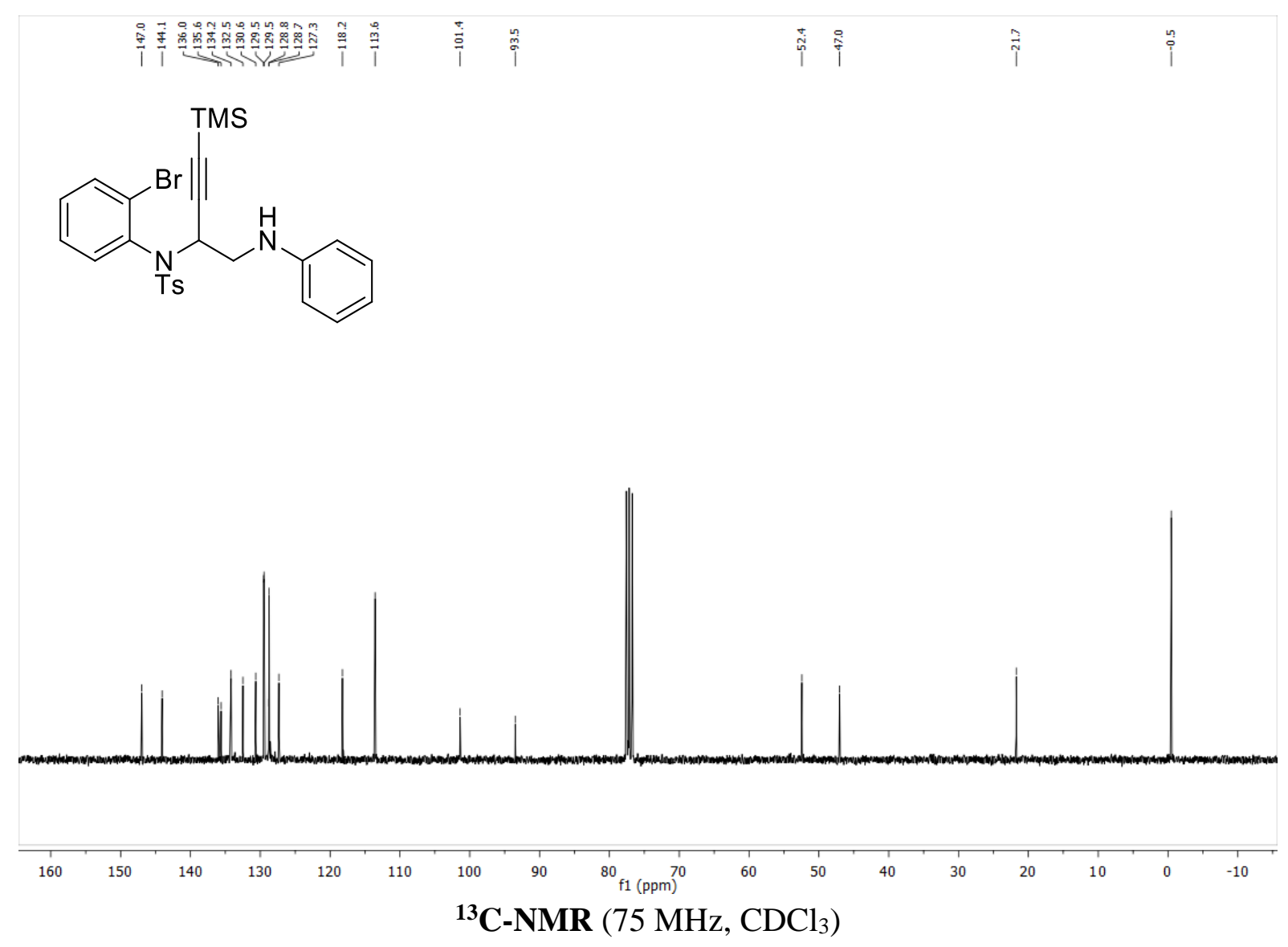


$N$-(2-Bromophenyl)- $N$-(4-(tert-butyldimethylsilyl)-1-(phenylamino)but-3-yn-2-yl)-4methylbenzenesulfonamide $(2 \mathrm{~h})$

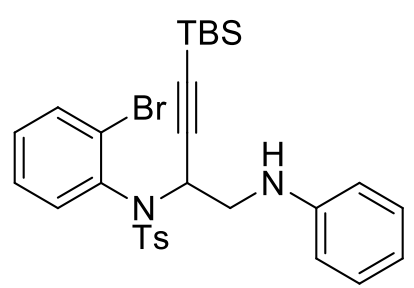

Alcohol 24 $\mathbf{4}^{4}(1.02 \mathrm{~g}, 2.01 \mathrm{mmol})$ was reacted with DIPEA $(700 \mu \mathrm{L}, 4.12 \mathrm{mmol}), \mathrm{Tf}_{2} \mathrm{O}$ $(470 \mu \mathrm{L}, 2.80 \mathrm{mmol})$ and aniline $(420 \mu \mathrm{L}, 4.60 \mathrm{mmol})$ according to GP4 (Synthesis of Amine (B)). The reaction mixture was stirred at ambient temperature overnight. Silica gel column chromatography (EtOAc: $n$-pentane $=1: 20)$ afforded compound $\mathbf{2 h}(855 \mathrm{mg}, 1.47 \mathrm{mmol}$, $73 \%$ ) as yellow oil.

${ }^{1} \mathbf{H}-\mathrm{NMR}\left(300 \mathrm{MHz}, \mathrm{CDCl}_{3}\right): \delta=-0.04(\mathrm{~d}, J=3.3 \mathrm{~Hz}, 6 \mathrm{H}), 0.80(\mathrm{~s}, 9 \mathrm{H}), 2.44(\mathrm{~s}, 3 \mathrm{H}), 3.15$ $(\mathrm{dd}, J=13.4,6.8 \mathrm{~Hz}, 1 \mathrm{H}), 3.55(\mathrm{dd}, J=13.4,7.3 \mathrm{~Hz}, 1 \mathrm{H}), 5.27(\mathrm{t}, J=7.0 \mathrm{~Hz}, 1 \mathrm{H}), 6.65$ (dt, $J=7.9,1.1 \mathrm{~Hz}, 2 \mathrm{H}), 6.76(\mathrm{tt}, J=7.4,1.1 \mathrm{~Hz}, 1 \mathrm{H}), 7.16-7.25(\mathrm{~m}, 4 \mathrm{H}), 7.27-7.36(\mathrm{~m}, 3 \mathrm{H})$, 7.63-7.68 (m, 2H), $7.73(\mathrm{dd}, J=7.8,1.7 \mathrm{~Hz}, 1 \mathrm{H})$.

${ }^{13} \mathrm{C}-\mathrm{NMR}\left(76 \mathrm{MHz}, \mathrm{CDCl}_{3}\right) \delta=5.0,-5.0,16.3,21.6,25.9,47.2,52.3,91.9,101.8,113.5$, $118.2,127.2,128.5,128.7,129.4,129.5,130.5,132.5,134.1,135.4,136.0,143.9,146.8$.

IR (ATR): $\tilde{v}\left(\mathrm{~cm}^{-1}\right)=3413(\mathrm{w}), 2933$ (m), $2891(\mathrm{w}), 2857$ (m), 1602 (m), $\left.1506 \mathrm{~m}\right), 1468$ (m), $1356(\mathrm{~m}), 1253(\mathrm{~m}), 1164(\mathrm{~s}), 1080(\mathrm{~m})$.

ESI-HRMS: $\mathrm{C}_{29} \mathrm{H}_{35} \mathrm{BrN}_{2} \mathrm{O}_{2} \mathrm{SSi} m / z$ calcd. $\left[\mathrm{M}+\mathrm{H}^{+}\right]$: 583.1445 found 583.1447. 


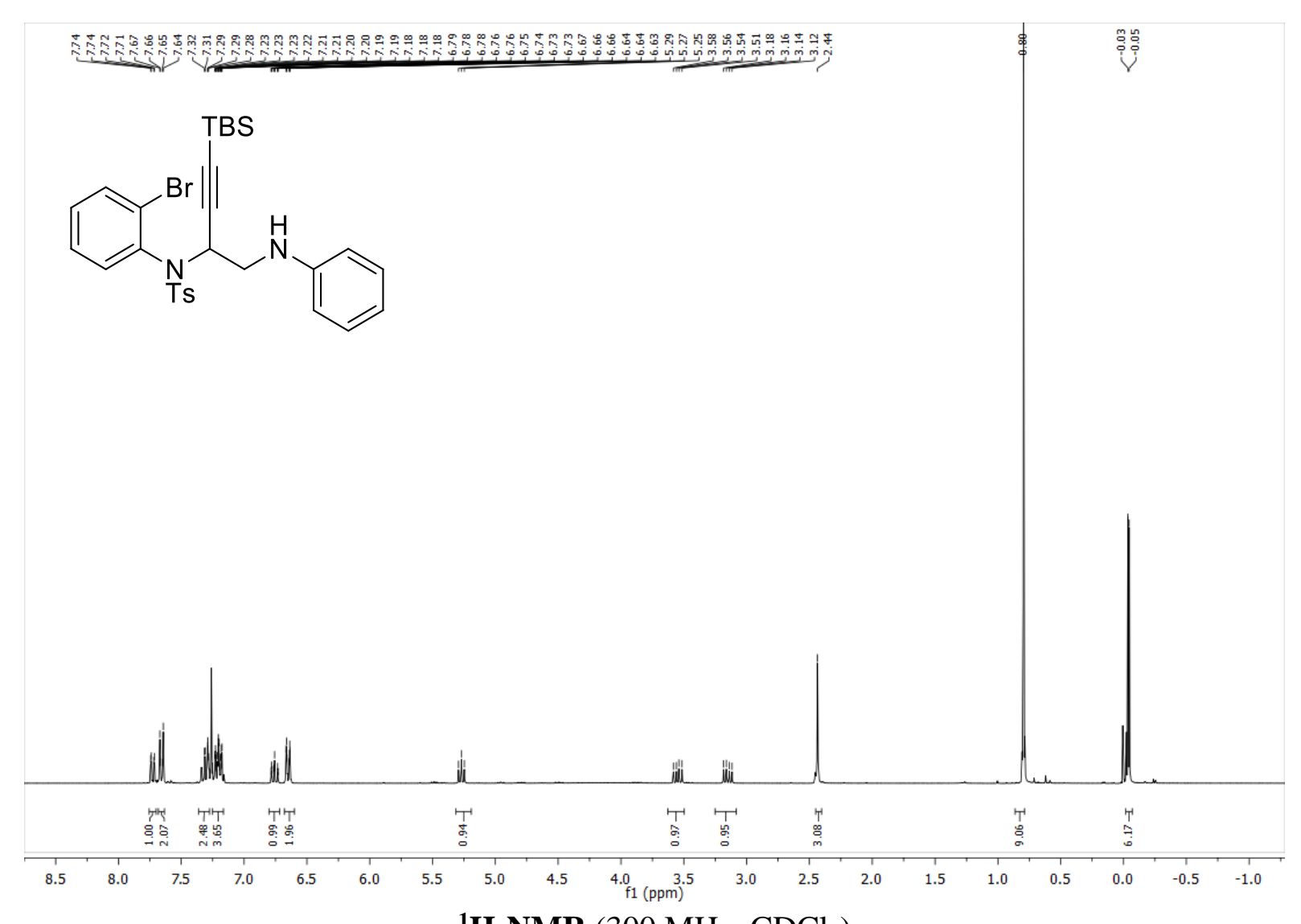

${ }^{1} \mathbf{H}$-NMR (300 MHz, $\mathrm{CDCl}_{3}$ )

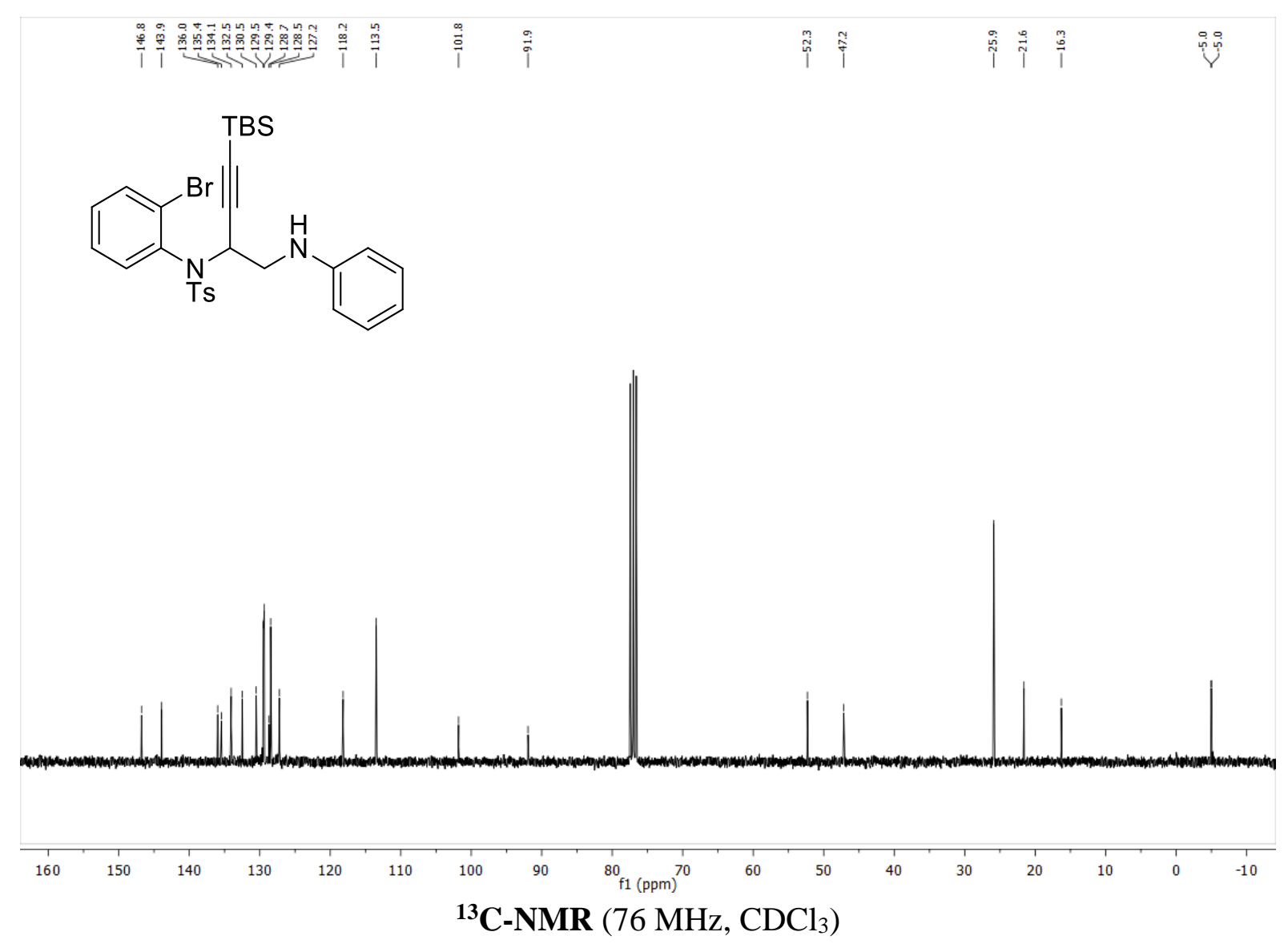




\section{2-(2-Bromophenoxy)- $N$-isopropyl-5,5-dimethylhex-3-yn-1-amine (2i)}<smiles>CC(C)NCC(C#CC(C)(C)C)Oc1ccccc1Br</smiles>

Alcohol $21^{3}$ (297 mg, $\left.1.00 \mathrm{mmol}\right)$ was reacted with DIPEA (250 $\left.\mu \mathrm{L}, 1.44 \mathrm{mmol}\right), \mathrm{Tf}_{2} \mathrm{O}$ $(220 \mu \mathrm{L}, 1.34 \mathrm{mmol})$ and isopropylamine $(250 \mu \mathrm{L}, 2.92 \mathrm{mmol})$ according to GP4 (Synthesis of Amine (B)). The reaction mixture was stirred at ambient temperature overnight. Silica gel column chromatography (EtOAc: $n$-pentane = 1:3) afforded compound $\mathbf{2 i}(221 \mathrm{mg}, 652 \mu \mathrm{mol}$, $65 \%$ ) as yellow oil.

${ }^{1}$ H-NMR (500 MHz, $\mathrm{CDCl}_{3}$ ): $\delta=1.08(\mathrm{~d}, J=6.2 \mathrm{~Hz}, 3 \mathrm{H}), 1.10(\mathrm{~d}, J=6.3 \mathrm{~Hz}, 3 \mathrm{H}), 1.19$ (s, 9H), $2.91(\mathrm{p}, J=6.2 \mathrm{~Hz}, 1 \mathrm{H}), 3.00(\mathrm{dd}, J=12.7,4.2 \mathrm{~Hz}, 1 \mathrm{H}), 3.17(\mathrm{dd}, J=12.7,8.8 \mathrm{~Hz}, 1 \mathrm{H})$, $4.80(\mathrm{dd}, J=8.8,4.2 \mathrm{~Hz}, 1 \mathrm{H}), 6.86(\mathrm{ddd}, J=7.7,6.8,2.0 \mathrm{~Hz}, 1 \mathrm{H}), 7.20-7.27(\mathrm{~m}, 2 \mathrm{H}), 7.52$ $(\mathrm{dd}, J=7.9,1.5 \mathrm{~Hz}, 1 \mathrm{H})$.

${ }^{13}$ C-NMR (126 MHz, $\left.\mathrm{CDCl}_{3}\right) \delta=23.0,23.0,27.4,30.7,47.7,51.8,70.1,75.2,97.0,113.1$, 116.3, 122.6, 128.2, 133.1, 154.5.

IR (ATR): $\tilde{v}\left(\mathrm{~cm}^{-1}\right)=3320(\mathrm{w}), 3067(\mathrm{w}), 2967(\mathrm{~m}), 2929(\mathrm{w}), 2868(\mathrm{w}), 2837(\mathrm{w}), 1584$ (w), $1475(\mathrm{~s}), 1281(\mathrm{~m}), 1240(\mathrm{~m}), 1027(\mathrm{~m})$.

ESI-HRMS: $\mathrm{C}_{17} \mathrm{H}_{24} \mathrm{BrNO} \mathrm{m} / \mathrm{z}$ calcd. $\left[\mathrm{M}+\mathrm{H}^{+}\right]: 338.1114$ found 338.1120 . 


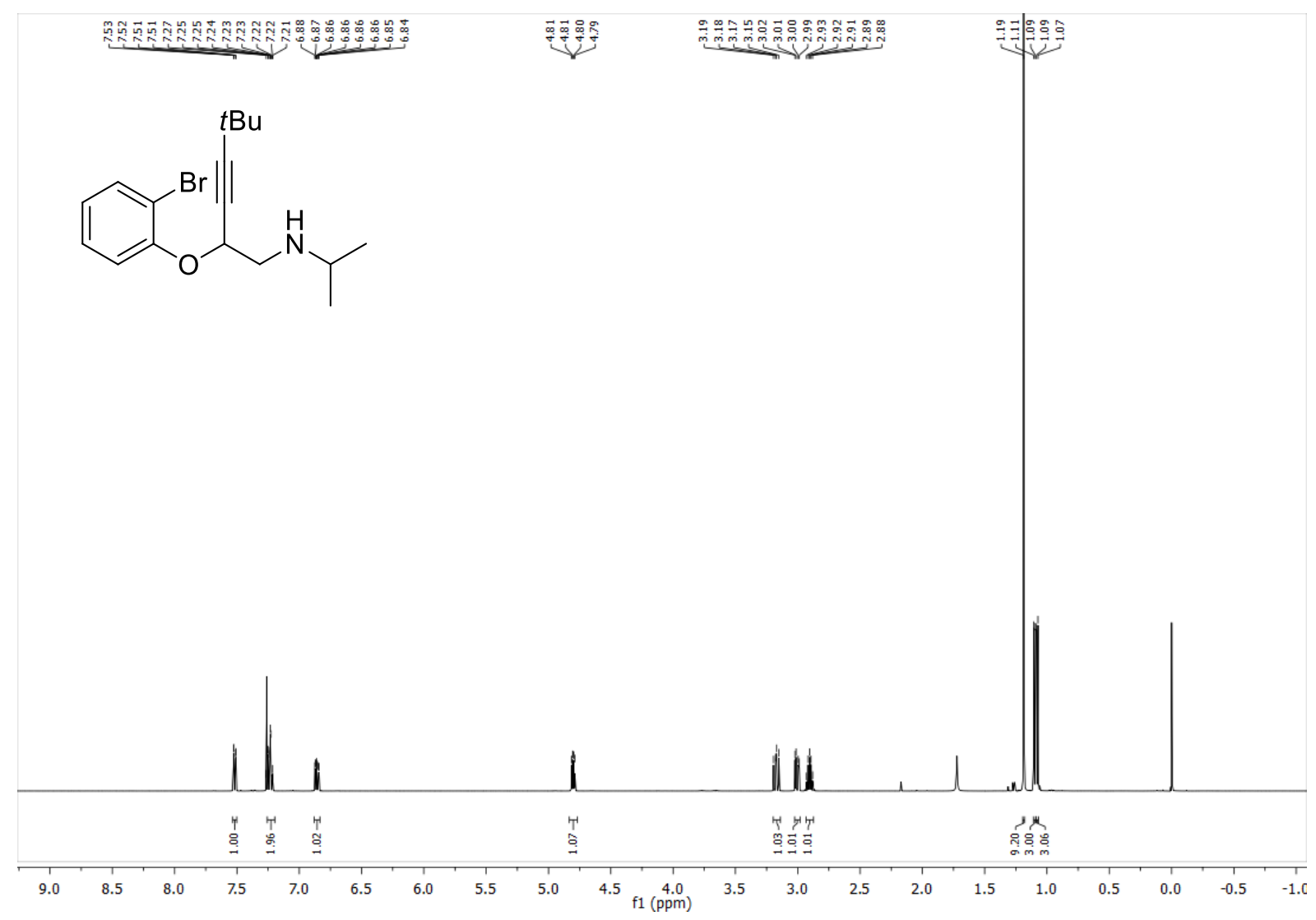

${ }^{1}$ H-NMR (500 MHz, $\mathrm{CDCl}_{3}$ )

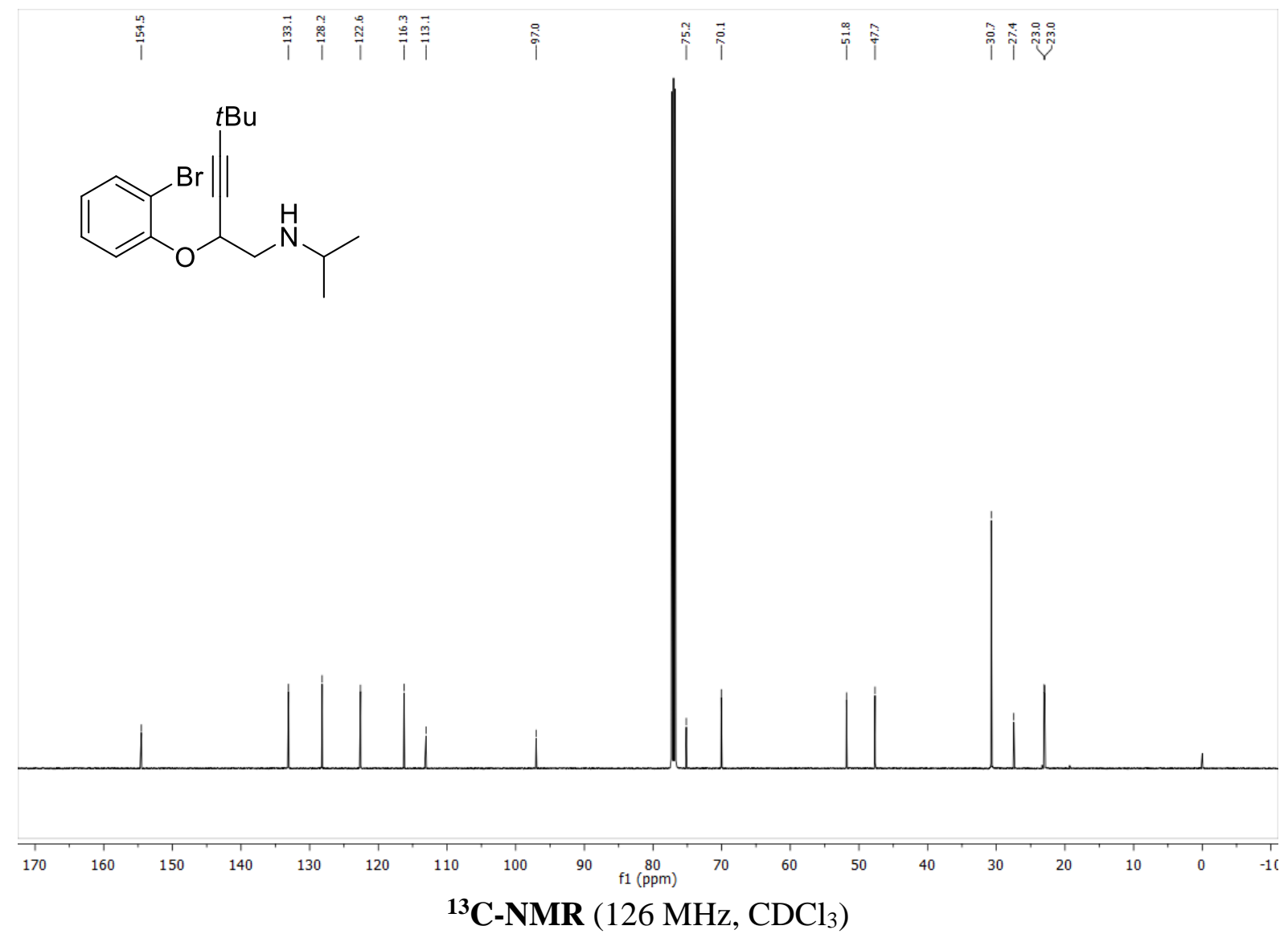




\section{$N$-Benzyl-2-(2-bromophenoxy)-5,5-dimethylhex-3-yn-1-amine (2j)}<smiles>CC(C)(C)C#CC(CNCc1ccccc1)Oc1ccccc1Br</smiles>

Alcohol $21^{3}$ (297 mg, $\left.1.00 \mathrm{mmol}\right)$ was reacted with DIPEA $(250 \mu \mathrm{L}, 1.44 \mathrm{mmol}), \mathrm{Tf}_{2} \mathrm{O}$ $(200 \mu \mathrm{L}, 1.22 \mathrm{mmol})$ and $(300 \mu \mathrm{L}, 2.75 \mathrm{mmol})$ according to GP4 (Synthesis of Amine (B)). The reaction mixture was stirred at ambient temperature overnight. Silica gel column chromatography (EtOAc: $n$-pentane = 1:10) afforded compound 2j (304 mg, 786 mmol, 79\%) as yellow oil.

${ }^{1}$ H-NMR (300 MHz, $\mathrm{CDCl}_{3}$ ): $\delta=1.17$ (s, 9H), 2.07 (s, 1H), $3.00(\mathrm{dd}, J=12.8,4.1 \mathrm{~Hz}, 1 \mathrm{H})$, $3.23(\mathrm{dd}, J=12.8,8.4 \mathrm{~Hz}, 1 \mathrm{H}), 3.90(\mathrm{~s}, 2 \mathrm{H}), 4.85$ (dd, $J=8.4,4.0 \mathrm{~Hz}, 1 \mathrm{H}), 6.86$ (ddd, $J=7.9,6.9,2.0 \mathrm{~Hz}, 1 \mathrm{H}), 7.17-7.25(\mathrm{~m}, 2 \mathrm{H}), 7.25-7.37(\mathrm{~m}, 5 \mathrm{H}), 7.49-7.55(\mathrm{~m}, 1 \mathrm{H})$.

${ }^{13}$ C-NMR $\left(76 \mathrm{MHz}, \mathrm{CDCl}_{3}\right) \delta=27.4,30.7,53.1,53.6,69.9,75.0,97.1,113.1,116.2,122.6$, $126.9,128.0,128.2,128.4,133.1,140.2,154.4$.

IR (ATR): $\tilde{v}\left(\mathrm{~cm}^{-1}\right)=3329(\mathrm{w}), 2969$ (m), $2925(\mathrm{w}), 2902(\mathrm{w}), 2867(\mathrm{w}), 2837(\mathrm{w}), 1584$ (w), $1474(\mathrm{~s}), 1271(\mathrm{~m}), 1239(\mathrm{~s}) 1028(\mathrm{~m})$.

ESI-HRMS: $\mathrm{C}_{21} \mathrm{H}_{24} \mathrm{BrNO} \mathrm{m} / \mathrm{z}$ calcd. $\left[\mathrm{M}+\mathrm{H}^{+}\right]$: 386.1114 found 386.1119 . 


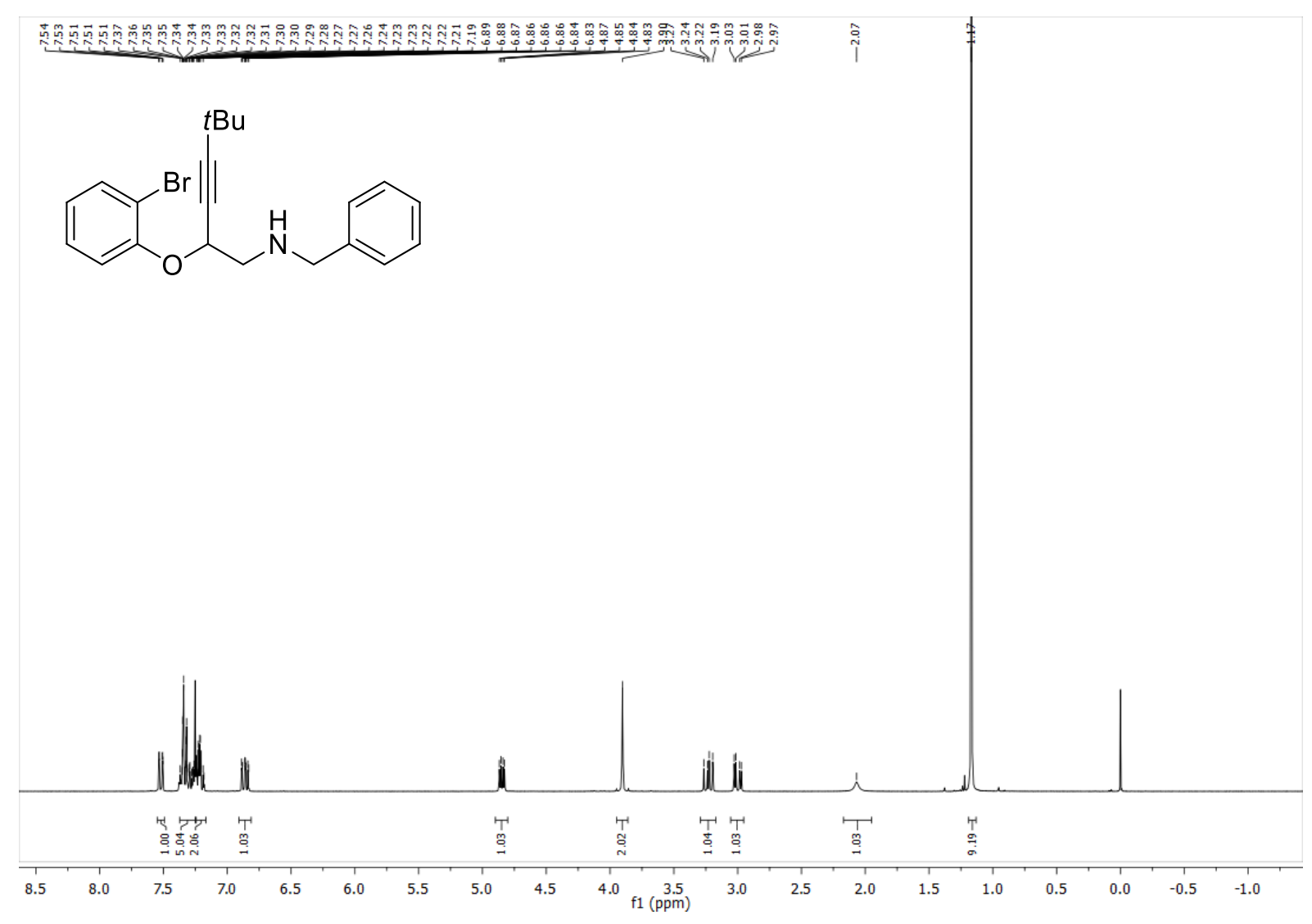

${ }^{1} \mathbf{H}-\mathbf{N M R}\left(300 \mathrm{MHz}, \mathrm{CDCl}_{3}\right)$

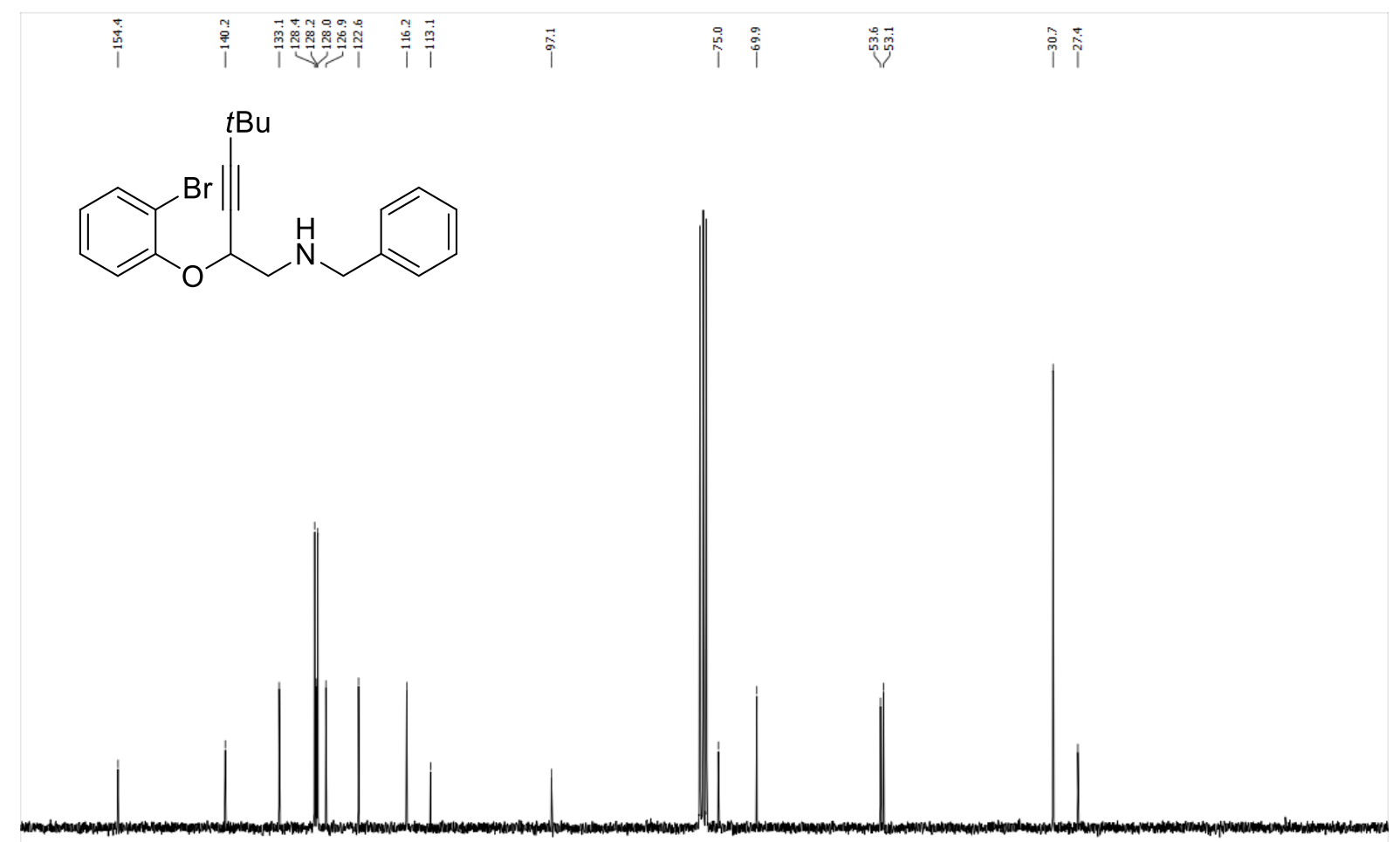

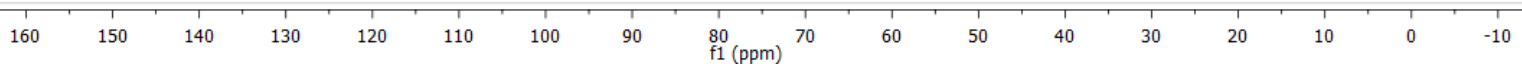

${ }^{13} \mathrm{C}-\mathrm{NMR}\left(76 \mathrm{MHz}, \mathrm{CDCl}_{3}\right)$ 
5) Synthesis of Domino Products of Type 4<smiles>[R][X]1ccc(NCC(C#CC(C)(C)C)Oc2ccccc2Br)cc1</smiles>

$\mathrm{R}=\mathrm{H}$

$\mathrm{R}=3,5-(\mathrm{OMe})_{2} \quad \mathbf{2 b}$

$\mathrm{R}=4-\mathrm{Cl}$

2c

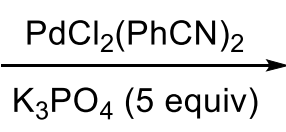<smiles>[R]c1cccc(N2CC3Oc4ccccc4C3=C2C(C)(C)C)c1</smiles>

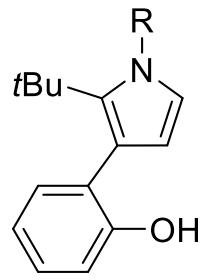

$\mathrm{R}=\mathrm{H}$

$4 \mathbf{a}(58 \%)+5 \mathbf{a}(5 \%)$

$\mathrm{R}=3,5-(\mathrm{OMe})_{2}$

4b $(48 \%)+5 b(23 \%)$

$\mathrm{R}=4-\mathrm{Cl}$

4c $(34 \%)$ 


\section{Domino product (4a)}<smiles>CC(C)(C)C1=C2c3ccccc3OC2CN1c1ccccc1</smiles>

The domino precursor $\mathbf{2 a}(37.2 \mathrm{mg}, 100 \mu \mathrm{mol})$, potassium phosphate $(105 \mathrm{mg}, 495 \mu \mathrm{mol})$, $\left[t \mathrm{Bu}_{3} \mathrm{PH}\right]\left[\mathrm{BF}_{4}\right](2.9 \mathrm{mg}, 10.0 \mu \mathrm{mol})$ and $\mathrm{PdCl}_{2}(\mathrm{PhCN})_{2}(2.0 \mathrm{mg}, 5.21 \mu \mathrm{mol})$ were reacted according to GP3 (Pd-catalyzed Domino Reaction). The mixture was stirred $2 \mathrm{~h}$ at $120^{\circ} \mathrm{C}$. Silica gel column chromatography (EtOAc: $n$-pentane $=1: 50$ ) afforded domino product 4a (16.9 mg, $58.0 \mu \mathrm{mol}, 58 \%)$ as colorless oils and pyrrole 5a $(1.4 \mathrm{mg}, 4.80 \mu \mathrm{mol}, 5 \%)$ as colorless oil.

${ }^{1}$ H-NMR (400 MHz, $\left.\mathrm{CDCl}_{3}\right): \delta=1.18(\mathrm{~s}, 9 \mathrm{H}), 3.68(\mathrm{dd}, J=12.7,7.4 \mathrm{~Hz}, 1 \mathrm{H}), 3.82$ (dd, $J=12.7,2.5 \mathrm{~Hz}, 1 \mathrm{H}), 4.87(\mathrm{dd}, J=7.4,2.5 \mathrm{~Hz}, 1 \mathrm{H}), 6.74-6.82(\mathrm{~m}, 2 \mathrm{H}), 6.93-6.99(\mathrm{~m}, 2 \mathrm{H})$, $7.06(\mathrm{tt}, J=7.5,1.2 \mathrm{~Hz}, 1 \mathrm{H}), 7.20-7.24(\mathrm{~m}, 2 \mathrm{H}), 7.30-7.36(\mathrm{~m}, 2 \mathrm{H})$.

${ }^{13}$ C-NMR (101 MHz, $\left.\mathrm{CDCl}_{3}\right): \delta=27.4,30.7,53.7,64.1,74.1,96.1,117.6,117.6,120.6$, $121.1,123.1,123.4,129.4,131.2,144.3,147.3$.

IR (ATR): $\tilde{v}\left(\mathrm{~cm}^{-1}\right)=2969(\mathrm{~m}), 2926(\mathrm{w}), 2866(\mathrm{w}), 1592(\mathrm{~m}), 1496(\mathrm{~s}), 1352(\mathrm{~m}), 1272(\mathrm{~m})$, 1248 (m).

GC-HRMS (EI): $\mathrm{C}_{20} \mathrm{H}_{21} \mathrm{NO} \mathrm{m} / \mathrm{z}$ calcd. $\left[\mathrm{M}^{+}\right]$: 291.1623 found 291.1613. 


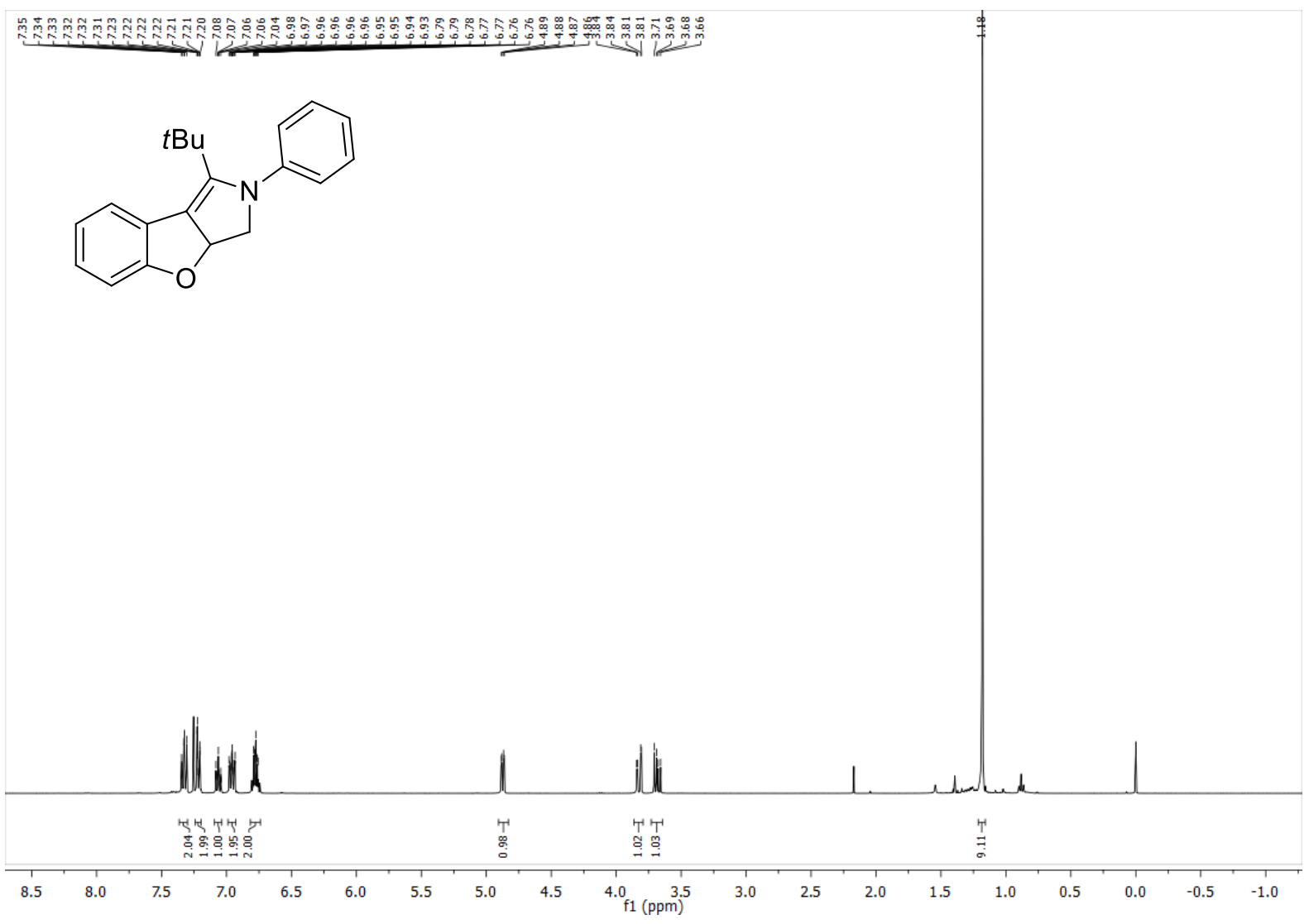

${ }^{1} \mathbf{H}-\mathbf{N M R}\left(400 \mathrm{MHz}, \mathrm{CDCl}_{3}\right)$

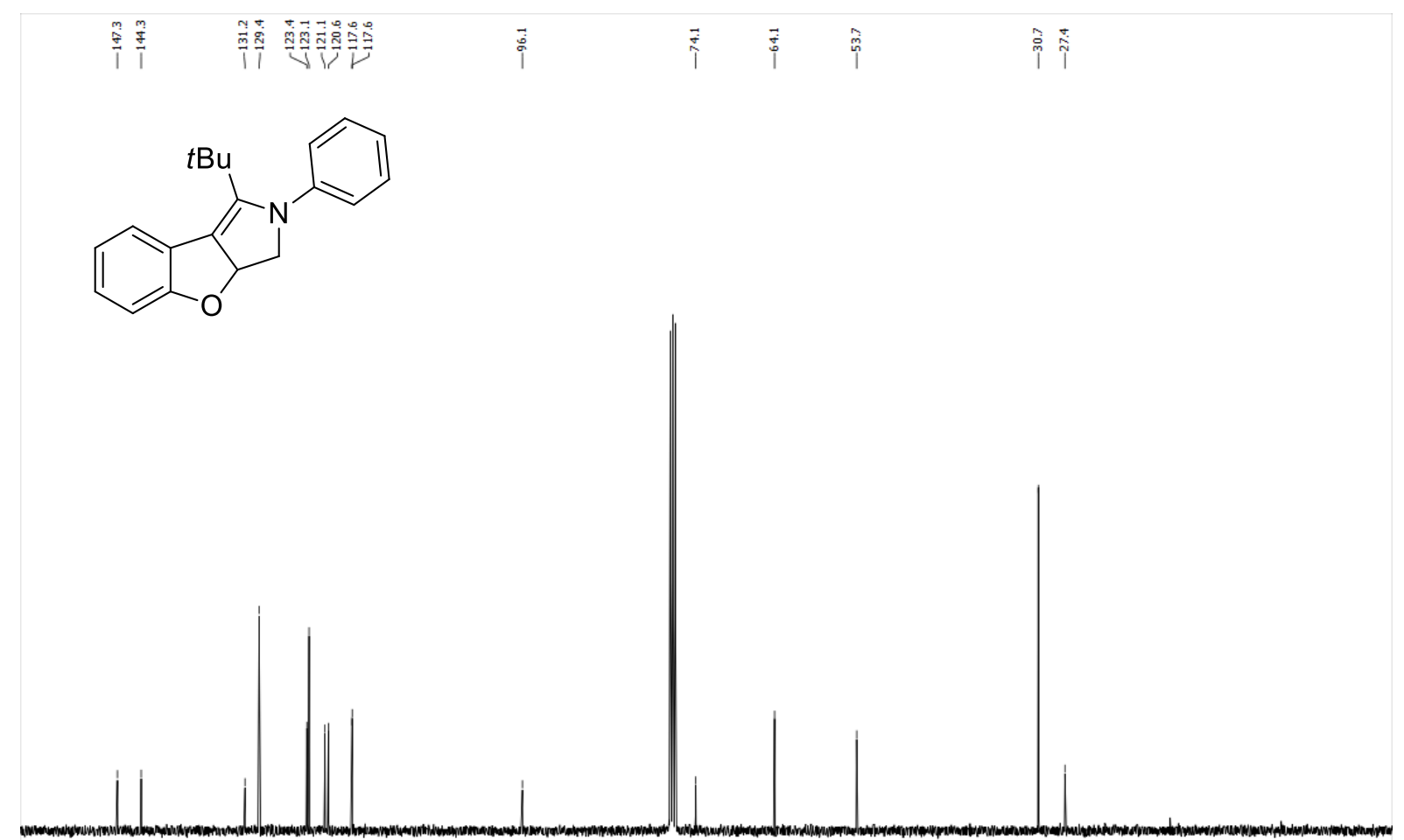

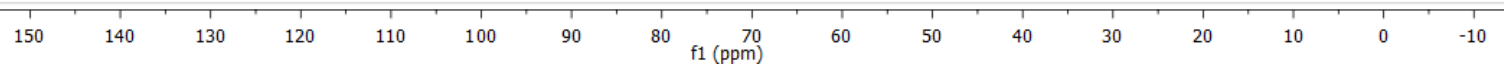

${ }^{13}$ C-NMR $\left(101 \mathrm{MHz}, \mathrm{CDCl}_{3}\right)$ 


\section{Domino product (4b)}<smiles>COc1cc(OC)cc(N2CC3Oc4ccccc4C3=C2C(C)(C)C)c1</smiles>

The domino precursor $\mathbf{2 b}(41.4 \mathrm{mg}, 95.8 \mu \mathrm{mol})$, potassium phosphate $(102 \mathrm{mg}, 480 \mu \mathrm{mol})$, $\left[t \mathrm{Bu}_{3} \mathrm{PH}\right]\left[\mathrm{BF}_{4}\right](2.9 \mathrm{mg}, 10.0 \mu \mathrm{mol})$ and $\mathrm{PdCl}_{2}(\mathrm{PhCN})_{2}(2.0 \mathrm{mg}, 5.21 \mu \mathrm{mol})$ were reacted according to GP3 (Pd-catalyzed Domino Reaction). The mixture was stirred $2 \mathrm{~h}$ at $120{ }^{\circ} \mathrm{C}$. Silica gel column chromatography (EtOAc: $n$-pentane $=1: 20$ ) afforded domino product $\mathbf{4 b}$ (16.3 mg, $46.4 \mu \mathrm{mol}, 48 \%)$ as colorless oil and pyrrole $\mathbf{5 b}(7.9 \mathrm{mg}, 22.5 \mu \mathrm{mol}, 23 \%)$ as yellow oil.

${ }^{1}$ H-NMR (300 MHz, $\left.\mathrm{CDCl}_{3}\right): \delta=1.19$ (s, 9H), 3.64 (dd, $\left.J=12.7,7.4 \mathrm{~Hz}, 1 \mathrm{H}\right), 3.76(\mathrm{~s}, 6 \mathrm{H})$, $3.83(\mathrm{dd}, J=12.7,2.5 \mathrm{~Hz}, 1 \mathrm{H}), 4.85(\mathrm{dd}, J=7.5,2.5 \mathrm{~Hz}, 1 \mathrm{H}), 6.19$ (t, $J=2.2 \mathrm{~Hz}, 1 \mathrm{H}), 6.37$ $(\mathrm{d}, J=2.3 \mathrm{~Hz}, 2 \mathrm{H}), 6.74-6.84(\mathrm{~m}, 2 \mathrm{H}), 6.92-6.98(\mathrm{~m}, 1 \mathrm{H}), 7.01-7.07(\mathrm{~m}, 1 \mathrm{H})$.

${ }^{13}$ C-NMR (76 MHz, $\left.\mathrm{CDCl}_{3}\right): \delta=27.4,30.7,53.6,55.4,64.3,74.0,95.4,96.1,101.1,117.6$, $118.5,121.0,121.0,130.7,144.5,149.3,161.4$.

IR (ATR): $\tilde{v}\left(\mathrm{~cm}^{-1}\right)=2966(\mathrm{~m}), 2866(\mathrm{w}), 2842(\mathrm{w}), 1589(\mathrm{~s}), 1494(\mathrm{~m}), 1455(\mathrm{~m}), 1202(\mathrm{~s})$, $1153(\mathrm{~s}), 1059(\mathrm{~m})$.

GC-HRMS (EI): $\mathrm{C}_{22} \mathrm{H}_{25} \mathrm{NO}_{3} \mathrm{~m} / z$ calcd. $\left[\mathrm{M}^{+}\right]$: 351.1834 found 351.1813 . 


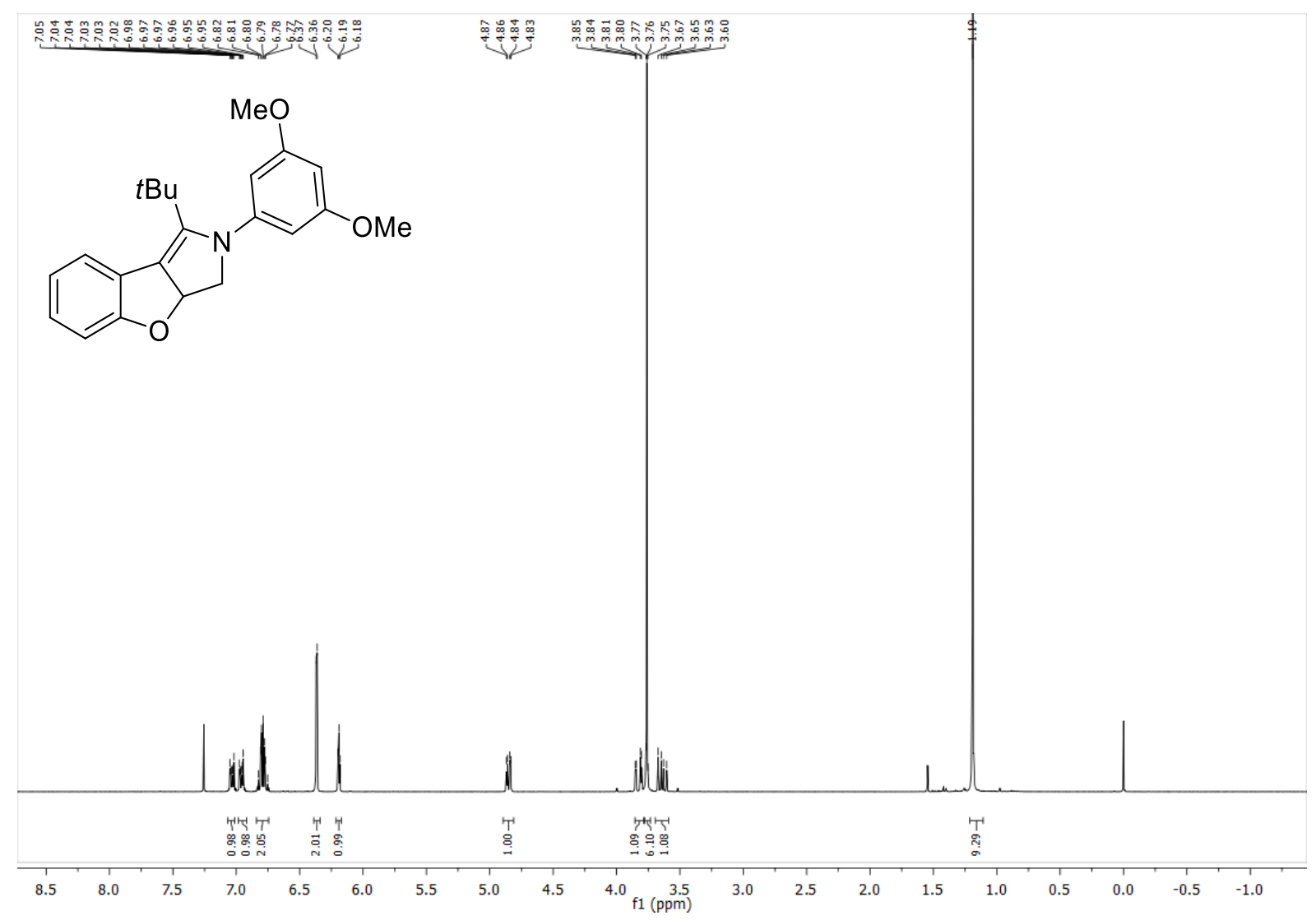

${ }^{1} \mathbf{H}-\mathbf{N M R}\left(300 \mathrm{MHz}, \mathrm{CDCl}_{3}\right)$
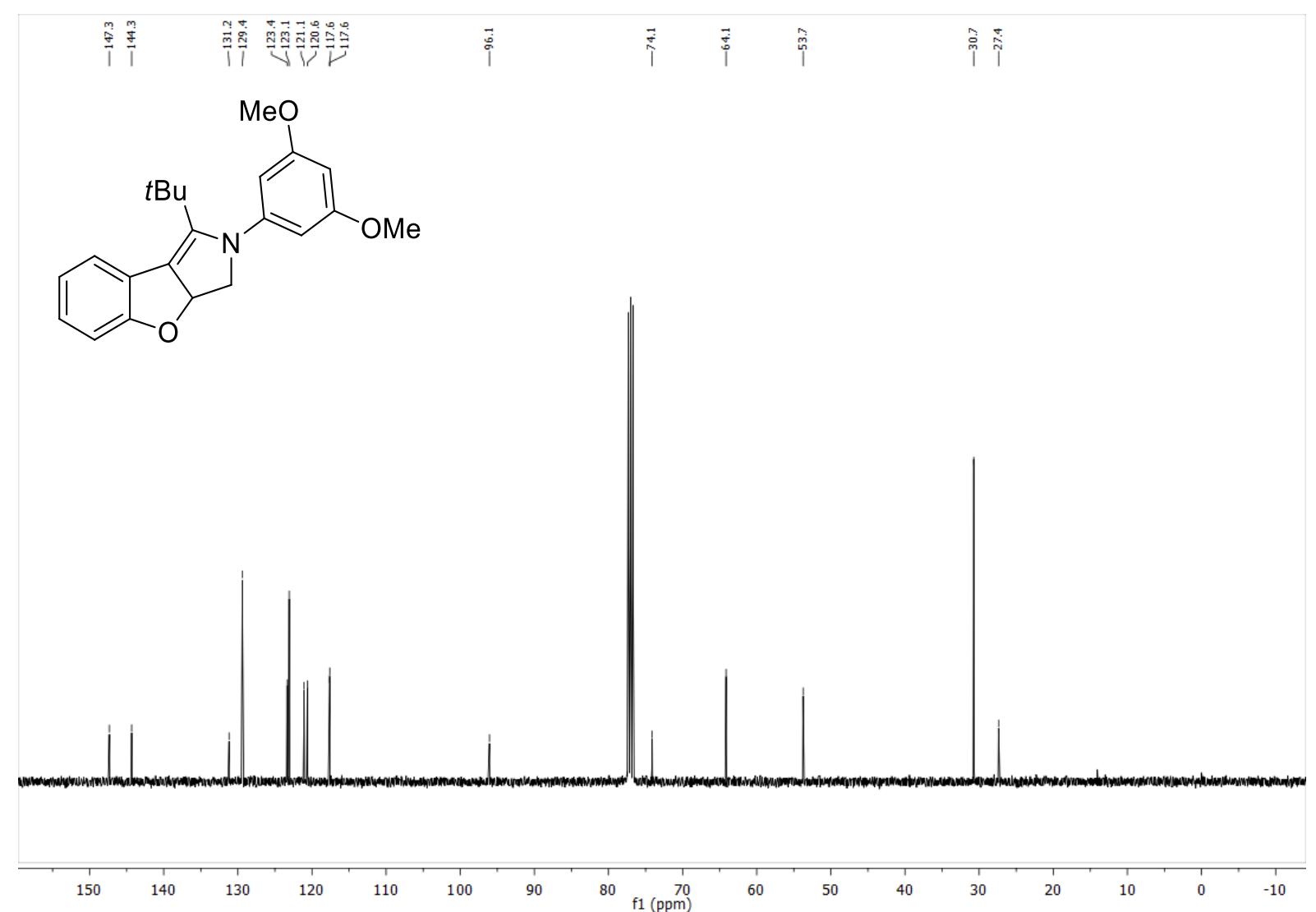

${ }^{13} \mathbf{C}$-NMR $\left(76 \mathrm{MHz}, \mathrm{CDCl}_{3}\right)$ 


\section{Domino product (4c)}<smiles>CC(C)(C)C1=C2c3ccccc3OC2CN1c1ccc(Cl)cc1</smiles>

The domino precursor $2 \mathrm{c}(48.8 \mathrm{mg}, 120 \mu \mathrm{mol})$, potassium phosphate $(132 \mathrm{mg}, 622 \mu \mathrm{mol})$, $\left[t \mathrm{Bu}_{3} \mathrm{PH}\right]\left[\mathrm{BF}_{4}\right](4.1 \mathrm{mg}, 14.1 \mu \mathrm{mol})$ and $\mathrm{PdCl}_{2}(\mathrm{PhCN})_{2}(2.3 \mathrm{mg}, 6.0 \mu \mathrm{mol})$ were reacted according to GP3 (Pd-catalyzed Domino Reaction). The mixture was stirred $2 \mathrm{~h}$ at $120^{\circ} \mathrm{C}$. Silica gel column chromatography (EtOAc: $n$-pentane $=1: 50$ ) afforded domino product $\mathbf{4 c}$ (13.3 mg, $40.8 \mu \mathrm{mol}, 34 \%)$ as colorless oil.

${ }^{1}$ H-NMR (300 MHz, $\left.\mathrm{CDCl}_{3}\right): \delta=1.17(\mathrm{~s}, 9 \mathrm{H}), 3.66(\mathrm{dd}, J=12.6,6.9 \mathrm{~Hz}, 1 \mathrm{H}), 3.77$ (dd, $J=12.7,2.6 \mathrm{~Hz}, 1 \mathrm{H}), 4.87(\mathrm{dd}, J=6.9,2.6 \mathrm{~Hz}, 1 \mathrm{H}), 6.76-6.84(\mathrm{~m}, 2 \mathrm{H}), 6.90-6.98$ (m, 2H), 7.11-7.18 (m, 2H), 7.26-7.30 (m, 2H).

${ }^{13}$ C-NMR (76 MHz, $\left.\mathrm{CDCl}_{3}\right): \delta=27.4,30.7,53.6,64.0,73.9,96.2,117.8,117.8,121.1$, $121.2,124.0,128.2,129.4,130.6,144.4,146.0$.

IR (ATR): $\tilde{v}\left(\mathrm{~cm}^{-1}\right)=2968(\mathrm{~m}), 2927(\mathrm{w}), 2900(\mathrm{w}), 2866(\mathrm{w}), 1588(\mathrm{~m}), 1490(\mathrm{~s}), 1460(\mathrm{~m})$, $1246(\mathrm{~s}), 1091(\mathrm{~m}), 1058(\mathrm{~m})$.

GC-HRMS (EI): $\mathrm{C}_{20} \mathrm{H}_{20} \mathrm{ClNO} \mathrm{m} / z$ calcd. $\left[\mathrm{M}^{+}\right]: 325.1233$ found 325.1213 . 

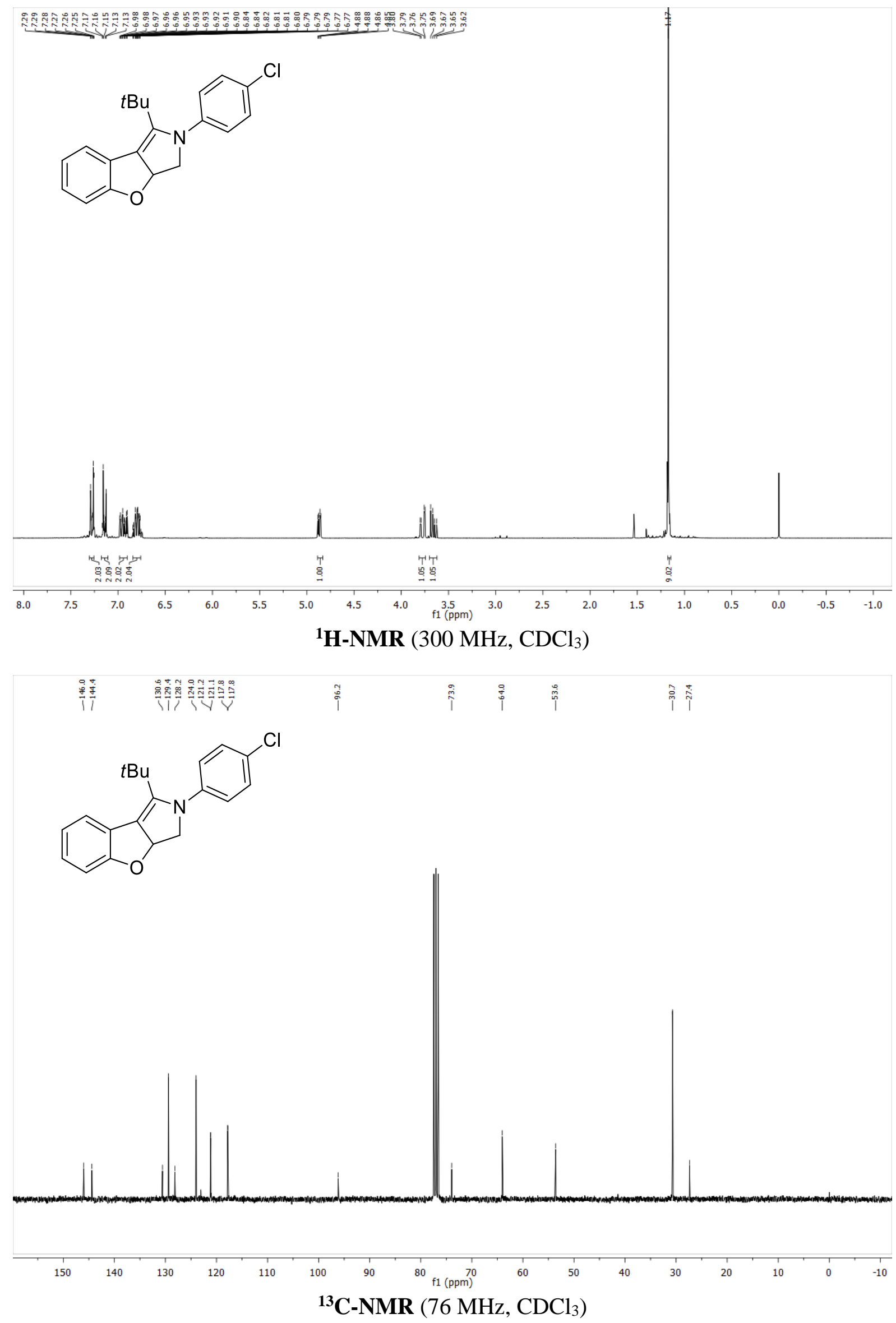

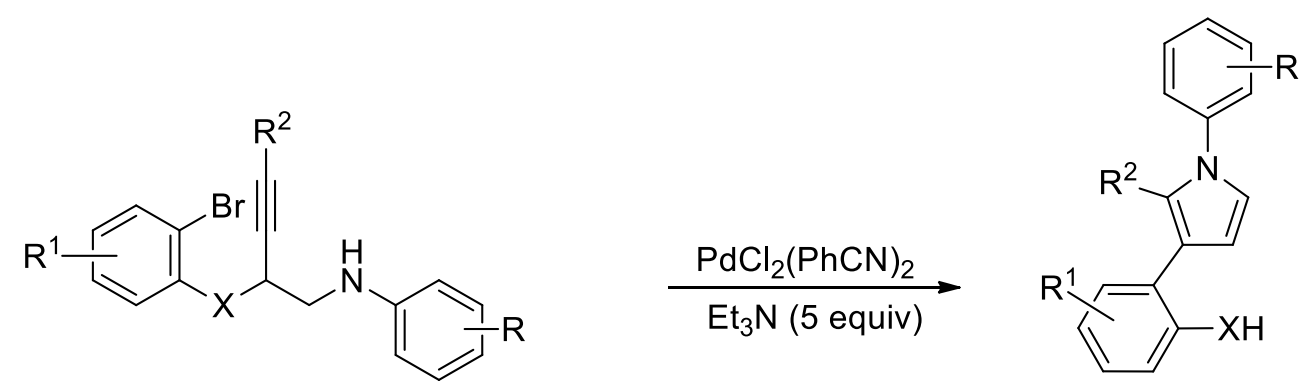

$\mathrm{R}^{1}=\mathrm{H}, \mathrm{R}^{2}=t \mathrm{Bu}, \mathrm{X}=\mathrm{O}, \mathrm{R}=\mathrm{H}$

$2 a$

5a $(78 \%)$

$\mathrm{R}^{1}=\mathrm{H}, \mathrm{R}^{2}=t \mathrm{Bu}, \mathrm{X}=\mathrm{O}, \mathrm{R}=3,5-(\mathrm{OMe})_{2}$

$2 b$

5b $(80 \%)$

$\mathrm{R}^{1}=\mathrm{H}, \mathrm{R}^{2}=t \mathrm{Bu}, \mathrm{X}=\mathrm{O}, \mathrm{R}=4-\mathrm{Cl}$

2c

5 c $(64 \%)$

$\mathrm{R}^{1}=$ naphthyl, $\mathrm{R}^{2}=t \mathrm{Bu}, \mathrm{X}=\mathrm{O}, \mathrm{R}=\mathrm{H} \quad \mathbf{2 d}$

$5 d(66 \%)$

$\mathrm{R}^{1}=\mathrm{H}, \mathrm{R}^{2}=t \mathrm{Bu}, \mathrm{X}=\mathrm{O}, \mathrm{R}=2,4,6-\mathrm{Me}_{3} \quad \mathbf{2 e}$

5 e $(84 \%)$

$\mathrm{R}^{1}=\mathrm{H}, \mathrm{R}^{2}=\mathrm{TMS}, \mathrm{X}=\mathrm{NTs}, \mathrm{R}=2,4,6-\mathrm{Me}_{3} \quad \mathbf{2 f}$

$5 \mathbf{f}(50 \%)$

$\mathrm{R}^{1}=\mathrm{H}, \mathrm{R}^{2}=\mathrm{TMS}, \mathrm{X}=\mathrm{NTs}, \mathrm{R}=\mathrm{H}$

$5 g(58 \%)$

$\mathrm{R}^{1}=\mathrm{H}, \mathrm{R}^{2}=$ TBS, $\mathrm{X}=\mathrm{NTs}, \mathrm{R}=\mathrm{H}$

$2 \mathrm{~h}$

5h (94\%) 


\section{Domino product (5a)}

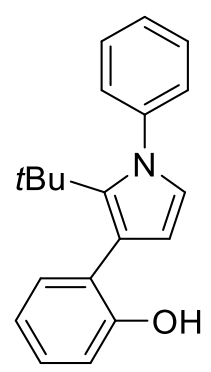

The domino precursor $\mathbf{2 a}(37.2 \mathrm{mg}, 100 \mu \mathrm{mol})$, triethylamine $(70 \mu \mathrm{L}, 505 \mu \mathrm{mol})$, $\left[t \mathrm{Bu}_{3} \mathrm{PH}\right]\left[\mathrm{BF}_{4}\right](2.9 \mathrm{mg}, 10.0 \mu \mathrm{mol})$ and $\mathrm{PdCl}_{2}(\mathrm{PhCN})_{2}(1.9 \mathrm{mg}, 4.95 \mu \mathrm{mol})$ were reacted according to GP3 (Pd-catalyzed Domino Reaction). The mixture was stirred $2 \mathrm{~h}$ at $120^{\circ} \mathrm{C}$. Silica gel column chromatography (EtOAc: $n$-pentane $=1: 50$ ) afforded domino product 5a (22.7 mg, $77.9 \mu \mathrm{mol}, 78 \%$ ) as yellow solid.

${ }^{1}$ H-NMR (400 MHz, $\left.\mathrm{CDCl}_{3}\right): \delta=1.02(\mathrm{~s}, 9 \mathrm{H}), 5.34(\mathrm{~s}, 1 \mathrm{H}), 6.03(\mathrm{~d}, J=2.8 \mathrm{~Hz}, 1 \mathrm{H}), 6.62(\mathrm{~d}$, $J=2.8 \mathrm{~Hz}, 1 \mathrm{H}), 6.86-6.98(\mathrm{~m}, 2 \mathrm{H}), 7.19-7.25(\mathrm{~m}, 2 \mathrm{H}), 7.42-7.44(\mathrm{~m}, 5 \mathrm{H})$.

${ }^{13}$ C-NMR $\left(101 \mathrm{MHz}, \mathrm{CDCl}_{3}\right): \delta=32.6,34.7,111.0,114.4,114.7,119.9,125.2,126.9,128.6$, $128.8,128.9,129.2,131.9,140.3,143.5,154.0$.

IR (ATR): $\tilde{v}\left(\mathrm{~cm}^{-1}\right)=3499(\mathrm{~m}), 2994(\mathrm{w}), 2960$ (m), $2907(\mathrm{w}), 2870(\mathrm{w}), 1597(\mathrm{~m}), 1577$ (m9, $1463(\mathrm{~s}), 1229(\mathrm{~m}), 1184(\mathrm{~m})$.

M.p.: $107 \neg 109^{\circ} \mathrm{C}$.

GC-HRMS (EI): $\mathrm{C}_{20} \mathrm{H}_{21} \mathrm{NO}$ m/z calcd. $\left[\mathrm{M}^{+}\right]$: 291.1623 found 291.1612. 


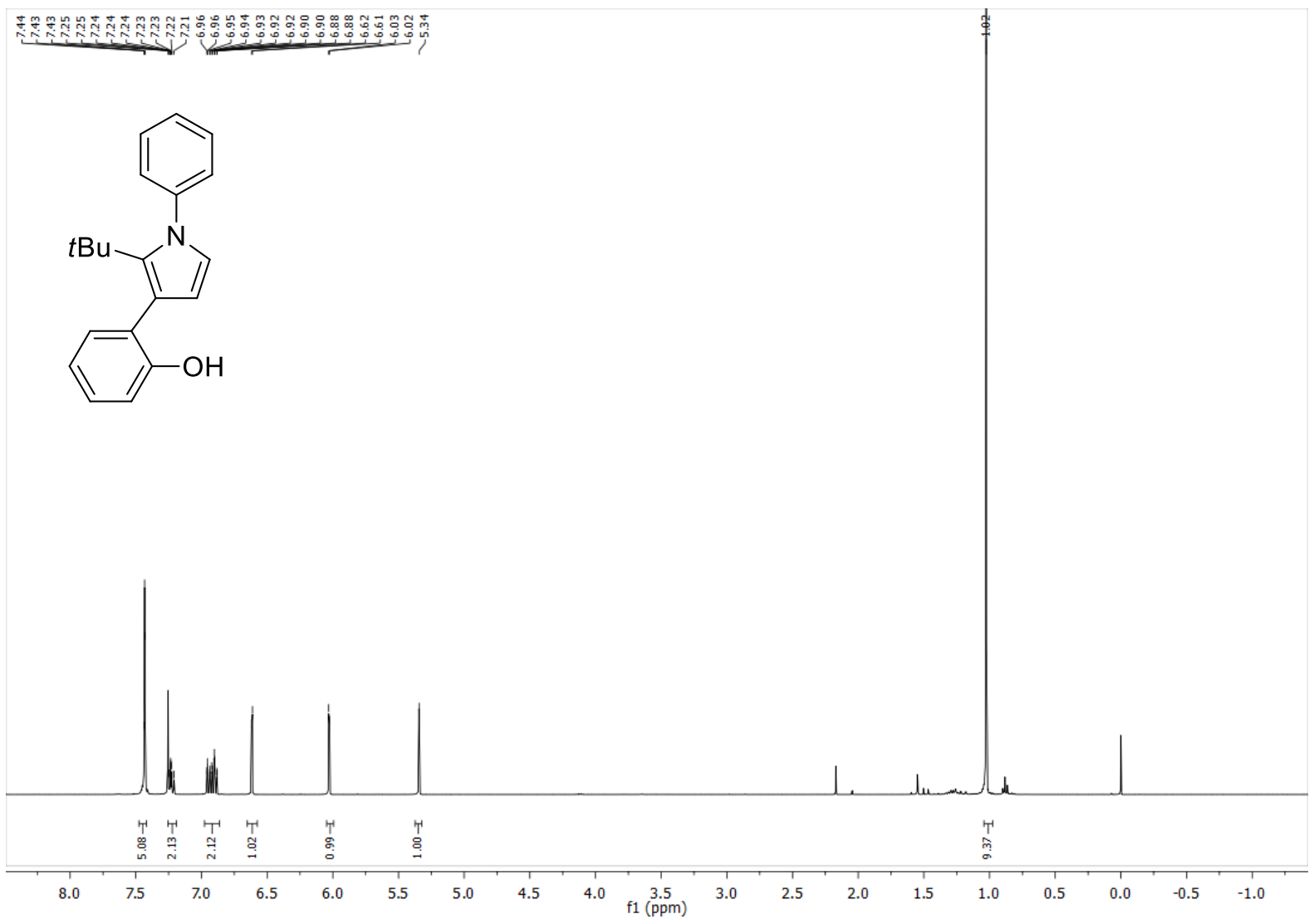

${ }^{1} \mathbf{H}-\mathbf{N M R}\left(400 \mathrm{MHz}, \mathrm{CDCl}_{3}\right)$

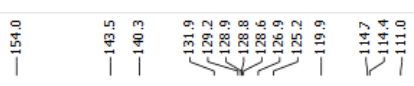<smiles>CC(C)(C)c1c(-c2ccccc2O)ccn1-c1ccccc1</smiles>
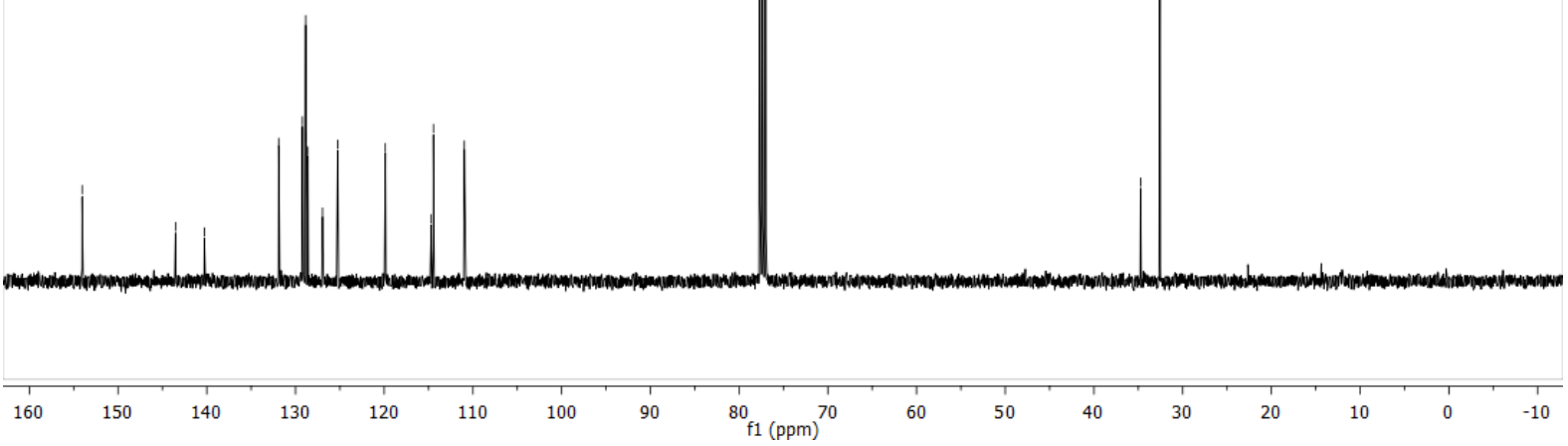

${ }^{13}$ C-NMR (101 MHz, $\left.\mathrm{CDCl}_{3}\right)$ 


\section{Domino product (5b)}

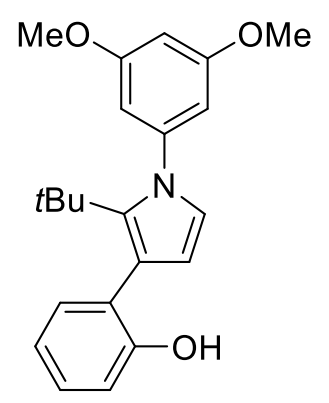

The domino precursor $\mathbf{2 b}(42.5 \mathrm{mg}, 98.3 \mu \mathrm{mol})$, triethylamine $(70 \mu \mathrm{L}, 505 \mu \mathrm{mol})$, $\left[t \mathrm{Bu}_{3} \mathrm{PH}\right]\left[\mathrm{BF}_{4}\right](2.8 \mathrm{mg}, 9.65 \mu \mathrm{mol})$ and $\mathrm{PdCl}_{2}(\mathrm{PhCN})_{2}(2.2 \mathrm{mg}, 5.74 \mu \mathrm{mol})$ were reacted according to GP3 (Pd-catalyzed Domino Reaction). The mixture was stirred $2 \mathrm{~h}$ at $120{ }^{\circ} \mathrm{C}$. Silica gel column chromatography (EtOAc: $n$-pentane $=1: 20$ ) afforded domino product $\mathbf{5 b}$ (27.6 mg, $78.5 \mu \mathrm{mol}, 80 \%$ ) as yellow solid.

${ }^{1}$ H-NMR (300 MHz, $\left.\mathrm{CDCl}_{3}\right): \delta=1.08(\mathrm{~s}, 9 \mathrm{H}), 3.82(\mathrm{~s}, 6 \mathrm{H}), 5.33(\mathrm{~s}, 1 \mathrm{H}), 6.02(\mathrm{~d}, J=2.8 \mathrm{~Hz}$, $1 \mathrm{H}), 6.53(\mathrm{t}, J=2.3 \mathrm{~Hz}, 1 \mathrm{H}), 6.56-6.66(\mathrm{~m}, 3 \mathrm{H}), 6.86-6.96(\mathrm{~m}, 2 \mathrm{H}), 7.19-7.25(\mathrm{~m}, 2 \mathrm{H})$.

${ }^{13}$ C-NMR (76 MHz, $\left.\mathrm{CDCl}_{3}\right): \delta=32.1,34.5,55.6,100.4,107.4,110.6,114.2,114.5,119.6$, 124.6, 126.6, 128.5, 131.6, 139.6, 144.8, 153.7, 160.4 .

IR (ATR): $\tilde{v}\left(\mathrm{~cm}^{-1}\right)=3477(\mathrm{~m}), 2996$ (w), 2955 (m), 2904 (w), 2867 (w), 1595 (s), 1476 (m), $1460(\mathrm{~m}), 1238(\mathrm{~m}), 1200(\mathrm{~m}), 1155$ (s), 1057 (s).

M.p.: $151 \neg 154{ }^{\circ} \mathrm{C}$.

GC-HRMS (EI): $\mathrm{C}_{22} \mathrm{H}_{25} \mathrm{NO}_{3} \mathrm{~m} / \mathrm{z}$ calcd. $\left[\mathrm{M}^{+}\right]$: 351.1834 found 351.1813 . 


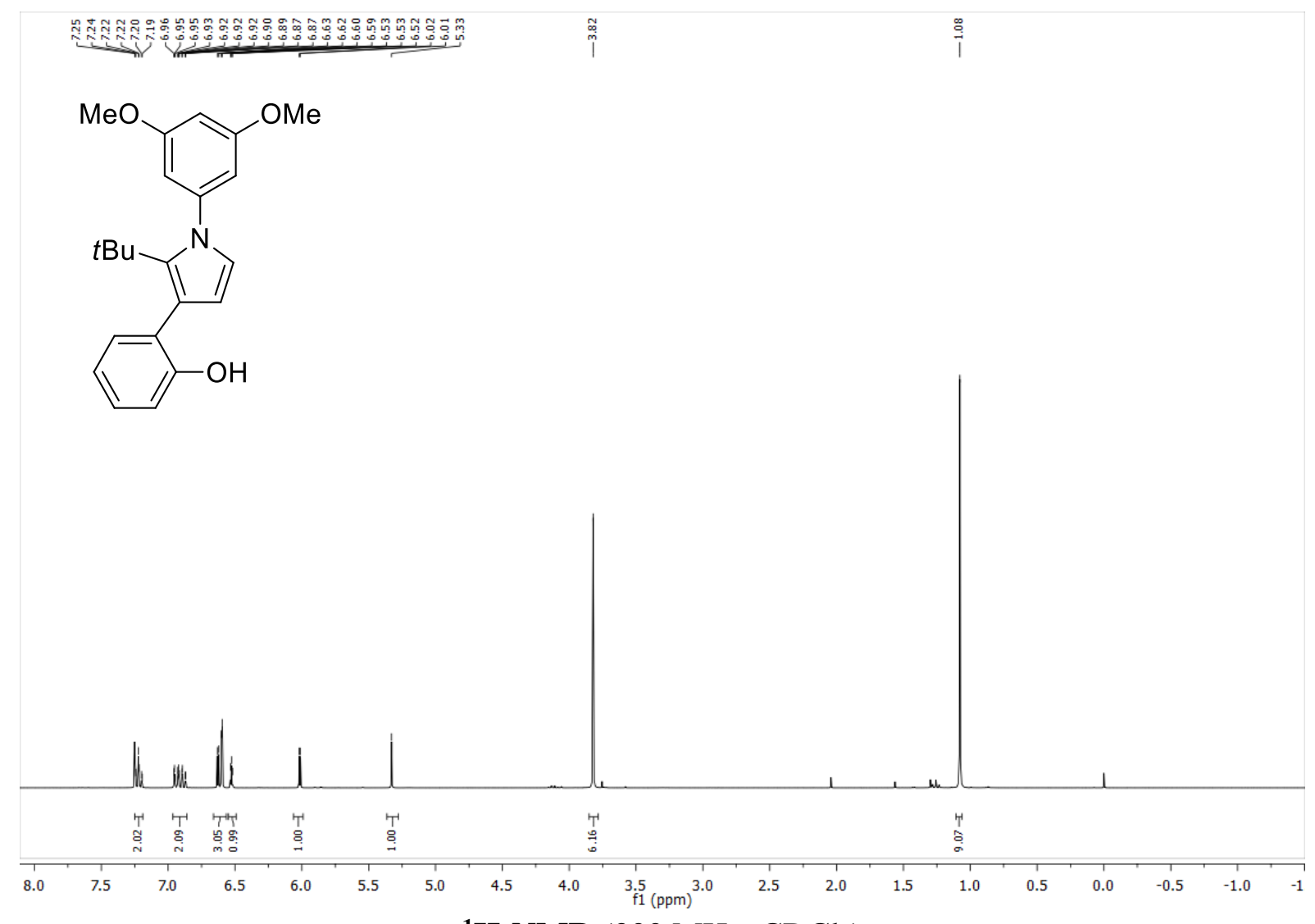

${ }^{1} \mathbf{H}-\mathbf{N M R}\left(300 \mathrm{MHz}, \mathrm{CDCl}_{3}\right.$ )
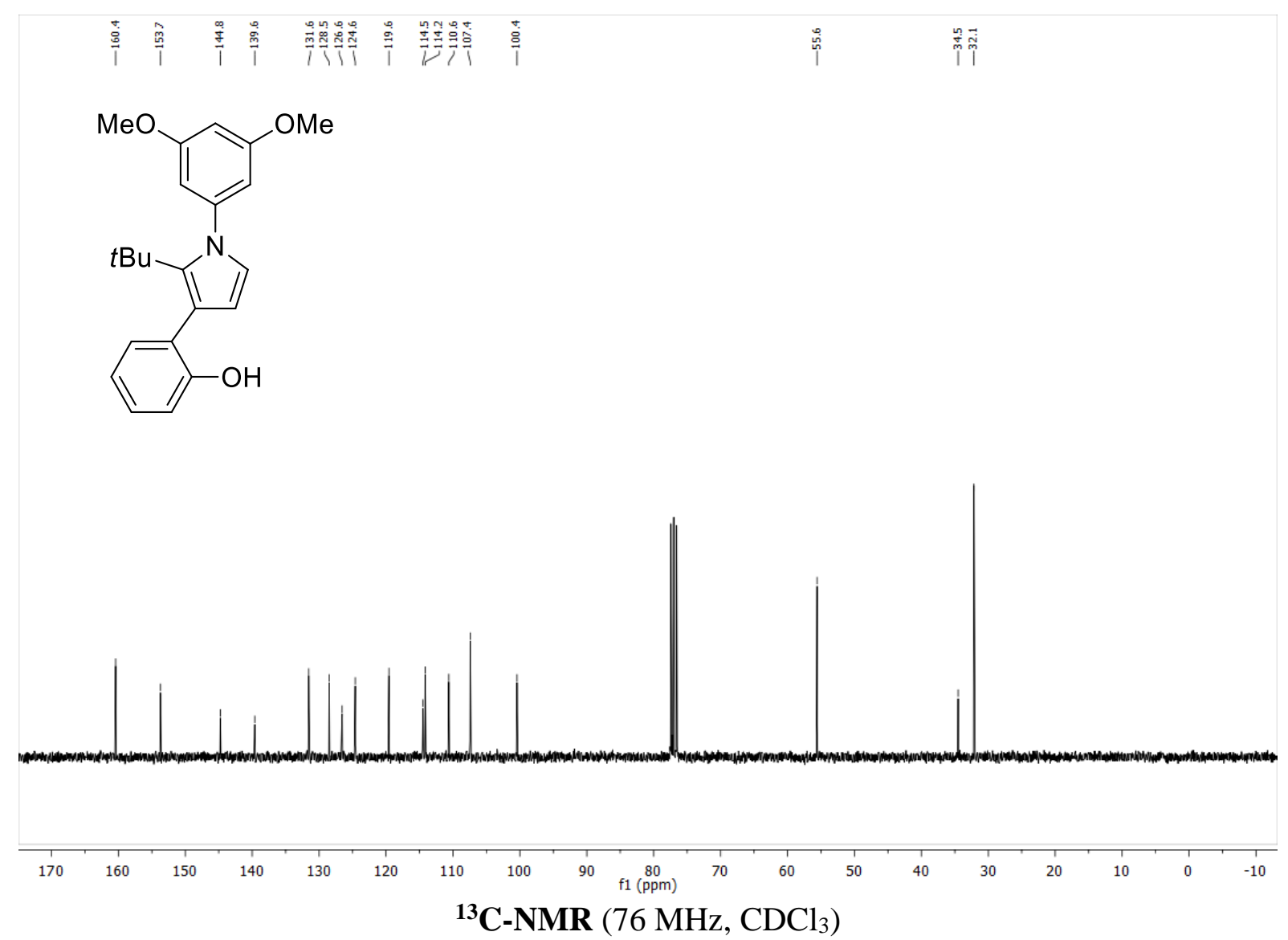


\section{Domino product $(5 \mathrm{c})$}

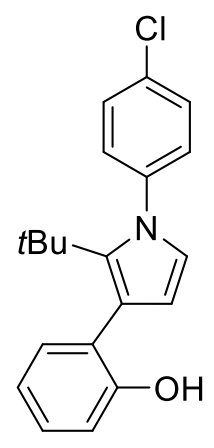

The domino precursor $2 \mathrm{c}(48.8 \mathrm{mg}, 120 \mu \mathrm{mol})$, triethylamine $(90 \mu \mathrm{L}, 649 \mu \mathrm{mol})$, $\left[t \mathrm{Bu}_{3} \mathrm{PH}\right]\left[\mathrm{BF}_{4}\right](3.8 \mathrm{mg}, 13.1 \mu \mathrm{mol})$ and $\mathrm{PdCl}_{2}(\mathrm{PhCN})_{2}(2.5 \mathrm{mg}, 6.52 \mu \mathrm{mol})$ were reacted according to GP3 (Pd-catalyzed Domino Reaction). The mixture was stirred $2 \mathrm{~h}$ at $120^{\circ} \mathrm{C}$. Silica gel column chromatography (EtOAc: $n$-pentane $=1: 50)$ afforded domino product $\mathbf{5 c}$ (25.0 mg, $76.7 \mu \mathrm{mol}, 64 \%$ ) as yellow solid.

${ }^{1}$ H-NMR (300 MHz, $\left.\mathrm{CDCl}_{3}\right): \delta=1.03(\mathrm{~s}, 9 \mathrm{H}), 5.28(\mathrm{~s}, 1 \mathrm{H}), 6.03(\mathrm{~d}, J=2.8 \mathrm{~Hz}, 1 \mathrm{H}), 6.57(\mathrm{~d}$, $J=2.8 \mathrm{~Hz}, 1 \mathrm{H}), 6.87-6.96(\mathrm{~m}, 2 \mathrm{H}), 7.22(\mathrm{dt}, J=7.3,1.5 \mathrm{~Hz}, 2 \mathrm{H}), 7.34-7.43(\mathrm{~m}, 4 \mathrm{H})$.

${ }^{13}$ C-NMR $\left(76 \mathrm{MHz}, \mathrm{CDCl}_{3}\right) \delta=32.3,34.4,111.1,114.2,114.8,119.6,124.8,126.3,128.6$, $128.8,130.1,131.5,134.2,140.1,141.7,153.7$.

IR (ATR): $\tilde{v}\left(\mathrm{~cm}^{-1}\right)=3500(\mathrm{~m}), 2991(\mathrm{w}), 2962(\mathrm{~m}), 2910(\mathrm{w}), 2871(\mathrm{w}), 1494(\mathrm{~s}), 1539(\mathrm{~m})$, $1229(\mathrm{~m}), 1182(\mathrm{~m})$.

M.p.: $112 \neg 116^{\circ} \mathrm{C}$.

GC-HRMS (EI): $\mathrm{C}_{20} \mathrm{H}_{20} \mathrm{ClNO} \mathrm{m} / z$ calcd. $\left[\mathrm{M}^{+}\right]$: 325.1233 found 325.1209 . 


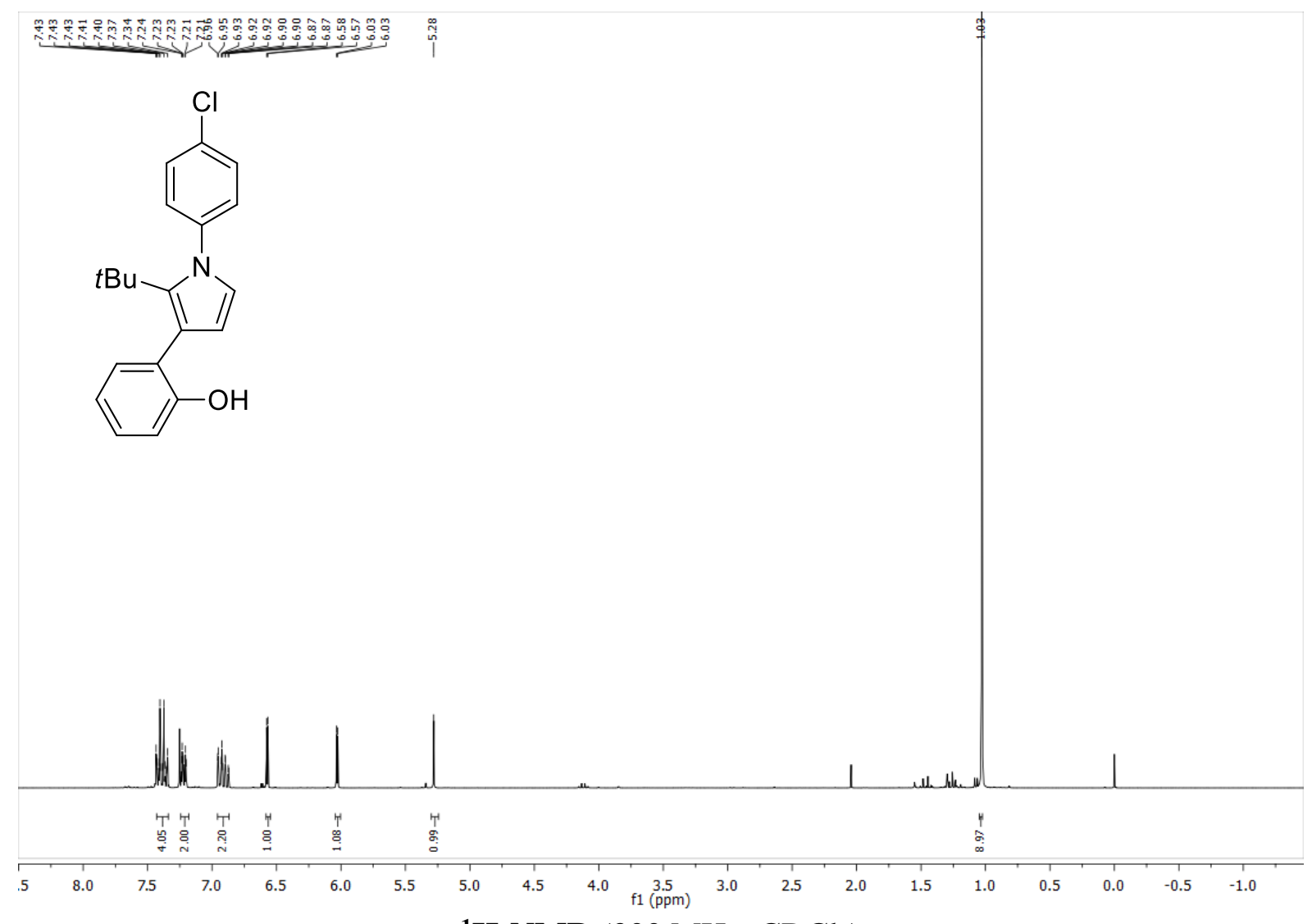

${ }^{1} \mathbf{H}$-NMR (300 MHz, $\mathrm{CDCl}_{3}$ )

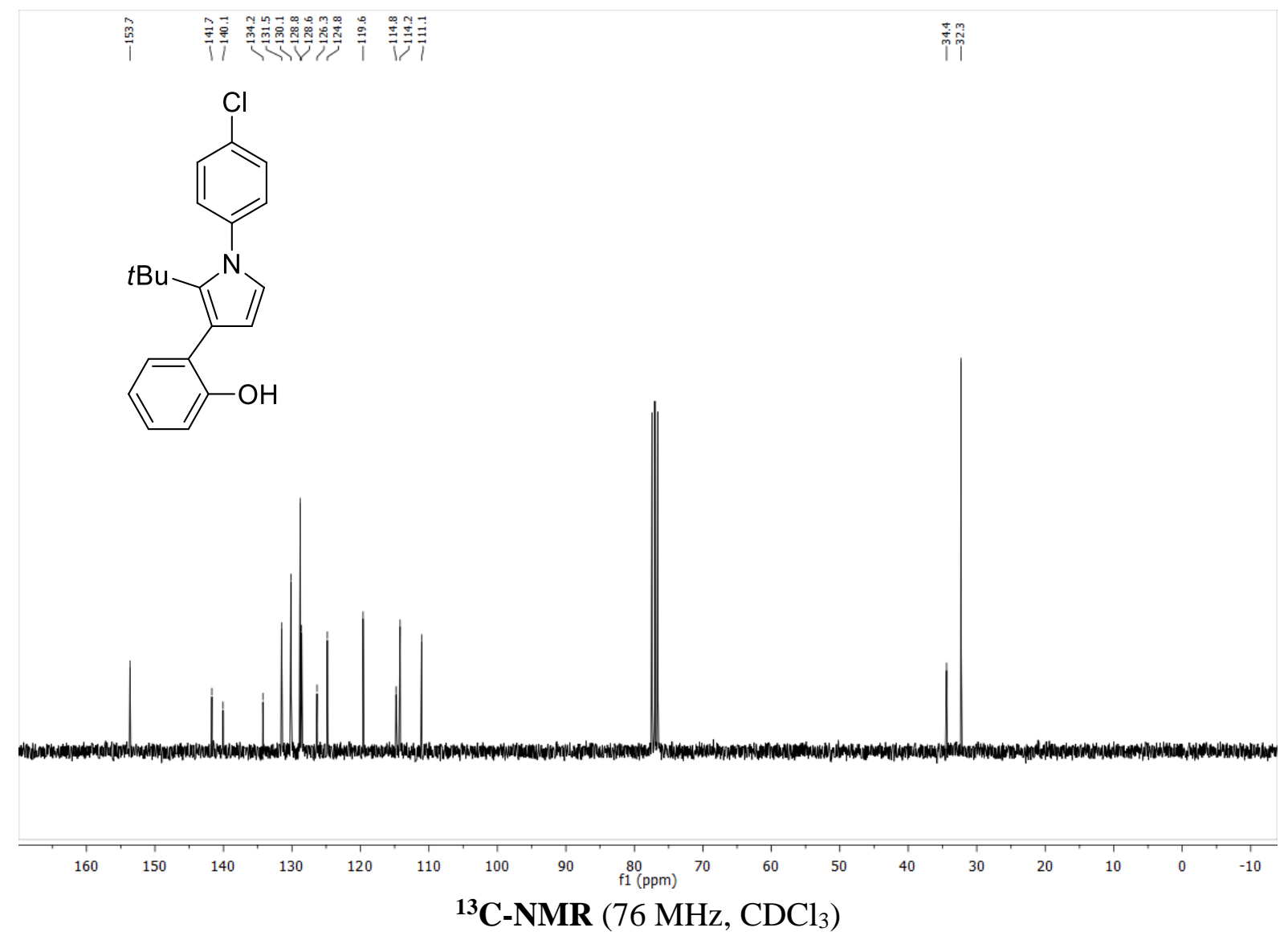




\section{Domino product (5d)}

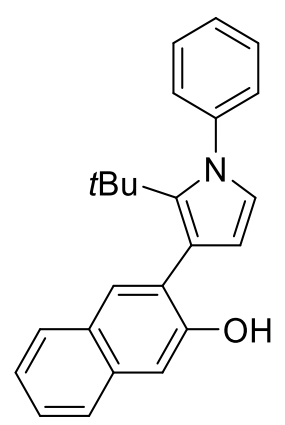

The domino precursor $2 d(30.0 \mathrm{mg}, 71.0 \mu \mathrm{mol})$, triethylamine $(50 \mu \mathrm{L}, 361 \mu \mathrm{mol})$, $\left[t \mathrm{Bu}_{3} \mathrm{PH}\right]\left[\mathrm{BF}_{4}\right](2.1 \mathrm{mg}, 7.24 \mu \mathrm{mol})$ and $\mathrm{PdCl}_{2}(\mathrm{PhCN})_{2}(1.5 \mathrm{mg}, 3.91 \mu \mathrm{mol})$ were reacted according to GP3 (Pd-catalyzed Domino Reaction). The mixture was stirred $2 \mathrm{~h}$ at $120{ }^{\circ} \mathrm{C}$. Silica gel column chromatography (EtOAc: $n$-pentane $=1: 20$ ) afforded domino product 5d (16.0 mg, $46.9 \mu \mathrm{mol}, 66 \%)$ as yellow oil.

${ }^{1} \mathbf{H}-\mathrm{NMR}\left(500 \mathrm{MHz}, \mathrm{CDCl}_{3}\right): \delta=1.04(\mathrm{~s}, 9 \mathrm{H}), 5.53(\mathrm{~s}, 1 \mathrm{H}), 6.08(\mathrm{~d}, J=2.8 \mathrm{~Hz}, 1 \mathrm{H}), 6.65(\mathrm{~d}$, $J=3.0 \mathrm{~Hz}, 1 \mathrm{H}), 7.29-7.34(\mathrm{~m}, 2 \mathrm{H}), 7.37-7.42(\mathrm{~m}, 1 \mathrm{H}), 7.45$ (s, 5H), 7.71-7.78 (m, 3H).

${ }^{13}$ C-NMR (126 MHz, $\left.\mathrm{CDCl}_{3}\right): \delta=32.3,34.5,108.4,111.0,114.0,123.3,125.1,125.9,126.4$, $127.6,128.4,128.4,128.6,128.9,129.2,130.3,134.4,140.3,143.1,152.3$.

IR (ATR): $\tilde{v}\left(\mathrm{~cm}^{-1}\right)=3487(\mathrm{~m}), 2994(\mathrm{w}), 2961(\mathrm{~m}), 2907$ (w), $2870(\mathrm{w}), 1500(\mathrm{~s}), 1453(\mathrm{~m})$, $1260(\mathrm{~m}), 1244(\mathrm{~m}), 1165$ (m).

ESI-HRMS: $\mathrm{C}_{24} \mathrm{H}_{23} \mathrm{NO} \mathrm{m} / \mathrm{z}$ calcd. $\left[\mathrm{M}+\mathrm{H}^{+}\right]$: 342.1852 found 342.1854 . 


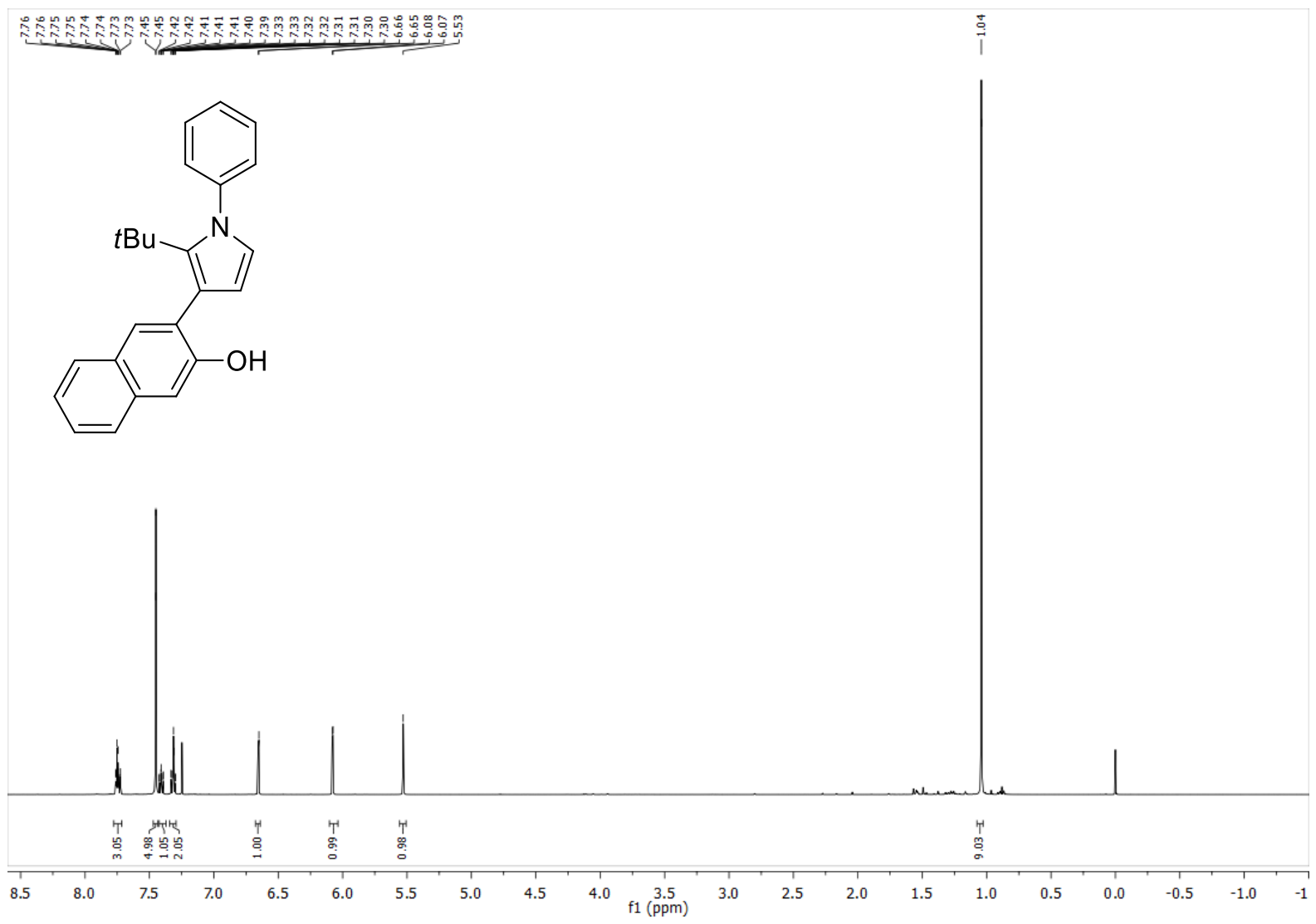

${ }^{1} \mathbf{H}$-NMR (500 MHz, $\mathrm{CDCl}_{3}$ )

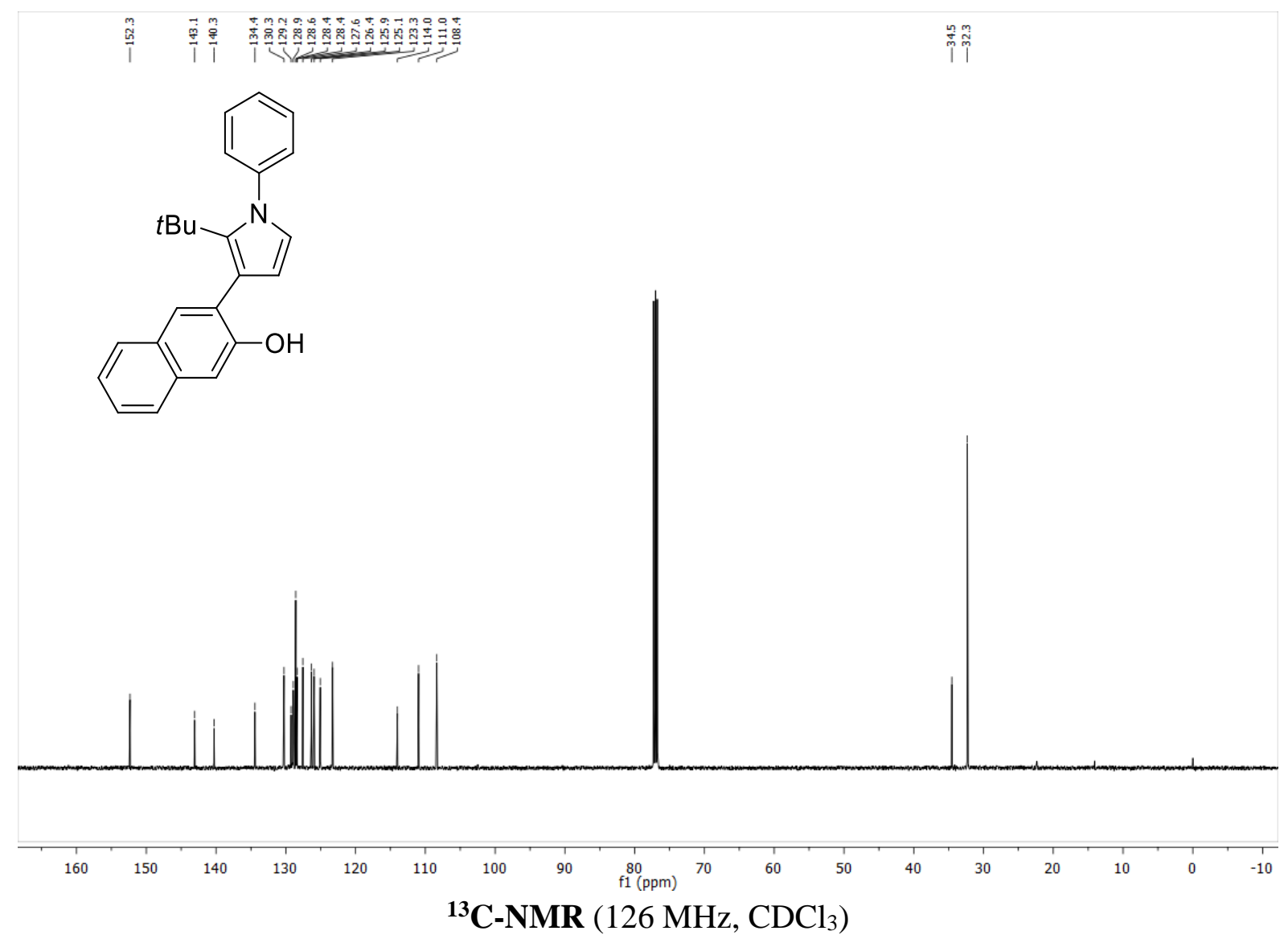




\section{Domino product (5e)}

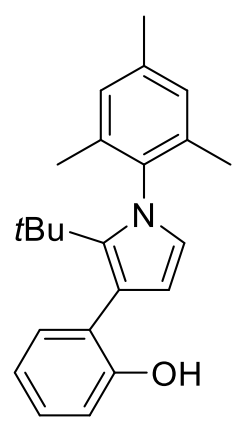

The domino precursor $2 \mathrm{e}(78.0 \mathrm{mg}, 188 \mu \mathrm{mol})$, triethylamine $(130 \mu \mathrm{L}, 938 \mu \mathrm{mol})$, $\left[t \mathrm{Bu}_{3} \mathrm{PH}\right]\left[\mathrm{BF}_{4}\right](5.5 \mathrm{mg}, 19.0 \mu \mathrm{mol})$ and $\mathrm{PdCl}_{2}(\mathrm{PhCN})_{2}(3.6 \mathrm{mg}, 9.39 \mu \mathrm{mol})$ were reacted according to GP3 (Pd-catalyzed Domino Reaction). The mixture was stirred $2 \mathrm{~h}$ at $120{ }^{\circ} \mathrm{C}$. Silica gel column chromatography (EtOAc: $n$-pentane $=1: 50$ ) afforded domino product $\mathbf{5 e}$ (52.8 $\mathrm{mg}, 158 \mu \mathrm{mol}, 84 \%)$ as colorless solid.

${ }^{1}$ H-NMR (400 MHz, $\left.\mathrm{CDCl}_{3}\right): \delta=0.99(\mathrm{~s}, 9 \mathrm{H}), 2.06(\mathrm{~d}, J=23.5 \mathrm{~Hz}, 6 \mathrm{H}), 2.34(\mathrm{~s}, 3 \mathrm{H}), 5.26$ (s, 1H), 6.09 (d, $J=2.8 \mathrm{~Hz}, 1 \mathrm{H}), 6.39$ (d, $J=2.8 \mathrm{~Hz}, 1 \mathrm{H}), 6.92(\mathrm{dtd}, J=13.3,7.8,1.2 \mathrm{~Hz}$, $4 \mathrm{H}), 7.21-7.27(\mathrm{~m}, 2 \mathrm{H})$.

${ }^{13} \mathbf{C}-\mathbf{N M R}\left(101 \mathrm{MHz}, \mathrm{CDCl}_{3}\right): \delta=21.0,31.3,34.4,111.7,114.1,114.2,119.5,122.7,126.6$, $128.5,128.6,128.7,132.0,137.9,138.5,139.1,153.8$.

IR (ATR): $\tilde{v}\left(\mathrm{~cm}^{-1}\right)=3498(\mathrm{~m}), 2991$ (m), $2958(\mathrm{~m}), 2917$ (m), 2868 (m), 1493 (s), 1461 (m), $1227(\mathrm{~m}), 1186(\mathrm{~m})$.

M.p.: $92 \neg 95^{\circ} \mathrm{C}$.

GC-HRMS (EI): $\mathrm{C}_{23} \mathrm{H}_{27} \mathrm{NO} \mathrm{m} / z$ calcd. $\left[\mathrm{M}^{+}\right]: 333.2093$ found 333.2079. 


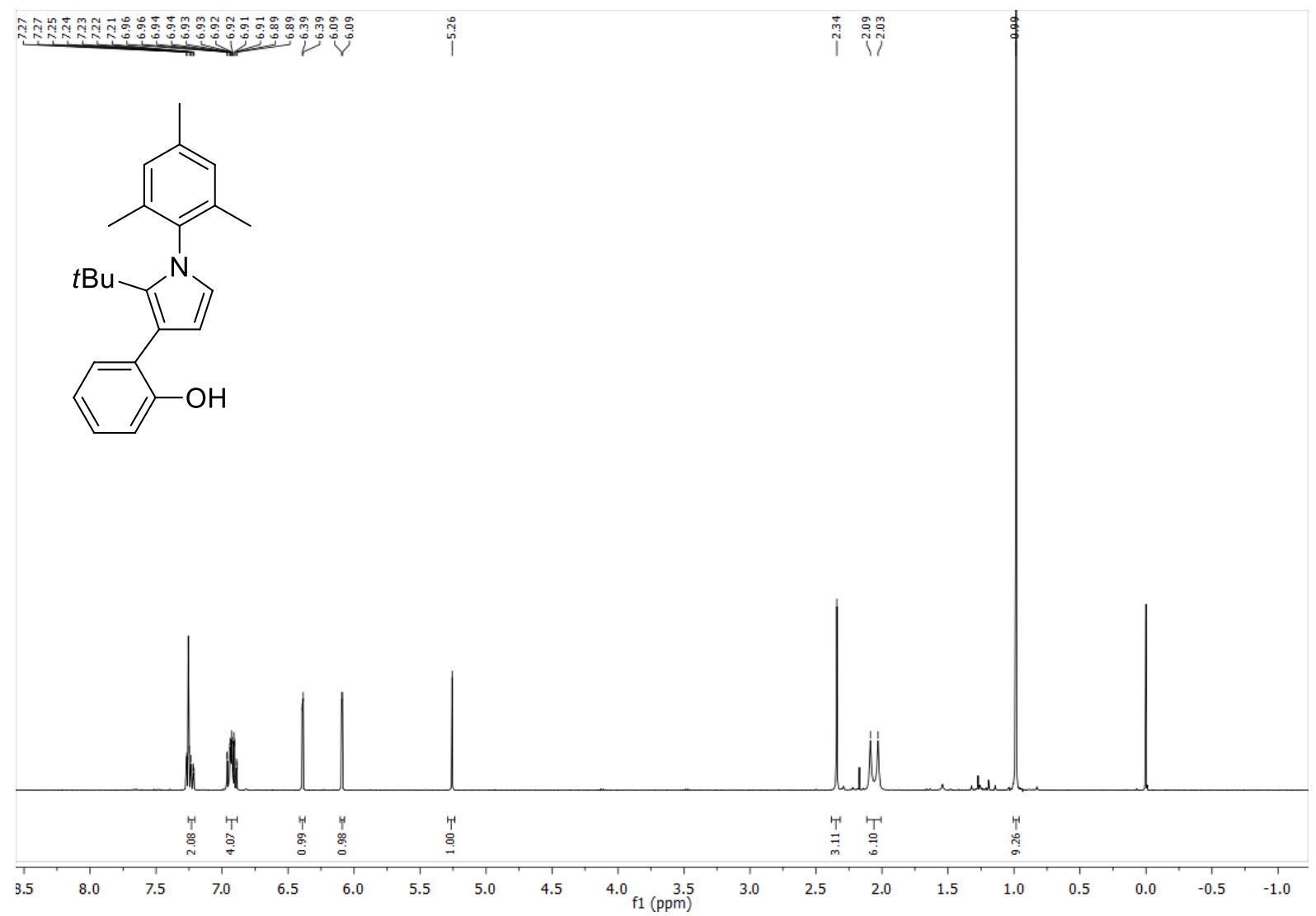

${ }^{1} \mathbf{H}-\mathbf{N M R}\left(400 \mathrm{MHz}, \mathrm{CDCl}_{3}\right)$

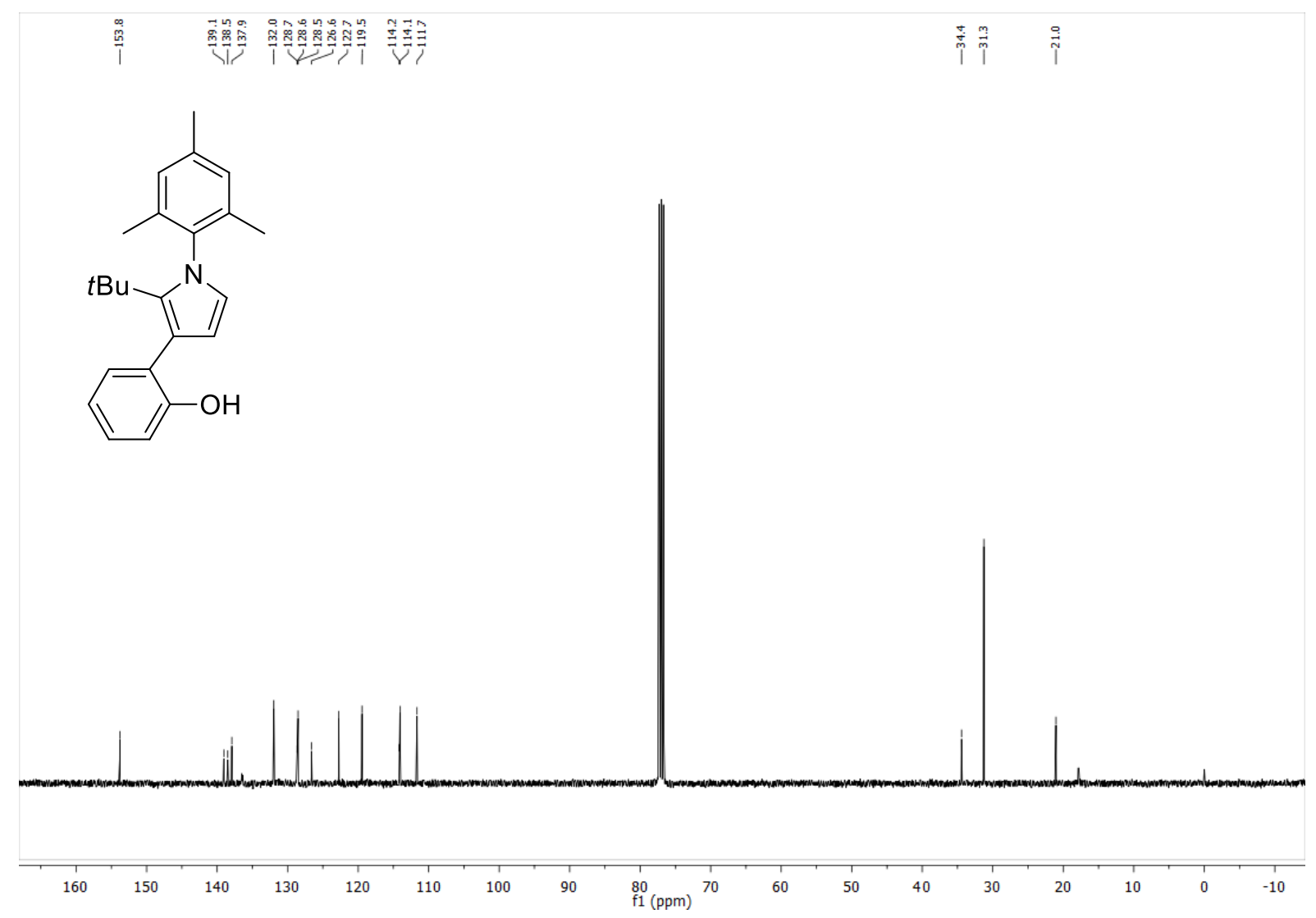

${ }^{13}$ C-NMR (101 MHz, $\left.\mathrm{CDCl}_{3}\right)$ 


\section{Domino product (5f)}

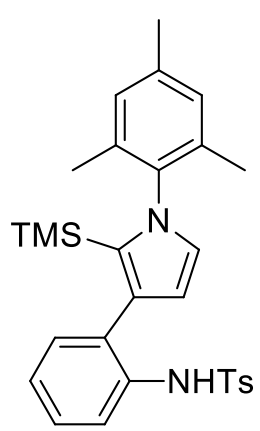

$5 \mathrm{fa}$

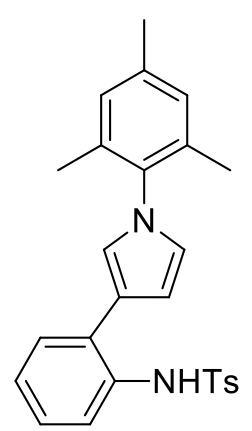

$5 \mathrm{fb}$

The domino precursor 2 f $(46.0 \mathrm{mg}, 78.8 \mu \mathrm{mol})$, triethylamine $(55 \mu \mathrm{L}, 397 \mu \mathrm{mol})$, $\left[t \mathrm{Bu}_{3} \mathrm{PH}\right]\left[\mathrm{BF}_{4}\right](2.3 \mathrm{mg}, 7.93 \mu \mathrm{mol})$ and $\mathrm{PdCl}_{2}(\mathrm{PhCN})_{2}(1.5 \mathrm{mg}, 3.91 \mu \mathrm{mol})$ were reacted according to GP3 (Pd-catalyzed Domino Reaction). The mixture was stirred $2 \mathrm{~h}$ at $120^{\circ} \mathrm{C}$. Silica gel column chromatography (EtOAc: $n$-pentane $=1: 20)$ afforded a mixture of domino product $\mathbf{5 f a}(19.7 \mathrm{mg}, 39.2 \mu \mathrm{mol}, 50 \%)$ as colorless oil and domino product $\mathbf{5 f b}(9.6 \mathrm{mg}$, $22.3 \mu \mathrm{mol}, 28 \%$ ) as colorless oil.

Analytical data of $\mathbf{5 f a}$ :

${ }^{1} \mathbf{H}-\mathrm{NMR}\left(300 \mathrm{MHz}, \mathrm{CDCl}_{3}\right): \delta=-0.41(\mathrm{~s}, 9 \mathrm{H}), 2.02(\mathrm{~s}, 6 \mathrm{H}), 2.35(\mathrm{~d}, J=4.2 \mathrm{~Hz}, 6 \mathrm{H}), 5.72$ $(\mathrm{d}, J=2.4 \mathrm{~Hz}, 1 \mathrm{H}), 6.70(\mathrm{~d}, J=2.4 \mathrm{~Hz}, 1 \mathrm{H}), 6.89$ (s, 1H), 6.95 (s, 2H), 7.02 (td, $J=7.4,1.2$ $\mathrm{Hz}, 1 \mathrm{H}), 7.19-7.27$ (m, 3H), 7.58-7.71 (m, 2H).

${ }^{13}$ C-NMR $\left(76 \mathrm{MHz}, \mathrm{CDCl}_{3}\right) \delta=-0.1,17.5,21.2,21.6,111.0,118.1,123.3,127.0,127.5$, $128.3,128.8,129.3,129.6,129.8,130.3,131.9,135.9,136.7,138.2,138.4,143.8$.

IR (ATR): $\tilde{v}\left(\mathrm{~cm}^{-1}\right)=3309(\mathrm{w}), 2956(\mathrm{w}), 2922(\mathrm{w}), 2861(\mathrm{w}), 1492(\mathrm{~m}), 1398(\mathrm{~m}), 1338$ (m), $1247(\mathrm{~m}), 1164(\mathrm{~s}), 1091(\mathrm{~m})$.

ESI-HRMS: $\mathrm{C}_{2} \mathrm{H}_{34} \mathrm{~N}_{2} \mathrm{O}_{2} \mathrm{SSi} \mathrm{m} / z$ calcd. $\left[\mathrm{M}+\mathrm{H}^{+}\right]$: 503.2183 found 503.2186. 


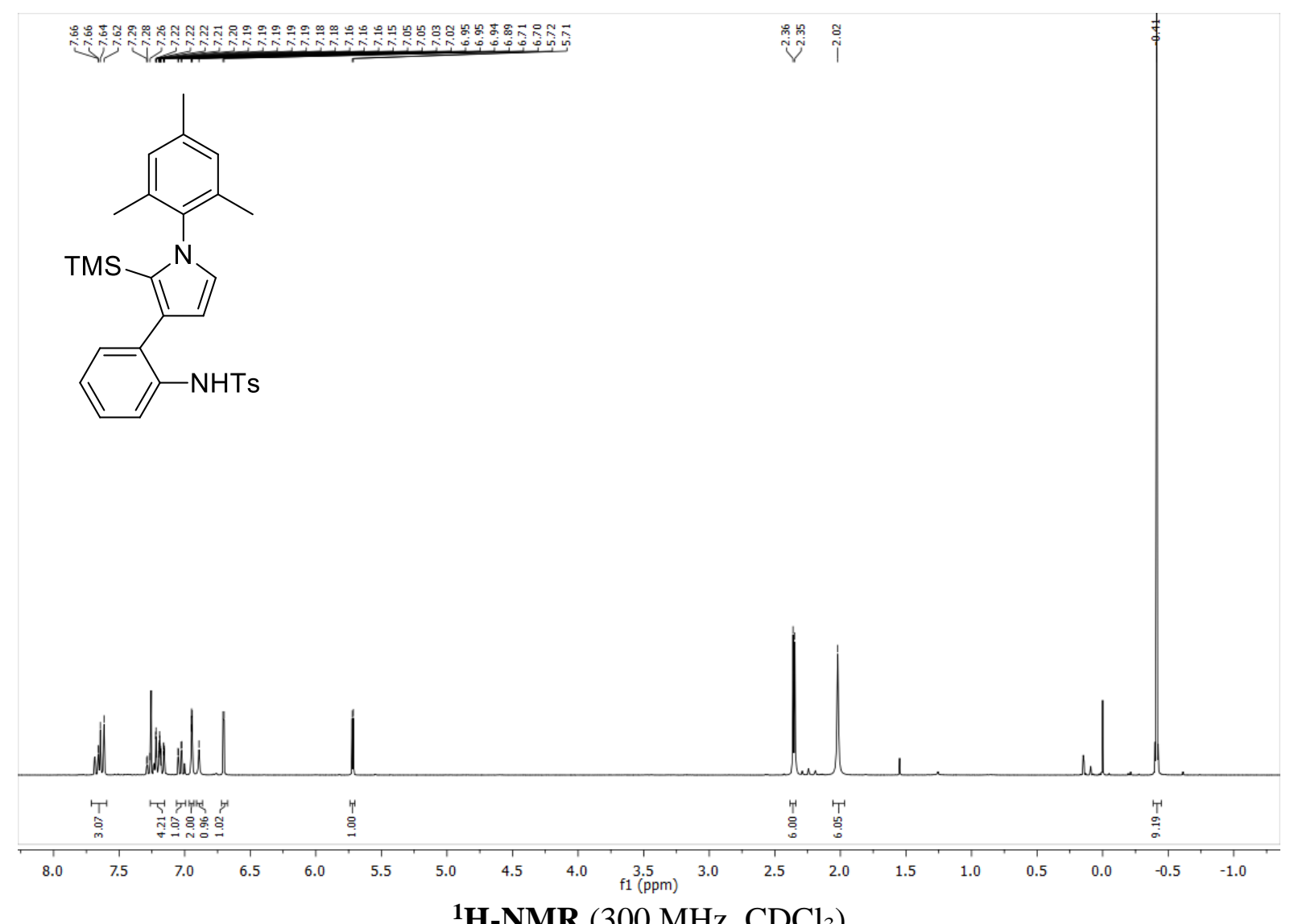

${ }^{1} \mathbf{H}-\mathbf{N M R}\left(300 \mathrm{MHz}, \mathrm{CDCl}_{3}\right)$

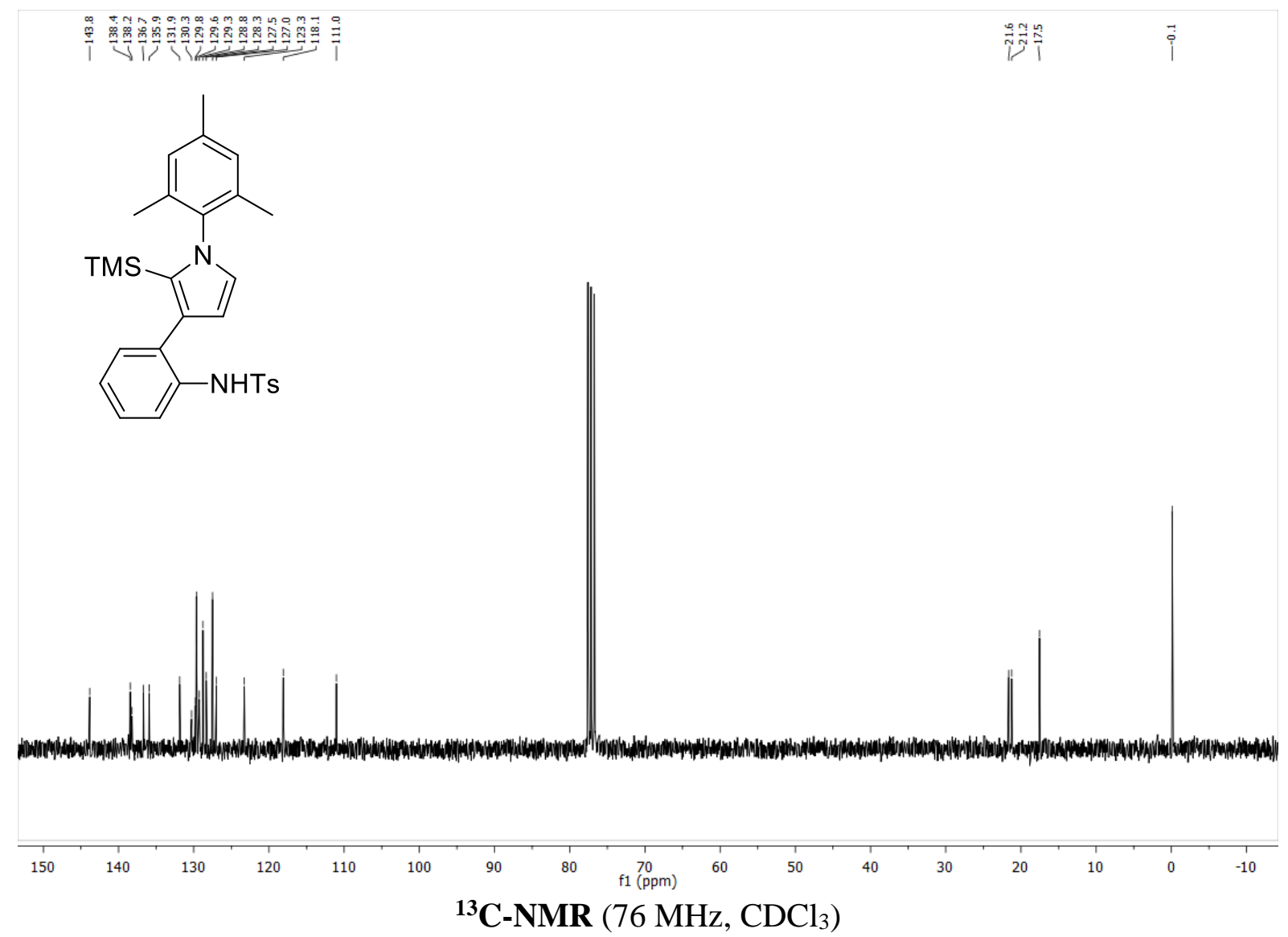


Analytical data of $\mathbf{5 f b}$ :

${ }^{1}$ H-NMR $\left(500 \mathrm{MHz}, \mathrm{CDCl}_{3}\right): \delta=2.03(\mathrm{~s}, 6 \mathrm{H}), 2.33$ (s, 3H), 2.35 (s, 3H), $6.08(\mathrm{dd}, J=2.7$, $1.8 \mathrm{~Hz}, 1 \mathrm{H}), 6.35(\mathrm{t}, J=2.0 \mathrm{~Hz}, 1 \mathrm{H}), 6.64(\mathrm{t}, J=2.4 \mathrm{~Hz}, 1 \mathrm{H}), 6.94-6.98$ (m, 2H), 7.08 (td, $J=7.4,1.2 \mathrm{~Hz}, 1 \mathrm{H}), 7.13-7.17(\mathrm{~m}, 2 \mathrm{H}), 7.19-7.25$ (m, 3H), 7.57-7.62 (m, 2H), 7.65 (dd, $J=8.2,1.2 \mathrm{~Hz}, 1 \mathrm{H})$.

${ }^{13}$ C-NMR $\left(126 \mathrm{MHz}, \mathrm{CDCl}_{3}\right) \delta=17.3,21.0,21.5,108.8,119.9,120.1,120.1,123.2,124.4$, $127.1,127.5,127.7,128.8,129.5,130.4,134.2,135.5,136.5,136.7,138.2,143.7$.

IR (ATR): $\tilde{v}\left(\mathrm{~cm}^{-1}\right)=3316(\mathrm{w}), 3029(\mathrm{w}), 2953(\mathrm{w}), 2921(\mathrm{w}), 2859(\mathrm{w}), 1496(\mathrm{~m}), 1400$ (m), $1335(\mathrm{~m}), 1161(\mathrm{~s}), 1089(\mathrm{~m})$.

ESI-HRMS: $\mathrm{C}_{26} \mathrm{H}_{26} \mathrm{~N}_{2} \mathrm{O}_{2} \mathrm{~S} m / z$ calcd. $\left[\mathrm{M}+\mathrm{H}^{+}\right]$: 431.1788 found 431.1791 
<smiles></smiles>

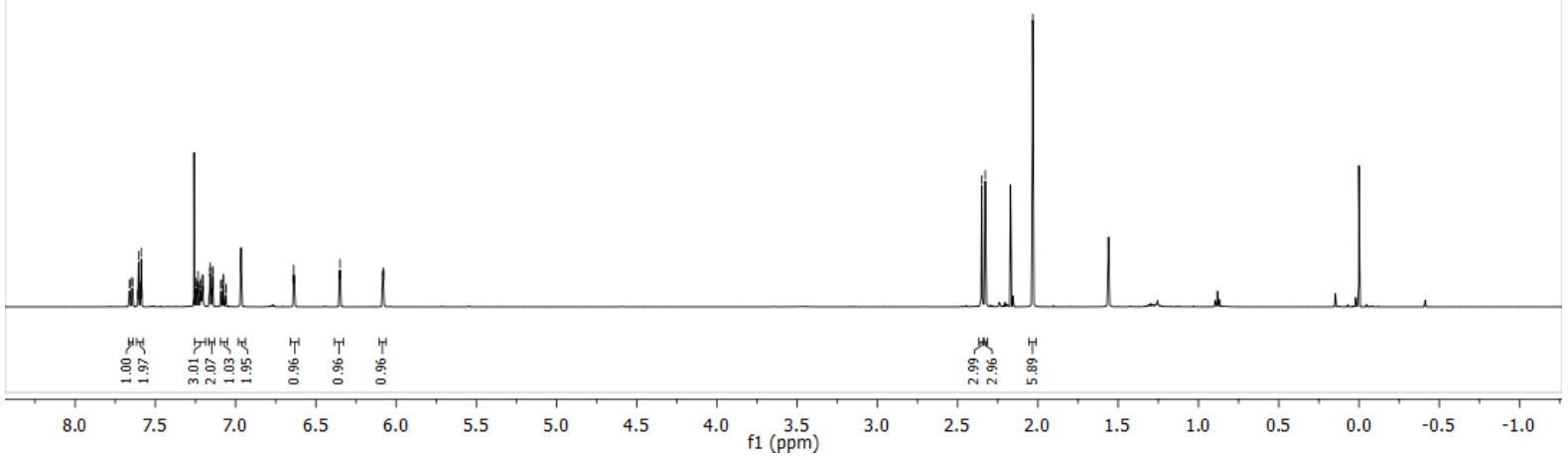

${ }^{1} \mathbf{H}-\mathbf{N M R}\left(500 \mathrm{MHz}, \mathrm{CDCl}_{3}\right)$

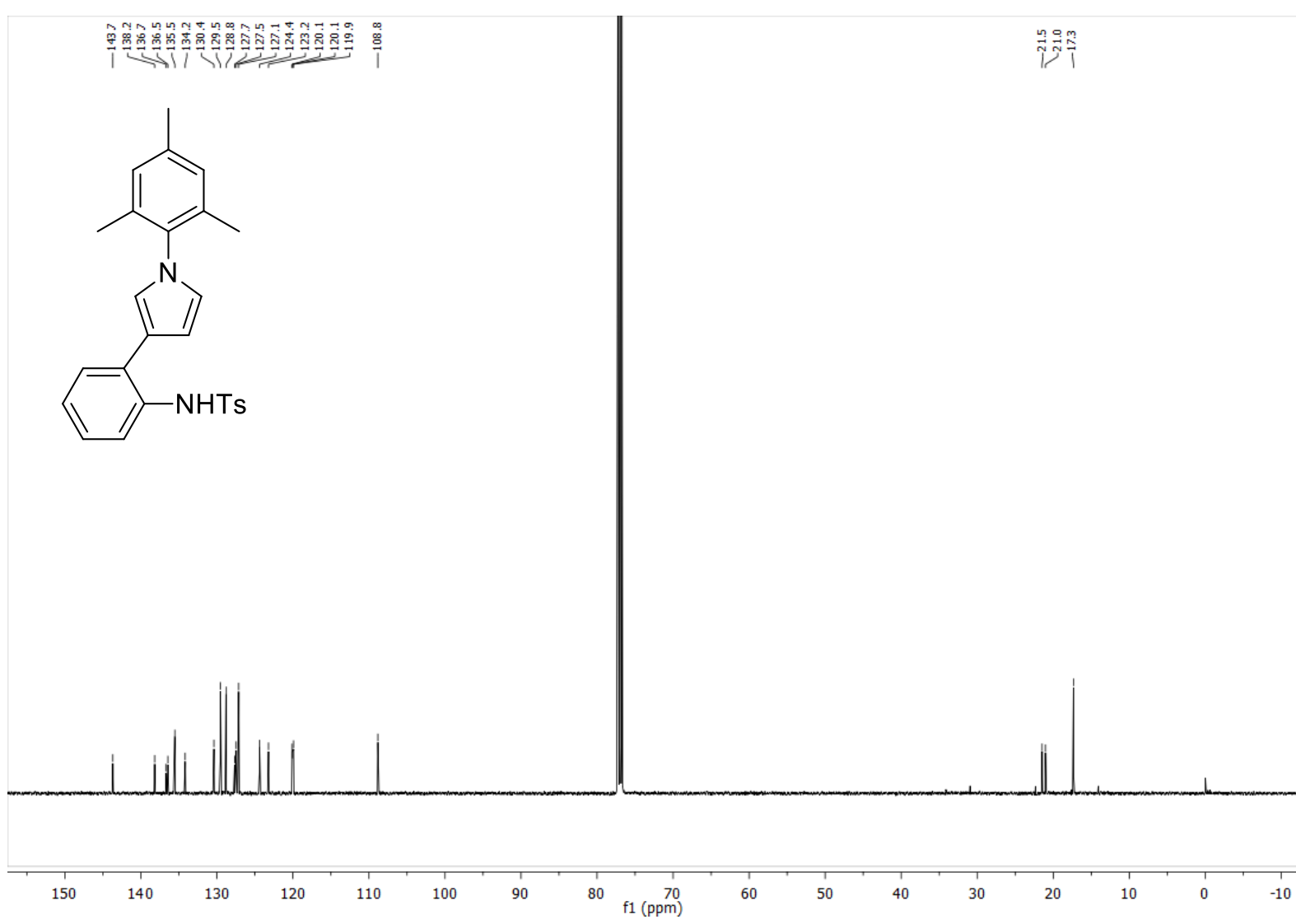

${ }^{13}$ C-NMR $\left(126 \mathrm{MHz}, \mathrm{CDCl}_{3}\right)$ 


\section{Domino product $(5 \mathrm{~g})$}

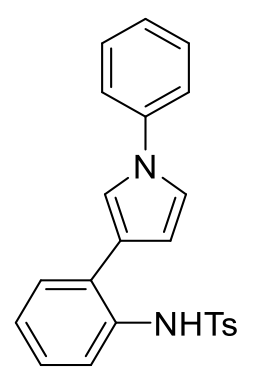

The domino precursor $\mathbf{2 g}(51.6 \mathrm{mg}, 95.3 \mu \mathrm{mol})$, triethylamine $(70 \mu \mathrm{L}, 505 \mu \mathrm{mol})$, $\left[t \mathrm{Bu}_{3} \mathrm{PH}\right]\left[\mathrm{BF}_{4}\right](2.8 \mathrm{mg}, 9.65 \mu \mathrm{mol})$ and $\mathrm{PdCl}_{2}(\mathrm{PhCN})_{2}(1.8 \mathrm{mg}, 4.69 \mu \mathrm{mol})$ were reacted according to GP3 (Pd-catalyzed Domino Reaction). The mixture was stirred $2 \mathrm{~h}$ at $120^{\circ} \mathrm{C}$. Silica gel column chromatography (EtOAc: $n$-pentane $=1: 15)$ afforded domino product $\mathbf{5 g}$ (21.6 mg, $55.6 \mu \mathrm{mol}, 58 \%)$ as yellow oil.

${ }^{1}$ H-NMR $\left(300 \mathrm{MHz}, \mathrm{CDCl}_{3}\right): \delta=2.31(\mathrm{~s}, 3 \mathrm{H}), 6.11(\mathrm{dd}, J=2.9,1.8 \mathrm{~Hz}, 1 \mathrm{H}), 6.65(\mathrm{t}$, $J=2.0 \mathrm{~Hz}, 1 \mathrm{H}), 7.04(\mathrm{~s}, 1 \mathrm{H}), 7.08-7.17(\mathrm{~m}, 4 \mathrm{H}), 7.22-7.37(\mathrm{~m}, 5 \mathrm{H}), 7.43-7.51(\mathrm{~m}, 2 \mathrm{H})$, 7.53-7.61 (m, 2H), $7.65(\mathrm{dd}, J=8.1,1.2 \mathrm{~Hz}, 1 \mathrm{H})$.

${ }^{13}$ C-NMR $\left(75 \mathrm{MHz}, \mathrm{CDCl}_{3}\right) \delta=21.5,110.7,117.9,120.5,120.5,120.9,121.7,124.7,126.2$, $127.1,127.8,127.8,129.5,129.7,130.3,134.3,136.5,140.0,143.7$.

IR (ATR): $\tilde{v}\left(\mathrm{~cm}^{-1}\right)=3320(\mathrm{w}), 3063(\mathrm{w}), 2955(\mathrm{w}), 2924(\mathrm{w}), 2857(\mathrm{w}), 1598(\mathrm{~m}), 1506$ (m), 1401 (m), 1334 (m), 1161 (s), 1090 (m).

GC-HRMS (EI): $\mathrm{C}_{23} \mathrm{H}_{20} \mathrm{~N}_{2} \mathrm{O}_{2} \mathrm{~S} m / z$ calcd. $\left[\mathrm{M}+\mathrm{H}^{+}\right]$: 389.1318 found 389.1322. 

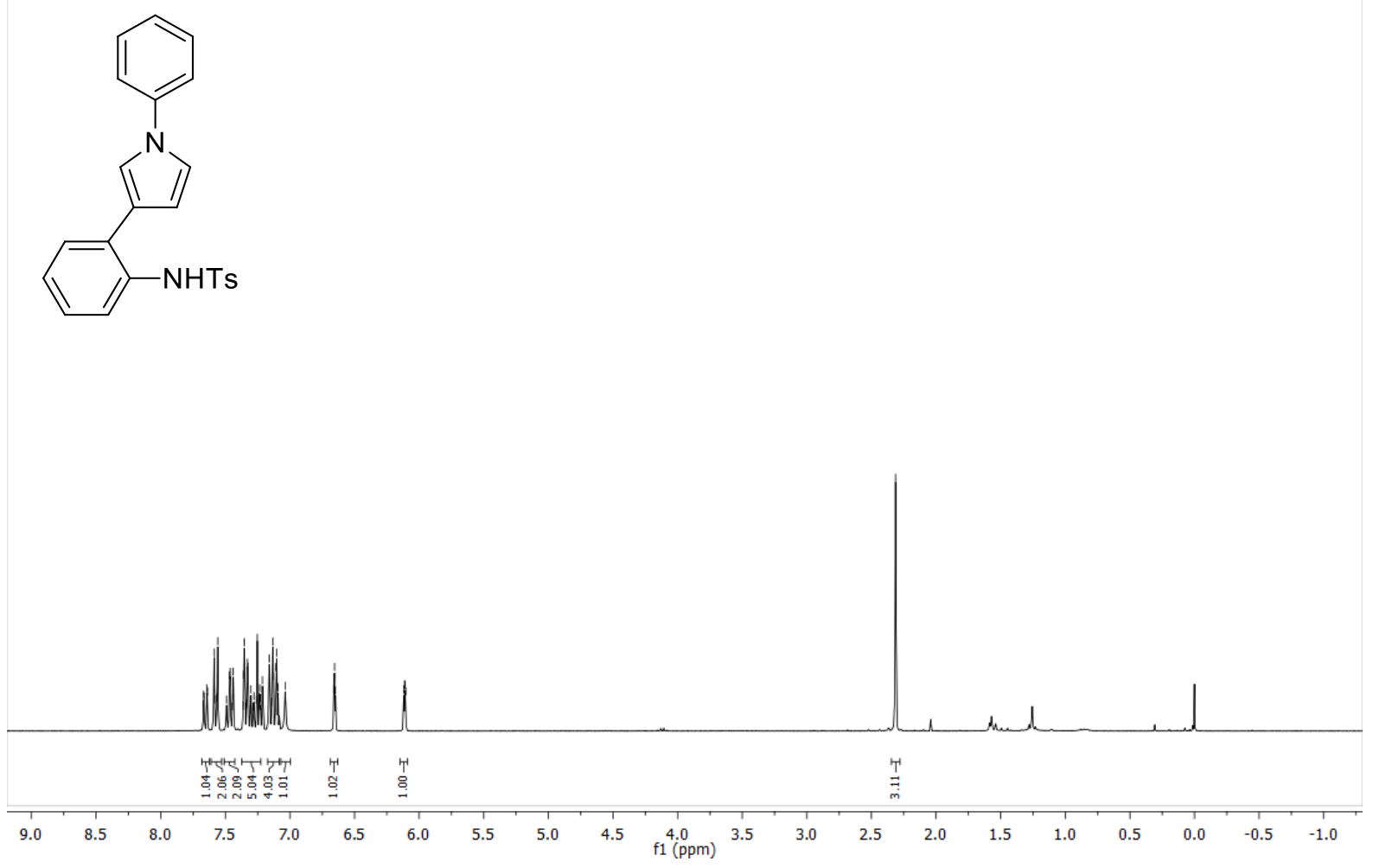

${ }^{\mathbf{1}} \mathbf{H}-\mathbf{N M R}\left(300 \mathrm{MHz}, \mathrm{CDCl}_{3}\right)$

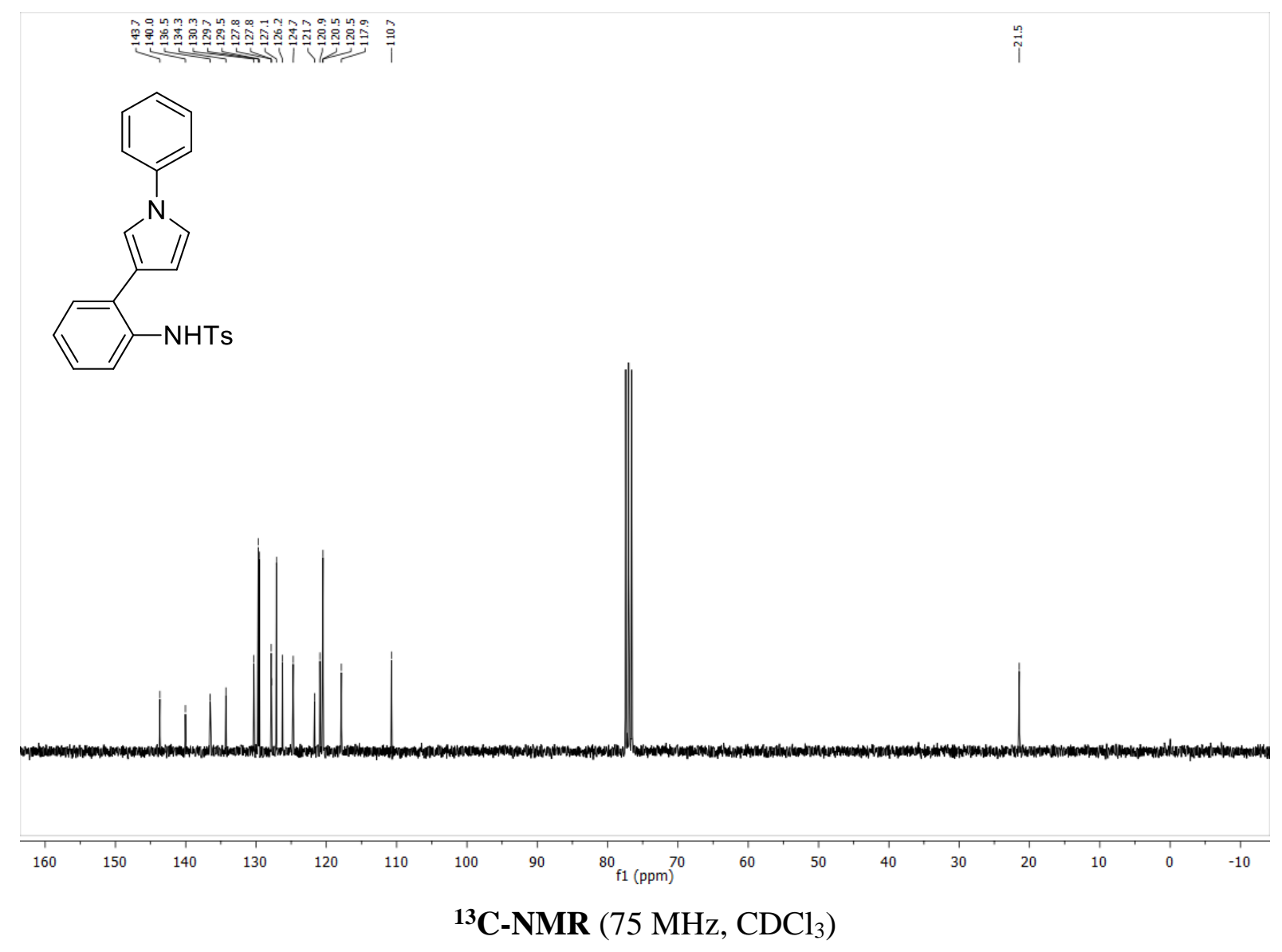




\section{Domino product $(5 \mathrm{~h})$}

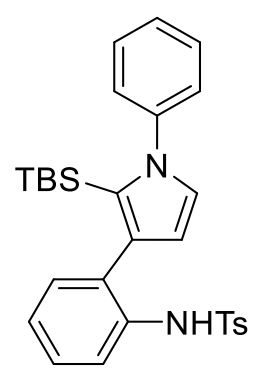

The domino precursor $\mathbf{2 h}(117 \mathrm{mg}, 200 \mu \mathrm{mol})$, triethylamine $(170 \mu \mathrm{L}, 1.23 \mathrm{mmol})$, $\left[t \mathrm{Bu}_{3} \mathrm{PH}\right]\left[\mathrm{BF}_{4}\right](6.0 \mathrm{mg}, 20.7 \mu \mathrm{mol})$ and $\mathrm{PdCl}_{2}(\mathrm{PhCN})_{2}(4.0 \mathrm{mg}, 10.4 \mu \mathrm{mol})$ were reacted according to GP3 (Pd-catalyzed Domino Reaction). The mixture was stirred $2 \mathrm{~h}$ at $120^{\circ} \mathrm{C}$. Silica gel column chromatography (EtOAc: $n$-pentane $=1: 20$ ) afforded domino product $\mathbf{5 h}$ (94.0 mg, $187 \mu \mathrm{mol}, 94 \%)$ as yellow solid.

Scale-up: The domino precursor $\mathbf{2 h}(584 \mathrm{mg}, 1.00 \mathrm{mmol})$ was dissolved in DMF $(30 \mathrm{~mL})$. To the mixture were added triethylamine $(700 \mu \mathrm{L}, 5.05 \mathrm{mmol}),\left[t \mathrm{Bu}_{3} \mathrm{PH}\right]\left[\mathrm{BF}_{4}\right](29.0 \mathrm{mg}$, $100 \mu \mathrm{mol})$ and $\mathrm{PdCl}_{2}(\mathrm{PhCN})_{2}(19.0 \mathrm{mg}, 49.5 \mu \mathrm{mol})$ in that order. The reaction flask was closed with a teflon cap and stirred $30 \mathrm{~min}$ at rt. Afterwards, the mixture was stirred $2 \mathrm{~h}$ at $120{ }^{\circ} \mathrm{C}$. The mixture was cooled to $\mathrm{rt}$ and water and EtOAc were added. The layers were separated and the aqueous layer was extracted with EtOAc (2x). The combined organic layers were dried over $\mathrm{Na}_{2} \mathrm{SO}_{4}$ and the solvent was removed in vacuo. Silica gel column chromatography (EtOAc: $n$-pentane $=1: 20)$ afforded domino product $\mathbf{5 h}(432 \mathrm{mg}, 859 \mu \mathrm{mol}$, $86 \%$ ) as yellow solid.

${ }^{1}$ H-NMR (300 MHz, $\mathrm{CDCl}_{3}$ ): $\delta=-0.49$ (s, 3H), -0.31 (s, 3H), 0.61 (s, 9H), 2.37 (s, 3H), 5.56 $(\mathrm{d}, J=2.4 \mathrm{~Hz}, 1 \mathrm{H}), 6.88-6.96(\mathrm{~m}, 2 \mathrm{H}), 6.96-7.05(\mathrm{~m}, 1 \mathrm{H}), 7.16-7.28(\mathrm{~m}, 5 \mathrm{H}), 7.38-7.47(\mathrm{~m}$, $5 \mathrm{H}), 7.64(\mathrm{dd}, J=8.4,1.7 \mathrm{~Hz}, 3 \mathrm{H})$.

${ }^{13}$ C-NMR $\left(75 \mathrm{MHz}, \mathrm{CDCl}_{3}\right) \delta=-3.9,-1.9,18.3,21.5,27.7,111.4,117.7,122.8,127.4$, 128.2, 128.3, 128.4, 128.5, 129.1, 129.5, 129.5, 131.8, 132.1, 135.9, 136.4, 142.5, 143.7.

IR (ATR): $\tilde{v}\left(\mathrm{~cm}^{-1}\right)=3308(\mathrm{w}), 2954(\mathrm{~m}), 2928(\mathrm{~m}), 2892(\mathrm{w}), 2855(\mathrm{w}), 1497(\mathrm{~m}), 1394$ (m), $1334(\mathrm{~m}), 1164(\mathrm{~s}), 1092(\mathrm{~m})$.

M.p.: $127 \neg 130{ }^{\circ} \mathrm{C}$.

ESI-HRMS: $\mathrm{C}_{29} \mathrm{H}_{34} \mathrm{~N}_{2} \mathrm{O}_{2} \mathrm{SSi} m / z$ calcd. $\left[\mathrm{M}+\mathrm{H}^{+}\right]: 503.2183$ found 503.2182. 


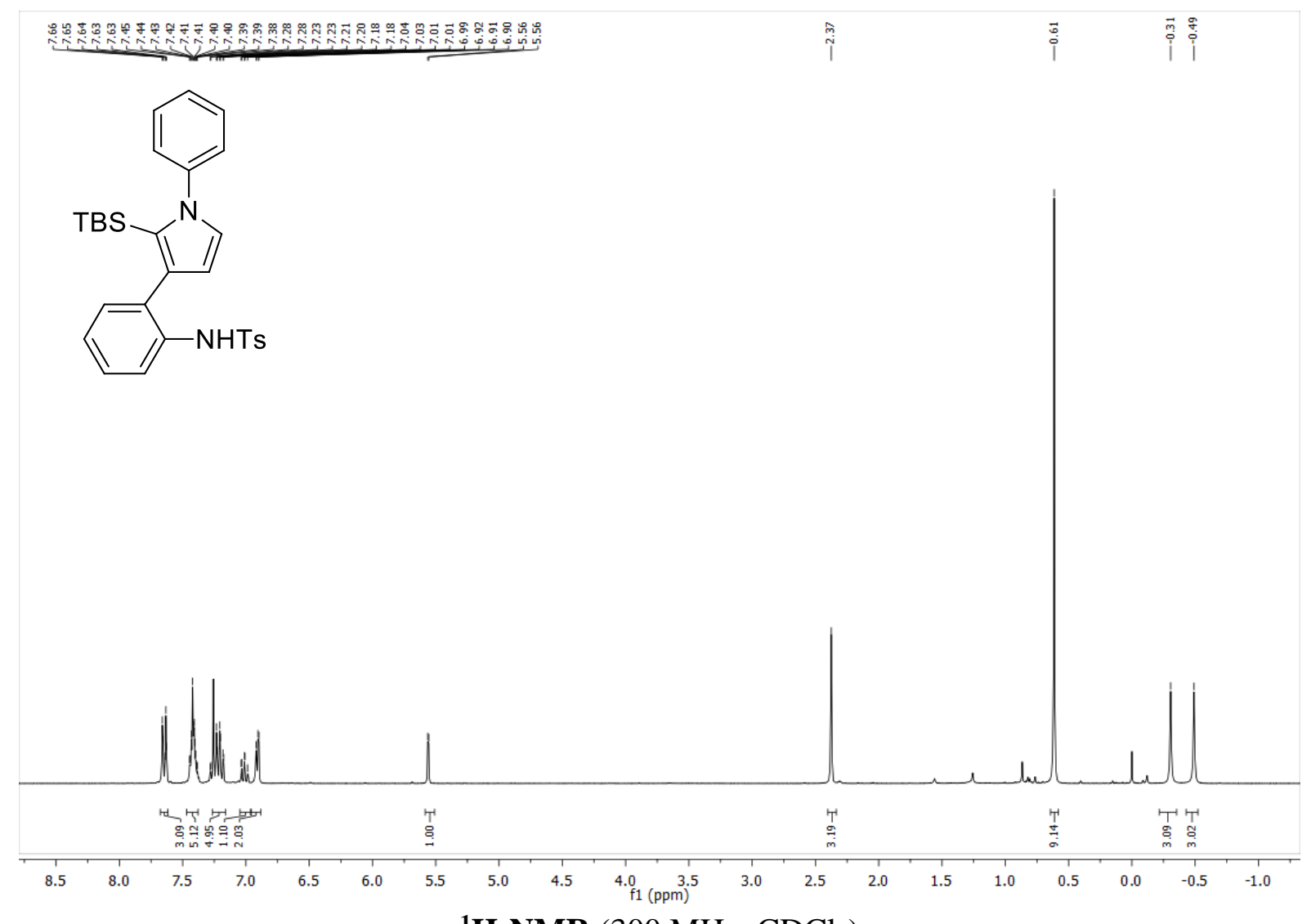

${ }^{\mathbf{1}} \mathbf{H}-\mathbf{N M R}\left(300 \mathrm{MHz}, \mathrm{CDCl}_{3}\right.$ )

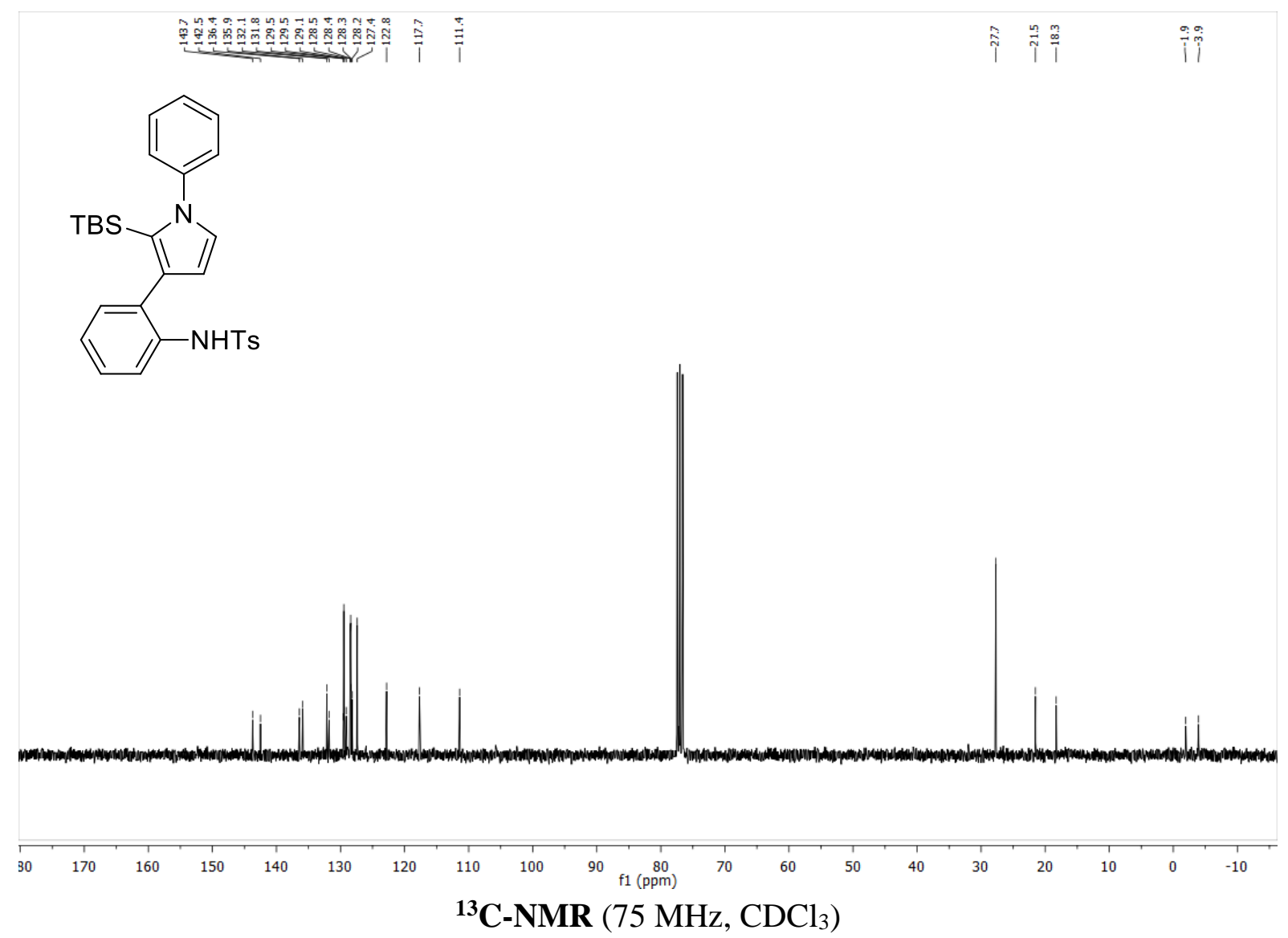


7) Termination with different Amines
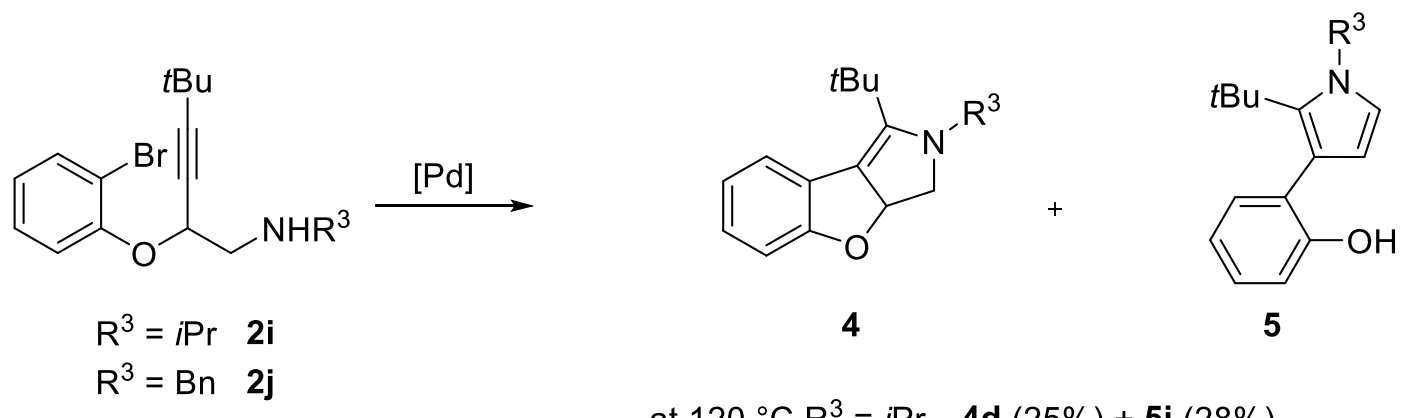

$\begin{array}{ll}\text { at } 120{ }^{\circ} \mathrm{C} \mathrm{R} \mathrm{R}^{3}=\operatorname{iPr} & \mathbf{4 d}(25 \%)+\mathbf{5 i}(28 \%) \\ \text { at } 100{ }^{\circ} \mathrm{C} \mathrm{R}^{3}=i \operatorname{Pr} & \mathbf{4 d}(47 \%)+\mathbf{5 i}(12 \%) \\ \text { at } 100{ }^{\circ} \mathrm{C} \mathrm{R}^{3}=\operatorname{Bn} & \mathbf{4 e}(46 \%)+\mathbf{5 j} \text { (traces) }\end{array}$ 


\section{Domino product 4d and Domino product $5 i$}<smiles>CC(C)N1CC2Oc3ccccc3C2=C1C(C)(C)C</smiles>

4d

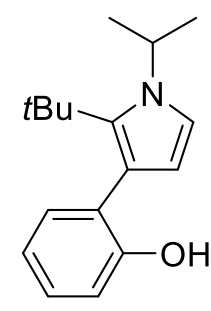

$5 \mathbf{i}$

The domino precursor $2 \mathbf{i}(32.8 \mathrm{mg}, 97.0 \mu \mathrm{mol})$, potassium phosphate $(105 \mathrm{mg}, 505 \mu \mathrm{mol})$, $\left[t \mathrm{Bu}_{3} \mathrm{PH}\right]\left[\mathrm{BF}_{4}\right](3.2 \mathrm{mg}, 11.0 \mu \mathrm{mol})$ and $\mathrm{PdCl}_{2}(\mathrm{PhCN})_{2}(2.1 \mathrm{mg}, 5.5 \mu \mathrm{mol})$ were reacted according to GP3 (Pd-catalyzed Domino Reaction). The mixture was stirred $2 \mathrm{~h}$ at $120^{\circ} \mathrm{C}$. Silica gel column chromatography (EtOAc: $n$-pentane $=1: 20$ ) afforded a mixture of domino product $\mathbf{4 d}(7.1 \mathrm{mg}, 27.6 \mu \mathrm{mol}, 28 \%)$ as yellow oil and domino product $\mathbf{5 i}(6.3 \mathrm{mg}$, $24.5 \mu \mathrm{mol}, 25 \%)$ as yellow oil.

The domino precursor $2 \mathbf{i}(33.7 \mathrm{mg}, 99.6 \mu \mathrm{mol})$, potassium phosphate (107 mg, $504 \mu \mathrm{mol})$, $\left[t \mathrm{Bu}_{3} \mathrm{PH}\right]\left[\mathrm{BF}_{4}\right](3.5 \mathrm{mg}, 12.0 \mu \mathrm{mol})$ and $\mathrm{PdCl}_{2}(\mathrm{PhCN})_{2}(1.9 \mathrm{mg}, 5.0 \mu \mathrm{mol})$ were reacted according to GP3 (Pd-catalyzed Domino Reaction). The mixture was stirred $2 \mathrm{~h}$ at $100{ }^{\circ} \mathrm{C}$. Silica gel column chromatography (EtOAc: $n$-pentane $=1: 20)$ afforded a mixture of domino product $4 \mathbf{d}(12.0 \mathrm{mg}, 46.6 \mu \mathrm{mol}, 47 \%)$ as yellow oil and domino product $\mathbf{5 i}(3.0 \mathrm{mg}$, $11.7 \mu \mathrm{mol}, 12 \%)$ as yellow oil.

Analytical data for $\mathbf{4 d}$ :

${ }^{1}$ H-NMR (300 MHz, $\left.\mathrm{CDCl}_{3}\right): \delta=1.15(\mathrm{~d}, J=6.6 \mathrm{~Hz}, 3 \mathrm{H}), 1.21(\mathrm{~d}, J=6.6 \mathrm{~Hz}, 3 \mathrm{H}), 1.24$ (s, 9H), $3.11(\mathrm{dd}, J=12.0,8.0 \mathrm{~Hz}, 1 \mathrm{H}), 3.39$ (dd, $J=12.1,2.6 \mathrm{~Hz}, 1 \mathrm{H}), 4.08$ (p, $J=6.6 \mathrm{~Hz}, 1 \mathrm{H})$, $4.76(\mathrm{dd}, J=8.0,2.6 \mathrm{~Hz}, 1 \mathrm{H}), 6.59$ (td, $J=7.5,1.6 \mathrm{~Hz}, 1 \mathrm{H}), 6.70-6.77$ (m, 1H), 6.80-6.89 $(\mathrm{m}, 2 \mathrm{H})$.

${ }^{13}$ C-NMR $\left(76 \mathrm{MHz}, \mathrm{CDCl}_{3}\right) \delta=17.9,19.4,27.4,30.9,44.3,46.6,64.9,74.9,95.6,111.9$, 117.0, 117.0, 121.8, 134.2, 143.4.

IR (ATR): $\tilde{v}\left(\mathrm{~cm}^{-1}\right)=2970(\mathrm{~m}), 2929(\mathrm{w}), 2870(\mathrm{w}), 1605(\mathrm{w}), 1500(\mathrm{~s}), 1461(\mathrm{w}), 1333(\mathrm{~m})$, 1264 (s), 1204 (m), 1081 (m).

GC-HRMS (EI): $\mathrm{C}_{17} \mathrm{H}_{23} \mathrm{NO} \mathrm{m} / \mathrm{z}$ calcd. $\left[\mathrm{M}^{+}\right]: 257.1780$ found 257.1790. 


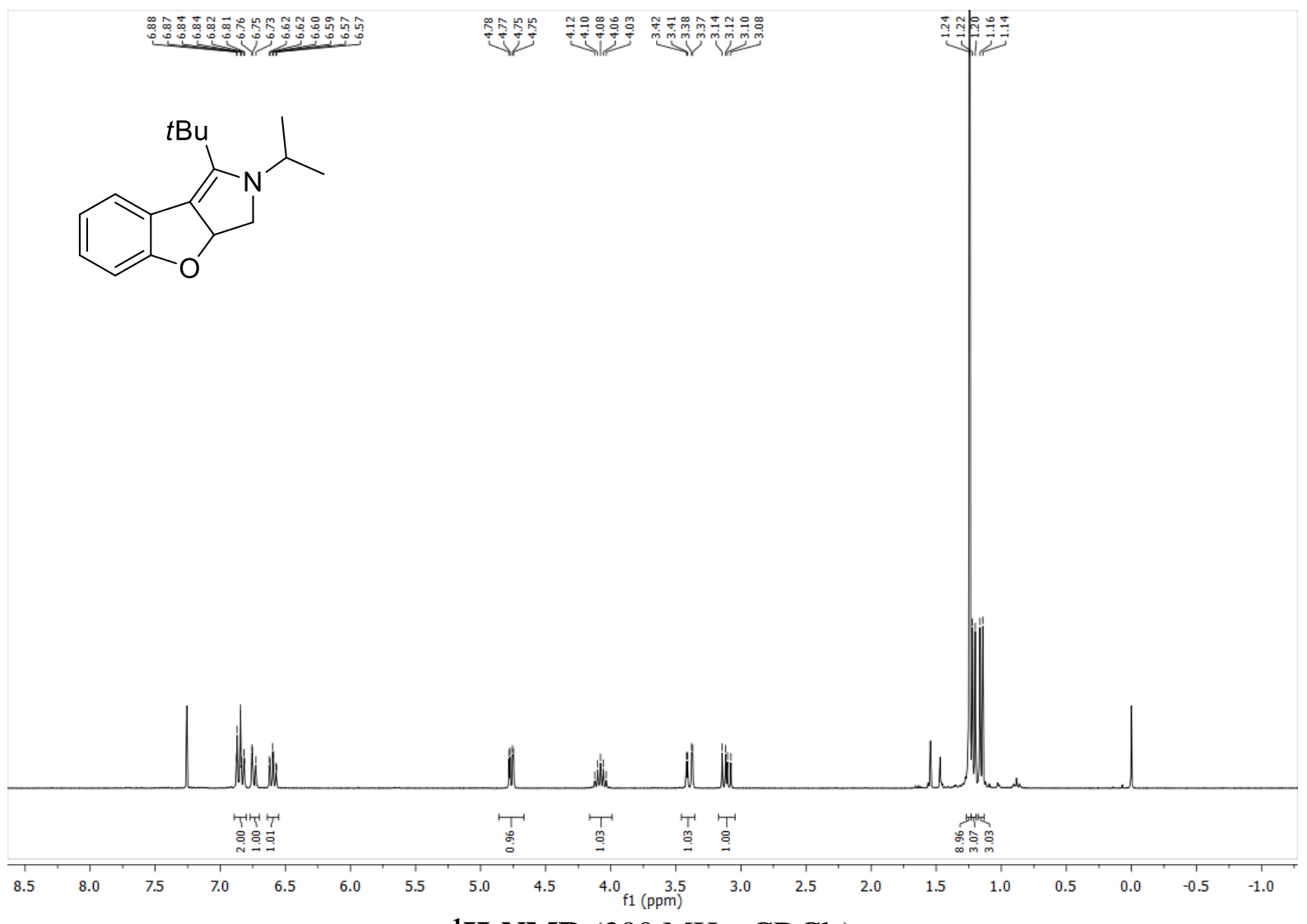

${ }^{1}$ H-NMR (300 MHz, $\mathrm{CDCl}_{3}$ )

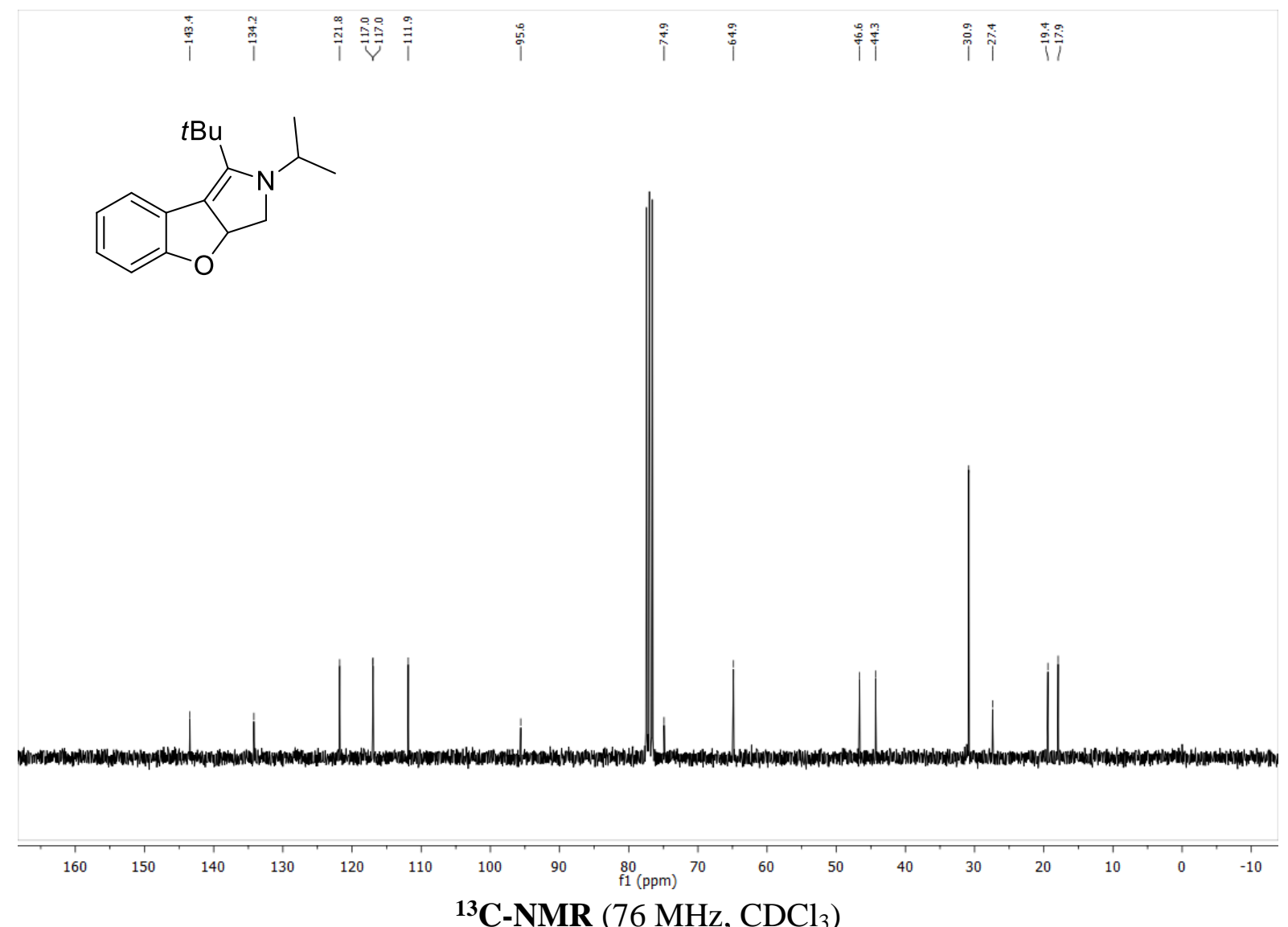

${ }^{13} \mathbf{C}$-NMR $\left(76 \mathrm{MHz}, \mathrm{CDCl}_{3}\right)$ 
Analytical data for 5i:

${ }^{\mathbf{1}} \mathbf{H}-\mathrm{NMR}\left(400 \mathrm{MHz}, \mathrm{CDCl}_{3}\right): \delta=1.24(\mathrm{~s}, 9 \mathrm{H}), 1.49(\mathrm{dd}, J=6.6,5.9 \mathrm{~Hz}, 6 \mathrm{H}), 4.79(\mathrm{p}$, $J=6.7 \mathrm{~Hz}, 1 \mathrm{H}), 5.22(\mathrm{~s}, 1 \mathrm{H}), 5.93(\mathrm{~d}, J=3.0 \mathrm{~Hz}, 1 \mathrm{H}), 6.80(\mathrm{~d}, J=3.0 \mathrm{~Hz}, 1 \mathrm{H}), 6.82-6.92$ $(\mathrm{m}, 2 \mathrm{H}), 7.13-7.20(\mathrm{~m}, 2 \mathrm{H})$.

${ }^{13}$ C-NMR $\left(101 \mathrm{MHz}, \mathrm{CDCl}_{3}\right) \delta=24.4,24.4,31.6,33.6,48.3,111.1,111.8,113.7,117.8$, $119.3,127.4,128.1,131.5,136.6,153.8$.

IR (ATR): $\tilde{v}\left(\mathrm{~cm}^{-1}\right)=3488(\mathrm{~m}), 3035(\mathrm{w}), 2972(\mathrm{~m}), 2875(\mathrm{w}), 1578(\mathrm{w}), 1501(\mathrm{~m}), 1465$ (m), $1243(\mathrm{~m}), 1191(\mathrm{~s})$.

GC-HRMS (EI): $\mathrm{C}_{17} \mathrm{H}_{23} \mathrm{NO} \mathrm{m} / \mathrm{z}$ calcd. $\left[\mathrm{M}^{+}\right]$: 257.1180 found 257.1783 . 


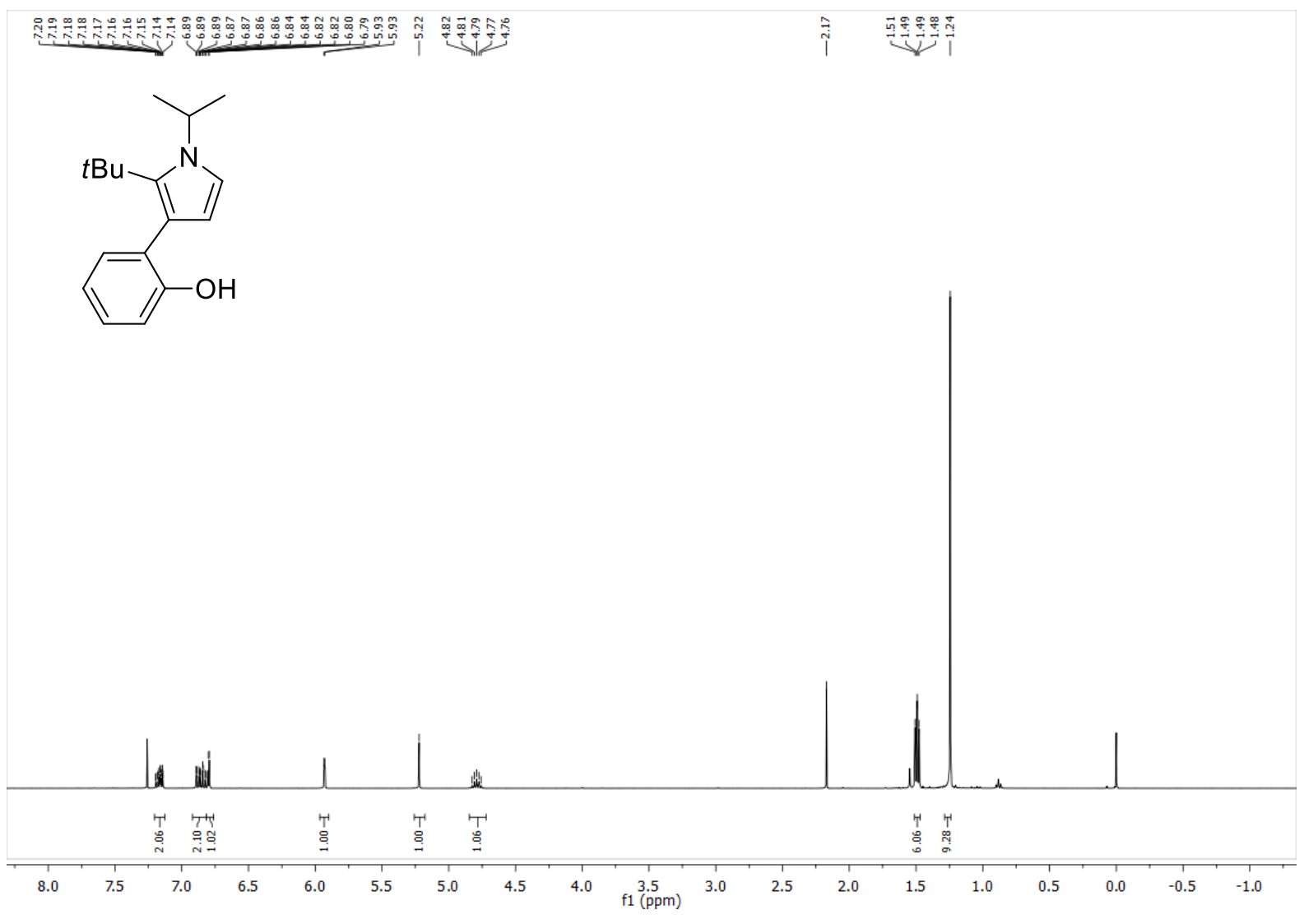

${ }^{1} \mathbf{H}-\mathbf{N M R}\left(400 \mathrm{MHz}, \mathrm{CDCl}_{3}\right)$

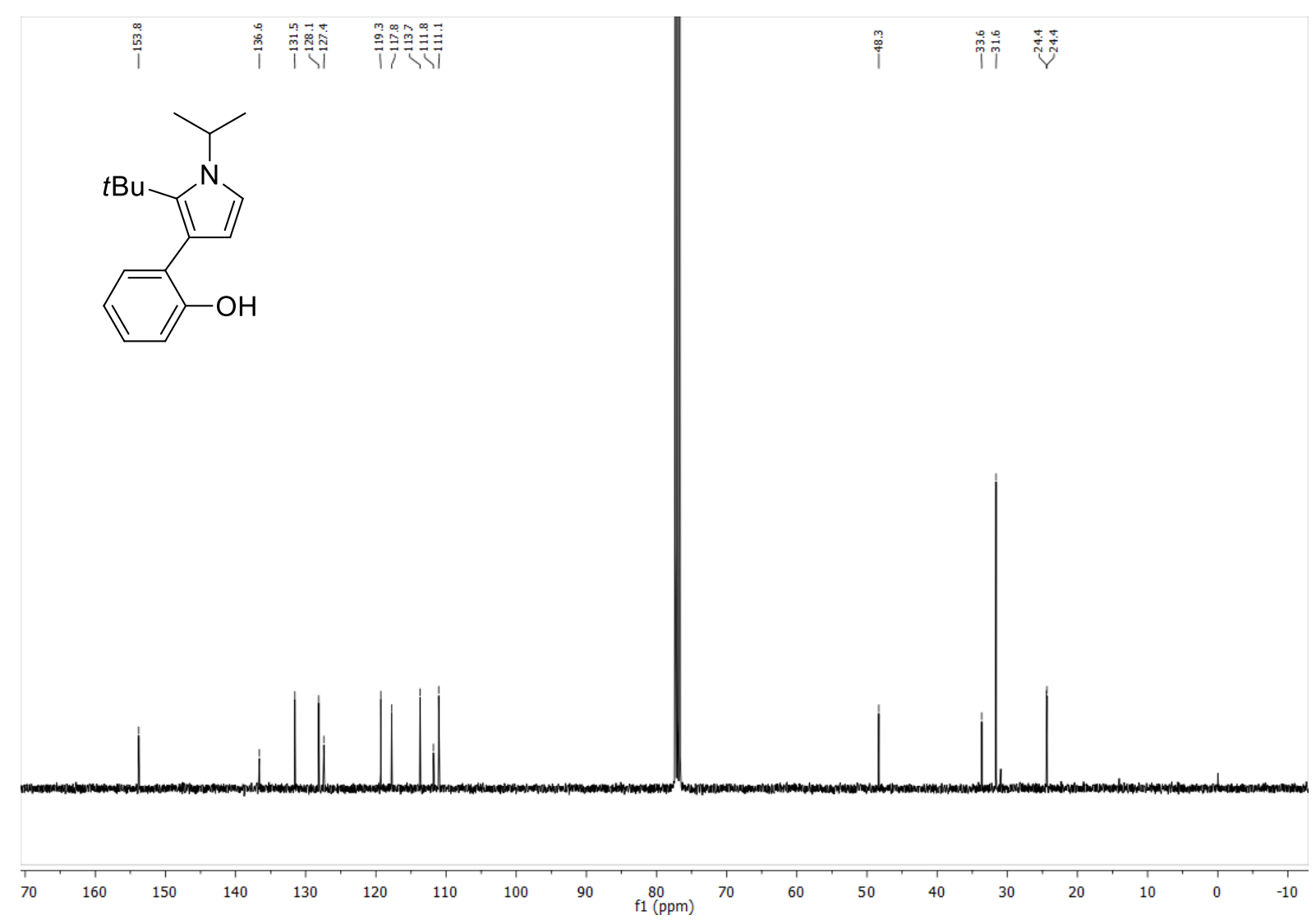

${ }^{13}$ C-NMR (101 MHz, $\left.\mathrm{CDCl}_{3}\right)$ 


\section{Domino product 4e and Domino product 5j}<smiles>CC(C)(C)C1=C2c3ccccc3OC2CN1Cc1ccccc1</smiles>

$4 e$

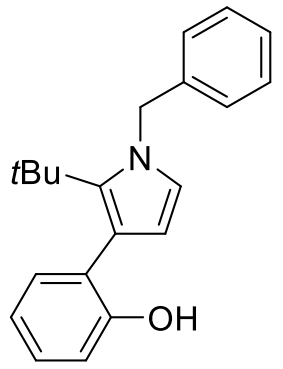

$5 \mathbf{j}$

The domino precursor $\mathbf{2 j}$ (37.6 mg, $97.3 \mu \mathrm{mol})$, potassium phosphate (104 mg, $490 \mu \mathrm{mol})$, $\left[t \mathrm{Bu}_{3} \mathrm{PH}\right]\left[\mathrm{BF}_{4}\right](2.5 \mathrm{mg}, 8.6 \mu \mathrm{mol})$ and $\mathrm{PdCl}_{2}(\mathrm{PhCN})_{2}(2.3 \mathrm{mg}, 6.0 \mu \mathrm{mol})$ were reacted according to GP3 (Pd-catalyzed Domino Reaction). The mixture was stirred $2 \mathrm{~h}$ at $100{ }^{\circ} \mathrm{C}$. Silica gel column chromatography (EtOAc: $n$-pentane $=1: 20$ ) afforded domino product $\mathbf{4 e}$ (13.6 $\mathrm{mg}, 44.6 \mu \mathrm{mol}, 46 \%)$ as yellow oil and domino product $\mathbf{5 j}$ only in traces.

Analytical data for $\mathbf{4 e}$ :

${ }^{1}$ H-NMR (300 MHz, $\left.\mathrm{CDCl}_{3}\right): \delta=1.22(\mathrm{~s}, 9 \mathrm{H}), 3.34-3.48(\mathrm{~m}, 2 \mathrm{H}), 4.37-4.57(\mathrm{~m}, 2 \mathrm{H}), 4.88$ $(\mathrm{dd}, J=6.6,3.7 \mathrm{~Hz}, 1 \mathrm{H}), 6.60-6.70(\mathrm{~m}, 2 \mathrm{H}), 6.79(\mathrm{ddd}, J=8.2,7.2,1.6 \mathrm{~Hz}, 1 \mathrm{H}), 6.89$ (dd, $J=8.1,1.6 \mathrm{~Hz}, 1 \mathrm{H}), 7.26-7.38(\mathrm{~m}, 5 \mathrm{H})$.

${ }^{13}$ C-NMR $\left(76 \mathrm{MHz} \mathrm{CDCl}_{3}\right) \delta=27.4,30.8,52.7,55.1,64.4,74.6,95.8,112.5,116.9,117.9$, $121.9,127.0,127.2,128.7,134.7,138.1,143.0$.

IR (ATR): $\tilde{v}\left(\mathrm{~cm}^{-1}\right)=2969(\mathrm{~m}), 2926(\mathrm{w}), 2901(\mathrm{w}), 2866(\mathrm{w}), 1503(\mathrm{~s}), 1357(\mathrm{~m}), 1266(\mathrm{~m})$, 1212 (m), 1050 (w).

GC-HRMS (EI): $\mathrm{C}_{21} \mathrm{H}_{23} \mathrm{NO} \mathrm{m} / \mathrm{z}$ calcd. $\left[\mathrm{M}^{+}\right]$: 305.1780 found 305.1793. 


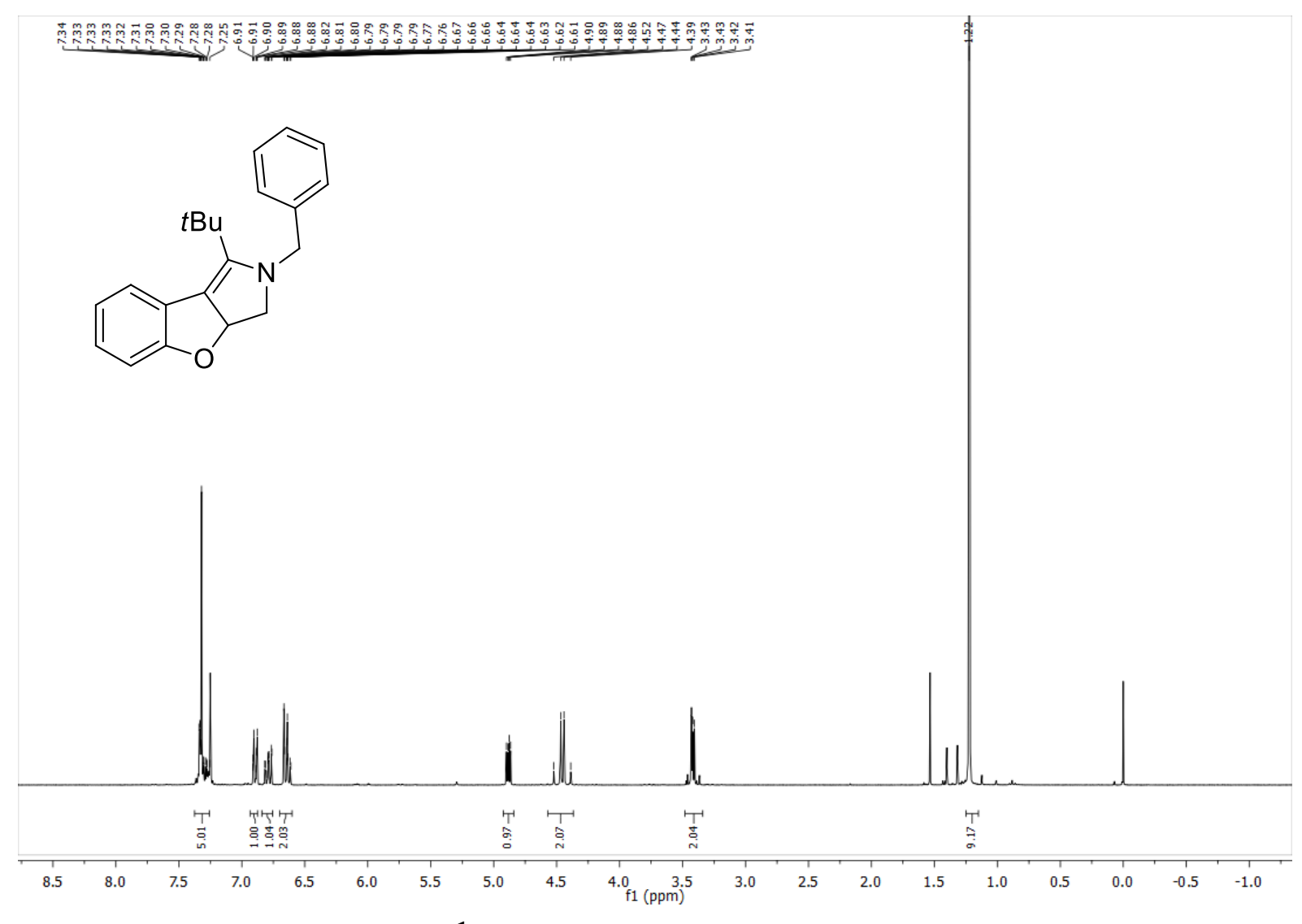

${ }^{1} \mathbf{H}-\mathbf{N M R}$ (400 MHz, $\mathrm{CDCl}_{3}$ )

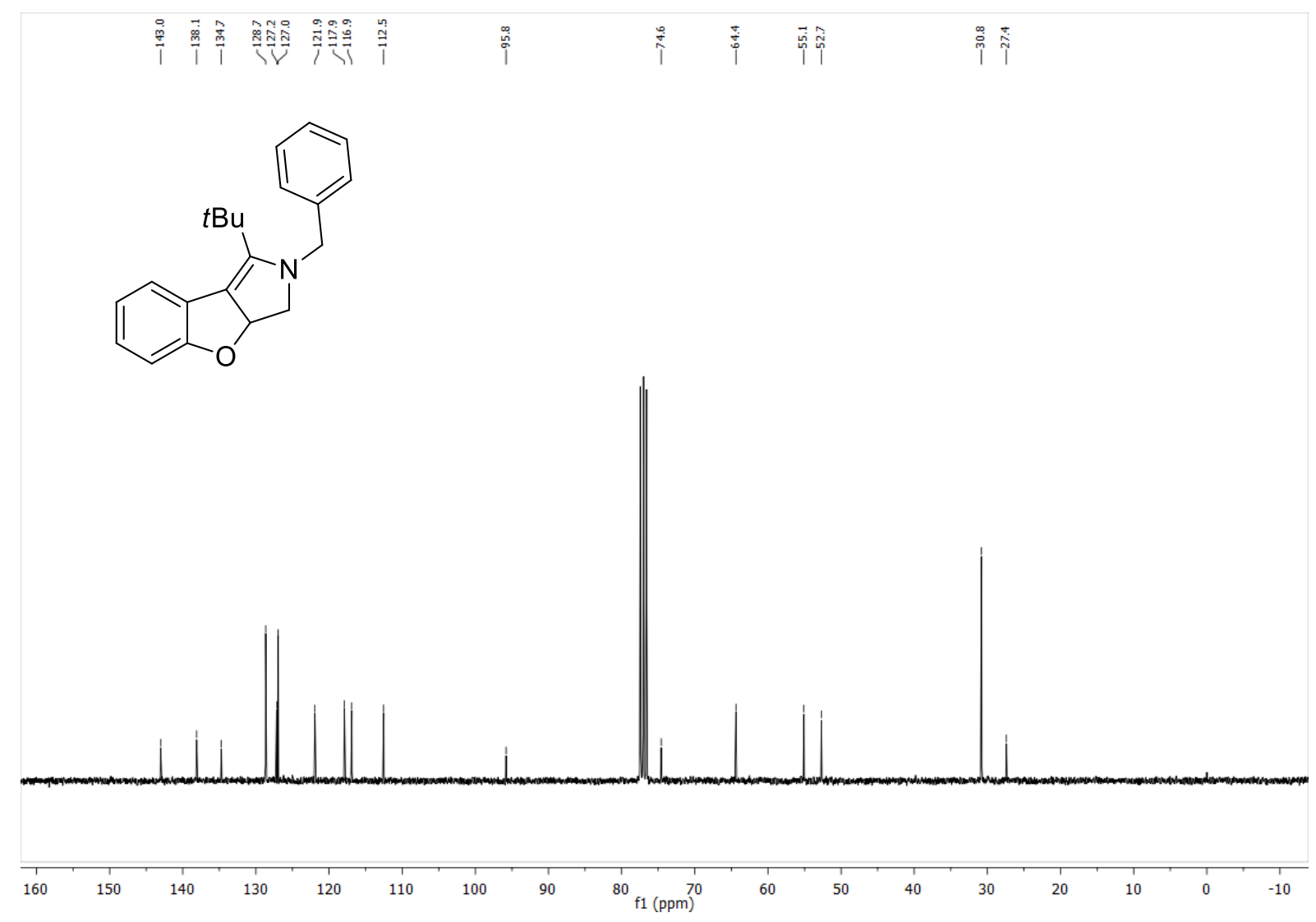

${ }^{13}$ C-NMR (101 MHz, $\left.\mathrm{CDCl}_{3}\right)$ 


\section{8) Follow-up chemistry}

$N$-(2-(2-(tert-Butyldimethylsilyl)-4,5-diiodo-1-phenyl-1H-pyrrol-3-yl)phenyl)-4-methylbenzenesulfonamide (6)

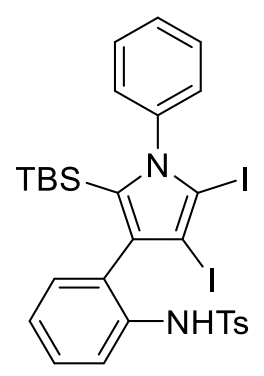

The pyrrole $5 \mathbf{h}(13.2 \mathrm{mg}, 26.3 \mu \mathrm{mol})$ was dissolved in $\mathrm{CH}_{2} \mathrm{Cl}_{2}(500 \mu \mathrm{L})$. NIS $(12.0 \mathrm{mg}$, $53.3 \mu \mathrm{mol}$ ) was added at $0{ }^{\circ} \mathrm{C}$ and the reaction mixture was stirred at ambient temperature overnight. To the reaction mixture was added silica gel and the solvent was evaporated in vасио. Silica gel column chromatography (EtOAc: $n$-pentane =1:10) afforded compound 6 (15.6 mg, $20.7 \mu \mathrm{mol}, 79 \%$ ) as colorless solid.

${ }^{1} \mathbf{H}-\mathrm{NMR}\left(500 \mathrm{MHz}, \mathrm{CH}_{2} \mathrm{Cl}_{2}\right): \delta=-0.59$ (s, 3H), -0.53 (s, 3H), 0.59 (s, 9H), $2.31(\mathrm{~s}, 3 \mathrm{H})$, $6.62(\mathrm{~s}, 1 \mathrm{H}), 6.95(\mathrm{td}, J=7.4,1.1 \mathrm{~Hz}, 1 \mathrm{H}), 7.03(\mathrm{dd}, J=7.5,1.6 \mathrm{~Hz}, 1 \mathrm{H}), 7.18-7.28(\mathrm{~m}, 5 \mathrm{H})$, 7.39-7.46 (m, 4H), 7.73-7.78 (m, 2H).

${ }^{13}$ C-NMR $\left(126 \mathrm{MHz}, \mathrm{CH}_{2} \mathrm{Cl}_{2}\right) \delta=21.7,28.0,84.4,93.2,115.7,122.5,127.4,128.0,128.8$, 129.0, 129.5, 130.1, 130.2, 130.8, 131.1, 133.7, 136.0, 136.8, 136.9, 137.0, 143.5, 144.7.

IR (ATR): $\tilde{v}\left(\mathrm{~cm}^{-1}\right)=3256(\mathrm{w}), 2930(\mathrm{w}), 2854(\mathrm{w}), 1592(\mathrm{w}), 1385(\mathrm{w}), 1332(\mathrm{~m}), 1253$ (w), $1161(\mathrm{~m}), 1090(\mathrm{~m})$.

M.p.: $210 \neg 212{ }^{\circ} \mathrm{C}$.

ESI-HRMS: $\mathrm{C}_{29} \mathrm{H}_{32} \mathrm{I}_{2} \mathrm{~N}_{2} \mathrm{O}_{2} \mathrm{SSi} / z$ calcd. $\left[\mathrm{M}+\mathrm{H}^{+}\right]$: 755.0116 found 755.0120 . 


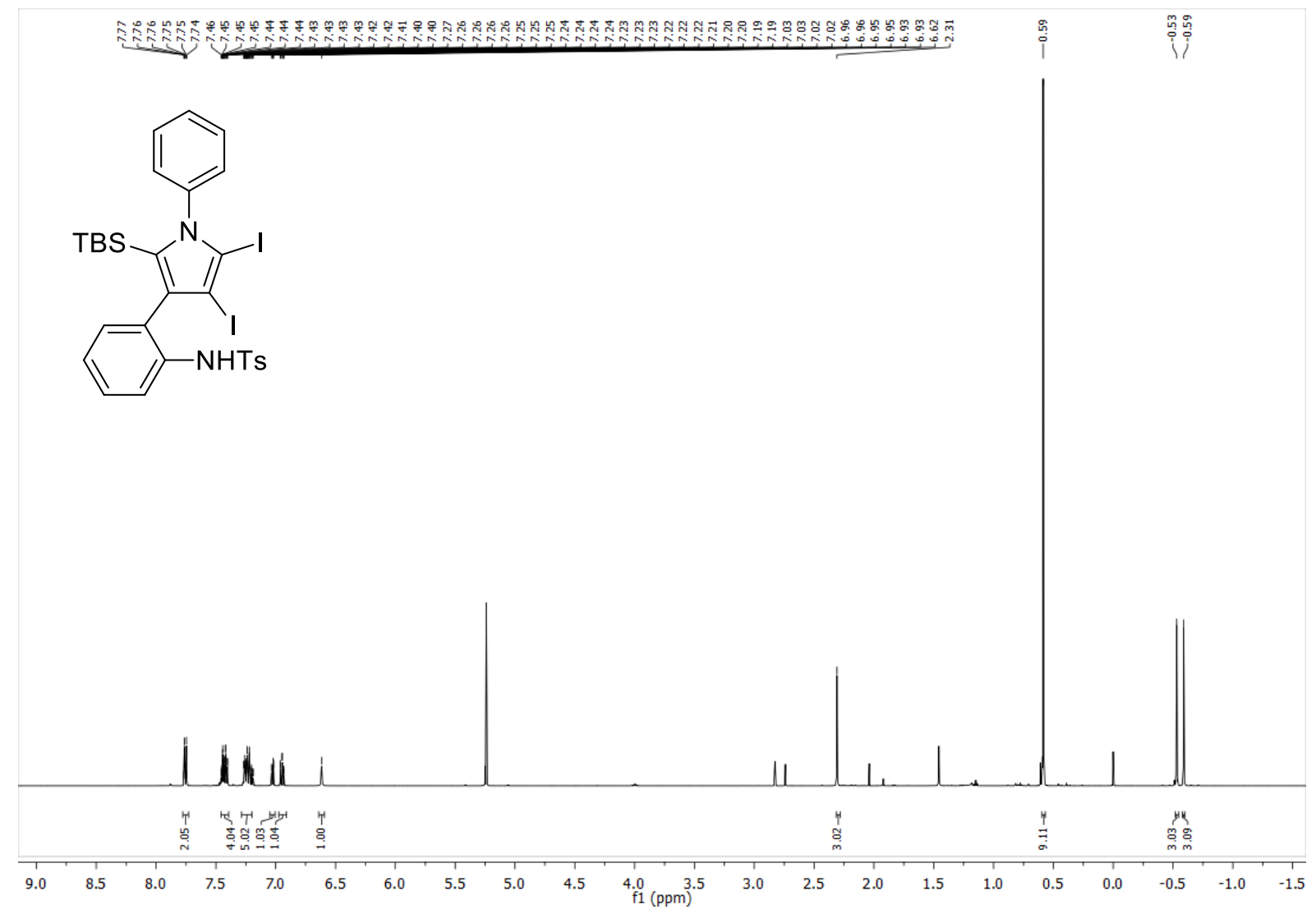

${ }^{1} \mathbf{H}$-NMR $\left(500 \mathrm{MHz}, \mathrm{CH}_{2} \mathrm{Cl}_{2}\right)$

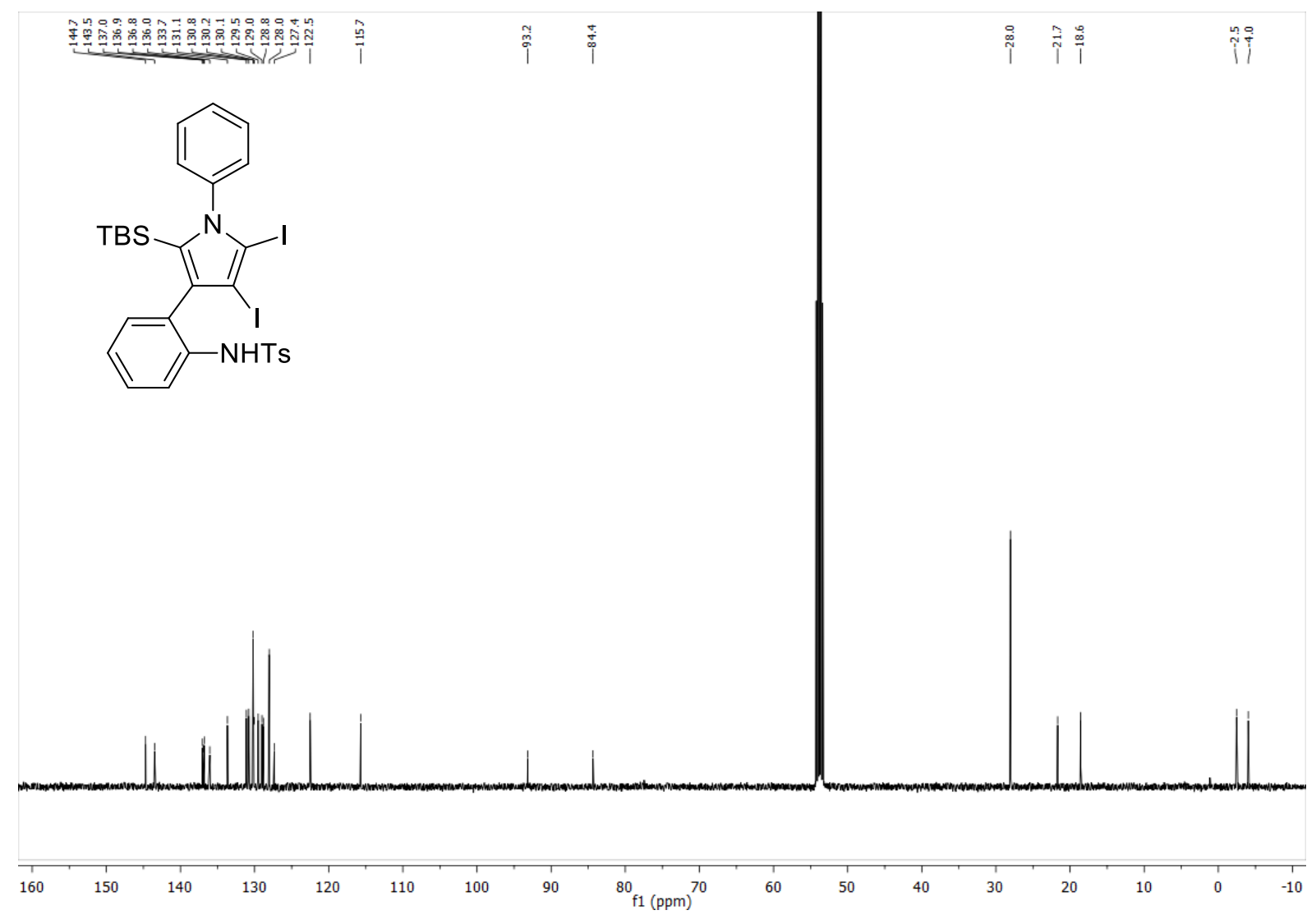

${ }^{13}$ C-NMR $\left(126 \mathrm{MHz}, \mathrm{CH}_{2} \mathrm{Cl}_{2}\right)$ 


\section{$N$-(2-(4,5-Dibromo-2-(tert-butyldimethylsilyl)-1-phenyl-1H-pyrrol-3-yl)phenyl)-4-}

methylbenzenesulfonamide (7)<smiles>CC(c1c(-c2ccccc2N)c(Br)c(Br)n1-c1ccccc1)C(F)(F)F</smiles>

The pyrrole $\mathbf{5 h}(21.0 \mathrm{mg}, 41.8 \mu \mathrm{mol})$ was dissolved in $\mathrm{CH}_{2} \mathrm{Cl}_{2}(2.0 \mathrm{~mL})$. NBS $(24.0 \mathrm{mg}$, $135 \mu \mathrm{mol}$ ) was added at $0{ }^{\circ} \mathrm{C}$ and the reaction mixture was stirred at ambient temperature overnight. To the reaction mixture was added silica gel and the solvent was evaporated in vacuo. Silica gel column chromatography (EtOAc: $n$-pentane $=1: 20)$ afforded compound 7 (25.1 mg, $38.0 \mu \mathrm{mol}, 91 \%$ ) as colorless solid.

${ }^{1} \mathbf{H}-N M R\left(300 \mathrm{MHz}, \mathrm{CDCl}_{3}\right): \delta=-0.44(\mathrm{~d}, J=8.4 \mathrm{~Hz}, 6 \mathrm{H}), 0.67(\mathrm{~s}, 9 \mathrm{H}), 2.37$ (s, 3H), 6.74 (s, 1H), $7.03(\mathrm{dd}, J=7.5,1.1 \mathrm{~Hz}, 1 \mathrm{H}), 7.14(\mathrm{dd}, J=7.6,1.6 \mathrm{~Hz}, 1 \mathrm{H}), 7.26-7.40(\mathrm{~m}, 4 \mathrm{H})$, 7.46-7.58 (m, 4H), $7.80(\mathrm{~d}, J=8.4 \mathrm{~Hz}, 2 \mathrm{H})$.

${ }^{13} \mathrm{C}-\mathrm{NMR}\left(75 \mathrm{MHz}, \mathrm{CDCl}_{3}\right) \delta=-4.2,-2.5,18.3,21.5,27.8,102.7,111.7,115.9,122.4$, $124.7,127.6,128.4,128.7,129.3,129.6,129.7,130.0,130.5,130.7,133.0,133.3,136.4$, 136.4, 140.3, 144.0.

IR (ATR): $\tilde{v}\left(\mathrm{~cm}^{-1}\right)=3257(\mathrm{~m}), 2956(\mathrm{w}), 2929(\mathrm{w}), 2893(\mathrm{w}), 2854(\mathrm{w}), 1493(\mathrm{~m}), 1387$ (m), $1333(\mathrm{~m}), 1162(\mathrm{~s}), 1089(\mathrm{~m})$.

M.p.: $220 \neg 222^{\circ} \mathrm{C}$.

ESI-HRMS: $\mathrm{C}_{29} \mathrm{H}_{32} \mathrm{Br}_{2} \mathrm{~N}_{2} \mathrm{O}_{2} \mathrm{SSi} / z$ calcd. $\left[\mathrm{M}+\mathrm{H}^{+}\right]$: 659.0393 found 659.0400 . 


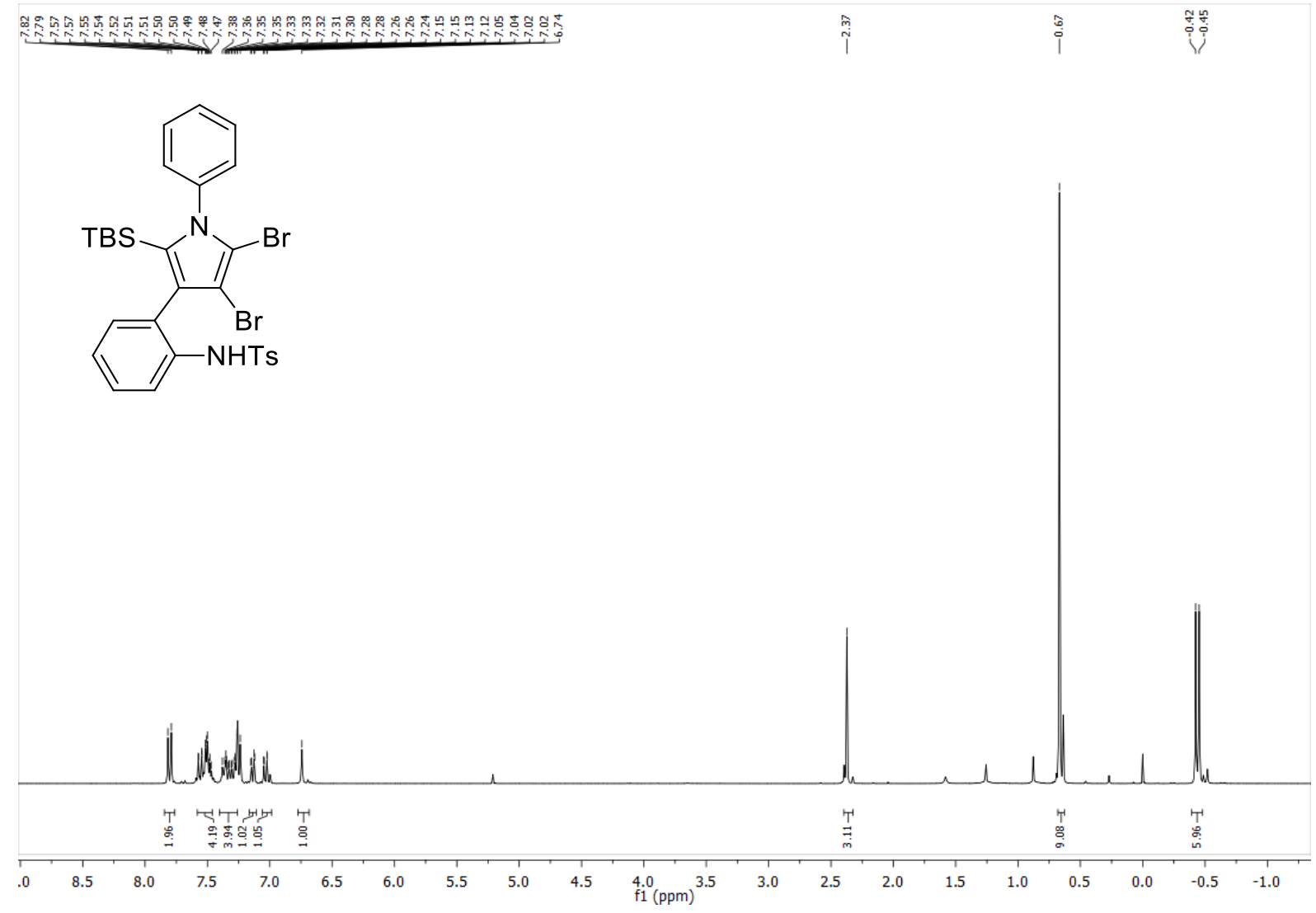

${ }^{1} \mathbf{H}-\mathbf{N M R}\left(300 \mathrm{MHz}, \mathrm{CDCl}_{3}\right)$

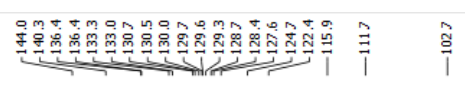
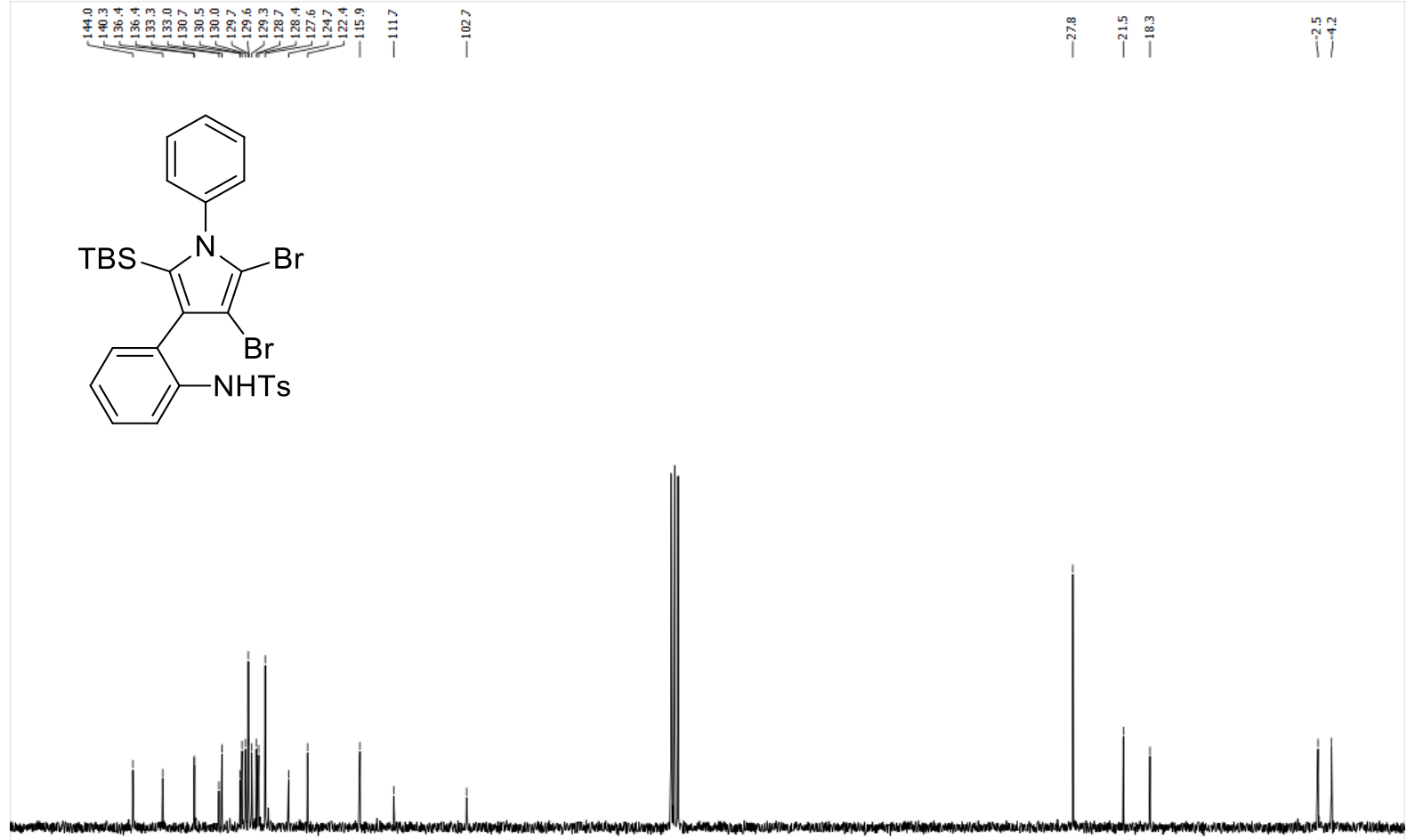

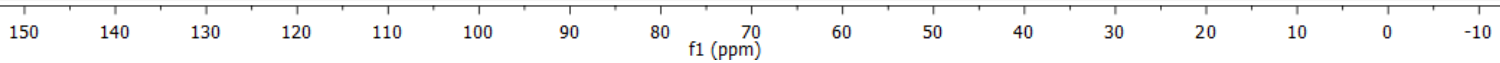

${ }^{13}$ C-NMR $\left(75 \mathrm{MHz}, \mathrm{CDCl}_{3}\right)$ 


\section{1-(tert-Butyldimethylsilyl)-2-phenyl-4-tosyl-2,4-dihydropyrrolo[3,4-b]indole (8)}<smiles>CC(C)(C)c1c2c(c(Br)n1-c1ccccc1)[N+](=S)c1ccccc1-2</smiles>

The bromide 7 (20 mg, $30.3 \mu \mathrm{mol})$ was diluted in DMF $(1.0 \mathrm{~mL})$. Afterwards triethylamine $(1.0 \mathrm{~mL}), \mathrm{PPh}_{3}(2.1 \mathrm{mg}, 8.0 \mu \mathrm{mol})$, and $\mathrm{PdCl}_{2}\left(\mathrm{PPh}_{3}\right)_{2}(5.6 \mathrm{mg}, 8.0 \mu \mathrm{mol})$ were added. The mixture was stirred $16 \mathrm{~h}$ at $70{ }^{\circ} \mathrm{C}$. After cooling to $\mathrm{rt}$, sat. $\mathrm{NH}_{4} \mathrm{Cl}$ solution was added and the mixture was extracted with EtOAc $(3 \times)$. The combined organic layers were dried over $\mathrm{Na}_{2} \mathrm{SO}_{4}$ and concentrated in vacuo. Silica gel column chromatography $($ EtOAc: $n$-pentane $=1: 20)$ afforded domino product $8(11.3 \mathrm{mg}, 19.5 \mu \mathrm{mol}, 64 \%)$ as yellow oil.

${ }^{1}$ H-NMR (400 MHz, $\left.\mathrm{CDCl}_{3}\right): \delta=-0.14(\mathrm{~s}, 6 \mathrm{H}), 0.71$ (s, 9H), 2.28 (s, 3H), 7.02-7.06 (m, 2H), 7.21-7.25 (m, 3H), 7.30-7.34 (m, 1H), 7.43-7.50 (m, 5H), 7.65-7.70 (m, 1H), 8.21$8.27(\mathrm{~m}, 1 \mathrm{H})$.

${ }^{13} \mathrm{C}-\mathrm{NMR}\left(101 \mathrm{MHz}, \mathrm{CDCl}_{3}\right) \delta=-3.9,19.7,21.5,27.0,93.1,118.1,121.7,123.6,124.5$, $125.3,126.5,127.3,128.4,128.6,128.9,129.4$, 130.4, 130.6, 133.0, 141.0, 144.1, 144.4 .

IR (ATR): $\tilde{v}\left(\mathrm{~cm}^{-1}\right)=2931(\mathrm{w}), 2890(\mathrm{w}), 2856(\mathrm{w}), 1597(\mathrm{w}), 1493(\mathrm{w}), 1461(\mathrm{w}), 1369$ (m), $1253(\mathrm{w}), 1176(\mathrm{~m}), 1090(\mathrm{w})$.

ESI-HRMS: $\mathrm{C}_{29} \mathrm{H}_{31} \mathrm{BrN}_{2} \mathrm{O}_{2} \mathrm{SSi} \mathrm{m} / z$ calcd. $\left[\mathrm{M}+\mathrm{H}^{+}\right]: 579.1132$ found 579.1135 . 


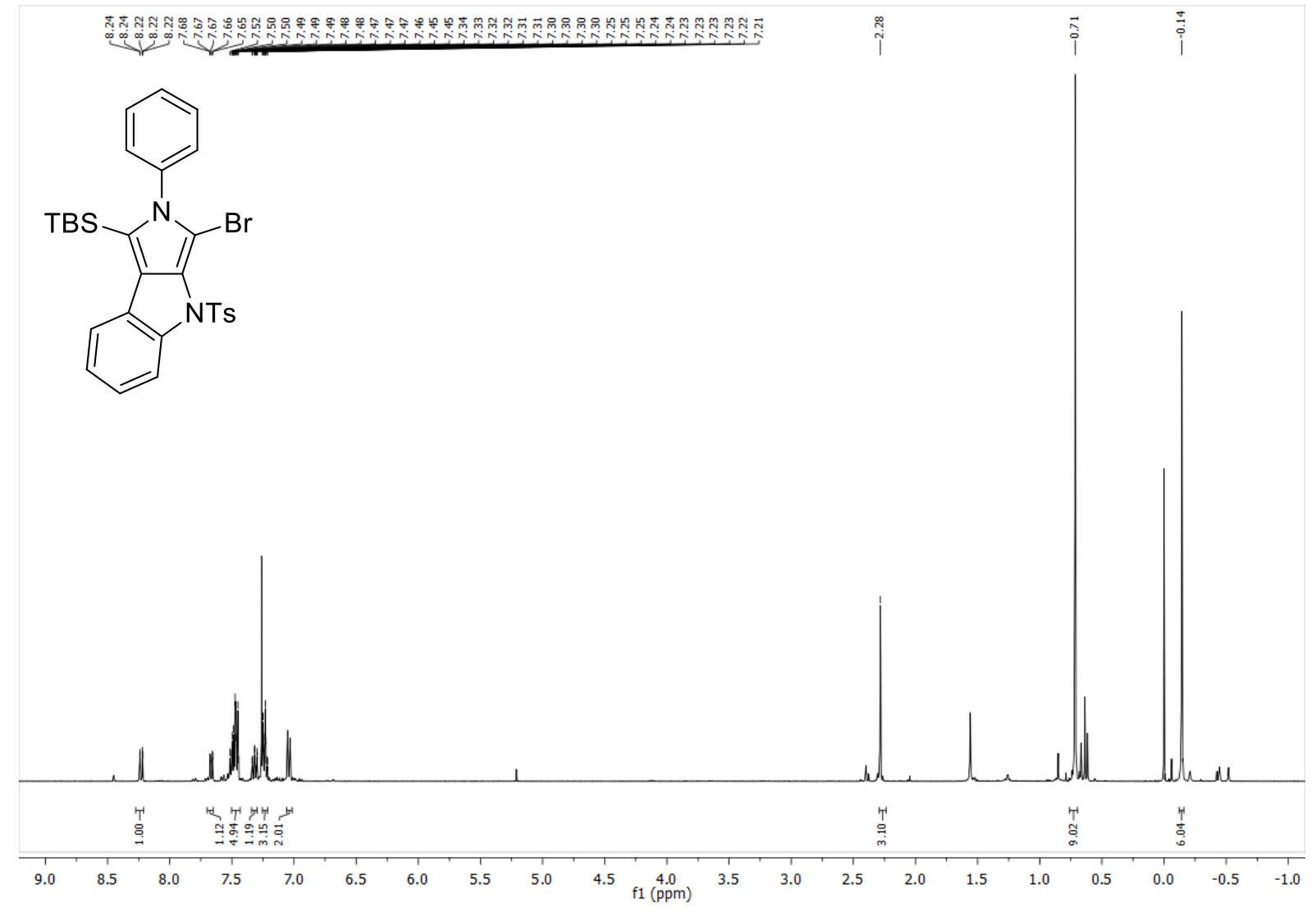

${ }^{\mathbf{1}} \mathbf{H}-\mathbf{N M R}\left(400 \mathrm{MHz}, \mathrm{CDCl}_{3}\right)$

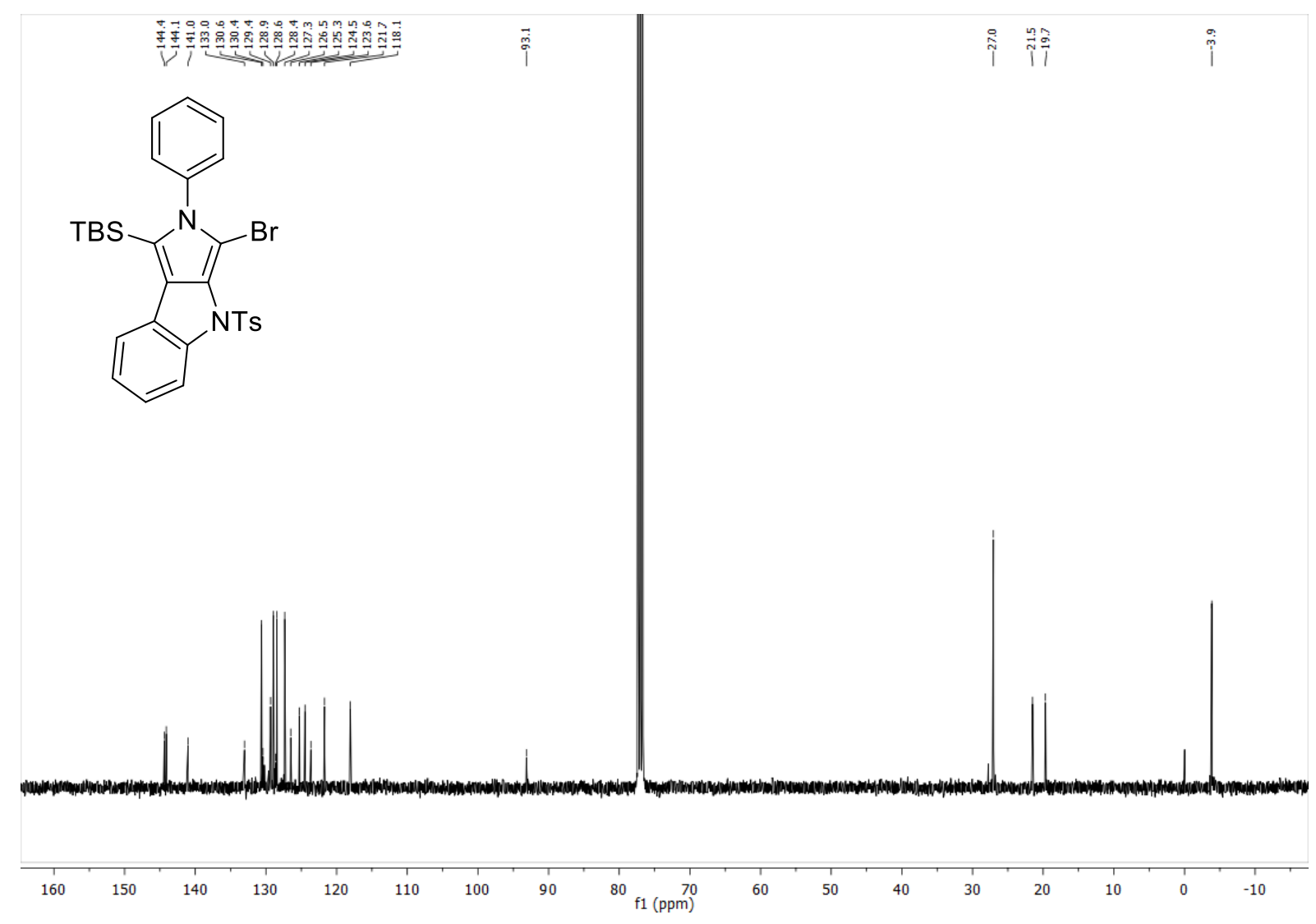

${ }^{13}$ C-NMR (101 MHz, $\left.\mathrm{CDCl}_{3}\right)$ 


\section{$N$-(2-(5-Bromo-2-(tert-butyldimethylsilyl)-1-phenyl-1H-pyrrol-3-yl)phenyl)-4-}

methylbenzenesulfonamide (9)<smiles>CC(c1cc(Br)n(-c2ccccc2)c1-c1ccccc1N)C(F)(F)F</smiles>

The pyrrole $5 \mathbf{h}(24.7 \mathrm{mg}, 49.1 \mu \mathrm{mol})$ was dissolved in $\mathrm{CH}_{2} \mathrm{Cl}_{2}(2.0 \mathrm{~mL})$. NBS $(9.5 \mathrm{mg}$, $53.3 \mu \mathrm{mol}$ ) was added at $0{ }^{\circ} \mathrm{C}$ and the reaction mixture was stirred at ambient temperature overnight. To the reaction mixture was added silica gel and the solvent was evaporated in vасио. Silica gel column chromatography (EtOAc: $n$-pentane $=1: 20)$ afforded compound 8 (23.6 mg, $40.6 \mu \mathrm{mol}, 83 \%$ ) as colorless solid.

${ }^{1}$ H-NMR (300 MHz, $\mathrm{CDCl}_{3}$ ): $\delta=-0.52$ (s, 3H), -0.44 (s, 3H), 0.64 (s, 9H), 2.40 (s, 3H), 5.22 (s, 1H), 6.69 (s, 1H), $7.05(\mathrm{td}, J=7.4,1.2 \mathrm{~Hz}, 1 \mathrm{H}), 7.16(\mathrm{dd}, J=7.6,1.6 \mathrm{~Hz}, 1 \mathrm{H}), 7.21-7.25$ $(\mathrm{m}, 1 \mathrm{H}), 7.26-7.33(\mathrm{~m}, 3 \mathrm{H}), 7.42-7.53(\mathrm{~m}, 3 \mathrm{H}), 7.55-7.63(\mathrm{~m}, 2 \mathrm{H}), 7.69$ (dd, $J=8.2,1.2 \mathrm{~Hz}$, $1 \mathrm{H})$.

${ }^{13}$ C-NMR $\left(76 \mathrm{MHz}, \mathrm{CDCl}_{3}\right) \delta=-4.2,-2.6,18.2,21.6,27.8,109.8,113.8,119.4,123.4$, 127.2 , 128.3, 128.5, 128.6, 128.9, 129.3, 129.6, 130.1, 130.6, 131.9, 132.0, 132.1, 135.7, 136.2, 140.1, 143.9.

IR (ATR): $\tilde{v}\left(\mathrm{~cm}^{-1}\right)=3298(\mathrm{w}), 2932$ (w), $2891(\mathrm{w}), 2855$ (w), 1490 (w), 1379 (m), 1332 (m), 1163 (s), $1091(\mathrm{~m})$.

M.p.: $190 \neg 192{ }^{\circ} \mathrm{C}$.

ESI-HRMS: $\mathrm{C}_{29} \mathrm{H}_{33} \mathrm{BrN}_{2} \mathrm{O}_{2} \mathrm{SSi} / z$ calcd. $\left[\mathrm{M}+\mathrm{H}^{+}\right]$: 581.1288 found 581.1294. 


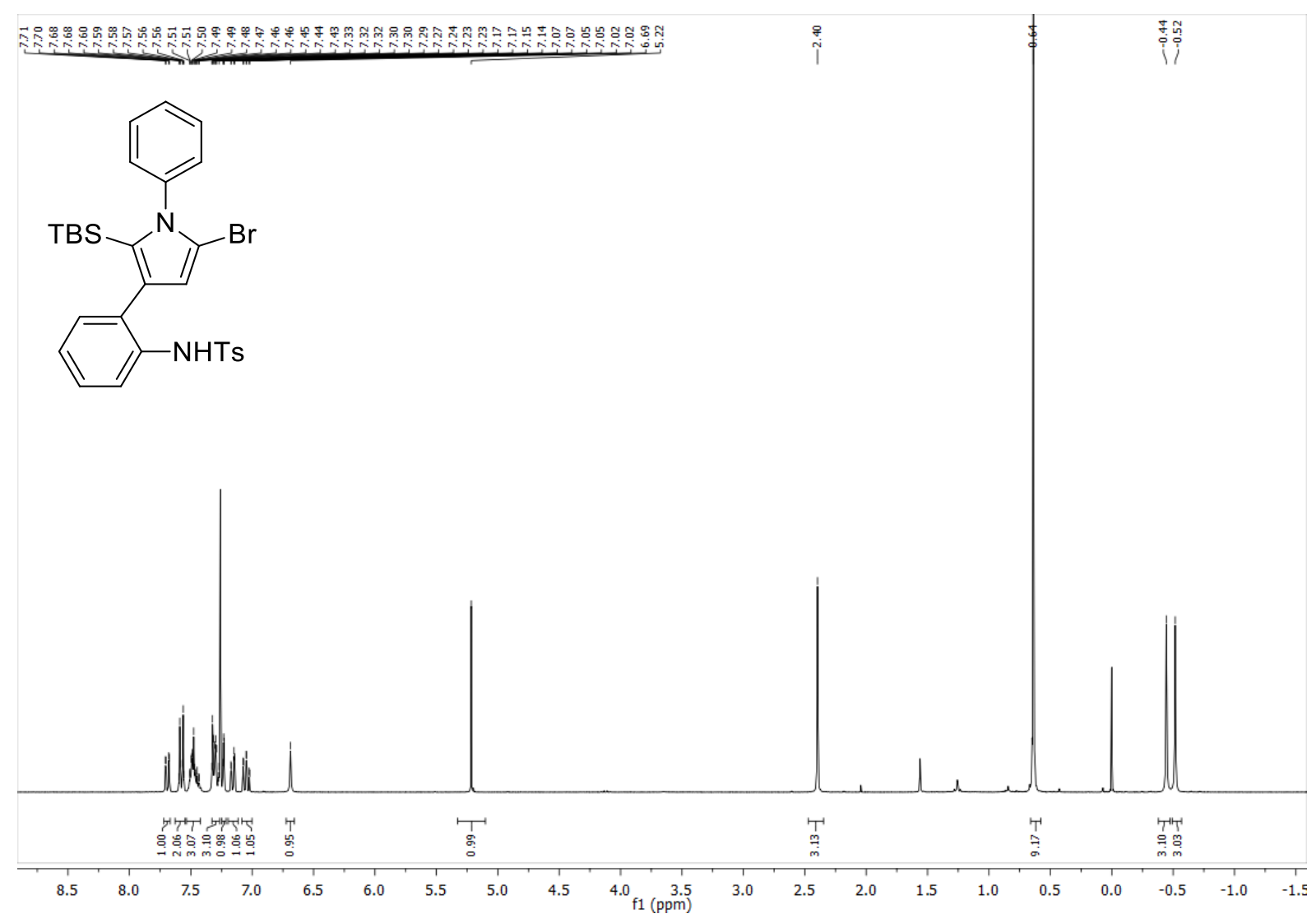

${ }^{1} \mathbf{H}-\mathbf{N M R}\left(300 \mathrm{MHz}, \mathrm{CDCl}_{3}\right)$

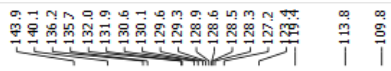<smiles>CC(C)(C)C(F)(F)c1cc(-c2ccccc2NC(F)(F)F)c(Br)n1-c1ccccc1</smiles>
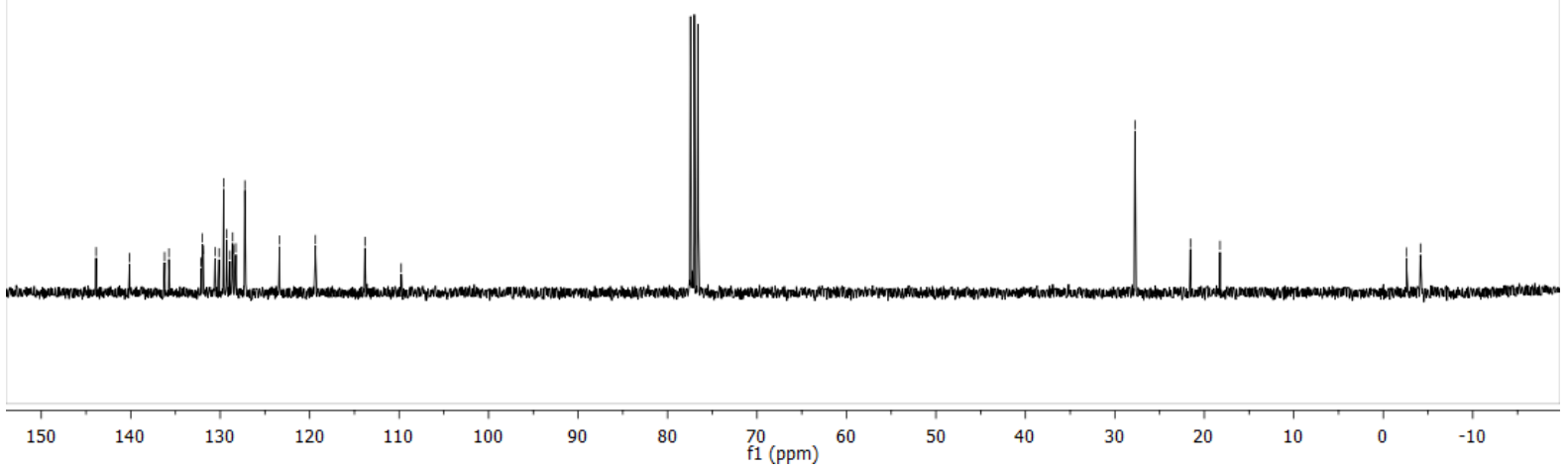

${ }^{13} \mathbf{C}$-NMR (76 MHz, $\mathrm{CDCl}_{3}$ ) 
4-Methyl- $N$-(2-(1-phenyl-1H-pyrrol-3-yl)phenyl)benzenesulfonamide (5g)

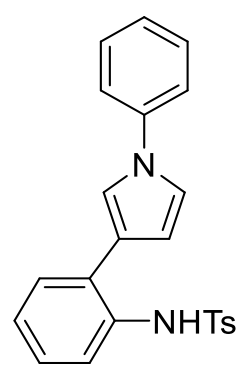

The pyrrole $\mathbf{5 h}(24.4 \mathrm{mg}, 48.5 \mu \mathrm{mol})$ was dissolved in $\mathrm{CH}_{2} \mathrm{Cl}_{2}(5.0 \mathrm{~mL})$. At $0{ }^{\circ} \mathrm{C}$, acetyl chloride ( 1 drop) and aluminium trichloride $(8.0 \mathrm{mg}, 60.0 \mu \mathrm{mol})$ were added and the reaction mixture was stirred at ambient temperature for $1 \mathrm{~h}$. To the reaction mixture was added water and the aqueous layer was extracted with EtOAc $(3 \times)$. The combined organic layers were dried over $\mathrm{Na}_{2} \mathrm{SO}_{4}$ and concentrated in vacuo. Silica gel column chromatography (EtOAc: $n$-pentane $=1: 10)$ afforded compound $\mathbf{5 g}(15.7 \mathrm{mg}, 36.5 \mu \mathrm{mol}, 75 \%)$ as colorless solid.

For analytical data see page S80. 


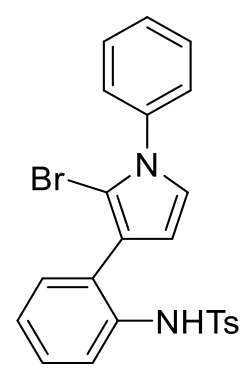

The pyrrole $5 \mathrm{~g}(13.0 \mathrm{mg}, 33.5 \mu \mathrm{mol})$ was dissolved in $\mathrm{CH}_{2} \mathrm{Cl}_{2}(1.0 \mathrm{~mL})$. At $0{ }^{\circ} \mathrm{C}$, NBS $(6.2 \mathrm{mg}, 34.8 \mu \mathrm{mol})$ was added and the reaction mixture was stirred at $0{ }^{\circ} \mathrm{C}$ for $1 \mathrm{~h}$. To the reaction mixture was added silica gel and the solvent was evaporated in vacuo. Silica gel column chromatography (EtOAc: $n$-pentane $=1: 10)$ afforded compound $\mathbf{1 0}$ (9.3 mg, $19.9 \mu \mathrm{mol}, 59 \%)$ as colorless oil.

${ }^{1}$ H-NMR (400 MHz, $\left.\mathrm{CDCl}_{3}\right): \delta=2.35(\mathrm{~s}, 3 \mathrm{H}), 5.96(\mathrm{~d}, J=3.1 \mathrm{~Hz}, 1 \mathrm{H}), 6.95$ (s, 1H), 6.99 (d, $J=3.1 \mathrm{~Hz}, 1 \mathrm{H}), 7.11(\mathrm{td}, J=7.5,1.2 \mathrm{~Hz}, 1 \mathrm{H}), 7.20(\mathrm{td}, J=7.7,1.8 \mathrm{~Hz}, 3 \mathrm{H}), 7.27-7.32(\mathrm{~m}$, 1H), 7.39-7.53 (m, 5H), 7.58-7.64 (m, 2H), 7.68 (dd, $J=8.3,1.3 \mathrm{~Hz}, 1 \mathrm{H})$.

${ }^{13}$ C-NMR $\left(101 \mathrm{MHz}, \mathrm{CDCl}_{3}\right) \delta=21.5,102.5,110.7,120.0,120.6,124.3,124.4,126.2$, $126.5,127.2,128.2,128.6,129.1,129.6,131.5,134.9,136.4,139.1,143.7$.

IR (ATR): $\tilde{v}\left(\mathrm{~cm}^{-1}\right)=3331(\mathrm{w}), 2922(\mathrm{w}), 2857(\mathrm{w}), 1594(\mathrm{w}), 1497(\mathrm{~m}), 1398(\mathrm{~m}), 1334$ (m), $1160(\mathrm{~s}), 1088(\mathrm{~m})$.

ESI-HRMS: $\mathrm{C}_{23} \mathrm{H}_{19} \mathrm{BrN}_{2} \mathrm{O}_{2} \mathrm{~S} m / z$ calcd. $\left[\mathrm{M}+\mathrm{Na}^{+}\right]$: 489.0243 Found 489.0244 . 
<smiles>FC(F)(S)Nc1ccccc1Br</smiles>

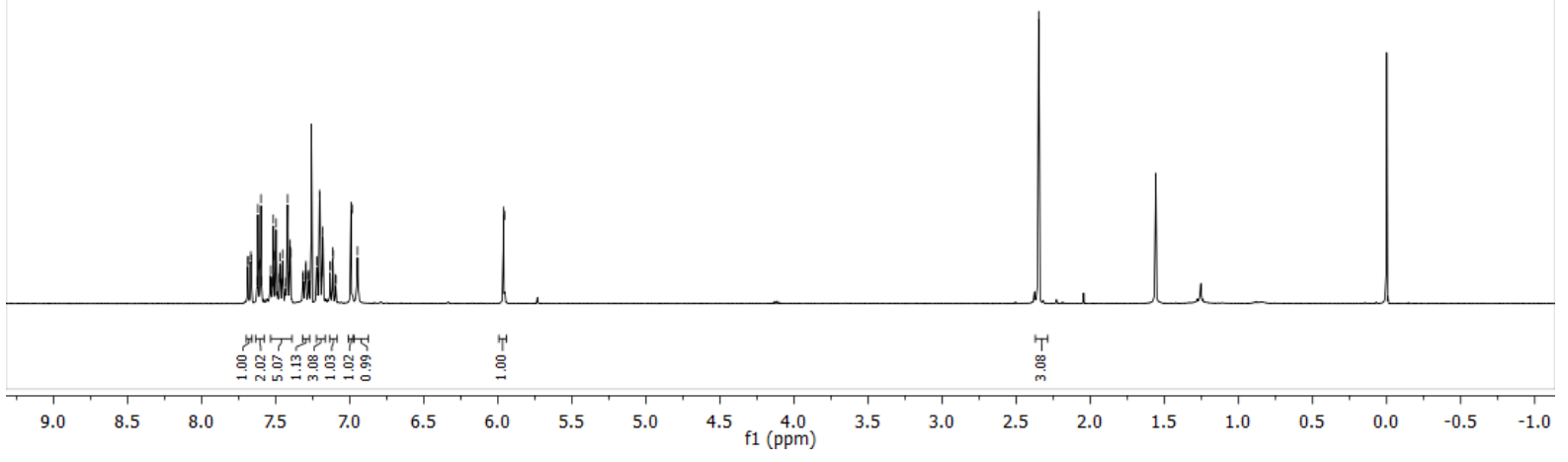

${ }^{1} \mathbf{H}-\mathbf{N M R}\left(400 \mathrm{MHz}, \mathrm{CDCl}_{3}\right)$

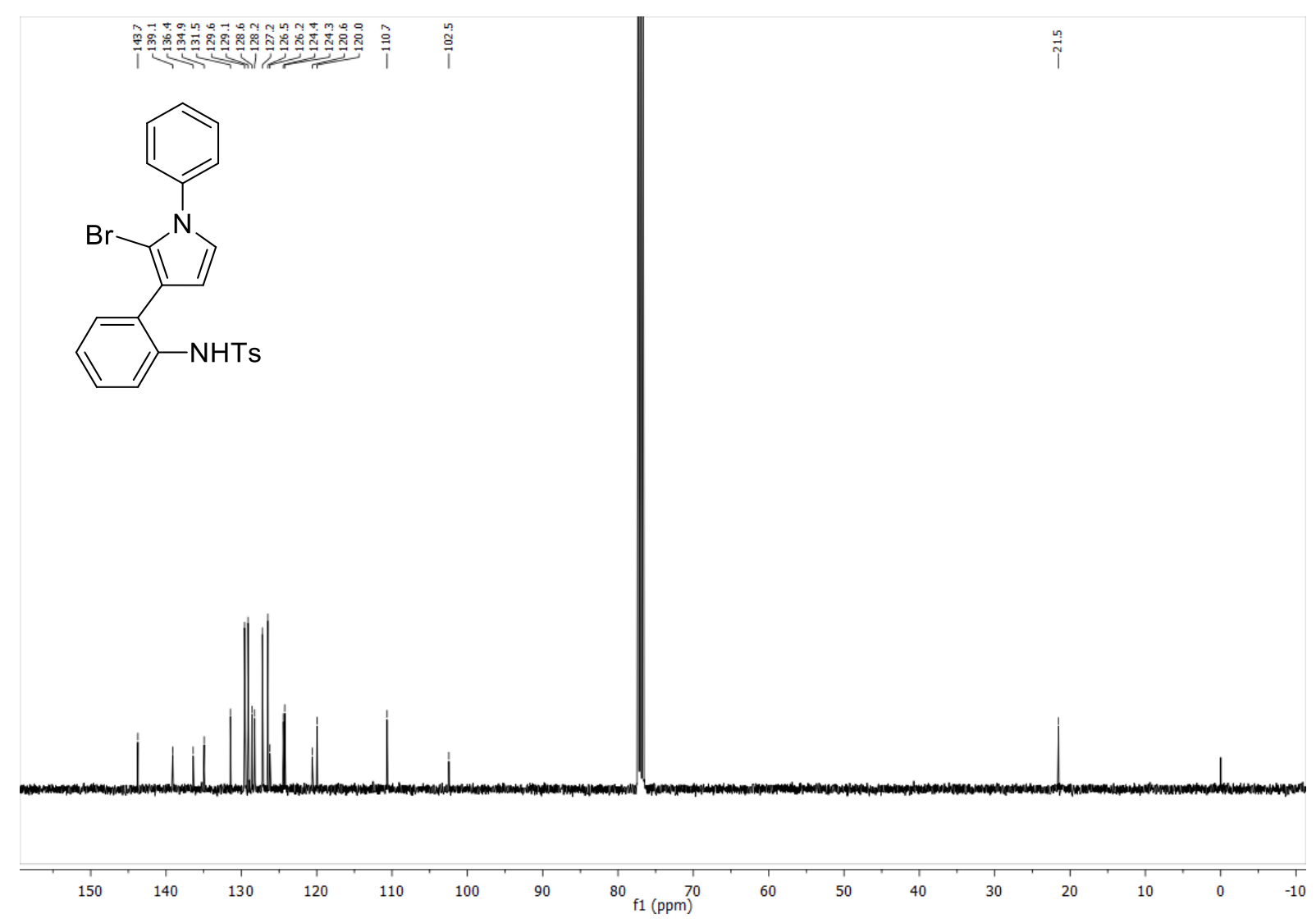

${ }^{13}$ C-NMR (101 MHz, $\left.\mathrm{CDCl}_{3}\right)$ 


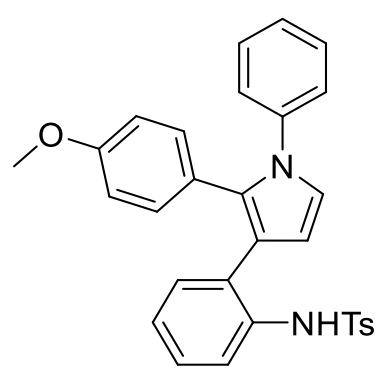

The pyrrole $\mathbf{5 h}(24.9 \mathrm{mg}, 49.5 \mu \mathrm{mol})$ was dissolved in $\mathrm{CH}_{2} \mathrm{Cl}_{2}(2.0 \mathrm{~mL})$. At $0{ }^{\circ} \mathrm{C}, \mathrm{BCl}_{3}(1 \mathrm{M}$ in hexane, $60 \mu \mathrm{L}, 60.0 \mu \mathrm{mol}$ ) was added and the reaction mixture was stirred 30 minutes at $0^{\circ} \mathrm{C}$. Afterwards the solvent was evaporated in vacuo. After drying under high vacuum for 30 minutes, the residue was dissolved in DME (2.0 mL). 4-Iodoanisole $(37.7 \mathrm{mg}, 161 \mu \mathrm{mol})$, $\mathrm{Pd}\left(\mathrm{PPh}_{3}\right)_{4}(8.0 \mathrm{mg}, 6.92 \mu \mathrm{mol}, 14 \mathrm{~mol}-\%)$, and $\mathrm{Na}_{2} \mathrm{CO}_{3}$-solution $(2 \mathrm{M}, 500 \mu \mathrm{L})$ were added. The reaction mixture was refluxed for $4 \mathrm{~h}$. After cooling to $\mathrm{rt}$, sat. $\mathrm{NH}_{4} \mathrm{Cl}$ solution was added. The aqueous layer was extracted with EtOAc $(3 \times)$. The combined organic layers were dried over $\mathrm{Na}_{2} \mathrm{SO}_{4}$ and concentrated in vauco. Silica gel column chromatography (EtOAc: $n$-pentane = 1:10) afforded compound 11 (7.0 mg, $14.2 \mu \mathrm{mol}, 29 \%$ over two steps) as yellow oil.

${ }^{1} \mathbf{H}-\mathrm{NMR}\left(500 \mathrm{MHz}, \mathrm{CDCl}_{3}\right): \delta=2.27$ (s, 3H), 3.80 (s, 3H), $5.99(\mathrm{~d}, J=2.1 \mathrm{~Hz}, 1 \mathrm{H}), 6.46$ (d, $J=1.9 \mathrm{~Hz}, 1 \mathrm{H}), 6.79$ (d, $J=8.8 \mathrm{~Hz}, 2 \mathrm{H}), 7.00-7.06(\mathrm{~m}, 2 \mathrm{H}), 7.08-7.15(\mathrm{~m}, 6 \mathrm{H}), 7.26-7.28$ (m, 2H), 7.30-7.33 (m, 1H), 7.34-7.38 (m, 2H), 7.54-7.62 (m, 2H), $7.68(\mathrm{dd}, J=8.7,1.3 \mathrm{~Hz}$, $1 \mathrm{H})$.

${ }^{13}$ C-NMR $\left(126 \mathrm{MHz}, \mathrm{CDCl}_{3}\right) \delta=21.4,55.2,109.9,113.7,120.4,121.2,121.9,124.7,124.8$, $125.6,127.0,127.1,127.8,127.9$, 129.1, 129.6, 129.6, 130.3, 134.3, 134.8, 136.5, 139.9, $143.7,158.6$.

IR (ATR): $\tilde{v}\left(\mathrm{~cm}^{-1}\right)=3323(\mathrm{w}), 2957(\mathrm{w}), 2922(\mathrm{w}), 2836(\mathrm{w}), 1597(\mathrm{~m}), 1493(\mathrm{~s}), 1336(\mathrm{~m})$, $1248(\mathrm{~m}), 1165$ (s), 1092 (m).

ESI-HRMS: $\mathrm{C}_{30} \mathrm{H}_{26} \mathrm{~N}_{2} \mathrm{O}_{3} \mathrm{~S} m / z$ calcd. $\left[\mathrm{M}+\mathrm{H}^{+}\right]$: 495.1737 found 495.1738 . 


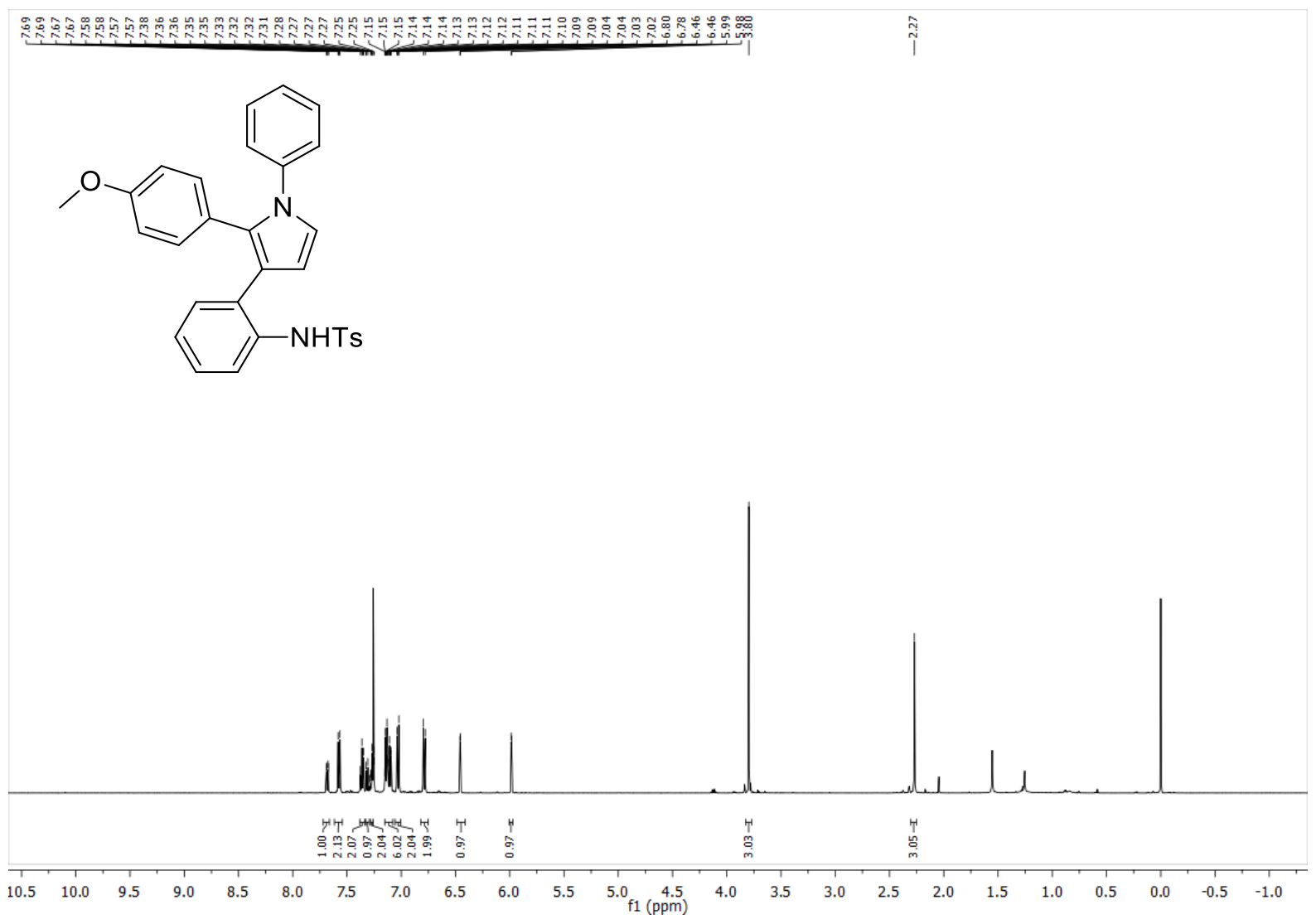

${ }^{1} \mathbf{H}-\mathbf{N M R}\left(500 \mathrm{MHz}, \mathrm{CDCl}_{3}\right)$

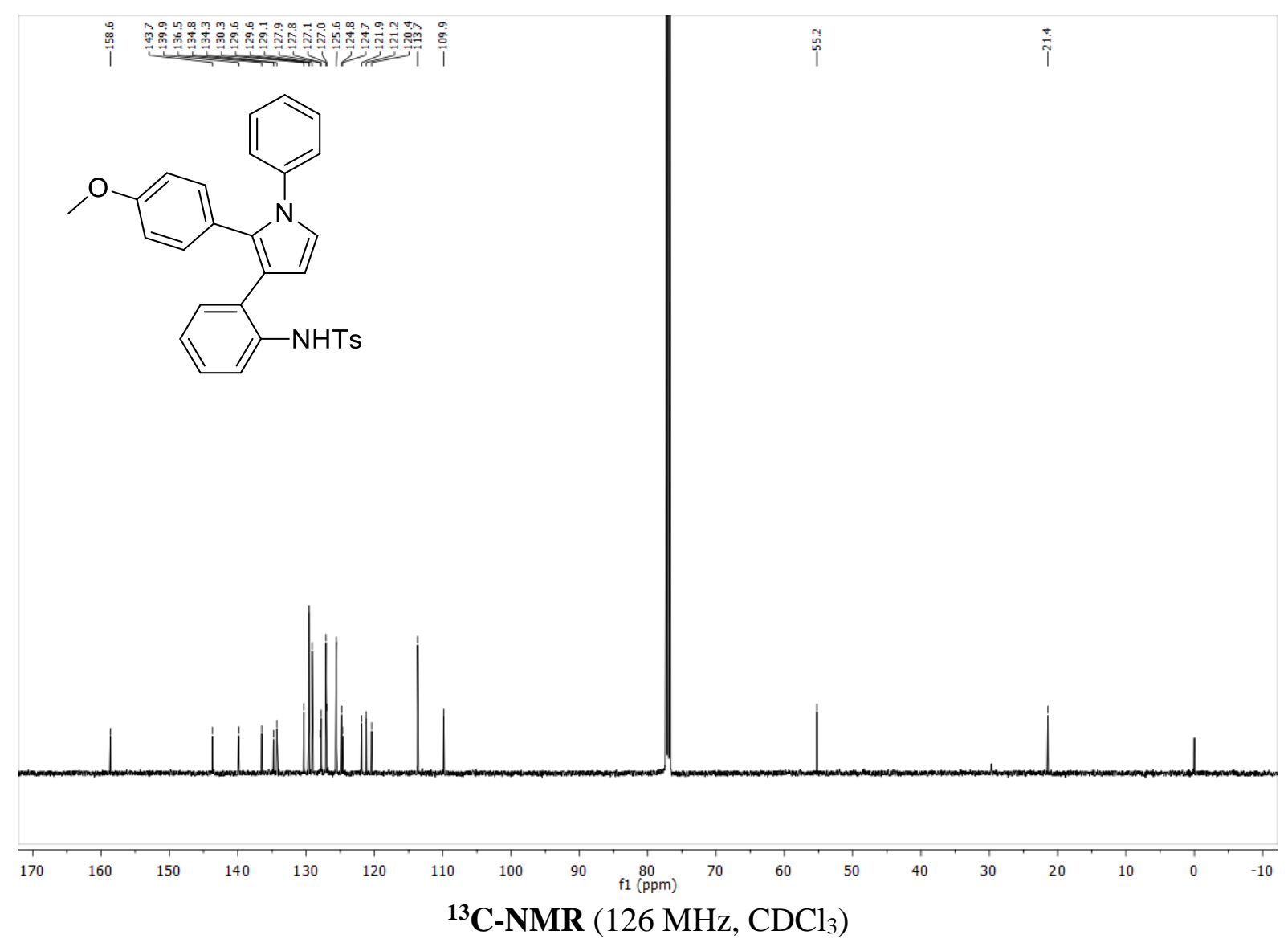




\section{9) Crystal Structure Determination of Compound 3f}

A pale brown tablet-shaped crystal was mounted in inert oil on a glass fibre and transferred to the cold gas stream of an Oxford "Xcalibur E" diffractometer. Mo Ka radiation was employed for the intensity measurements. An absorption correction was implemented on the basis of multi-scans. The structure was refined anisotropically on $F^{2}$ using the program SHELXL-2017. ${ }^{5}$ Hydrogen atoms were included using rigid methyl groups or a riding model starting from calculated positions.

Crystallographic data are summarized in Table S1. Additionally, complete data have been deposited with the Cambridge Crystallographic Data Centre under the number CCDC 1958050. Copies of the data can be obtained free of charge from www.ccdc.cam.ac.uk/data_request/cif.

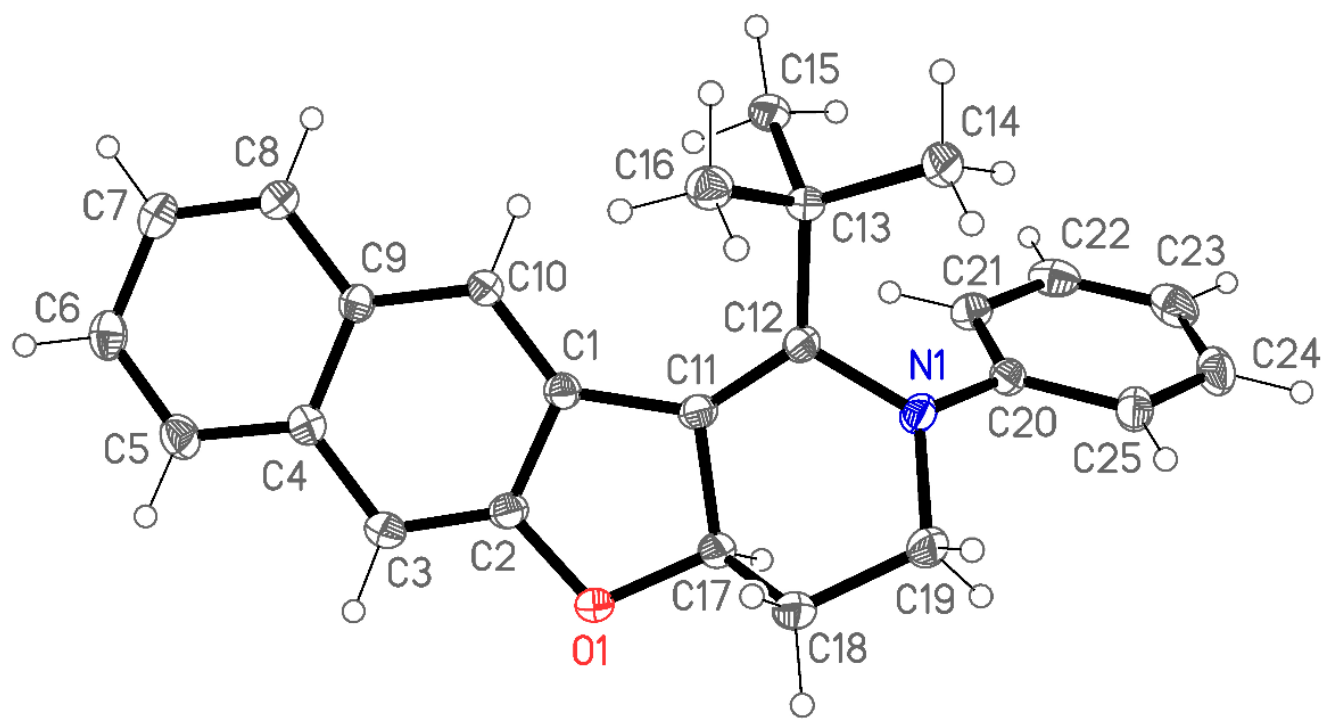

Figure S1. The structure of compound $\mathbf{3 f}$ in the crystal. Ellipsoids represent 50\% probability levels. 
Table S1: Crystallographic data and structure refinement details.

\begin{tabular}{|c|c|}
\hline Compound & $3 f$ \\
\hline Formula & $\mathrm{C}_{25} \mathrm{H}_{25} \mathrm{NO}$ \\
\hline$M_{\mathrm{r}}$ & 355.46 \\
\hline Cryst. size (mm) & $0.45 \times 0.35 \times 0.2$ \\
\hline Crystal system & triclinic \\
\hline Space group & $P(-1)$ \\
\hline Temperature $\left({ }^{\circ} \mathrm{C}\right)$ & -163 \\
\hline$a(\AA)$ & $8.3325(5)$ \\
\hline$b(\AA)$ & $9.7944(5)$ \\
\hline$c(\AA)$ & $12.6679(5)$ \\
\hline$\alpha\left(^{\circ}\right)$ & $68.278(5)$ \\
\hline$\beta\left(^{\circ}\right)$ & $78.444(5)$ \\
\hline$\gamma\left({ }^{\circ}\right)$ & $86.722(5)$ \\
\hline$V\left(\AA^{3}\right)$ & 940.80 \\
\hline Z & 2 \\
\hline$D_{\mathrm{x}}\left(\mathrm{Mg} \mathrm{m}^{-3}\right)$ & 1.255 \\
\hline$\lambda(\AA)$ & 0.71073 \\
\hline$\mu\left(\mathrm{mm}^{-1}\right)$ & 0.08 \\
\hline Transmissions & $0.965-1.000$ \\
\hline$F(000)$ & 380 \\
\hline $2 \theta_{\max }\left(^{\circ}\right)$ & 60 \\
\hline Refl. measured & 47793 \\
\hline Refl. indep. & 5408 \\
\hline$R_{\text {int }}$ & 0.035 \\
\hline Parameters & 247 \\
\hline$w R\left(F^{2}\right.$, all refl. $)$ & 0.123 \\
\hline$R(F,>4 \sigma(F))$ & 0.046 \\
\hline$S$ & 1.03 \\
\hline $\operatorname{Max} . \Delta p\left(\mathrm{e} \AA^{-3}\right)$ & $0.40 /-0.23$ \\
\hline
\end{tabular}




\section{0) References}

[1] Schitter, T.; Jones, P. G.; Werz, D. B. Chem. Eur. J. 2018, 24, 13446.

[2] The alcohol 14 was prepared by using a previously reported protocol (Schitter, T.; Jones, P. G.; Werz, D. B. Chem. Eur. J. 2018, 24, 13446.)

[3] Schitter, T.; Roy, N. J.; Jones, P. G.; Werz, D. B. Org. Lett. 2019, 21, 640.

[4] The alcohol 24 was prepared by using a previously reported protocol (Schitter, T.; Roy, N. J.; Jones, P. G.; Werz, D. B. Org. Lett. 2019, 21, 640.)

[5] Sheldrick, G. M. Acta Cryst. 2015, C71, 3. 